\title{
Large-Eddy Simulation of the Evolving Stable Boundary Layer over Flat Terrain
}

\author{
R.T. Cederwall
}

Ph. D. Dissertation

Stanford University

January 1, 2002

U.S. Department of Energy

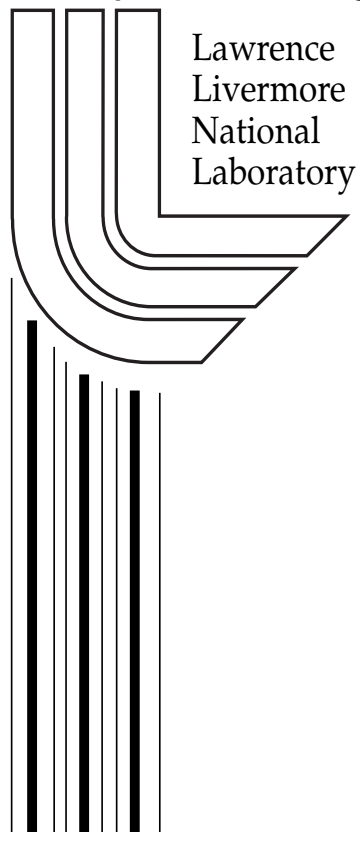




\section{DISCLAIMER}

This document was prepared as an account of work sponsored by an agency of the United States Government. Neither the United States Government nor the University of California nor any of their employees, makes any warranty, express or implied, or assumes any legal liability or responsibility for the accuracy, completeness, or usefulness of any information, apparatus, product, or process disclosed, or represents that its use would not infringe privately owned rights. Reference herein to any specific commercial product, process, or service by trade name, trademark, manufacturer, or otherwise, does not necessarily constitute or imply its endorsement, recommendation, or favoring by the United States Government or the University of California. The views and opinions of authors expressed herein do not necessarily state or reflect those of the United States Government or the University of California, and shall not be used for advertising or product endorsement purposes.

This work was performed under the auspices of the U. S. Department of Energy by the University of California, Lawrence Livermore National Laboratory under Contract No. W-7405-Eng-48.

This report has been reproduced directly from the best available copy.

Available to DOE and DOE contractors from the

Office of Scientific and Technical Information

P.O. Box 62, Oak Ridge, TN 37831

Prices available from (423) 576-8401

http:/ /apollo.osti.gov/bridge/

Available to the public from the National Technical Information Service

U.S. Department of Commerce 5285 Port Royal Rd., Springfield, VA 22161

http://www.ntis.gov/

OR

Lawrence Livermore National Laboratory

Technical Information Department's Digital Library

http://www.llnl.gov/tid/Library.html 


\title{
LARGE-EDDY SIMULATION OF THE EVOLVING STABLE BOUNDARY LAYER OVER FLAT TERRAIN
}

\author{
A DISSERTATION \\ SUBMitTED TO THE COMMITTEE ON GRADUATE STUdIES \\ OF STANFORD UNIVERSITY \\ IN PARTIAL FULFILLMENT OF THE REQUIREMENTS \\ FOR THE DEGREE OF \\ DOCTOR OF PHILOSOPHY \\ IN \\ ATMOSPHERIC SCIENCES
}

Richard Townsend Cederwall

December 2001 
(C) Copyright 2002 by Richard Townsend Cederwall All Rights Reserved 
I certify that I have read this dissertation and that in my opinion it is fully adequate, in scope and in quality, as a dissertation for the degree of Doctor of Philosophy.

$$
\begin{gathered}
\text { Robert L. Street } \\
\text { (Principal Adviser) }
\end{gathered}
$$

I certify that I have read this dissertation and that in my opinion it is fully adequate, in scope and in quality, as a dissertation for the degree of Doctor of Philosophy.

\section{Joel H. Ferziger}

I certify that I have read this dissertation and that in my opinion it is fully adequate, in scope and in quality, as a dissertation for the degree of Doctor of Philosophy.

\section{Brian J. Cantwell}

I certify that I have read this dissertation and that in my opinion it is fully adequate, in scope and in quality, as a dissertation for the degree of Doctor of Philosophy.

$$
\text { Jeffrey R. Koseff }
$$

Approved for the University Committee on Graduate Studies: 


\section{Abstract}

The stable boundary layer (SBL) in the atmosphere is of considerable interest because it is often the 'worst case' scenario for air pollution studies and health effect assessments associated with the accidental release of toxic material. Traditional modeling approaches used in such studies do not simulate the non-steady character of the velocity field, and hence often overpredict concentrations while underpredicting spatial coverage of potentially harmful concentrations of airborne material. The challenge for LES is to be able to resolve the rather small energy-containing eddies of the SBL while still maintaining an adequate domain size. This requires that the subgrid-scale (SGS) parameterization of turbulence incorporate an adequate representation of turbulent energy transfer. Recent studies have shown that both upscale and downscale energy transfer can occur simultaneously, but that overall the net transfer is downscale. Including the upscale transfer of turbulent energy (energy backscatter) is particularly important near the ground and under stably-stratified conditions.

The goal of this research is to improve our ability to realistically simulate the SBL. The large-eddy simulation (LES) approach with its subgrid-scale (SGS) turbulence model does a better job of capturing the temporally and spatially varying features of the SBL than do Reynolds-averaging models. The scientific objectives of this research are:

1. to characterize features of the evolving SBL structure for a range of meteorological conditions (wind speed and surface cooling),

2. to simulate realistically the transfer of energy between resolved and subgrid scales, and

3. to apply results to improve simulation of dispersion in the SBL.

An LES framework is used that is accurate numerically to second order. The SGS turbulence model is a dynamic, mixed model. Simulations are conducted over a range of forcing conditions specified in terms of the geostrophic wind and the surface cooling. The environment is simplified by being cloud-free and over flat terrain. 
The several SBLs simulated illustrate the key role of mechanical turbulence supported by the geostrophic forcing, and the lesser competing effects of turbulence damping by buoyancy that develops in response to the surface cooling. With sufficient geostrophic forcing, the SBL has continuous turbulence with periods of enhanced turbulence. As the surface cooling is increased and/or the geostrophic forcing decreased, the periods of enhanced turbulence become less prevalent and the overall intensity of continuous turbulence is reduced. At some point of increased surface cooling and/or reduced geostrophic forcing, the turbulence can no longer be maintained. A forcing stability index that represents effects of surface buoyancy flux $\left(B_{0}\right)$ and geostrophic wind $(\mathrm{G})$ is defined as $F_{s}=B_{0} / G^{2}|f|$. The bulk stability (characterized by the bulk Richardson number $R_{B}$ ) of the SBL that evolves under specific $B_{0}$ and $G$ forcing pairs is found to be described well by the relation $R_{B}=8 / 3 F_{s}$.

The SGS model used allows for backscatter (upscale transfer) of turbulent kinetic energy (TKE) and thermal energy. The TKE backscatter is dominated by the interaction of the streamwise velocity component with the wall-normal shear stress. The thermal backscatter occurs during ejections of cool surface air, associated with the action of coherent structures in the flow. Results from applying a vortex identification method highlight organized activity in the SBL, especially near the ground. The strong SBL exhibits a two-regime structure in the vertical direction, with vorticity dominating the lower part and larger-scale wave-like motions dominating the upper part and continuing above the SBL. Simulation of episodes of enhanced turbulence are made possible by inclusion of energy backscatter. The episodes are associated with the breakdown of large-scale wave-like activity.

The implications for dispersion in the SBL are demonstrated by releasing marker particles in LES-generated wind fields for an SBL with an enhanced turbulence event. Comparisons are made between simulated plumes before and during the event. The effect of the enhanced turbulence is to spread the plume over a larger volume. This is caused by two mechanisms: (1) mixing due to the increased small scale turbulence and (2) differential advection after the mixing begins due to the presence of a strong vertical gradient in horizontal wind direction. Comparisons are also made with simulated plumes from particles released into a convective boundary layer. The presence of large convective eddies supports strong mixing in the vertical direction. 
There is little additional horizontal spread due to differential advection since the mean horizontal wind is nearly constant with height in the well-mixed convective boundary layer. Eddy diffusivities are estimated directly from LES fields. These values are compared with estimates from algorithms used in practical dispersion models. Agreement is surprisingly good for the undisturbed SBL; however, the practical models cannot capture effects of enhanced turbulence on eddy diffusion during episodes. 


\section{Acknowledgments}

First, I would like to express my appreciation for the guidance, support, and especially the patience of my advisor, Bob Street, over the 20 years of our academic adventure together. He has been a great mentor on how to balance all the demands of professional life, and his cheerful 'Onward' kept me focused on the goal. I also thank the members of my committee: Joel Ferziger, Brian Cantwell, and Jeff Koseff, as well as Bob Bornstein and Steve Klotz who were early members of the committee; thanks go especially to Bob Bornstein, my MS thesis advisor at SJSU, who encouraged me to pursue my PhD studies at Stanford.

Early parts of the research were supported by the U.S. Army, under the technical guidance of Ron Meyers. Bill Ohmstede worked with me on the early LES model development - his wisdom and meteorological insight were most valuable. LLNL management, especially Lynn Anspaugh, Marv Dickerson, Tom Sullivan, Allen Grossman, Bill Dannevik, Don Ermak, and Gayle Sugiyama, have been supportive of this longterm effort; the LLNL Education Program has provided continual financial coverage of Stanford fees. Many LLNL colleagues have been cheerleaders over the years. In particular, Joe Shinn gave me the big perspective when it counted, Marty Leach provided gentle nudges and good ideas along the way, and Ron Calhoun (also a PhD student under Bob Street) gave me lots of coaching, helped me become Tex-fluent, and set up a productive computer environment for me for the final push (before he dashed off from LLNL to his new faculty position at ASU).

My family has been especially supportive over the past two decades. My children, Chris and Carole, grew up with their Dad in school and had to give up lots of our time together so that I could study; over this time they have graduated from college and are both very interesting, independent young adults of whom I am very proud. My father-in-law for 25 years, Barrett Baldwin, was a continual source of encouragement - he never ceased to be amazed that the young Stanford psychology major he first met would some day be involved in turbulence modeling.

Most of all, I owe a great deal to my wife, Donna, for her love, humor and encouragement during the long, stressful push to complete this educational goal. She has 
selflessly given up so much time that we would like to have spent together along the ocean, and has fluffed up my sagging attitude when needed.

This has been a long 20-year journey, and indeed a rather spiritual one. Several times along the way, it would have been easy to quit. My faith in God has been a source of constant support and strength to strive onward to the finish line.

This work was performed under the auspices of the U. S. Department of Energy by the University of California, Lawrence Livermore National Laboratory under Contract No. W-7405-Eng-48. 
Dedicated to

Barrett Stone Baldwin, Jr.

(1921 - 1998) 


\section{Nomenclature}

\section{Roman Symbols}

\begin{tabular}{ll}
$f$ & Coriolis parameter \\
$g$ & gravitational constant \\
$L_{x}$ & Length of domain in x direction \\
$L_{y}$ & Length of domain in y direction \\
$L_{z}$ & Length of domain in z direction \\
$L$ & Monin-Obukhov length based on surface-layer scaling \\
$p$ & reduced dynamic pressure \\
$P$ & large-scale pressure \\
$P_{0}$ & large-scale reference pressure (100 kPa) \\
$R i_{\tau}$ & Richardson number based on friction velocity \\
$R_{f}$ & flux Richardson number \\
$R e$ & Reynolds number \\
$S_{i j}$ & resolved strain rate tensor \\
$t$ & time \\
$T$ & Temperature \\
$T_{0}$ & reference temperature (290 K for these simulations $)$ \\
$x_{i}$ & Cartesian coordinates \\
$u_{*}$ & friction velocity \\
$u_{i}$ & Cartesian velocity \\
\hline
\end{tabular}


$u$

$v$

$w$

$x_{i}$

$\mathbf{X}$

$\mathbf{y}$

$\mathbf{Z}$

$\beta$

$\delta_{i j}$

$\varepsilon_{s g s}$

$\varepsilon_{\theta} \quad$ dissipation of SGS temperature variance

$\epsilon_{i j 3} \quad$ permutation index

$\lambda_{2} \quad$ method for vortex core identification

$\Lambda \quad$ Monin-Obukhov length based on local scaling

$\nu_{T} \quad$ eddy viscosity

$\rho_{0} \quad$ reference density

$\theta \quad$ potential temperature

$\tau_{i j} \quad$ SGS stress

$\omega_{i} \quad$ vorticity component in $\mathrm{i}$ direction

$\Omega_{i j} \quad$ antisymmetric part of velocity gradient tensor 


\section{Other Symbols}

$\partial \quad$ partial derivative operator

$(-) \quad$ grid filtering operator

$(\sim) \quad$ larger grid filtering operator

Abbreviations

DNS Direct Numerical Simulation

LES Large Eddy Simulation

SGS subgrid scale 


\section{Table of Contents}

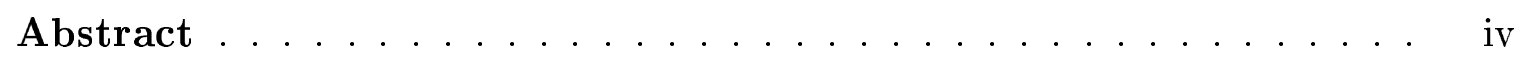

Acknowledgments ...................... vii

Nomenclature ....................

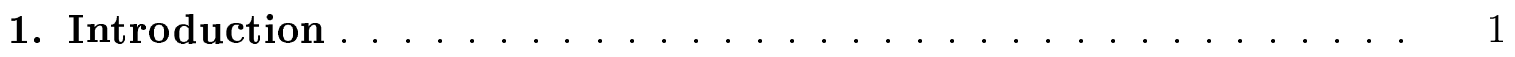

1.1. Planetary Boundary Layer Evolution . . . . . . . . . . . 3

1.2. Large-Eddy Simulation . . . . . . . . . . . . . . . 4

1.3. The Dissertation Research . . . . . . . . . . . . . . 5 5

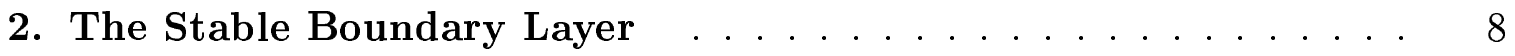

2.1. Atmospheric Observations . . . . . . . . . . . . . . 9 9

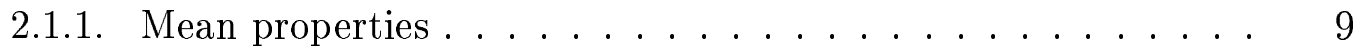

2.1.2. Turbulence properties . . . . . . . . . . . 11

2.1.3. Internal gravity waves $\ldots \ldots \ldots \ldots \ldots$

2.1.4. SBL scales and steady-state profiles . . . . . . . . . 16

2.2. Laboratory Experiments . . . . . . . . . . . . . 18

2.2.1. Organized structure in turbulent flows $\ldots \ldots \ldots \ldots$

2.2.2. Effects of stratification and shear . . . . . . . . . 21

2.2.3. Limitations of laboratory studies $\ldots \ldots \ldots \ldots$

3. Turbulence Modeling Approaches _... . . . . . . . . 56

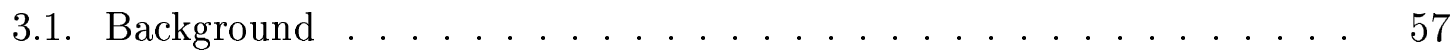

3.2. SGS Turbulence Models . . . . . . . . . . . . . . . . . 59

3.2.1. Eddy viscosity models . . . . . . . . . . . . . . 60

3.2.2. Stochastic backscatter models . . . . . . . . . . . 62

3.2.3. Scale-similaritybackscatter models . . . . . . . . . . . 63

3.2.4. Dynamic backscatter models . . . . . . . . . . . . . 64

3.2.5. Velocity Estimation Methods . . . . . . . . . . 67 


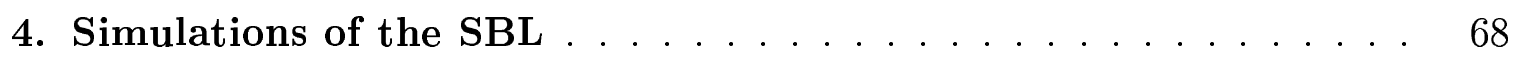

4.1. Modeling Laboratory-Scale Flows . . . . . . . . . . . . 69

4.1.1. Direct Numerical Simulation . . . . . . . . . . . . . . 69

4.1.2. Large-Eddy Simulation . . . . . . . . . . . . . 70

4.2. Modeling Atmospheric-Scale Flows . . . . . . . . . . . 71

4.2.1. Structure of the evolving SBL . . . . . . . . . . 71

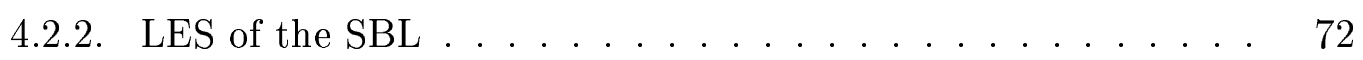

5. Numerical Formulation . . . . . . . . . . . . . . . . . . 79

5.1. Model Equations . . . . . . . . . . . . . . . . . 79

5.1.1. Governing Equations . . . . . . . . . . . . . 79

5.1.2. SGS Turbulence Model . . . . . . . . . . . . . . . . 81

5.2. The Grid and Numerical Techniques . . . . . . . . . 82

5.3. Boundary Conditions . . . . . . . . . . . . . . 84

5.3.1. Upper and Lateral Boundary Conditions . . . . . . . . . 84

5.3.2. Initial Conditions . . . . . . . . . . . . . 85

5.3.3. Lower Boundary Conditions . . . . . . . . . . . . 85

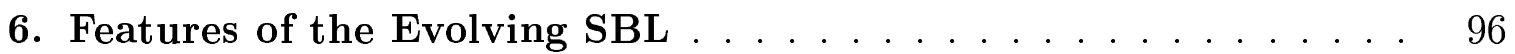

6.1. Forcing the Simulated SBL . . . . . . . . . . . . . 96

6.2. Mean Atmospheric Properties . . . . . . . . . . . . . . . . 99

6.3. Turbulence Properties . . . . . . . . . . . . . . . . 103

6.3.1. Velocity variances . . . . . . . . . . . . . . . 103

6.3.2. Temperature variances . . . . . . . . . . . . 106

6.4. An Episode of Enhanced Turbulence . . . . . . . . . . . . . 106

6.4.1. Change in Vertical SBL Structure . . . . . . . . . . 107

6.4.2. Wave-Like Undulations during the Episode . . . . . . . . 108

7. Energy Transfer in the SBL . . . . . . . . . . . . . . . 148

7.1. TKE Transfer . . . . . . . . . . . . . . . . . . . . . 148

7.2. Thermal Energy Transfer . . . . . . . . . . . . . . . . . . 149

7.3. Mixing and Stirring Efficiencies . . . . . . . . . . . 150

7.4. Waves versus Turbulence in the SBL . . . . . . . . . . 154 
7.5. Coherent Structures in the SBL . . . . . . . . . . 157

7.6. Structural Regimes of the SBL . . . . . . . . . . . 158

8. Implications for Dispersion in the SBL . . . . . . . . . . . . . 184

8.1. The Approach and LES Results Used . . . . . . . . . . . . 185

8.1.1. Source of simulated wind fields . . . . . . . . . . . 185

8.1.2. Marker particles in simulated wind fields . . . . . . . . . 186

8.2. Simulated Dispersing Plumes of Material . . . . . . . . . . . 187

8.3. Estimates of Eddy Diffusivity . . . . . . . . . . . . . 188

8.4. Discussion . . . . . . . . . . . . . . . . 192

9. Concluding Remarks . . . . . . . . . . . . . . . 209

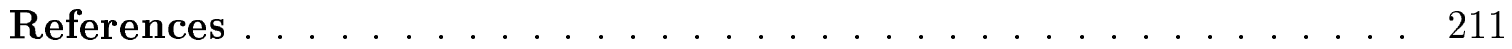




\section{List of Tables}

2.1. Summary of Length Scales for Stratified Flow . . . . . . . . 28

6.1. Summary of Simulations . . . . . . . . . . . . . . . 111

6.2. SBL Characteristics for Different Simulation Forcings . . . . . . . 112

7.1. Summary of Mixing Efficiencies for Various Mixing Mechanisms . . . 160 


\section{List of Figures}

1.1. A typical diurnal (daily) cycle of the planetary boundary layer . . . 7

2.1. Typical mean profiles of wind speed, wind direction and potential temperature for CBL and SBL . . . . . . . . . . . . . 29

2.2. Comparison of estimated SBL depth with Minnesota Experiment . . 30

2.3. Typical mean profiles of SBL at O'Neill, Nebraska, Wangara, Australia, and Haswell Colorado . . . . . . . . . . . . . . . . . . . 31

2.4. Diurnal trends observed for SBL structure . . . . . . . . . . . 32

2.5. Vertical and horizontal velocity variances observed for CBL . . . . . 33

2.6. Vertical and horizontal velocity variances observed for CBL . . . . . 33

2.7. Schematic representation of the energy spectrum of turbulence . . . . 34

2.8. Vertical profiles of dissipation rate for the SBL . . . . . . . . . 34

2.9. Measured SBL profiles from the Cabauw tower . . . . . . . . . 35

2.10. Variance spectra of vertical velocity and temperature from Cabauw tower 36

2.11. Spectra of vertical velocity and temperature from BAO tower . . . 37

2.12. Autocorrelation of vertical velocity at BAO tower . . . . . . . . 38

2.13. Temperature and vertical velocity and wave analysis from aircraft . . 38

2.14. Acoustic echo-sounder record with strong echo returns from Haswell . 39

2.15. Raw microbarograph and anemometer records from Haswell . . . . 40

2.16. Scaling regions for the SBL . . . . . . . . . . . 41

2.17. Profiles of momentum flux and temperature flux at Cabauw tower . . 42

2.18. Time series of turbulent episodes in SBL observed by Coulter . . . . 43

2.19. Profiles of average velocity variances at Cabauw tower . . . . . . . 44

2.20. Nondimensional profiles of turbulence quantities for the SBL . . . . 45

2.21. Downstream SBL evolution under three stabilities in laboratory . . . 46

2.22. Evolution map of homogeneous turbulence in stably stratified fluid . . 47

2.23. Overturning turbulent length scale vs. normalized downstream distance 48

2.24. Downstream evolution of ratio of observed to limiting overturning scales 49

2.25. Normalized vertical velocity spectra for stratified and unstratified con-

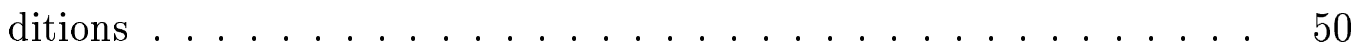


2.26. Ratio of u- and w-velocity variances vs. Richardson number Ri . . . . 51

2.27. Profiles of turbulent intensity under five stability conditions . . . . 52

2.28. Length-scale evolution map for data of Dickey and Mellor . . . . . . 53

2.29. Downstream evolution of total and turbulent vertical velocity variance 54

2.30. Normalized buoyancy flux vs. normalized downstream distance in SBL 55

2.31. Evolution of vertical KE and fluctuating PE in SBL . . . . . . . . 55

4.1. Comparison of observed SBL heights with values calculated by models 74

4.2. Evolution of friction velocity $u_{*}$ for day to night periods . . . . . . 75

4.3. Evolution of temperature and vertical temperature flux . . . . . 76

4.4. Profiles of wind speed and potential temperature at Wangara . . . . . 77

4.5. Comparison of Coleman, et al. DNS results with Nieuwstadt atmospheric SBL data . . . . . . . . . . . . . . 78

5.1. Coordinate system . . . . . . . . . . . . . . 91

5.2. Arakawa-C grid with staggered assignments for velocity . . . . . . . 91

5.3. Advection scheme of Piacsek and Williams . . . . . . . . . . . 92

5.4. Schematic illustration of aliasing effects . . . . . . . . . . . . 92

5.5. Augmentation of stress profiles using 'canopy' approach . . . . . . . 93

5.6. Comparison of stress term profiles using 'canopy' approach . . . . . . 94

5.7. Estimate of $\phi_{m}$ for NBL . . . . . . . . . . . . . 95

6.1. Simulation forcings used and maximum criteria . . . . . . . . 113

6.2. Vertical profiles of temperature and winds for SBL and CBL . . . . 114

6.3. Vertical profiles of temperature and winds for Run G7.5wt-02 . . . 115

6.4. Vertical profiles of temperature and winds for Run G5wt-02 . . . . . 115

6.5. Time history of evolving temperature structure for Run G7.5wt-02 . 116

6.6. Time history of evolving temperature structure for Run G5wt-02 . . . 116

6.7. Stability forcing index versus bulk Richardson number . . . . . . 117

6.8. Simulation forcings in terms of $B_{0}$ and $G^{2}|f| \ldots \ldots$. . . . . 118

6.9. Evaluation of Minnesota experiment SBL height algorithm . . . . . 119

6.10. Vertical profiles of velocity variances for CBL and SBL . . . . . . 120

6.11. Time history of velocity variances for Run G10.4wt-02T . . . . . . 121 
6.12. Spectra velocity variances before, during, and after SBL turbulence event in Run G10.4wt-02T . . . . . . . . . . . . . . . 122

6.13. Time history of vertically-integrated velocity variances -4 cases . . . 123

6.14. Time history of velocity variances for Run G7.5wt-02 . . . . . . . . 124

6.15. Time history of velocity variances for Run G5wt-02 . . . . . . . 125

6.16. Time history of velocity variances for Run G2.5wt-04 . . . . . . . 126

6.17. Time history of velocity variances for Run G1.5wt-02 . . . . . . . 127

6.18. spectra of $\mathrm{u}$ and $\mathrm{w}$ velocity variances for Run G1.5wt-02 at hour 7 . . 128

6.19. Time history of evolving TKE structure for Run G7.5wt-02 . . . . . . 129

6.20. Time history of evolving TKE structure for Run G5wt-02 . . . . . . . 129

6.21. Time history of evolving temperature variance for Run G7.5wt-02 . . 130

6.22. Time history of evolving temperature variance for Run G5wt-02 . . . 130

6.23. Time history of vertically-integrated velocity variances for Run $G 7.5 w t$ -

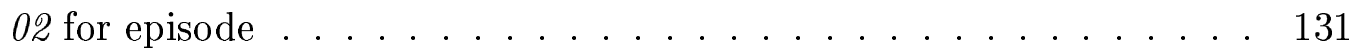

6.24. Vertical profiles of temperature and winds for Run G7.5wt-02 during

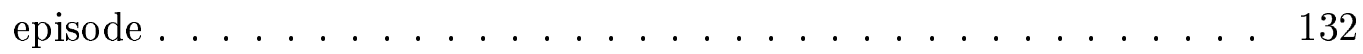

6.25. Vertical profiles of $\mathrm{u}$ and v velocity variances for Run G7.5wt-02 during episode . . . . . . . . . . . . . . . 133

6.26 . Vertical profiles of $\mathrm{w}$ velocity and potential temperature variances for Run G7.5wt-02 during episode . . . . . . . . . . . 133

6.27. Vertical profiles of stress components for Run G7.5wt-02 during episode 134

6.28. Vertical profiles of heat flux for Run G7.5wt-02 during episode . . . . 134

6.29. SGS model dynamic coefficients for Run G7.5wt-02 during episode . . 135

6.30. SGS TKE transfer for Run G7.5wt-02 during episode . . . . . . . . 136

6.31. SGS thermal energy transfer for Run G7.5wt-02 during episode . . . . 136

6.32. Temperature contours with fluctuating velocities for Run G7.5wt-02 at episode onset . . . . . . . . . . . . . . 137

6.33. Temperature contours with fluctuating velocities for Run G7.5wt-02

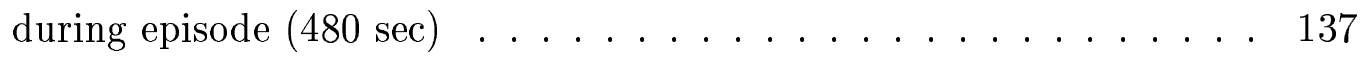

6.34. Temperature contours with fluctuating velocities for Run G7.5wt-02 during episode $(486 \mathrm{sec}) \ldots \ldots \ldots \ldots$ 
6.35. Temperature contours with fluctuating velocities for Run G7.5wt-02 during episode $(492 \mathrm{sec})$. . . . . . . . . . . . . 138

6.36. Temperature contours with fluctuating velocities for Run G7.5wt-02 during episode $(498 \mathrm{sec})$. . . . . . . . . . . . . . 139

6.37. Temperature contours with fluctuating velocities for Run G7.5wt-02 during episode $(504 \mathrm{sec})$. . . . . . . . . . . . . . 139

6.38. Temperature contours with fluctuating velocities for Run G7.5wt-02 during episode $(510 \mathrm{sec}) \ldots \ldots$. . . . . . . . . . 140

6.39. Temperature contours with fluctuating velocities for Run G7.5wt-02 during episode $(516 \mathrm{sec})$. . . . . . . . . . . . . 140

6.40. Temperature contours with fluctuating velocities for Run G7.5wt-02 during episode $(522 \mathrm{sec})$. . . . . . . . . . . . . 141

6.41. Temperature contours with fluctuating velocities for Run G7.5wt-02 near episode peak . . . . . . . . . . . . . .

6.42. Temperature isosurface showing wave-like motions for Run G7.5wt-02

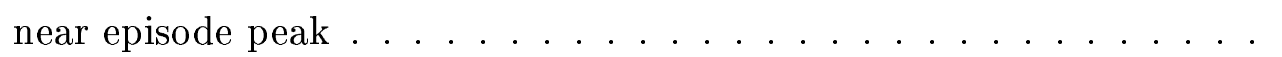

6.43. Temperature contours in $\mathrm{x}-\mathrm{z}$ plane and $\mathrm{y}-\mathrm{z}$ plane for Run G7.5wt-02 during episode $(492 \mathrm{sec})$. . . . . . . . . . . . . 143

6.44. Temperature contours with fluctuating velocities in $\mathrm{y}-\mathrm{z}$ plane for Run G7.5wt-02 near episode onset . . . . . . . . . . . 144

6.45. Temperature contours with fluctuating velocities in $\mathrm{y}-\mathrm{z}$ plane for Run

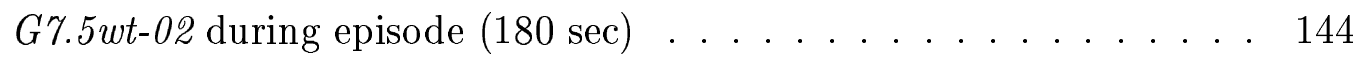

6.46. Temperature contours with fluctuating velocities in $y-z$ plane for Run

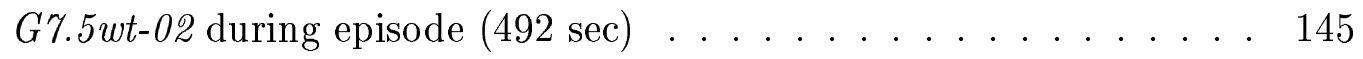

6.47. Temperature contours with fluctuating velocities in $\mathrm{y}-\mathrm{z}$ plane for Run G7.5wt-02 during episode (570 sec) . . . . . . . . . . 145

6.48. Temperature isosurface $(288 \mathrm{~K})$ showing large wave-like motions for Run G7.5wt-02 early in episode (180) sec . . . . . . . . . . . . 146

6.49. Temperature isosurface $(288 \mathrm{~K})$ showing wave-like motions for Run G7.5wt-02 early in episode (180) sec . . . . . . . . . . . . 147

7.1. Vertical profiles of SGS TKE transfer by component . . . . . . . . 160 
7.2. Vertical profiles of SGS TKE transfer by component . . . . . . . . 161

7.3. Patterns of fluctuating streamwise velocity and fluctuating temperature 161

7.4. Isosurfaces of $\theta^{\prime}=-0.45 K$ for Run $G 7.5 w t-02 \ldots \ldots . . \ldots 162$

7.5. Isosurfaces of $\theta^{\prime}=-0.5 K$ for Run G5wt-02 . . . . . . . . . 163

7.6. Vertical profile of mixing efficiency for Run G7.5wt-02 . . . . . . . 164

7.7. Vertical profile of mixing efficiency for Run G5wt-02 . . . . . . . 165

7.8. Mixing efficiency for 3 periods in Run G7.5wt-02 . . . . . . . . . 166

7.9. Brunt-Vaisala frequency during episode in Run G7.5wt-02 . . . . . 167

7.10. Normalized stirring length for episode in Run G7.5wt-02 . . . . . . 168

7.11. Stirring lengths in lower and upper $(>100 \mathrm{~m})$ SBL for episode in Run G7.5wt-02 . . . . . . . . . . . . . 169

7.12. Stirring lengths in lower and upper $(>40 \mathrm{~m})$ SBL for episode in Run

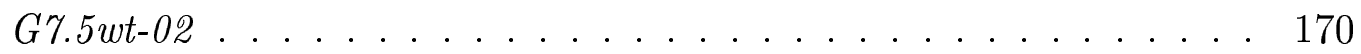

7.13. Mixing efficiency averaged over lower and upper $(>40 \mathrm{~m})$ SBL for episode in Run G7.5wt-02 . . . . . . . . . . . . . . . . 171

7.14. Phase angles at $z=45 m$ during episode in Run G7.5wt-02 . . . . . . 172

7.15. Phase angles at $z=105 m$ during episode in Run G7.5wt-02 . . . . . 173

7.16. Phase angles (degrees) at $z=135 \mathrm{~m}$ during episode in Run G7.5wt-02 174

7.17. Scorer parameter during episode in Run G7.5wt-02 . . . . . . . . 175

7.18. Isosurfaces of $\lambda_{2}=-0.001$ colored by height for Run G7.5wt-02 . . . 176

7.19. Isosurfaces of $\lambda_{2}=-0.0005$ colored by height for Run G5wt-02 . . . . 177

7.20. Four perspectives of the fluctuating velocity vectors for Run G5wt-02F 178

7.21. Fluctuating velocity vectors in x-z plane for Run G5wt-02F at hour 10179

7.22. Spectra at 3 heights for Run G5wt-02F at hour 10 . . . . . . . . 180

7.23. Cross section (x-z) of potential temperature with fluctuating velocity vectors . . . . . . . . . . . . . . . . . . 181

7.24. Total vorticity field at $\mathrm{y}=300 \mathrm{~m}$ for Run G5wt-02F . . . . . . . 182

7.25. Y-component of vorticity for Run G5wt-02F . . . . . . . . 183

8.1. Vertical profile of mean wind speed and direction used in plume study 193

8.2. Time history of vertically-integrated velocity variances . . . . . . . 194 
8.3. Vertical profiles of resolved velocity variance before and during enhanced turbulence event in SBL . . . . . . . . . . . . 195

8.4. Idealized plumes in SBL for Run G5wt-02F . . . . . . . . . . 196

8.5. Locations of marker particles for 5-minute release before SBL turbulence event for Run G10.4wt-02T . . . . . . . . . . . . 197

8.6. Locations of marker particles for 5-minute release during SBL turbulence event for Run G10.4wt-02T . . . . . . . . . . . 198

8.7. Locations of marker particles for 5-minute release into CBL for Run

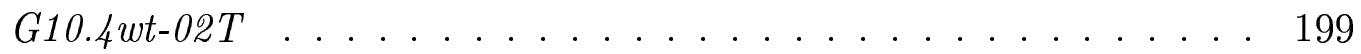

8.8. Eddy diffusivity from Equation $8.2 \ldots \ldots$. . . . . . . . . 200

8.9. Eddy diffusivity from Equation $8.3 \ldots \ldots$. . . . . . . . . 201

8.10. Eddy diffusivity from Equation 8.4 using $\Lambda$. . . . . . . . . . 202

8.11. Eddy diffusivity from Equation 8.4 using $u_{*}^{\text {local }} / N \ldots$. . . . . . . 203

8.12. Eddy diffusivity from four estimates during the episode . . . . . . . 204

8.13. Eddy diffusivity from four estimates before the episode . . . . . . 205

8.14. Eddy diffusivity from four estimates after the episode . . . . . . . 206

8.15. Estimate of $\phi_{m}$ for SBL . . . . . . . . . . . . 207

8.16. Estimate of $\phi_{m}$ for SBL using local $u_{*} \ldots \ldots \ldots$. . . . . . . 208 


\section{Chapter 1}

\section{Introduction}

The planetary boundary layer (PBL) is a complicated region to model or measure. Complications arise from diurnal variations of buoyancy effects, surface inhomogeneities, and latent heat effects, to name a few. Detailed observations of the timeand space-varying PBL structure are often quite costly to obtain and sometimes difficult to interpret. Numerical modeling allows a direct test of our theoretical understanding, since variables are under control of the researcher; this is frequently not the case for field experiments. The results of numerical modeling studies often help define field experiments, which in turn can provide the necessary data for validating numerical models. This proposal will focus on numerical modeling of selected PBL states.

Evolution of PBL structure is described by conservation equations for momentum, thermal energy, and moisture (vapor/liquid). Fluctuations of wind, temperature, and moisture occur on a wide variety of spatial and temporal scales. If a computer of arbitrarily large size were available, numerical solution of the exact governing equations would be possible (to the limit of numerical truncation errors). Currently this is possible only for a restricted class of turbulent flows at low Reynolds number, using direct or full simulation methods on high-end computers. For the PBL, with its high Reynolds number flow, solution is currently possible only for an averaged form of the governing equations that resolve the primitive variables above the averaging scale. Such averaging leads to unresolved variables, which must be evaluated in some way.

The most common approach is to close the set of equations by first-order or secondorder statistical evaluation of the Reynolds stresses, which arise from Reynoldsaveraging of the governing equations. The closure approximation must deal with all scales of turbulence which are averaged simultaneously in the Reynolds-averaged equations. Such turbulence closure is often inadequate for strongly diabatic conditions 
in the PBL.

With the availability of faster and larger computers, it is becoming more feasible to use an approach which lies between Reynolds-averaging and direct simulation. This approach is called Large-Eddy Simulation (LES). Here the governing equations are averaged in such a way that effects of large eddies are calculated directly, rather than modeled. At high Reynolds number, the turbulence may be treated as a composite of eddies having a wide range of sizes. The large eddies are viewed as containing and transporting most of the turbulent energy, while the small eddies are responsible for dissipation.

This view is consistent with observations of turbulent flows. Difficulties faced by Reynolds-stress models (i.e. anisotropy, realizability, flow-dependent effects) are associated with large-scale turbulence and are addressed explicitly in LES by the direct calculation of large eddies. The only modeling required is for the small, subgrid-scale (SGS) eddies that are easier to model (i.e. more isotropic, less flow-independent). The main advantage of the LES approach over statistical closure approaches is increased accuracy, since most of the turbulent transport is calculated explicitly, with modeling approximations at smaller scales having a less critical role. This advantage, however, is achieved at a significant increase in required computer resources over simpler models (although advanced second-order closure models also require extensive computer resources).

Because of the accuracy and detail available from LES models, results of numerical "experiments" can provide a "data base" against which to evaluate and improve simpler, more economical PBL models. These numerical experiments are less expensive than field experiments and, in many cases, provide data that are more readily interpreted in improving our theoretical understanding and modeling of the PBL. However, before such a data base can be credible, the model from which it is derived must be validated by available field and laboratory data. Another role of LES modeling is in the quantifying of uncertainty inherent in the PBL. This uncertainty arises from the stochastic nature of the PBL and is seen in the variance about estimates of mean values of $\mathrm{PBL}$ variables.

In a review of the role of LES in PBL research (Wyngaard, 1984), a pressing need was identified for extending LES to the stable boundary layer (SBL). The topic of 
the proposed research is the evolution of the SBL in response to cooling applied at the ground. The resulting stably-stratified boundary layer that develops from the ground up into the residual PBL is characterized by weak turbulence that can often be intermittent. Particular emphasis is given to the structure of turbulence during this evolution. In order to capture the stochastic (and intermittent) nature of the resulting turbulence, a large-eddy simulation (LES) approach is taken to adequately simulate the turbulence structure.

\subsection{Planetary Boundary Layer Evolution}

Typically variations in the PBL are related strongly to changes in buoyant forcing associated with changes in heating and cooling at the ground that occur through the diurnal cycle. The magnitude of the variations depends on several factors, including season of the year and cloud cover that affect the intensity of incoming solar radiation and outgoing longwave radiation, conditions at the ground that govern the response to solar heating, and the wind speed that determines the amount of mechanical mixing that reduces strong vertical temperature gradients near ground (and hence the buoyant forcing). A typical diurnal cycle of the PBL is illustrated in Figure 1.1. The heating of the ground during the day leads to convective mixing and a deep convective boundary layer (CBL). After sunset, the cooling of the ground leads to a shallow stable boundary layer (SBL) that develops from the ground up into the residual PBL structure. After sunrise, the CBL develops again, destroying the SBL. In the absence of significant heating or cooling of the ground, such as under full cloud cover, the PBL tends more toward the ideal neutral boundary layer (NBL). Strong winds will also tend to force the PBL toward the neutral state.

The evolution of the SBL on a clear night is driven by a combination of surface cooling due to longwave radiative flux divergence and a thickening of the cooled layer near the ground due to turbulent mixing. The growth rate of the SBL is related directly to the amount of shear production of turbulence, and is limited by dissipation and destruction of turbulence by buoyancy. The amount of shear-driven turbulence near the ground is parameterized by the friction velocity $\left(u_{*}\right)$. During the evolution of the SBL after sunset, the amount of shear-driven turbulence near the ground decreases 
markedly; this decrease is greater under conditions of greater surface cooling. Hence, in terms of available turbulence, the depth the SBL will increase most rapidly during the early stage of evolution; also the final depth will be less when the surface cooling is greater. The final depth of the developing SBL is related to the combined effects of surface cooling and shear-driven turbulence.

Because the CBL is formed by strong vertical mixing due to the convectivelydriven thermals, the vertical gradients of temperature, moisture, and winds are relatively small. In contrast, the weak turbulent mixing in the SBL leads to large vertical gradients. With the largest gradients near the ground, the turbulence is greatest there as well. In order to simulate the turbulence in the SBL properly, adequate consideration must be given to the region near the ground.

Certain characteristics of the SBL complicate the modeling of its structure and evolution, especially compared with the CBL. The SBL is generally non-steady throughout the night, although it undergoes rapid development after sunset, followed by more gradual development. The diffusion time scale for the SBL is about 30 hours, compared to about 10 minutes for the CBL (Brost and Wyngaard, 1978). Thus it takes a long time for the mean fields to respond to changes within the domain. In strongly stable conditions, turbulence may become intermittent or episodic. The large scale, low frequency variations are unique and do not repeat in any consistent fashion (Hunt, 1985). Turbulent mixing, especially in the vertical, can be reduced by stable stratification to the point where vertically adjacent horizontal layers become decoupled. The lack of strong turbulence provides the SBL with a long "memory", so that turbulence can exist at locations far removed from the generation source; thus local conditions may not be adequate for parameterizing turbulence.

\subsection{Large-Eddy Simulation}

The modeling difficulties associated with the SBL generally preclude the use of simple approaches. For example, intermittency of turbulence in strongly stable conditions confounds traditional modeling approaches, such as Reynolds-averaged models. Large-eddy simulation (LES) provides a suitable alternative, if the intermittent features of the turbulence can be resolved by the explicitly calculated scales of motion. 
A requirement of LES is that the numerical grid must be fine enough to capture the large eddies. In the case of the CBL, this is not a problem since the convectivelydriven eddies are quite large, and most of the turbulent transport is resolved by modest grids. In the case of the SBL, the grid must be quite fine, especially near the ground; at the same time, the modeling domain must be sufficiently large to capture the large-scale features that impact the behavior of the SBL. For LES to be successful in the SBL case, energy-containing eddies must be resolved. Otherwise the SGS eddy model is being tasked to model too much of the turbulent motion, and the advantage of LES is lost.

\subsection{The Dissertation Research}

LES studies of the SBL are a relatively new research effort, when compared with the numerous LES studies of the CBL. There are several dissertation research topics available in this area. A hurdle that may have deterred research activity in this area is that LES of the SBL puts relatively high demands on computational resources due to: (1) the need to resolve energy-containing eddies which occur at smaller and smaller scales as stable stratification increases, while maintaining an adequate simulation domain, which leads to a larger number of grid cells, (2) the associated decrease in size of integration time step with smaller grid cells, and (3) the non-steady character of the SBL, necessitating long simulation periods for stationary statistics. Since computer resources are somewhat limited, the SGS turbulence model must have some ability to treat eddies of importance to the flow. Hence the choice of SGS turbulence model is a critical aspect of the research. In addition to the higher computational burden for LES of the SBL compared with that of the CBL, our understanding of the SBL has been limited by a relative scarcity of turbulence observations in the SBL. Thus validation of LES results for the SBL is limited. Nonetheless, if successful, LES of the SBL can serve as a "numerical laboratory", providing "data" where observations are lacking.

The goal of this dissertation research is more realistic simulation of the SBL which requires use of a SGS turbulence model that adequately represents the generation, transfer, and dissipation in stably-stratified, high Reynolds-number flows. Previous 
research, to be described in subsequent chapters, has shown that turbulent energy flows not only from larger to smaller scales, but also from smaller to larger scales (energy backscatter). The SGS model used here has an energy backscatter capability that is responsive to the effects of thermal stratification. The SGS model is incorporated in an established LES model methodology.

Simulations cover the evolution of the SBL in response to a range of forcing conditions specified in terms of the geostrophic wind and the surface cooling. The environment is simplified by being cloud-free and over flat terrain. Analyses presented here seek to address the following questions:

1. How is the turbulence modified in the presence of increasing stable stratification as the SBL develops during the night?

2. What are the different structural characteristics of the SBLs that develop in response to different meteorological conditions (geostrophic wind and surface cooling)?

3. Can episodic turbulence events that occur in the SBL be simulated using LES and, if so, what impacts do such events have on dispersion of airborne material? 


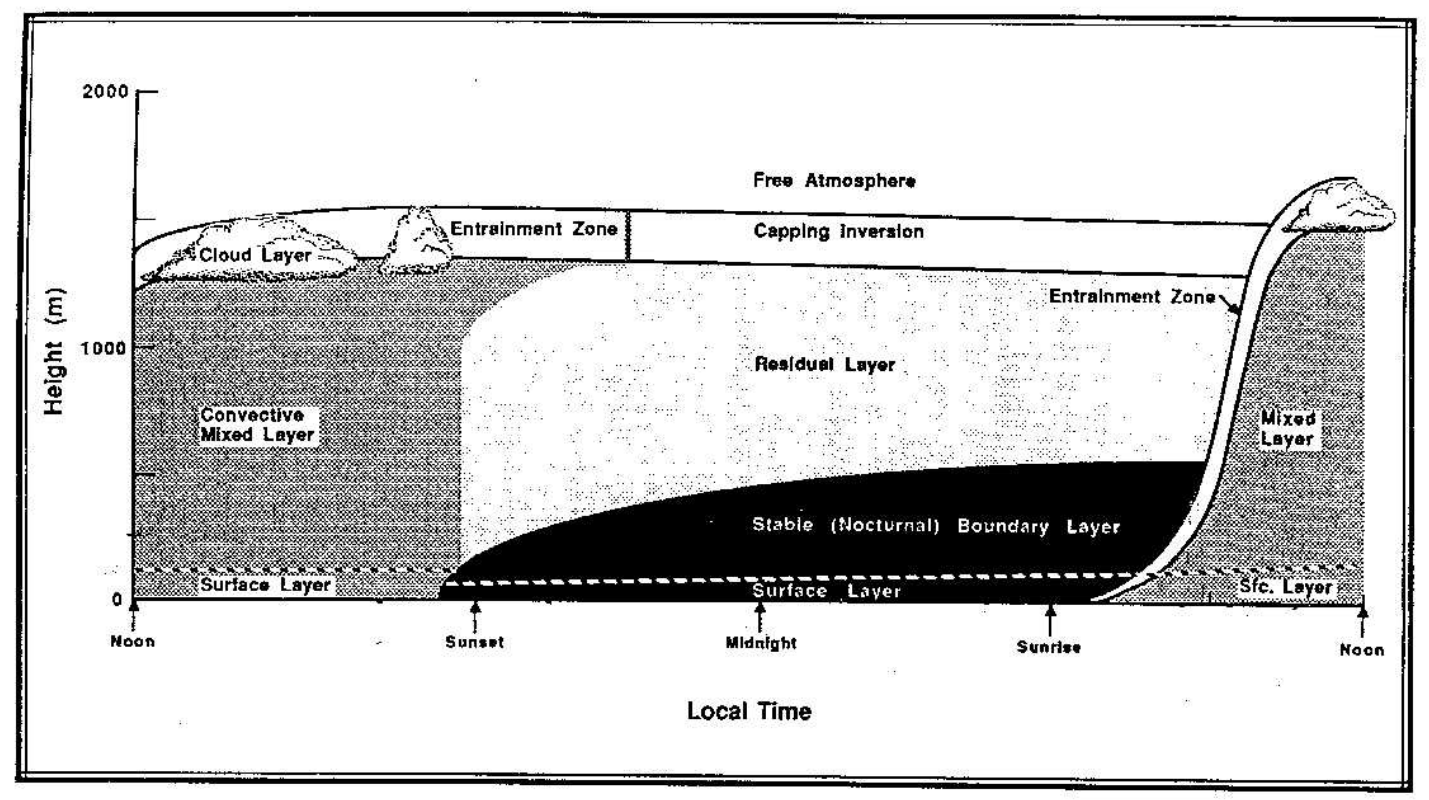

Figure 1.1: A typical diurnal (daily) cycle of the planetary boundary layer in high pressure regions over land (after Stull, 1988). 


\section{Chapter 2}

\section{The Stable Boundary Layer}

The planetary boundary layer $(\mathrm{PBL})$ is that portion of the atmosphere nearest the earth's surface and which, consequently, is modified by surface features such as roughness, terrain, moisture, and temperature. The large-scale flow, usually characterized by the geostrophic wind, is retarded by frictional effects of the surface. The resulting velocity profile, in the absence of buoyancy effects, is the classical Ekman spiral. Although the atmosphere is rarely without some level of buoyancy effects, it is useful to consider the neutrally buoyant case because there is a wealth of theoretical modeling and experimental work on neutral boundary layers.

Before going on, some discussion of atmospheric stability is in order. In the laboratory, a common measure of the stability is static stability: $-\partial \rho / \partial z$ in an incompressible fluid. In the atmosphere, the vertical temperature gradient is a more common measure, since there is most often some amount of temperature stratification. To account for adiabatic heating and cooling due to pressure changes, potential temperature $(\theta)$ is used (see Equation 5.1). If $\theta$ is constant with height, air displaced vertically will develop no buoyancy; hence the stability is "neutral". If $\theta$ decreases with height, air displaced upward (downward) will be warmer (cooler) than the surrounding air and will tend to continue to rise (fall) due to its positive buoyancy; hence the air layer is "unstable". If, on the other hand, $\theta$ increases with height, vertically displaced air will develop negative buoyancy and will tend to return to its original height; the layer is "stable" or stably-stratified.

The stably-stratified PBL occurs most frequently at night. It is helpful to consider the SBL in the context of the wide range of atmospheric boundary layer states that occur during the diurnal (daily) cycle. Throughout the cycle, there are varying degrees of departure from the ideal neutral state due to buoyancy, associated usually with surface heating or cooling. During the day, the earth's surface is heated by the sun and typically becomes warmer than the overlying air. In the resulting unstable layer of 
air, turbulence develops spontaneously and is called "convective"; hence, the typical daytime boundary layer is referred to as the convective boundary layer (CBL). At night, radiative cooling at the earth's surface leads to an overlying layer of stable air that constitutes the SBL. For short periods of time during the evening or early morning, the PBL is in transition and may contain multiple layers with differing stability properties, i.e. a shallow unstable layer under a stable layer as the sun begins to heat the earth's surface in the morning. If, during the day or night, wind speeds are high, the resulting mechanical turbulence due to wind shear will tend to reduce the magnitude of the temperature stratification and the boundary layer will tend towards neutral. In conditions where the surface is neither warming nor cooling (i.e. cloudy skies), a neutral boundary layer (NBL) will result.

In the subsections that follow, the SBL will be described in more detail. The discussion will draw upon atmospheric and laboratory observations, as well as theoretical and modeling studies, and will attempt to summarize to some degree our current understanding of the SBL. The focus will be on the cloudless PBL above flat terrain. This situation is best understood, as the presence of clouds and topography can greatly complicate PBL structure and behavior.

\subsection{Atmospheric Observations}

\subsubsection{Mean properties}

Many of the observational studies of the PBL have been motivated by a need to understand the transport and diffusion of hazardous materials released to the atmosphere from near the surface. A wealth of data has been collected near the surface, usually from towers. Tethered balloons and, more recently, remote sensing have provided more detailed measurements of the upper portion of the PBL, previously measured only by aircraft. The daytime CBL has been measured the most, and is discussed here for purposes of comparison with the SBL. Measurement of the SBL has been complicated by the low levels and intermittency of turbulence, and the presence of both turbulence and gravity waves in the mean flows.

The mean profiles of wind and temperature for the CBL are shown in Figure 2.1a. 
The vertical gradients are small, except near the surface, indicating the well-mixed nature of this PBL state. The height of the CBL is often well defined by a capping temperature inversion. Above there is an entrainment zone where a transition occurs in wind and temperature structure to the large-scale flow aloft. The CBL height is typically 1000-2000 $\mathrm{m}$ and generally increases with increased surface heating and the subsequent convective vertical motion; however, the CBL height tends to decrease with increased wind speed and the subsequent turbulent mixing which damps the convective activity.

In contrast, the mean profiles of the SBL (shown in Figure 2.1b) have larger vertical gradients. Of particular note is the local maximum (jet) in wind speed and strong directional shear near the top of the SBL; these have important implications for the behavior of gravity waves. The height of the SBL is difficult to characterize; typically it can be an order of magnitude smaller than the CBL height. The stable stratification that results from surface cooling tends to restrict eddy size and vertical turbulent transfer, leading to decoupled layers (Nieuwstadt, 1984a); in the SBL the direct effect of surface cooling on upper layers becomes negligible. Increased wind speed can again couple layers due to turbulent mixing, and increase SBL height. This relation of wind speed and surface cooling to SBL height is shown in Figure 2.2. Increased mechanical mixing (represented by the friction velocity) due to increased wind speed tends to increase SBL height; this increase is countered, to a lesser degree, as the surface cooling becomes larger. Rarely is there a strong demarcation to mark the top of the SBL; a common choice is where the local heat flux drops to $5 \%$ of its surface value (Caughey, et al, 1979). Mean profiles from other field studies are shown in Figure 2.3. Even though the SBL heights are quite different, the profiles have similar features. All are characterized by a low level jet near the top of the SBL which is most pronounced for the SBL with the strongest temperature gradient.

The time behavior of the CBL height is understood better than that for the SBL. Figure 2.4a shows the typical rise of the CBL height through afternoon to an asymptotic level. The behavior of the SBL for a specific case study is shown in Figure 2.4b. Unlike the CBL which develops steadily through the day to an asymptotic level, the SBL height achieved shortly after sunset is close to its equilibrium value; however, the structure within the SBL takes a long time to reach equilibrium (Holtslag and 
Nieuwstadt, 1986). Combining the time behavior of the CBL and SBL heights gives an approximation of the diurnal cycle for the PBL, as shown in Figure 2.4c. The deep CBL that develops during the day is undercut by the shallow SBL that develops after sunset. The CBL and SBL are discontinuous, with remnants of the CBL remaining aloft above the SBL. After sunrise, the developing CBL erodes the SBL and quickly rises above the SBL height.

\subsubsection{Turbulence properties}

The vertical profiles of turbulence quantities also have characteristic shapes for the CBL and SBL. Profiles of normalized velocity variances for the CBL are shown in Figure 2.5( $\mathrm{a}$ and $\mathrm{b}$ ). The variance of vertical velocity (Figure 2.5a) increases with height, reflecting the increase in size of convective eddies as one moves away from the ground up to about half the depth of the CBL. The variances of horizontal velocity components (Figure 2.5b) are relatively constant with height, since the ground does not constrain horizontal eddy motion as it does the vertical eddy motion. The thermally dominated turbulence remains strong throughout the CBL and supports the high level of mixing that has led to the CBL often being called the "mixed" layer.

In contrast, the SBL is dominated by mechanical turbulence that is strongest near the ground. Profiles of normalized velocity variances for the SBL, given in Figure 2.6, show a distinct decrease with height. This contrast is especially notable for the vertical velocity, compared with an increase with height in the lower half of the CBL; effects of stratification are seen most clearly in the vertical velocity fluctuations. It should be noted that profiles in both Figures 2.5 and 2.6 are normalized by a constant surface parameter. Although the parameter is different for the CBL and SBL, the profile shapes may be compared. The profiles in Figure 2.6 represent turbulence only. In the cases where gravity waves coexist with turbulence in the SBL, there are elevated local maxima of variances, especially for the vertical velocity; this issue will be discussed in the next subsection.

Spectra of the velocity components give much valuable information. First, they show how the turbulent energy is distributed across different ranges of frequencies as represented schematically in Figure 2.7. Turbulent energy extracted from the mean 
flow appears at the low frequency end of the spectrum. The energy then "cascades" to higher frequencies as turbulent mixing distributes energy to smaller and smaller scales of motion. Finally, at the high frequency end of the spectrum, energy is dissipated into heat by viscous action at scales approaching the molecular level. The typical spectra for daytime convective conditions has a spectral shape in the cascade region (inertial subrange) of $-5 / 3$, i.e. $E(k) \sim k^{-5 / 3}$.

Secondly, the peak in the spectrum provides the spectral length scale $\lambda_{m}$, for the particular velocity component, giving a measure of the size (in the direction of the velocity component, of the most energetic eddies. Measured vertical profiles of $\lambda_{m}$ from velocity spectra in the CBL show an increase with height of $\lambda_{m}$ for $\sigma_{w}^{2}$ in the lower half of the CBL consistent with Figure 2.5a. Profiles of $\lambda_{m}$ for $\sigma_{u}^{2}$ and $\sigma_{v}^{2}$ are nearly constant with height in the CBL consistent with Figure 2.5b. For the SBL, the profiles of $\lambda_{m}$ are not consistent with the profiles of velocity variance (in Figure 2.6). Instead, all the SBL spectral length scales increase with height, similar to the $\lambda_{m}$ profile for $\sigma_{w}^{2}$ in the CBL, but not for $\sigma_{u}^{2}$ and $\sigma_{v}^{2}$; this suggests that near the ground both the horizontal and vertical scales of energy-containing eddies tend to be reduced in the SBL.

Thirdly, spectra give an estimate of the dissipation rate, $\varepsilon$. Vertical profiles of $\varepsilon$ for strongly convective conditions have very little decrease with height when compared with profiles for weakly convective (near-neutral) and stable conditions (as shown in Figure 2.8 for the SBL).

\subsubsection{Internal gravity waves}

A complicating feature of the SBL is the occasional presence of internal gravity waves (IGW). These waves mingle with the turbulence and can be quite difficult to differentiate. What follows are results from field experiments in the SBL that illustrate various characteristics of IGW.

A 200-m tower in rather ideal surroundings (flat, homogeneous) near Cabanw in the Netherlands has been instrumented at 6 levels. Wind and temperature profiles for the study period are shown in Figure 2.9a (from DeBass and Driedonks, 1985); they illustrate both the wind shear and stable stratification requisite for IGW to form. The 
profile of vertical velocity variance, Figure $2.9 \mathrm{~b}$, has a primary elevated maximum well up into the SBL and a secondary maximum near the ground. The primary maximum aloft is in distinct contrast to the typical profile shown in Figure 2.6. The profile in Figure $2.9 \mathrm{~b}$ appears to come from a combination of processes: turbulence producing a maximum variance near the ground and a second process aloft producing even greater fluctuations in the vertical velocity; this second process is thought to be IGW.

Inspection of the time history of temperature at the 6 levels, Figure 2.9c, supports the proposed presence of IGW. In the SBL with a strong vertical temperature gradient, large fluctuations in temperature are associated with large amplitude wave-like motions. This is especially evident at the 120-m and 160-m levels, and corresponds with the large increase in vertical velocity variance in Figure $2.9 \mathrm{~b}$. The character of the large temperature fluctuations is not steady in time, having a period of especially large amplitude fluctuations from 0120 to 0130 . The temperature traces at $20 \mathrm{~m}$ and $200 \mathrm{~m}$ have the lowest amplitude fluctuations, but the frequencies are quite different from one another. At $20 \mathrm{~m}$, the fluctuations are mainly high frequency (turbulence) superimposed on mid- range frequencies; however, at $200 \mathrm{~m}$, the turbulence component is absent, leaving only the wave-like motion. This is corroborated by the vertical velocity spectra for the 6 levels shown in Figure 2.10. At $20 \mathrm{~m}$, most of the energy is at the high frequency end, while at $200 \mathrm{~m}$, the energy is concentrated at the middle range of frequencies. The greatest amount of energy is at $120 \mathrm{~m}$ and $160 \mathrm{~m}$ levels, with the peak at the frequency of the probable IGW. The dominant period of fluctuations in Figure 2.9c is about $100 \mathrm{~s}$, corresponding to the frequency of peak energy of $0.01 \mathrm{~s}^{-1}$.

A second experiment was performed at the 300-m BAO tower, located $30 \mathrm{~km}$ east of the Rocky Mountain foothills in rolling terrain; the tower is instrumented at 8 levels. Three selected periods contain IGW characterized as (a) weak, (b) moderate, and (c) strong. In general, the wind speeds are higher than in the Cabauw 30 May experiment, with stability decreasing with height within the first $100 \mathrm{~m}$. The spectra of vertical velocity, Figure 2.11a-c, show that the IGW are once again at higher levels in the SBL. Spectra at $10 \mathrm{~m}$ have their peak at high frequency (turbulence), while those at $150 \mathrm{~m}$ are at lower frequencies. As the IGW become stronger, the energy increases and the peak shifts to lower frequencies. Although time traces were 
not available, the temperature spectra for $150 \mathrm{~m}$ (also shown in Figure 2.11a-c) indicate increased fluctuations, especially at lower frequencies, as IGW become stronger. Autocorrelation functions for vertical velocity in the strong IGW case at 3 levels is shown in Figure 2.12. Near the ground, the autocorrelation function falls off most rapidly. At higher levels, the drop off is less rapid and a secondary maximum occurs at a period of about $100 \mathrm{~s}$; this would appear to be the dominant period of the IGW and corresponds to the period of IGW observed at the Cabauw tower, discussed previously.

Considered next are data measured in the elevated marine temperature inversion that exists along the California coast in summer. Although this inversion is not an example of the SBL starting at the ground, it does illustrate the overturning in thin layers associated with IGW similar to those observed in the upper portions of the SBL. It should be noted that the California coastal waters associated with this elevated inversion are quite cool and thus the inversion lies above a neutral to slightly stable boundary layer (Metcalf, 1975). The inversion is a transition zone between warm, dry air aloft associated with subsidence around the Pacific High, and the cool, moist marine air near the surface. In the experiment of interest (Metcalf and Atlas, 1973), aircraft flights were made through the inversion layer. Wind and temperature data along the flight path are shown in the lower portion of Figure 2.13. In the upper portion, the addition of supporting data allows a sketch of the wave structure in the inversion. The overturning of the IGW creates patches of local thermal instability, indicated by shading. The mean wind speed was about $2-3 \mathrm{~m} / \mathrm{s}$, giving a wave period of 30-50 s for the observed lengths of about $100 \mathrm{~m}$. This is about half the period of IGW discussed previously.

The final experiment discussed here was performed on a 150-m tower at Haswell in the plains of southeastern Colorado; the site has a horizontally homogeneous fetch of the sparse buffalo grass. Wind and temperature measurements were made at the tower top and bottom and on a vertically moving carriage, and an acoustic sounder was located nearby. This study shows clearly the generation and apparent breaking of IGW (Hooke, et al, 1973). The acoustic sounder record in Figure 2.14 shows distinct wave motion beginning after 1705 LT and breaking around 1715 LT in the layer between about 100 and $150 \mathrm{~m}$ height. The wind profile measured during the 
instrument carriage ascent (ending at 171S LT) has a great deal of variation in the 100-150 m layer that is missing (or smoothed out) by the time the carriage descends (after 1721 LT). The smoother profile may well be due to turbulent mixing from IGW. Traces of pressure, wind speed, and anemometer elevation angle at the tower top (Figure 2.15) display the characteristic wave-like fluctuations around 1710 LT. These fluctuations suddenly increase in frequency around 1715 LT in an apparent shift from waves to turbulence, i.e. breaking waves. Also included in Figure 2.15 is the trace of elevation angle near the surface. During the IGW episode, there is an increase in turbulence intensity near the ground. The wave period deduced from the acoustic echo-louder record and tower data was about 100 seconds, consistent with the observations on the BAO and Cabauw towers.

In summary, atmospheric observations show that the turbulence and depth of the SBL are much less than those for the CBL. While the CBL is constantly dominated by large convective eddies that may be treated stochastically, the SBL is complicated by the intermittent presence of gravity waves, especially in the upper portions of the SBL. These waves mingle with the turbulence and make analytic treatment of velocity and scalar fluctuations more difficult. IGW can create significant fluctuations at midrange frequencies $(\sim 0.01 \mathrm{~Hz})$ and occasionally shift dramatically to higher frequency (turbulence) as they break. IGW are usually found in thin layers (20-50 m thick) and rarely affect the entire SBL as convective eddies do in the CBL.

Coulter (1990) documented a noctural period (2 Oct 1985) where several turbulence episodes occurred. He used a minisodar, and observed Kelvin-Helmholtz waves and instabilities having 2-minute periods that developed and penetrated the top of the SBL in approximately 1 hour cycles, as shown in Figure 2.18a. He analyzed turbulence properties in terms of vertical velocity RMS, and TKE and temperature variance dissipation rates, and found that these quantities increased together during the turbulence episodes (see Figure 2.18b). The night before did not have such turbulence episodes, even though the temperature gradient was similar to October 2. 


\subsubsection{SBL scales and steady-state profiles}

The scales suitable for the SBL vary with the degree of stratification. The continuum of SBL states as characterized by the Monin-Obukhov (M-O) length (L) can be broken down into height-dependent regions that have their own unique scales, as shown in Figure 2.16. Surface layer similarity scaling is applicable to the lowest $10 \%$ of the SBL throughout a wide range of stratification; under very stable conditions, the turbulence becomes intermittent. Above the surface layer, local scaling applies in less stable regions, while "z-less" scaling applies to the more stable conditions. In local scaling, local values of heat and momentum flux are used to derive a local MO length $(\Lambda)$ to be used in place of $\mathrm{L}$ in surface layer similarity expressions. For $z / \Lambda>1$, vertical motions are sufficiently inhibited that the influence of the ground is negligible; at this point, dimensionless variables become constant with height (i.e., $\mathrm{z}$ is no longer a relevant length scale). No suitable scales are available for the region of intermittent turbulence.

Local scaling allows extension of well-known similarity formulations from the surface layer up into the rest of the SBL. Z-less scaling is essentially local scaling with only one relevant length scale, $\Lambda$, derived from local flux values. In both scalings, the unavailability of values of local fluxes above the surface layer can present a difficulty. To overcome this, analytical profiles of heat and momentum flux can be developed based on observations. Nieuwstadt (1985) proposed flux profiles of $\overline{w \theta} / \overline{w \theta_{0}}=(1-z / h)$ (see Figure 2.17a) and $\tau / u_{*}^{2}=(1-z / h)^{3 / 2}$ (see Figure 2.17b) that were consistent with the Cabauw data. The resulting velocity variances had a $(1-z / h)^{3 / 2}$ dependence. These profiles, shown in Figures 2.19a-c, are in reasonable agreement with the data; however, the variability about the average values, indicated by the horizontal error bars, makes the agreement less conclusive. The corresponding profile for $\overline{v^{\prime 2}}$ by Wyngaard (1975), as shown in Figure 2.20, matches the shape of the average Cabauw data better; the $\overline{u^{2}}$ profile, however, does not match as well. The resulting TKE production and dissipation profiles of Nieuwstadt (1985) had a $(1-z / h)$ dependence.

The time scales of the SBL are quite different from those for the CBL. The diffusion time scale for the SBL is about 30 hours, compared with about 10 minutes for the CBL (Brost and Wyngaard, 1978). Thus it takes a long time for the mean fields to respond to changes within the domain. If boundary conditions are held constant, a steady 
state will eventually be reached; however, such constant conditions are not realistic for the nocturnal environment. Although the mean and turbulent SBL structure may be non-steady, the turbulence structure evolves in a self-similar manner that depends on local, characteristic parameters and has a response time that is much smaller than that for the mean fields (Nieuwstadt, 1981). The time history of the flow has a direct impact on the mean flow structure, but only an indirect impact on turbulence structure through the relation of local, characteristic parameters to mean flow structure.

A primary scale for SBL structure is the height $h$. Because the SBL evolves throughout the night, the height is observed to increase rapidly during the first few hours after sunset and then increase more slowly (see Figure $2.4 \mathrm{~b}$ ); the growth profile suggests that $h=f\left(t^{1 / 2}\right)$, where $\mathrm{t}$ is the time since the surface began cooling. In spite of the obvious time-variation of $h$, numerous studies have addressed the steady-state value of $h$, using diagnostic models. From the classical Ekman layer with constant eddy diffusivity $\mathrm{K}, h=c(K / f)^{1 / 2}$. K may be calculated as the product of a velocity scale $q$ and a length scale $l$. Estimating $q \sim u_{*}, l \sim h$ (neutral), and $l \sim L$ (very stable), the neutral height $h=c u_{*} / f$ and the stable height $h=c\left(u_{*} L / f\right)^{1} / 2$ (first derived by Zilitinkevich, 1972). Nieuwstadt (1981) proposed a diagnostic model that sought to match both the neutral $h=0.3 u_{*} / f$ and stable (Zilitinkevich) $h=0.4\left(u_{*} L / f\right)^{1 / 2}$. His model, $h=\left(0.3 u_{*} / f\right) /(1+1.9 h / L)$, matches the Cabauw data for $u_{*} / f L<40$, but underpredicts the observed $h$ for more stable conditions $\left(u_{*} / f L>40\right)$. Arya (1981) fit the stable and neutral expressions for $h$ to the Cabauw data, with the resulting $h=0.74\left(u_{*} L / f\right) \sim Z$ and $h=15 u_{*} / f$. Unfortunately the scatter was too large for there to be much confidence in the parameters determined from the fit. Venkatram (1980) proposed a simplification of the Zilitinkevich model by expressing L in terms of $u_{*}^{2}$ that led to $h=c u *^{3 / 2} / f$, where $\mathrm{c}$ is about 20 based on the Minnesota data.

Steady-state results of prognostic SBL models have been used to evaluate diagnostic expressions for h. For barotropic conditions, Brost and Wyngaard (1978) found that $h=f\left(u_{*}^{2}\right)$ worked well, but not for baroclinic conditions. For the Zilitinkevich expression, their results gave a value of 0.4 for the constant; Wyngaard (1975) had previously found a value of 0.22 for that constant. In both models, constant cooling was used to achieve steady-state results. 
Prognostic models will be discussed in Chapter 4. Such models are more applicable to the non-steady SBL than diagnostic models (Deardorff, 1972b), but unfortunately they are much less practical. A point should be made here about model-data comparisons for SBL heights. Diagnostic models are highly correlated through $u_{*}$ and L and have been found to explain only a part (25\%) of the total variance of observed SBL heights (Mahrt and Heald, 1979). Prognostic models that are initialized with observations have a built-in explanation of observed variance; Andre (1983) found that most of the variance $(84 \%)$ comes from night-to-night variation in observed $\mathrm{h}$ rather than from the within-night variation that the prognostic models seek to explain.

\section{$2.2 \quad$ Laboratory Experiments}

The previous subsection summarized observations of the PBL. Of course, all the relevant processes are at work in field studies such as these. However, because complete and representative measurement of all processes is not generally possible, one is left with the difficulty of interpreting such measurements. In contrast, the laboratory provides a controlled environment for studying processes of interest at a level of detail that is often much more costly to do in the actual environment. For those processes that are included in the "idealized" simulation done in the laboratory setting, one can specify values associated with certain processes while measuring the response of another process. In this way, the interaction of a selected set of processes can be quantified; a rather complex example is the laboratory study of wave-turbulence interaction at various levels of shear and stratification (Weinstock, 1984). The disadvantage of the controlled, laboratory environment is that it may not be possible to include all processes relevant to a particular environment of interest. For example, the laboratory is limited in the range of scales that may be simulated, and hence the results can be difficult to apply to the complex conditions of the PBL (Pao, 1973).

\subsubsection{Organized structure in turbulent flows}

Measurements made in mixing layers and boundary layers with rather simple geometries strongly suggest that turbulent flow can be decomposed into "quasi-deterministic", 
unsteady large-scale motion and small-scale random fluctuations (Knight and Murray, 1980). The large-scale structure appears to interact with the mean flow, having a controlling influence on mean flow properties through entrainment by large eddies; small eddies do not appear to affect the the large-scale structure, even at high Reynolds number (Roshko, 1976). In a developing mixing layer, the geometry and distribution of large-scale features has been found by Roshko to vary linearly with layer thickness, and thus with distance downstream of the splitter plate that generates the layer. In more complex mixing situations, large-scale structure becomes less deterministic as measurements at a given point tend to reflect several different structures at various stages of development; in boundary layers, this structure is harder to find in the presence of three-dimensional, multiple-scale eddy motion and where effects of Reynolds number are more pronounced (Laufer, 1975).

Methods used to study organized structure have been reviewed by Cantwell (1981). The approaches fall generally into two categories: statistical correlation methods and flow visualization methods. Statistical correlations give very quantitative information about the flow, but often miss important details and can be quite unphysical. Favre space-time correlations are an improvement, in that they add phase information. A more physical description is obtained through flow visualization. This method is especially useful in providing insight into flow behavior. This information is only semiquantitative and can be ambiguous (and even misleading when naively interpreted). In the end, both types of methods are needed and compliment each other.

Four main constituents of organized structure observed in turbulent boundary layers have been summarized by Cantwell (1981). Near the wall, there is a thin layer of streamwise vortices. Outside this layer is another in which intense small-scale motions or "bursts" occur. In the outer layer, there are both intense small-scale motion and large-scale structures or "bulges". This is a rather simplistic picture of turbulent flow, but it identifies dominant structures within the boundary layer relative to location from the wall.

Much experimental work has been done to investigate the bursting process that occurs between the wall layer and the outer layer. The mechanism for initiating bursts seems to be related to intense shear and local instability, created when large eddies from the outer layer impinge on the wall layer. The rate and intensity of bursting 
increases with increased Reynolds number, and with adverse pressure gradients; all the net turbulent energy near the wall is observed to occur during bursts (Cantwell, 1981). These bulges are conjectured to become rather passive, so that only new bursts traveling outward from the wall maintain Reynolds stress and growth of the outer layer (Kovasznay et al., 1970). The triggering of these bursts by the "scouring" action of large eddies may be the link between quasi-deterministic, large-scale structure and the production and maintenance of turbulence (Cantwell, 1981).

Consensus is being reached on some aspects of ordered large-scale structure (and even small-scale structure) in turbulent flow for simple mixing layers and boundary layers. Most of the turbulent energy near the wall occurs in bursts; the mean period between bursts scales with the outer flow and reflects the burst production rate, propagation, and decay (Cantwell, 1981). Although streamwise structure becomes less deterministic with time, spanwise structure persists after the mixing layer reaches an asymptotic state; this suggests that, at equilibrium, the flow contains large-scale spanwise structure with small-scale turbulence superimposed (Bernal et al., 1980). It is probable that large-scale structure in mixing layers is not the same as that in boundary layers (Bradshaw, 1980). Nevertheless, the same theoretical and analytical constructs may well apply to both.

Several aspects of organized structure are still controversial. The details of interaction between outer and inner layers in boundary layers are difficult to assess because of the elliptic nature of the fluid motion. There is increasing evidence that the energy "cascade" occurs in both directions between large and small scale. A strong energy transfer has been observed from the mean flow to large eddies during vortex pairing; this is followed by a reverse transfer of energy during the subsequent relaxation period (Knight and Murray, 1980). Buoyancy is conjectured to affect the bursting process, but details of an interaction mechanism between buoyancy and bursting have not been established (Fukui, et al., 1983). In evaluating buoyancy effects, distance from the wall is a critical parameter (Arya and Plate, 1969). 


\subsubsection{Effects of stratification and shear}

The primary feature of the SBL is temperature stratification leading to positive static stability. The laboratory offers an excellent opportunity to quantify the effects of stratification on turbulence in the flow; use of the word stratification hereafter refers to stable stratification. Parameters to be discussed that reflect buoyancy effects include turbulence length scales, spectral features, amount of anisotropy, and the fraction of total kinetic energy partitioned into potential energy (waves).

The evolution (in time and space) of selected length scales, characterizing stablystratified turbulent flows, provides a conceptual framework for quantifying the effects of buoyancy. Work done in the ten-layer, salt-stratified, closed-loop water channel at UCSD by Van Atta and colleagues has provided quantification of length-scale evolution under varying degrees of stratification. The length scales that best describe the effects of buoyancy are given in Table 2.1, where $\varepsilon$ is the dissipation rate, $N$ is the Brunt-Vaisala frequency, (defined by $\left.N^{2}=-(g / \bar{\rho}) \partial \rho / \partial z\right), \bar{\rho}$ is horizontallyaveraged density, $\rho^{\prime}$ is the root-mean-square (rms) density fluctuation, and $w^{\prime}$ is the rms vertical velocity fluctuation. The Ozmidov scale $L_{R}$ is used to represent the upper bound of eddy size in a stably-stratified flow, where buoyancy limits the growth of eddies. At the other end of the spectrum is the Kolmogorov scale $L_{K}$ used in representing the lower bound of eddy size where viscosity damps out eddies. A statistical representation of the scale of turbulence is obtained from $L_{t}$. If one assumes an entrainment model of solid-body rotation, the largest turbulence scale is $2 L_{t}$; one may then define an overturning scale $L_{T}=2 L_{t}$. The buoyancy scale $L_{b}$ is similar to the Ozmidov scale, but includes possible effects of waves through the use of the fluctuating vertical velocity $w^{\prime}$. For this reason, $L_{R}$ is more representative of turbulence in stably-stratified conditions.

Results of experiments in the UCSD facility have provided a quantitative look at effects of stratification and shear on length scale behavior. As stratification increases ( $N$ becomes larger), $L_{R}$ is reduced as seen in Figure $2.21 \mathrm{a}$; in contrast, $L_{K}$ is affected very little by the the amount of stratification (see Figure 2.21b). The response of $L_{T}$ to buoyancy effects is a departure from an initial increase downstream of the grid. The greater the stratification, the greater and the earlier is the departure, as shown in Figure 2.21c. A comparison of Figures 2.21a and c suggests that $L_{T}$ grows while $L_{R}$ 
decreases until some critical value of $L_{R}$ is reached. Stillinger, et al. (1983) identified the following range of turbulent scales (L) for grid-generated turbulence in stratified flow (Stillinger, et al., 1983): $15.4 L_{K}<L<1.4 L_{R}$. The upper bound of $1.4 L_{R}$ represents the onset of buoyancy effects, while the lower bound of $15.4 L_{K}$ is where turbulence is extinguished.

From their work, Stillinger, et al. (1983) compiled a map showing the evolution of homogeneous turbulence in stably-stratified flow with no shear (see Figure 2.22). This pictorial model of length-scale behavior provides a quantitative framework for understanding the behavior of length scales as turbulence decays into internal waves. Just downstream of the turbulence-generating grid with a mesh size $M$, the largest turbulence scale $L_{T}$ is much smaller than $L_{R}$, the scale at which buoyancy effects become important. As the flows evolves downstream, both $L_{T}$ and $L_{K}$ grow as in unstratified conditions, defining an envelope or region of active, unaffected turbulence. As $L_{T}$ approaches $L_{R}$, the largest eddies become restricted in their overturning motion by having to work against buoyancy, while smaller eddies remain unrestricted. In the further evolution of the flow downstream, the turbulence collapses very rapidly, within about two-thirds of a large-eddy turnover time, as smaller scale overturning motion is suppressed (Van Atta, et al., 1984). Finally all that remains of the eddies are wave-like oscillations, after the turbulence has been extinguished by a combination of buoyancy and viscosity.

The presence of both stratification and shear in a stably-stratified flow can alter the above picture if stratification is not too strong. Figure 2.23 shows information for the unsheared case, given previously in Figure 2.21c, along with data for the sheared case.

When stratification is strong enough $\left(R_{i}>0.25\right)$, the behavior of the overturning scale for a given level of stratification $(N)$ is similar for both sheared and unsheared conditions (except that the sheared case behaves as if $N$ were slightly smaller). When stratification is weak $\left(R_{i}<0.25\right)$, the overturning scale behaves quite differently in the sheared case, reflecting a continued growth of turbulence downstream. In the unsheared case, the onset of buoyancy effects was seen in the decrease of $L_{T}$ at some point such that the ratio $L_{T} / L_{R}$ approached a constant, as shown by the solid line in Figure 2.24. In the sheared case with $R_{i}>0.25$, the length scale ratio approached 
a similar constant. When $R_{i}<0.25$, the shear provides enough turbulent energy so that both length scales grow. Their ratio approaches a much lower constant than for the unsheared case, as LR grows faster than $L_{T}$ in the weaker stratification (Rohr, et al., 1987).

The distribution of kinetic energy across a broad range of scales is portrayed through the use of spectra, as typified by Figure 2.7 in the previous subsection. At the upper (or high frequency) end of the spectrum, local isotropy is observed (Kaimal et al., 1972). As the amount of stratification increases, the wave-number range over which local isotropy holds decreases; also, the inertial subrange (or $-5 / 3$ range) retreats to higher wave numbers (Van Atta, 1985). Stratification acts to deplete turbulent energy in the low wave number (low frequency) range of the spectrum. However, as turbulent eddies are suppressed by buoyancy, the result is often low-frequency waves that show up in the vertical velocity spectra (Stillinger, et al., 1983), as illustrated in Figure 2.25. The low-frequency end of the spectrum for buoyancy-dominated flows is best scaled by a buoyancy wave number, $k_{b}=2 \pi / L_{b}=2 \pi / L_{R}$, which is where the damping occurs. High-frequency portions of the spectrum are better scaled by the Kolmogorov wave number, $k_{K}=2 \pi / L_{K}$, regardless of stratification conditions.

Early wind tunnel experiments by Webster (1964) showed a marked increase in the anisotropy of turbulence as stratification increased. Figure 2.26 gives evidence of this as the ratio of $\overline{w^{\prime 2}} / \overline{u^{\prime 2}}$ drops quickly from 0.8 to 0.4 as $R_{i}$ increases from 0 to 0.4 . Profiles of turbulence-intensity components, measured by Ogawa, et al. (1985), showed that the vertical component of turbulence decreases immediately in response to stratification effects, while the horizontal component decreases only under stronger stratification (see Figure 2.27a and b). This suggests that the buoyancy acts initially to suppress the vertical overturning of turbulent eddies, leading to "flatter" turbulent motion. Decaying turbulence in stratified flow also demonstrates increased anisotropy. The vertical turbulence $\left(\overline{w^{\prime 2}}\right)$ decays with downstream distance $(x)$ according to $\overline{w^{2}} \sim$ $x^{-1.4}$, while in the horizontal $\overline{u^{\prime 2}} \sim x^{-1}$ (Itsweire, et al., 1986). The faster decay rate for $\overline{w^{\prime 2}}$ seemed to be independent of the amount of stratification as long as the flow is buoyancy dominated. The decay rate for $\overline{u^{\prime 2}}$, on the other hand, did not appear to be affected by buoyancy. This result is not consistent with the findings of Ogawa, et al. (1985), but may be due to differences in experimental conditions, since Ogawa, 
et al. used a wind tunnel while the study of Itsweire, et al. was done in a salt-water channel.

The typical cascade of kinetic energy from generation at large scales to dissipation at the smallest scales is altered by the presence of sufficiently stable stratification. Some of the kinetic energy can be transferred back upscale to internal waves, rather than downscale to continue the cascade. This coexistence of waves and turbulence is most evident in the vertical velocity. As mentioned previously, use of $w^{\prime}$ in the definition of $L_{b}$ makes this length scale particularly sensitive to wave activity. The evolution of $L_{b}$, as seen in Figure 2.28, highlights the appearance of wave activity when turbulence drops off rapidly, as evidenced by $L_{R}$, while $L_{b}$ oscillates around a constant value. Values for $L_{b}$ and $L_{R}$ for various stratifications are related rather well for $L_{R}>1 \mathrm{~cm}$, with a good fit between scales of $L_{R}=1.5 L_{b}^{1.5}$ (Itsweire, et al., 1986).

Wave activity can be distinguished not only in the relative behavior of length scales, but also more directly in the evolution of vertical velocity fluctuations. When the turbulent component is separated from the total vertical velocity variance for stratified flow, the turbulent component was found to decay faster than for unstratified flow, while the total variance remained the same or was slightly larger than for the unstratified case (see Figure 2.29). This result suggests that stratification increases the decay of the vertical component of TKE, but that some of the lost TKE is merely transferred to potential energy (as IGW reflected in the vertical velocity variance) and not lost from the total system any faster than in the unstratified case.

This transfer of KE to $\mathrm{PE}$ is a critical element in the energetics of stratified flow. It is the buoyancy flux $\overline{\rho w}$ that causes the transfer. In the TKE equation for the SBL buoyancy flux acts as a sink of KE, while in the equation for fluctuating PE $\left(\overline{\rho^{2}}\right)$ buoyancy flux occurs in the production term (Van Atta, 1985). Late in the evolution of stratified flows, when all that remains is an internal wave field, $\overline{\rho w}$ vanishes. In strong stratification, Itsweire, et al. (1986) found that the evolution of $\overline{\rho w}$ involves an overshoot to negative values and subsequent counter-gradient flux as the flow restratifies (see Figure 2.30). Periods of counter-gradient flux under strong stratification were also observed by Komori, et al. (1983). In numerical simulations of even stronger stratification, Riley et al. (1981) found that the positive/negative oscillations (or ringing) of $\overline{\rho w}$ continued for several cycles. 
Comparison of the evolution of KE and PE, as seen in Figure 2.31, shows that relatively more $\mathrm{PE}$ is gained under stronger stratification. The fraction of KE that is transferred to PE, instead of being lost to dissipation, can be represented by a flux Richardson number $R_{f}$, i.e. the ratio of KE loss due to buoyancy over the total loss of KE (buoyancy and dissipation). Values for $R_{f}$ for increasing stratification, represented by the gradient Richardson number $R_{i}$, show an initial increase in the fraction of $\mathrm{KE}$ converted to $\mathrm{PE}$; this fraction is approximately $R_{f}=0.13 R_{i}$. At stratification greater than about $R_{i}=1.25, R_{f}$ begins to decrease, leaving a maximum transfer of KE to PE of about 15-20\%. The maximum transfer (or mixing efficiency) is thought to occur at the point where the where the turbulence scale approaches $L_{R}$, i.e. at the strongest stratification that still allows overturning of the largest eddies (Rohr, et al., 1984).

When stable stratification is strong enough, the turbulence can become intermittent, rather than uniformly small or nonexistent. In such circumstances, statistics of the active turbulence, such as dissipation rate, are usually underestimated because turbulent and non-turbulent periods are included. Correction factors for intermittency (in time or space) can be developed based on the distribution of statistics. An example of this is a correction factor of $e^{3 \sigma^{2} / 2}$ for dissipation, where $\sigma$ is the geometric standard deviation of the lognormal distribution of calculated dissipation rates (Gibson, 1981). Another approach is conditional sampling where statistics are calculated on a subset of data extracted from the total possible data set based on the value of an indicator parameter (Khalsa, 1980; Antonia, et al., 1983). For example, to obtain statistics representative of active turbulence in a stably-stratified flow, one could select data for analysis based on the condition that the indicator parameter $L_{T}$ be less than $1.4 L_{R}$.

Piccirillo and Van Atta (1997) investigated turbulence evolution in a homogeneous stably stratified shear flow using a thermally-stratified wind tunnel. This is attractive since is more similar to the atmospheric case than using salt water. They varied the turbulence-generating grids to vary the Reynolds number; that is, as the grid size increases, the Reynolds number increases. They found that the critical Richardson number, which is the demarcation between growth or decay of turbulence, decreased as Reynolds number increased. 


\subsubsection{Limitations of laboratory studies}

Although the laboratory provides a controlled environment for studying turbulence, there are artifacts associated with experimental conditions that must be considered. For cases of stratified fluids in tanks, the way in which grid turbulence is introduced can have an effect on the results. If a grid is towed or dropped vertically (i.e. as in Dickey and Mellor, 1980), natural internal wave modes are excited that can contaminate measurements of velocity fluctuations; in contrast, for a horizontally towed grid in a long tank, natural modes are at very low frequency and have negligible affect on the turbulence (Britter, et al., 1983). In stratified flows at the UCSD facility, Itsweire et al. (1986) found that the grid-mesh size M (grid spacing) had an effect on the initial evolution of turbulence. Large-scale initial turbulence generated by the large-mesh grid $(\mathrm{M}=3.8 \mathrm{~cm})$ overwhelmed waves created by the grid; results were similar to the unstratified case. For the small-mesh grid $(\mathrm{M}=1.9 \mathrm{~cm})$, however, grid-generated waves coexisted with the turbulence. Also, stratification effects were limited to the evolution of $\overline{w^{\prime 2}}$ in the large-mesh experiments, while effects were seen in both vertical and horizontal velocity fluctuations for the small-mesh experiments.

Apparatus dimensions can have an effect as well. Webster (1964) concluded that his measurements were in a developing flow and that his wind tunnel would have to be 2 or 3 times longer in order to achieve the desired steady-state conditions. In stratified wind tunnel flows, Reynolds-number effects can become more important as static stability increases (Arya, 1975). It is very difficult to achieve buoyancydominated flows in the wind tunnel because of the large temperature gradients (the usual means for establishing stratification) needed to compete with the inertial and viscous forces present (Itsweire, et al., 1986).

It is difficult to apply the results of laboratory experiments, conducted in controlled conditions, to the complex conditions found in the PBL. Indeed the PBL does not usually have a well-defined outer edge, as do laboratory flows (Cermak and Arya, 1970). Laboratory conditions may allow study of selected processes at low Reynolds number. At high Reynolds numbers typical of atmospheric flows, there is a much wider range in the scales of turbulent motion; applying low Reynolds-number results to the atmosphere must be done therefore with caution (McEwan, 1983). Nevertheless, the findings of the above studies provide corroborative and often alternative 
understandings relative to the previous studies of atmospheric turbulence. 
Table 2.1: Length Scales Characteristic of Stably-Stratified Turbulent Flows (from Itsweire, et al., 1986).

\begin{tabular}{|l|l|l|l|}
\hline Lengthscale & Symbol & Definition & Physical description \\
\hline \hline Ozimodov scale & $L_{R}$ & $\left(\varepsilon / N^{3}\right)^{1 / 2}$ & $\begin{array}{l}\text { Scale at which } \\
\text { buoyancy forces equal } \\
\text { inertial forces }\end{array}$ \\
\hline Kolmogorov scale & $L_{K}$ & $\left(\nu^{3} / \varepsilon\right)^{1 / 4}$ & $\begin{array}{l}\text { Scale at which } \\
\text { viscous forces } \\
\text { equal inertial forces }\end{array}$ \\
\hline Turbulent scale & $L_{t}$ & $\rho^{\prime} /(\partial \bar{\rho} / \partial z)$ & $\begin{array}{l}\text { Typical vertical distance } \\
\text { travelled by fluid particles } \\
\text { before either returning } \\
\text { to their equilibrium mixing }\end{array}$ \\
\hline Buoyancy scale & $L_{b}$ & $w^{\prime} / N$ & $\begin{array}{l}\text { Vertical distance travelled } \\
\text { by a fluid particle in } \\
\text { converting all its } \\
\text { vertical kinetic energy } \\
\text { into potential energy }\end{array}$ \\
\hline Ellison scale & $L_{E}$ & $-\bar{\rho}^{2}$ & \\
& & $\begin{array}{l}\text { Maximum overturning length } \\
\text { scale for the existing } \\
\text { density stratification }\end{array}$ \\
\hline \hline
\end{tabular}




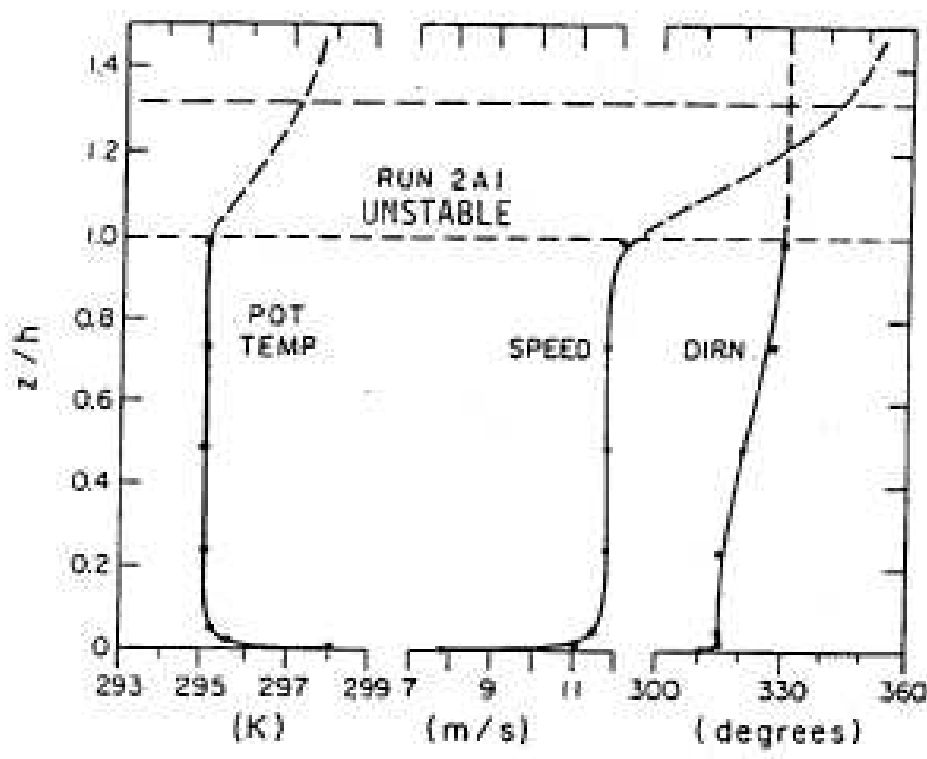

(a)

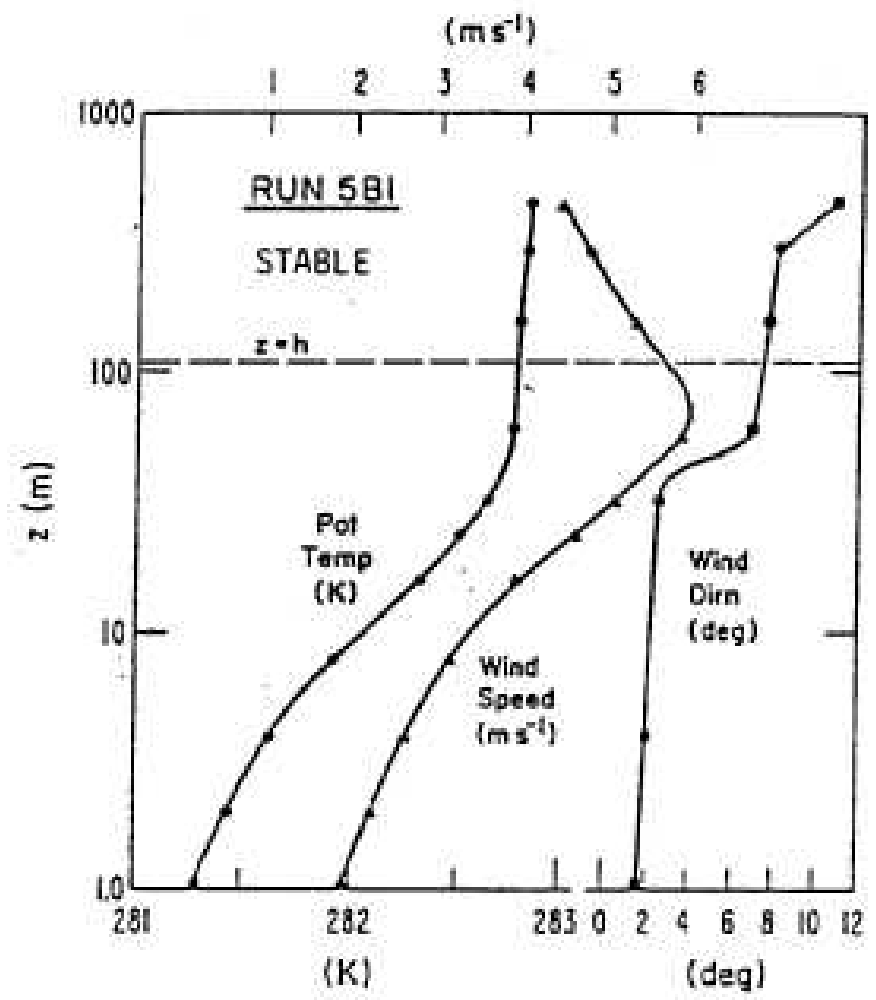

(b)

Figure 2.1: Typical mean profiles of wind speed, wind direction and potential temperature showing their relationship to boundary-layer depth, h, for (a) the CBL (after Kaimal, et al., 1976), and (b) the SBL (after Caughey, et al., 1979). 


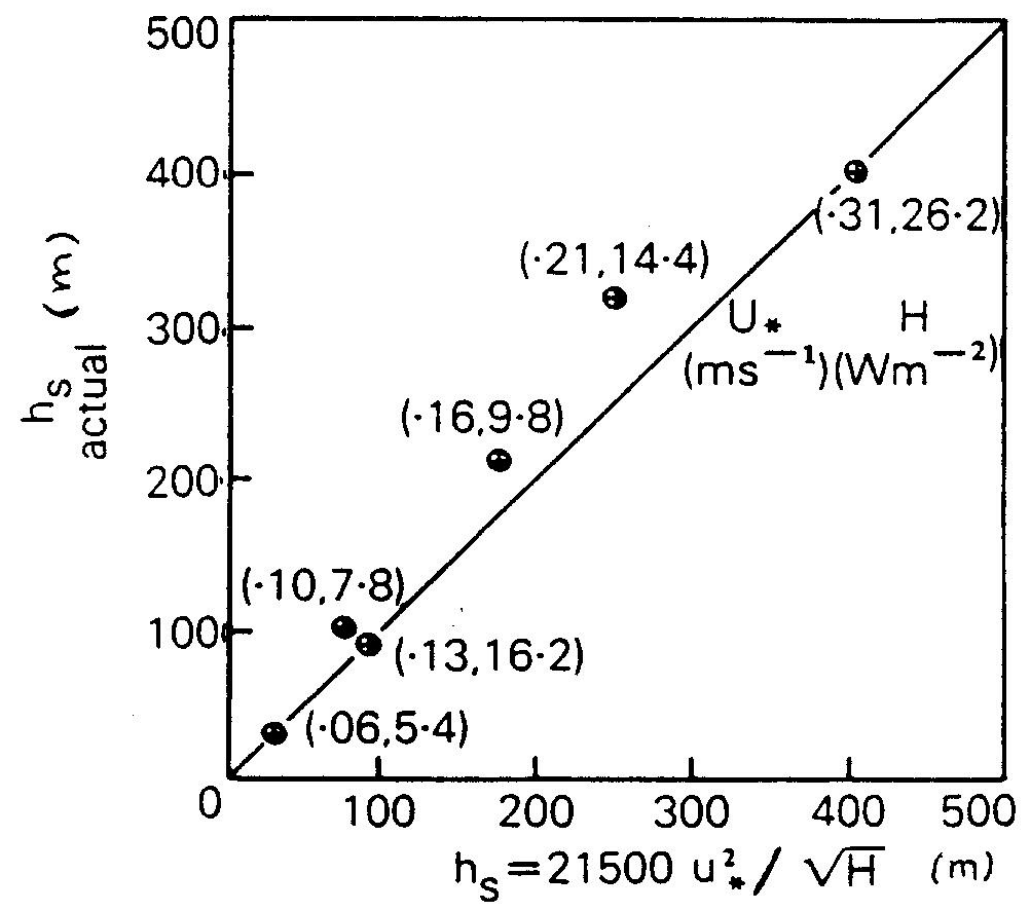

Figure 2.2: Comparison of data from the Minnesota Experiment to an estimate of the SBL depth, $h_{s}$, based on the friction velocity, $u_{*}$, and negative surface heat flux, $\mathrm{H}$. 


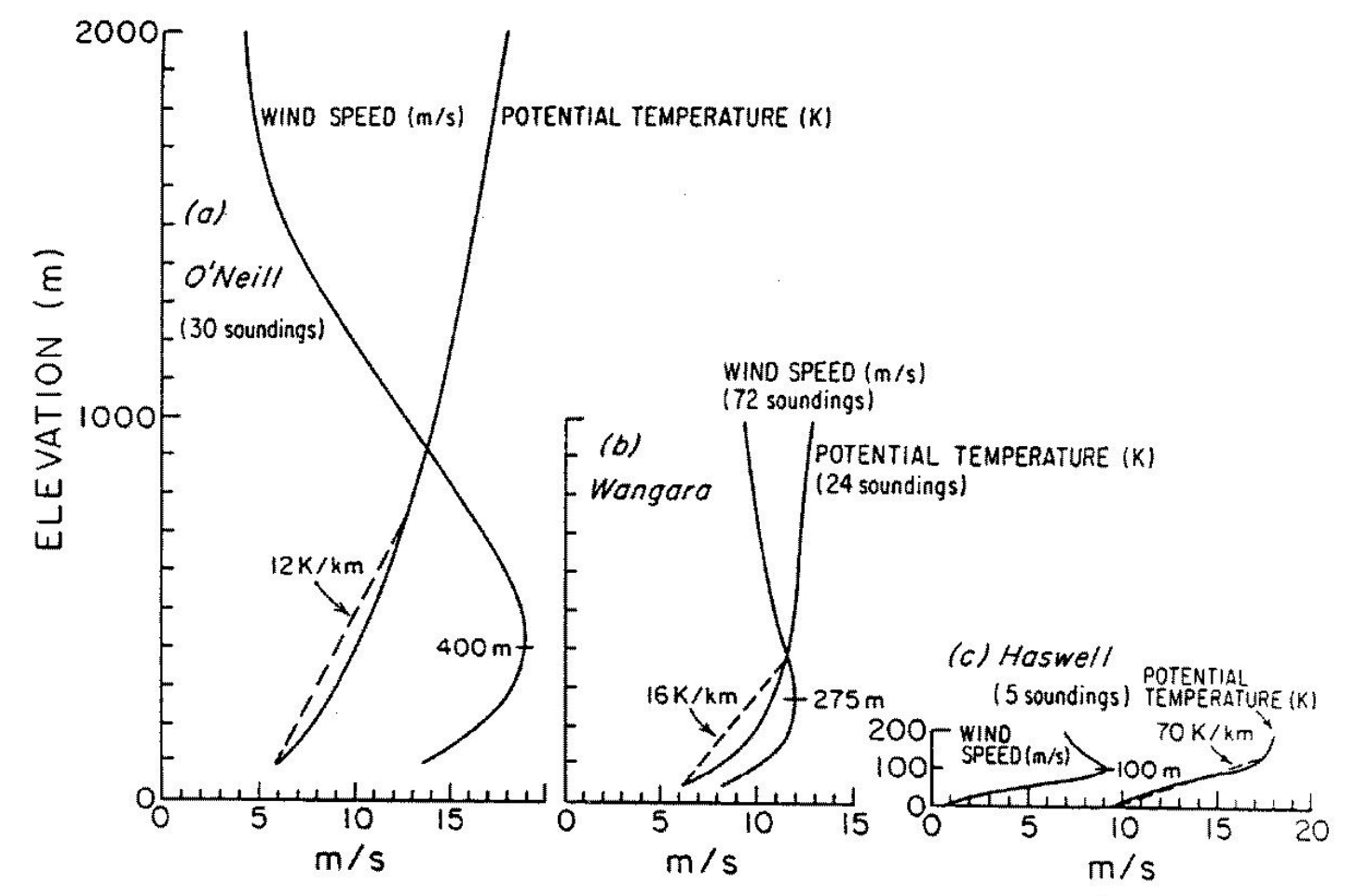

Figure 2.3: Mean profiles of the SBL as observed at O’Neill, Nebraska, Wangara, Australia, and Haswell Colorado. 


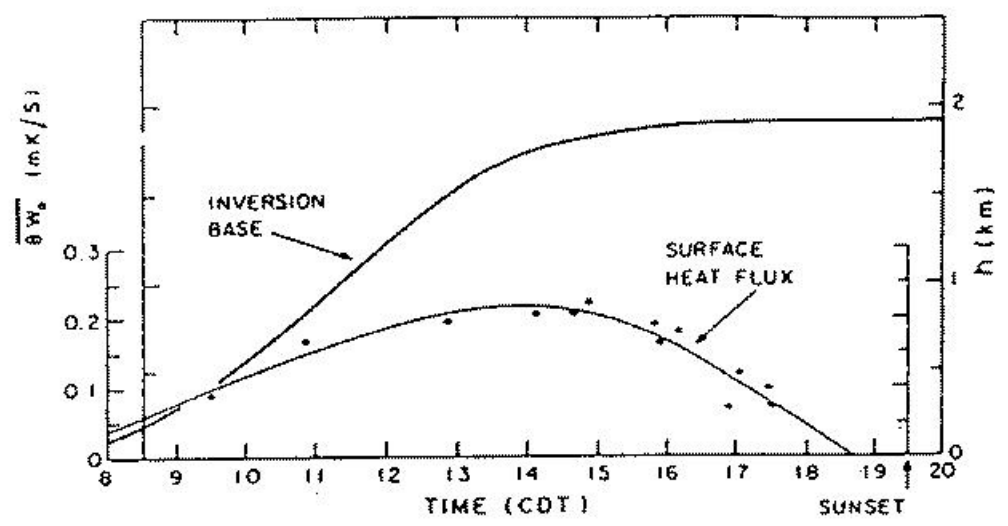

(a)

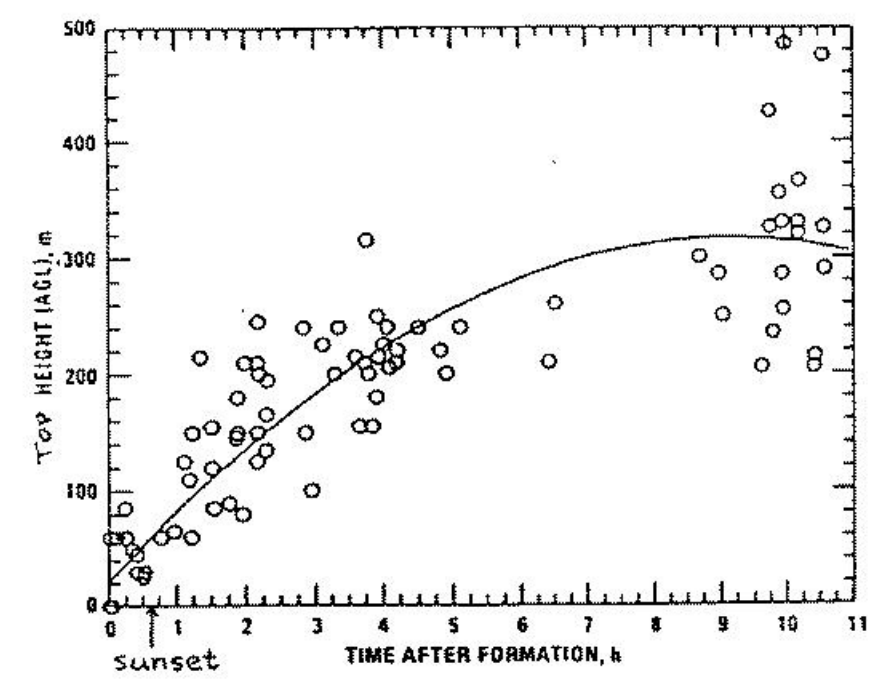

(b)

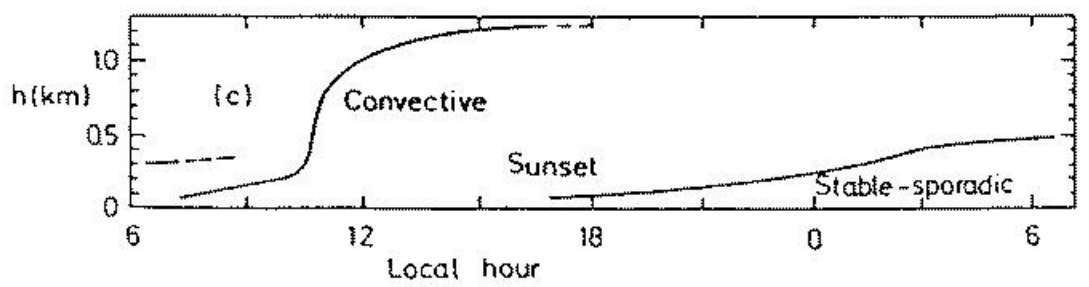

(c)

Figure 2.4: Diurnal trends for (a) the height of the temperature inversion capping the CBL as measured for a typical day in the Minnesota Experiment (Kaimal et al., 1976), (b) the inversion top at a rural site during 20 summer nights, and (c) the superposition of the CBL and SBL to illustrate the diurnal cycle. 


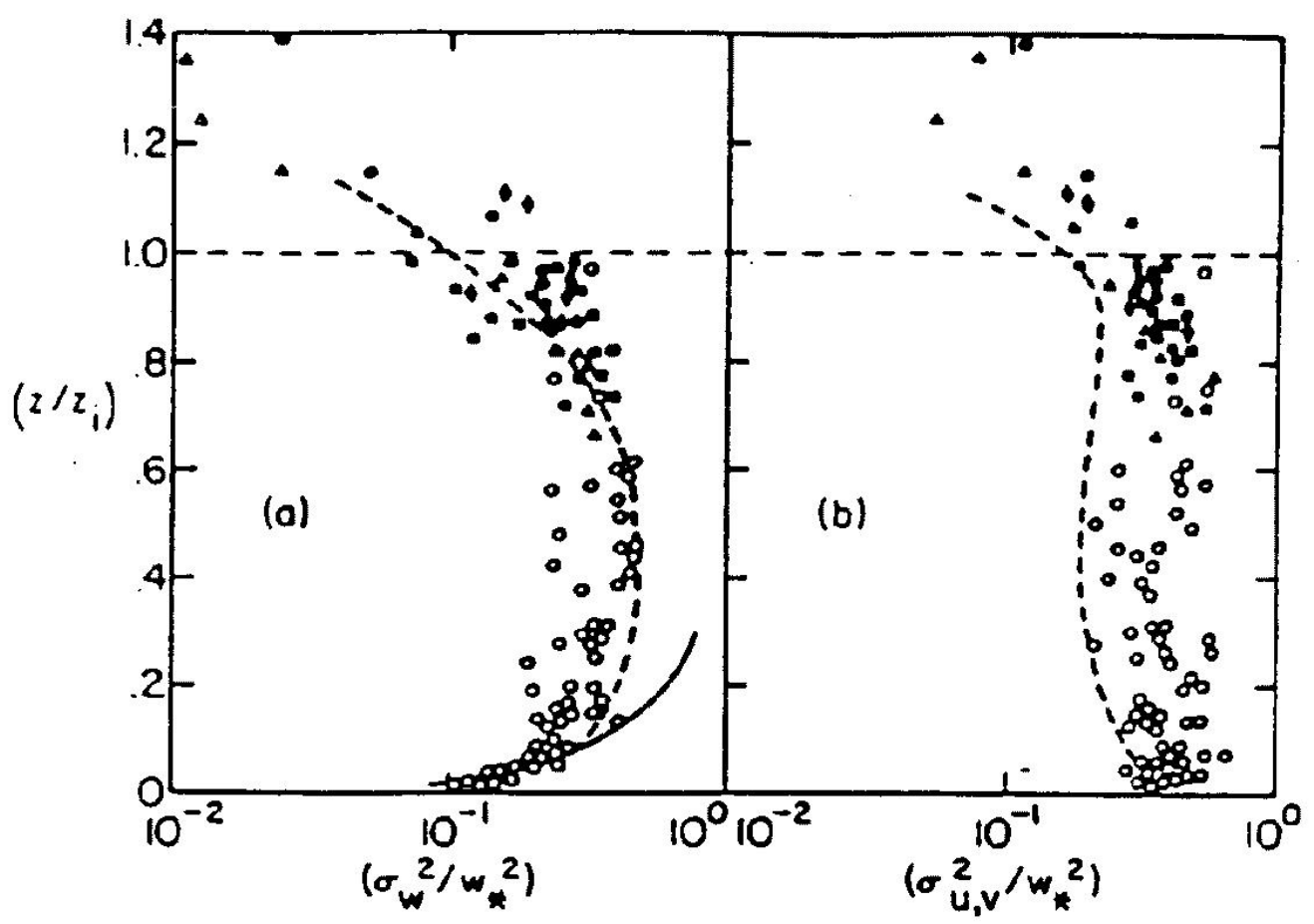

Figure 2.5: Vertical and horizontal velocity variances, normalized by the squared convective velocity scale, $w_{*}^{2}$; solid lines: free convection; dashed lines: laboratory data (Willis and Deardorff, 1974); open circles: Minnesota data; solid symbols: observations at Ashchurch, England (Caughey and Palmer, 1979).
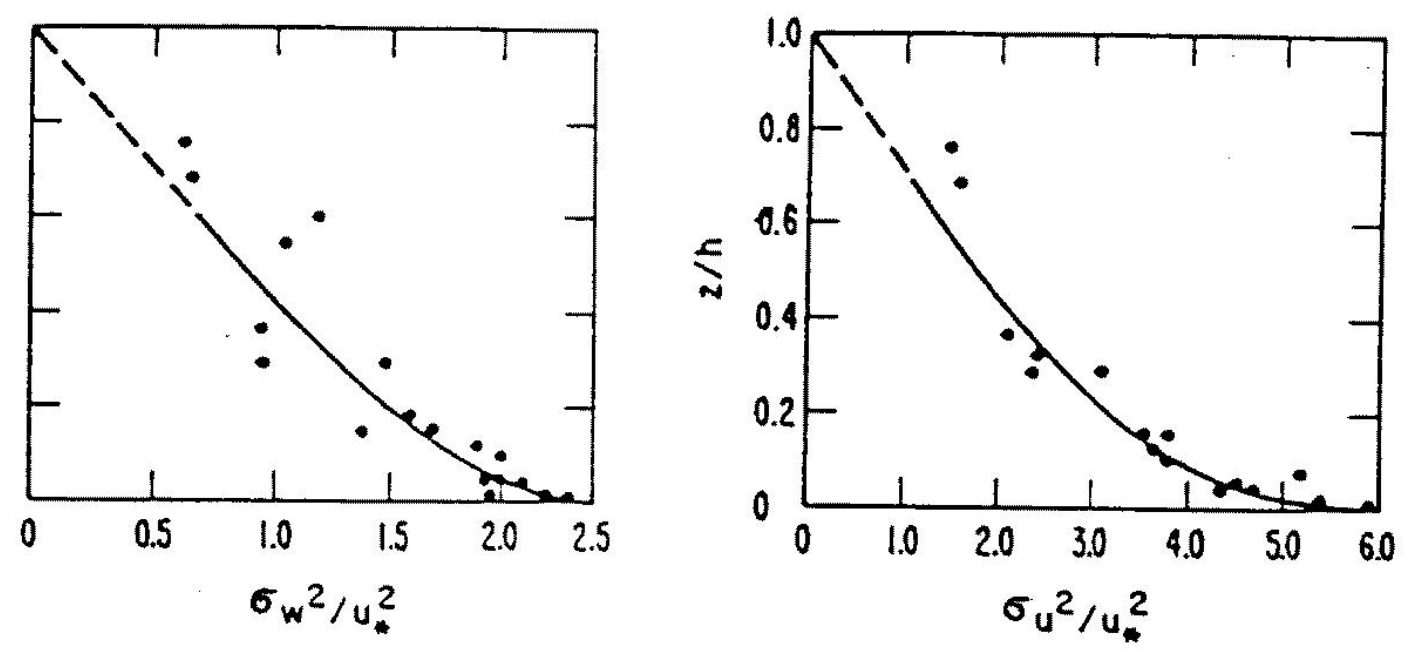

Figure 2.6: Vertical profiles of normalized velocity variances for the SBL (after Caughey, et al., 1979). 


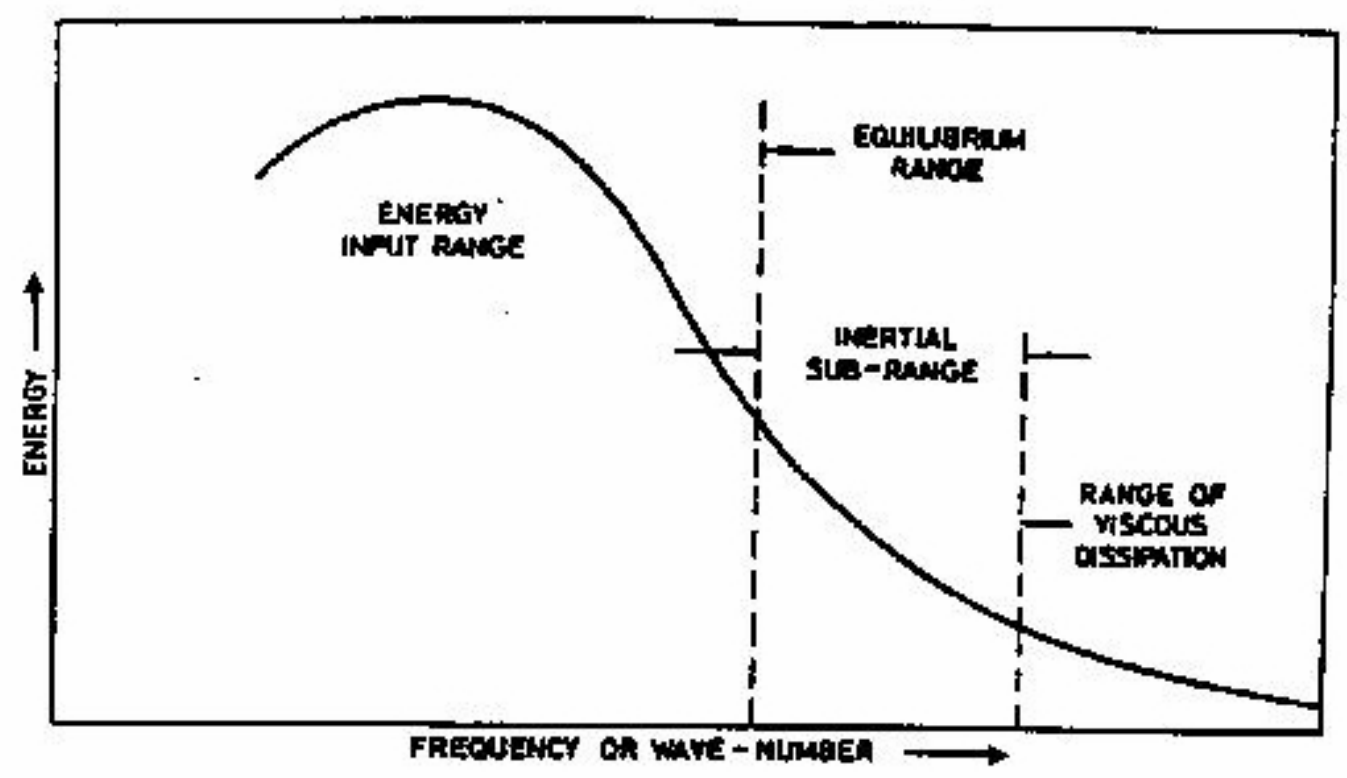

Figure 2.7: Schematic representation of the energy spectrum of turbulence.

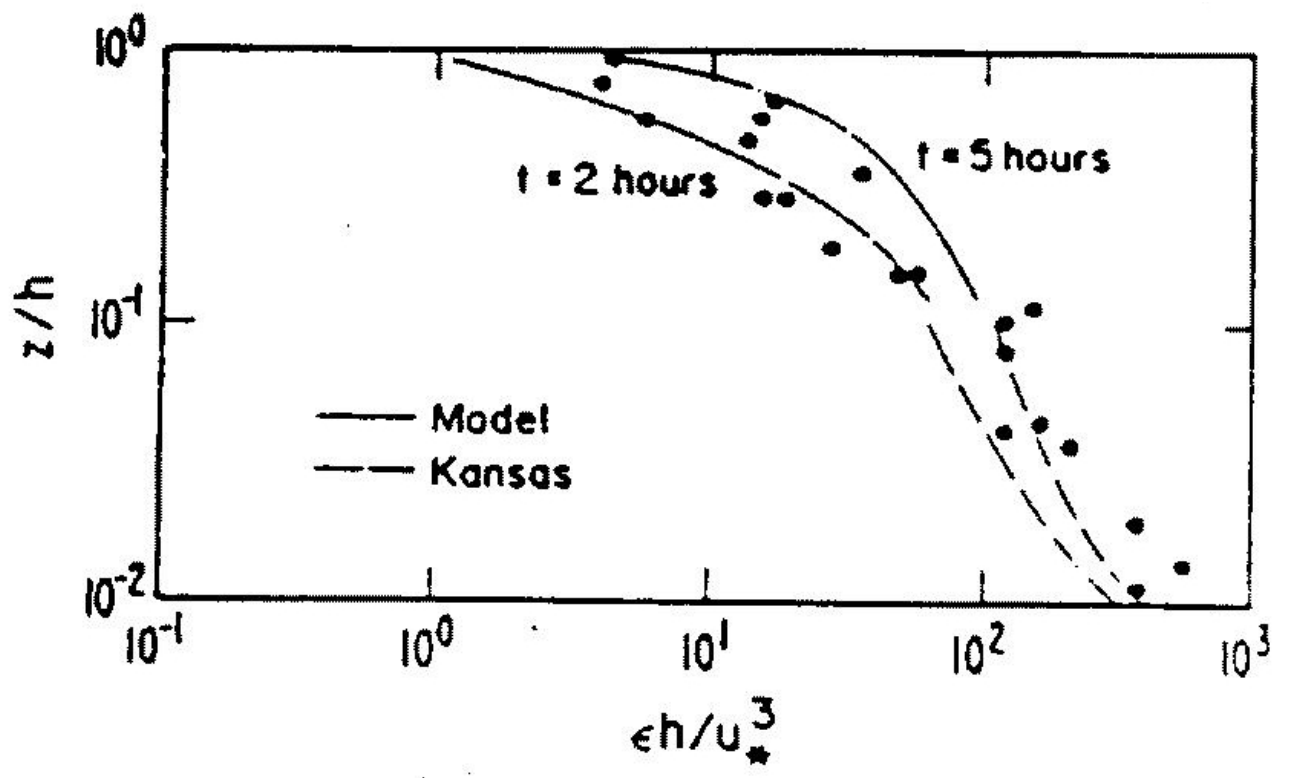

Figure 2.8: Vertical profiles of dissipation rate for the SBL (after Caughey, et al., 1979). 
(a)

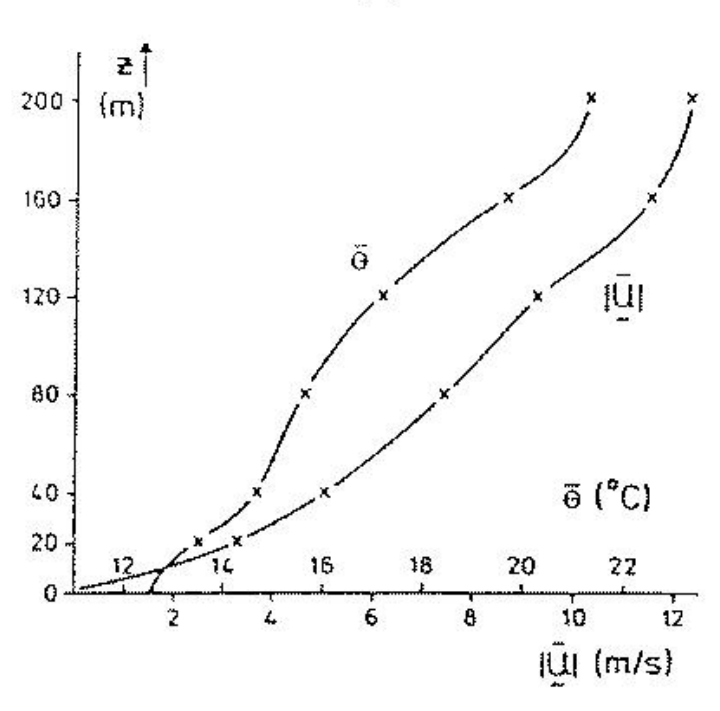

(b)

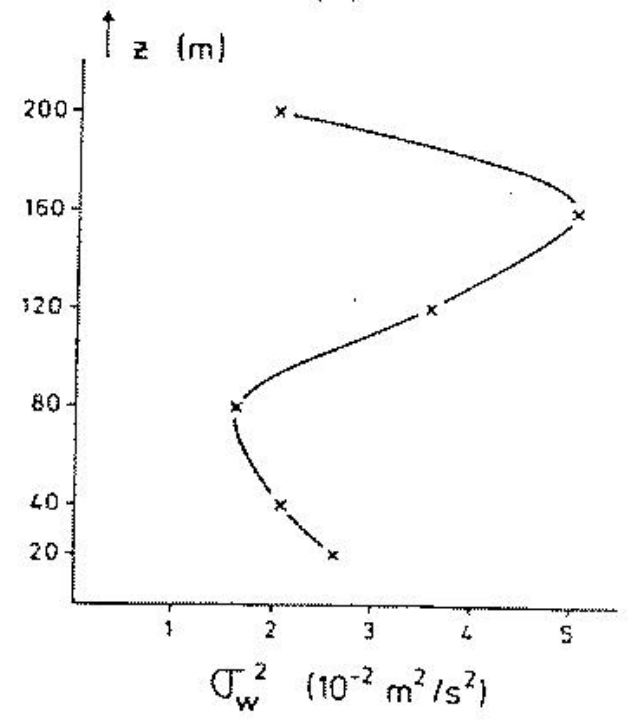

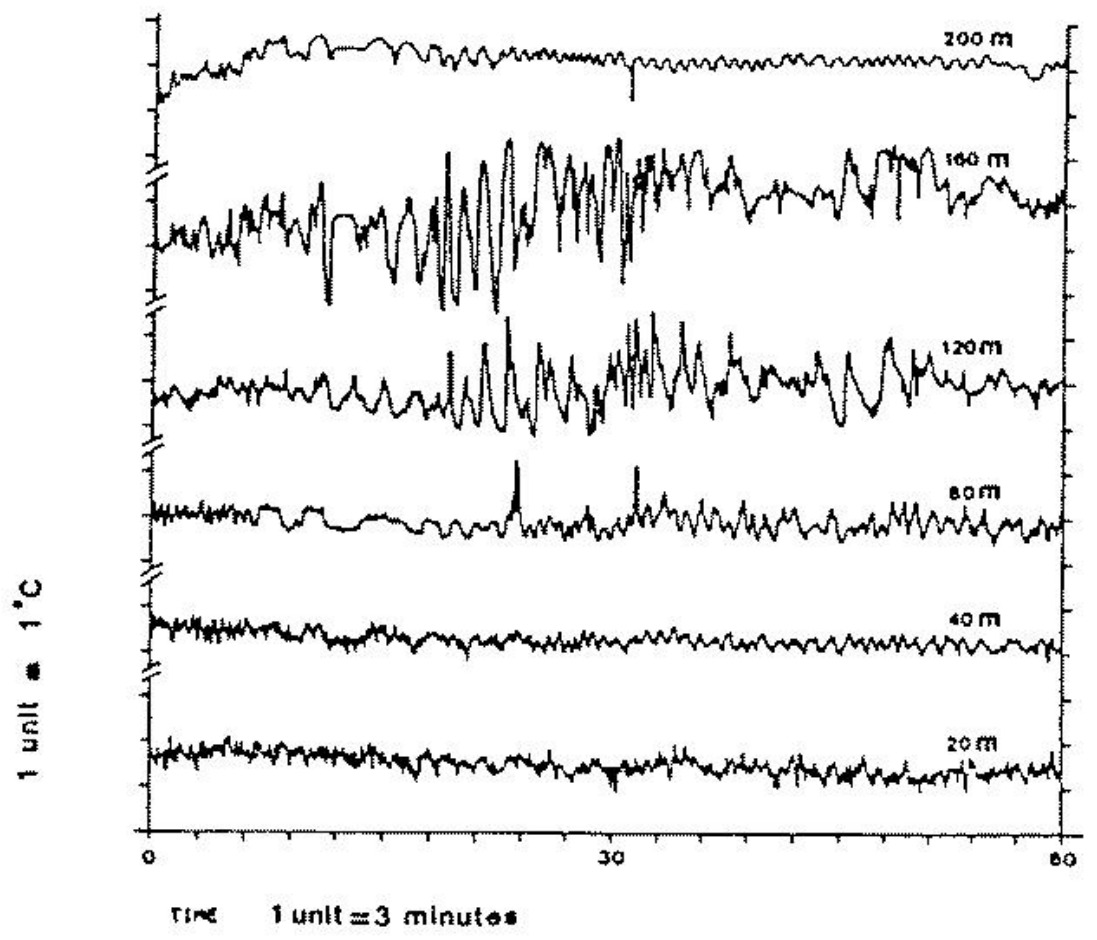

(c)

Figure 2.9: Measured SBL profiles from the Cabauw tower, 30 May 1978, 01-02 UTC, for (a) hourly mean wind speed $\mathrm{u}$ and temperature, (b) variance of vertical velocity, and (c) temperature traces at the six tower heights; the height of the SBL was about $180 \mathrm{~m}$ (after DeBass and Driedonks, 1985). 

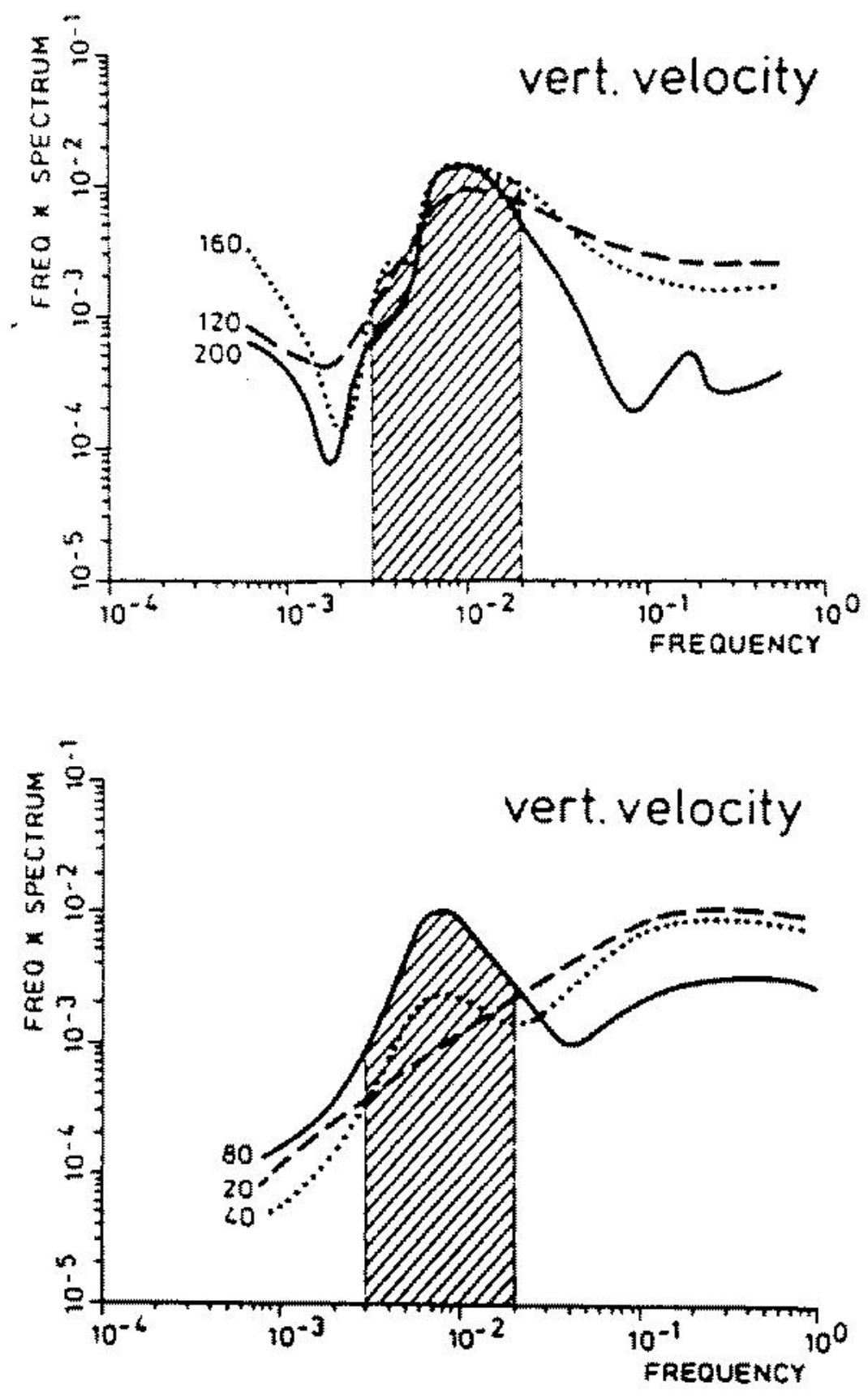

Figure 2.10: Variance spectra of vertical velocity and temperature from six heights (20 to $160 \mathrm{~m}$ ) on the Cabauw tower, 5/30/78, 01-02 UTC (after DeBass and Driedonks, 1985). 

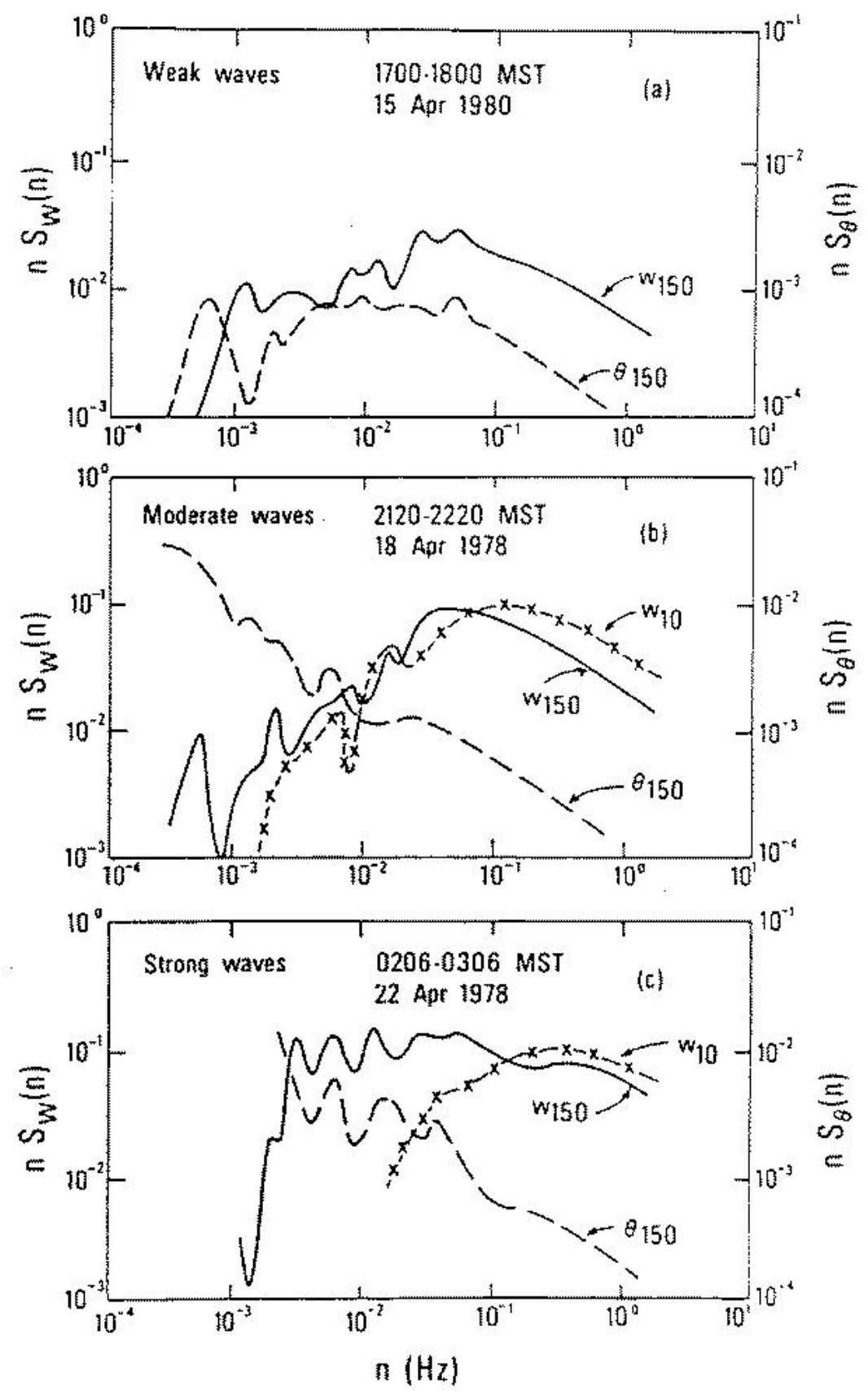

Figure 2.11: Spectra of vertical velocity and temperature for (a) weak, (b) moderate, and (c) strong wave cases observed at the 10- and 150-m heights on the BAO tower (after Hunt, et al. 1985). 


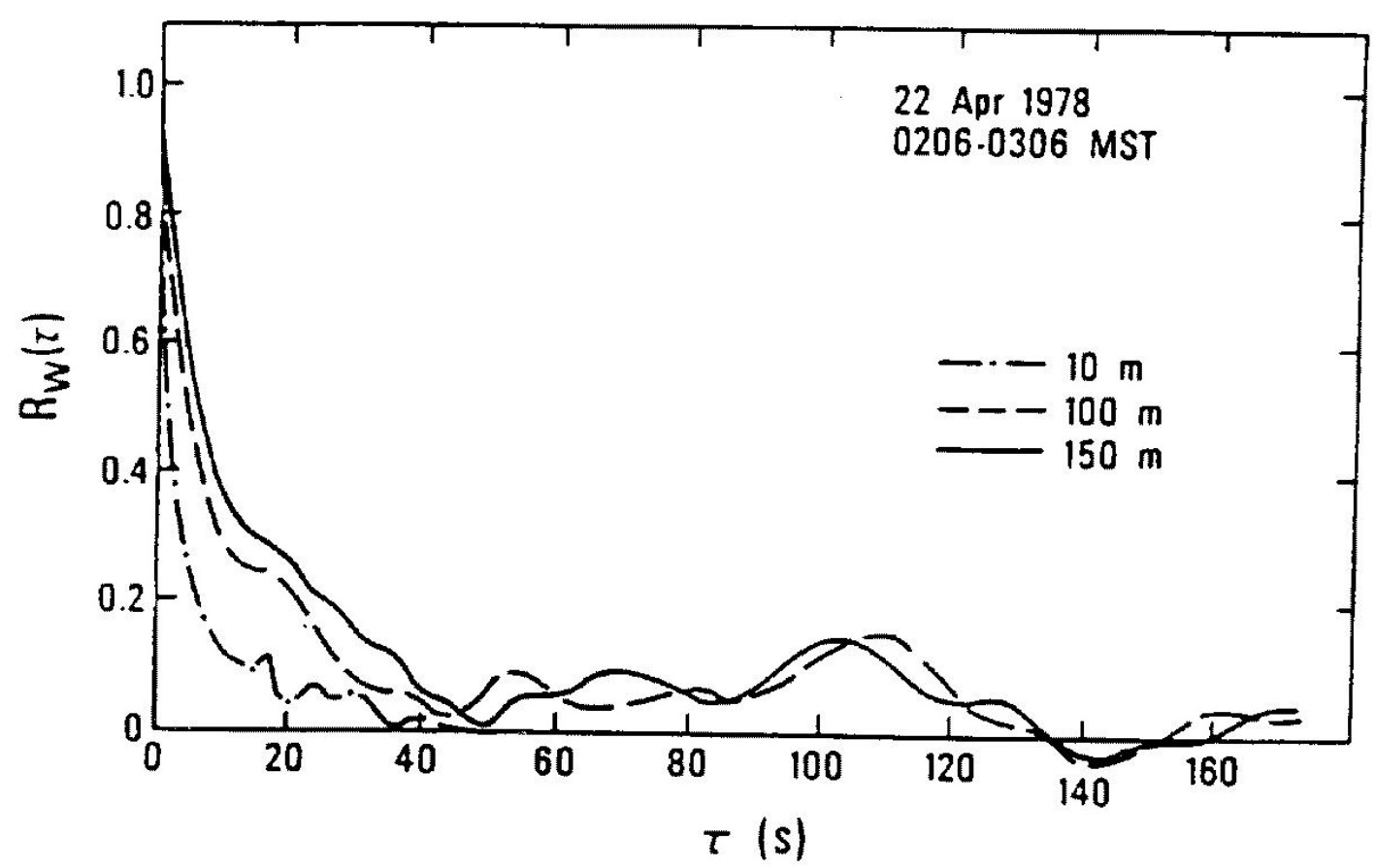

Figure 2.12: Autocorrelation of vertical velocity at three heights on the BAO tower (after Hunt, et al. 1985).

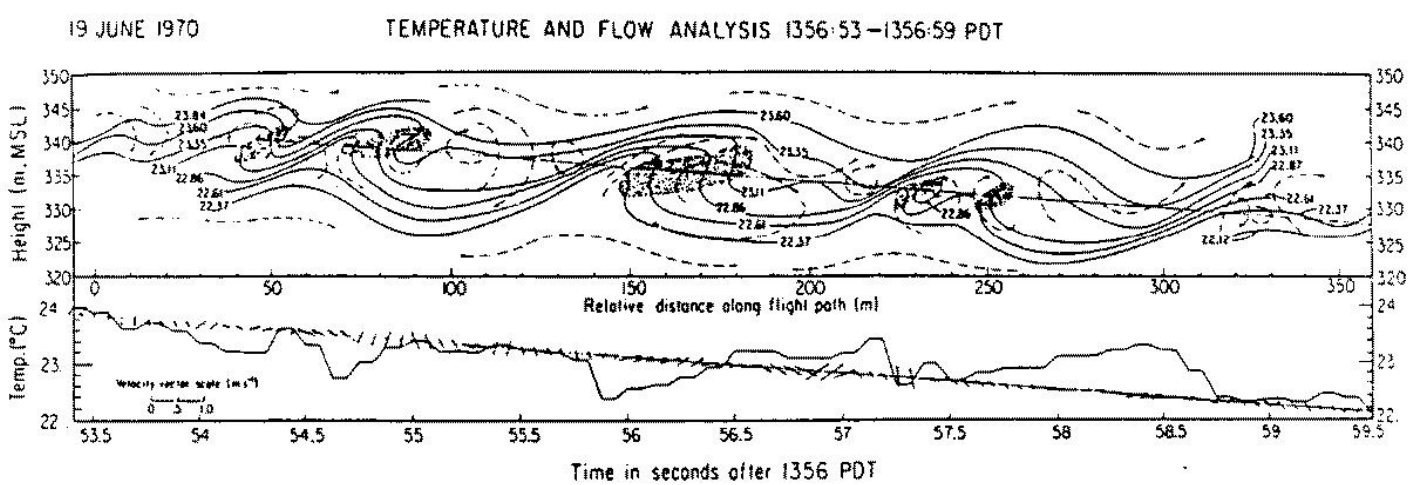

Figure 2.13: Temperature and vertical velocity records and wave analysis for an aircraft flight through an elevated inversion; thermally unstable regions due to overturning are shaded (after Metcalf and Atlas, 1973). 


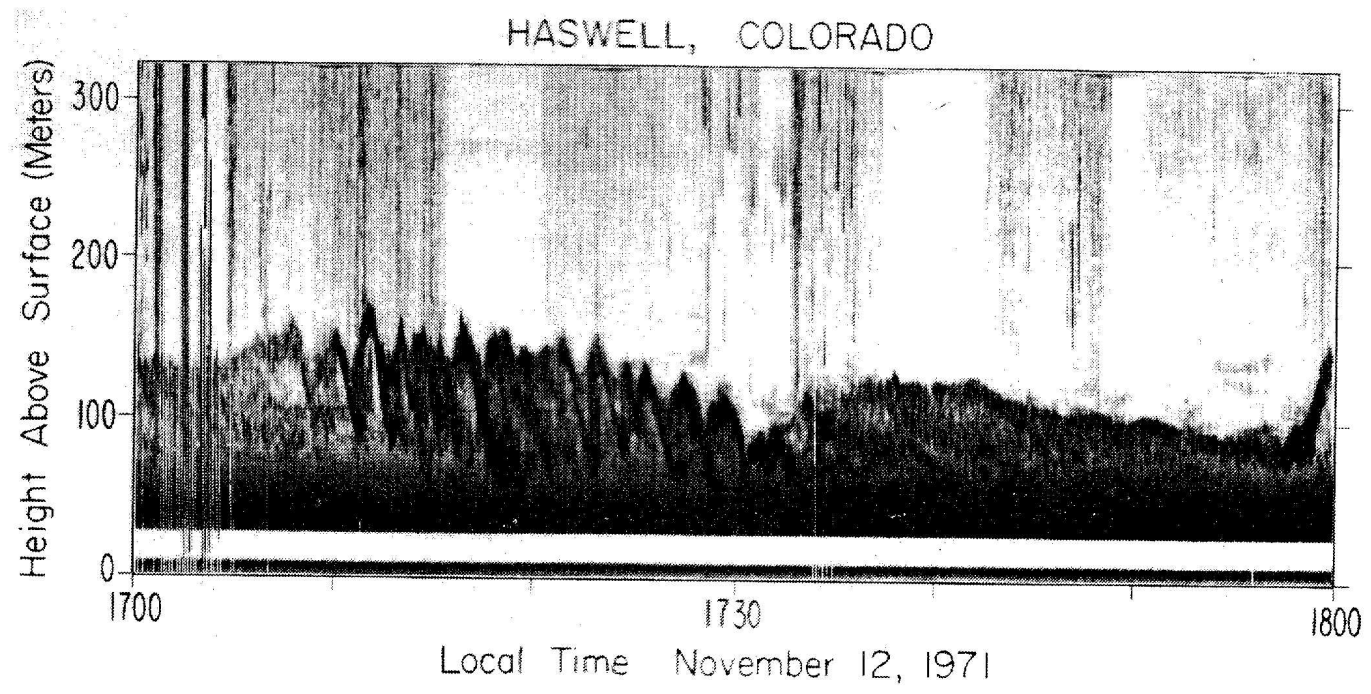

Figure 2.14: The acoustic echo-sounder facsimile record for 1700-1800, 12 Nov 1971, at Haswell, CO; dark areas of the plot represent height-time intervals of strong echo returns (after Hooke, et al. 1973). 

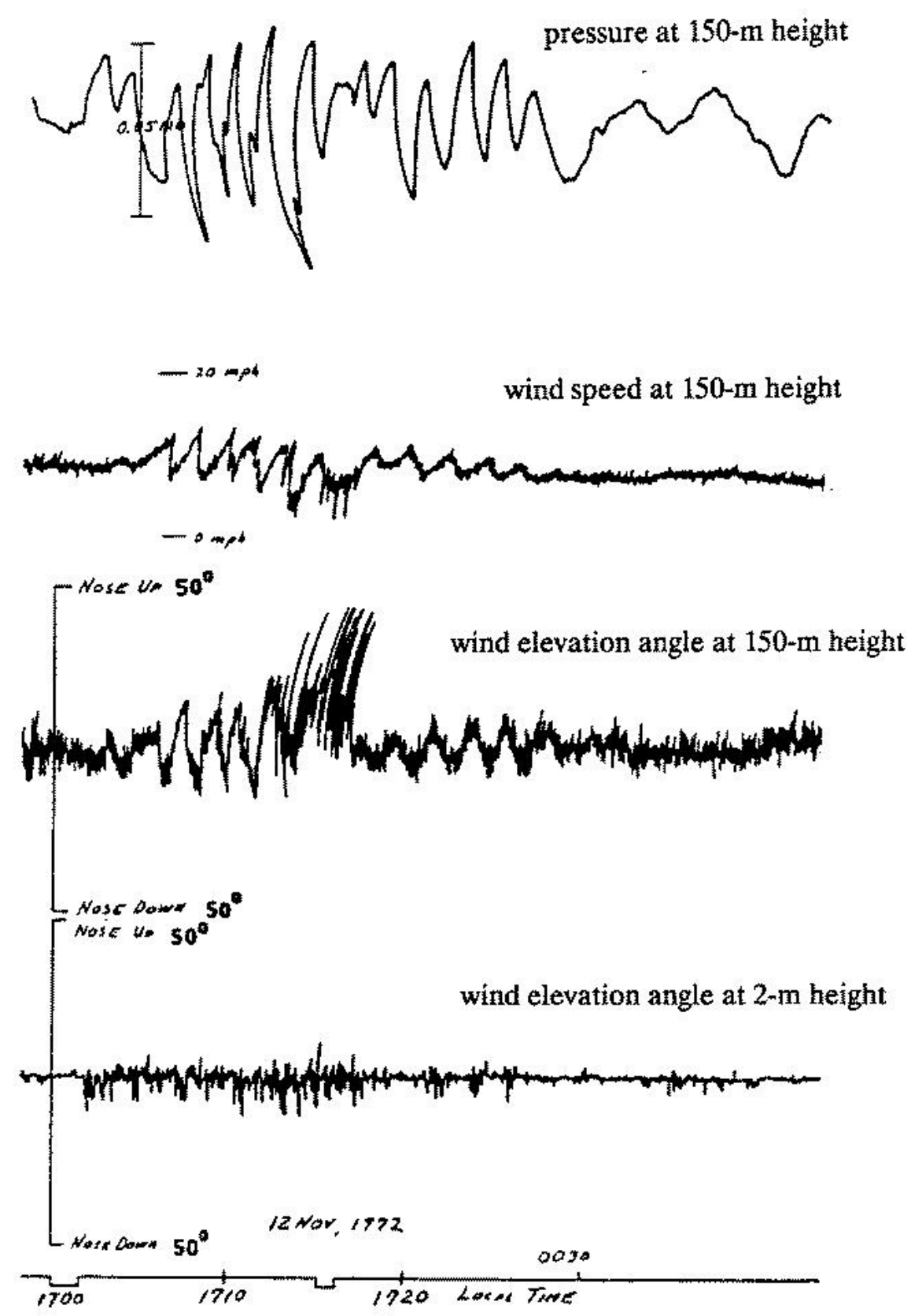

Figure 2.15: Raw microbarograph and anemometer records from the top of a $150-\mathrm{m}$ tower and near the surface for the wave-generation event shown in Figure 2.14 (after Hooke, et al., 1973). 


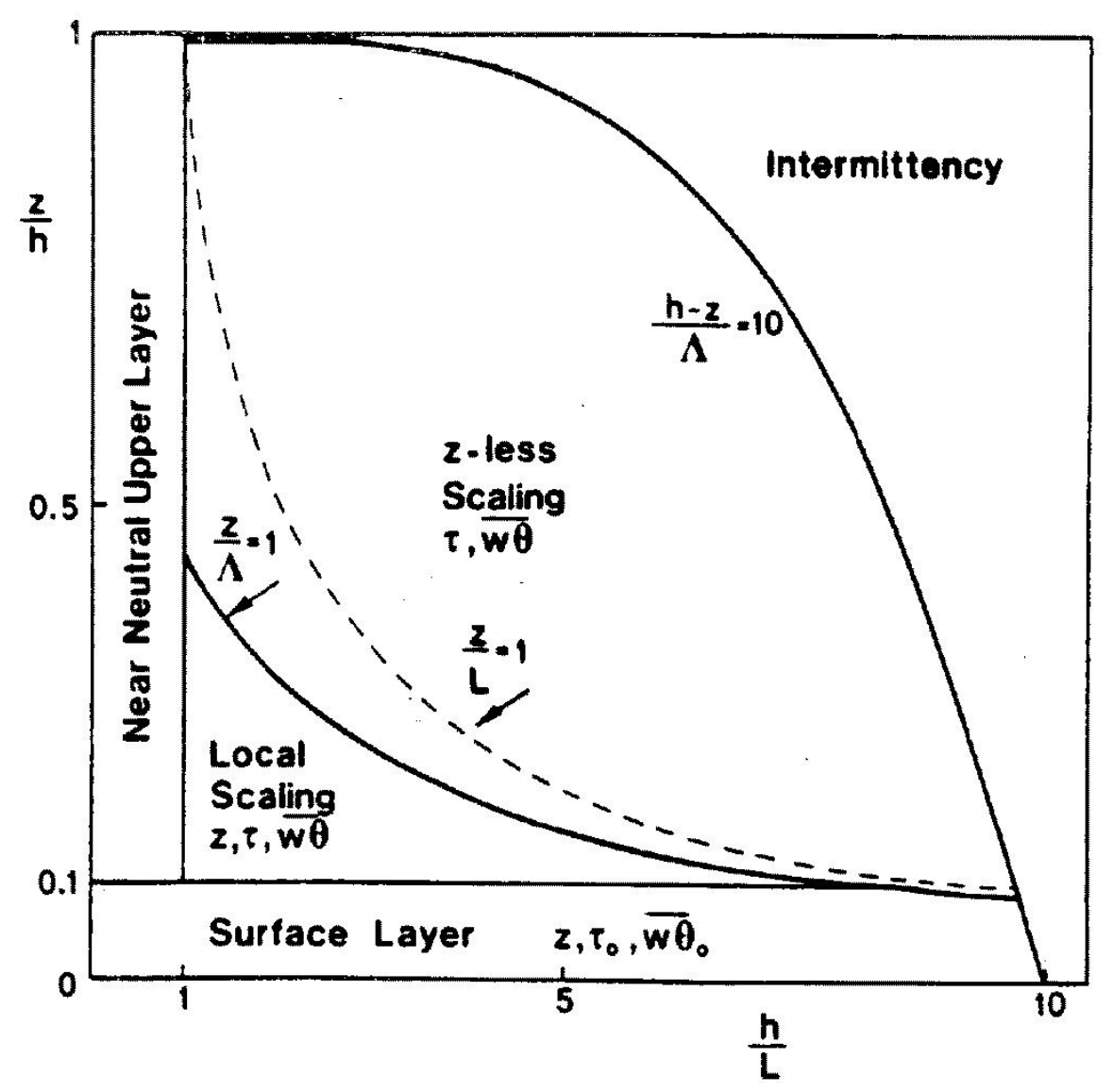

Figure 2.16: Scaling regions for the SBL (after Holtslag and Nieuwstadt, 1986) 

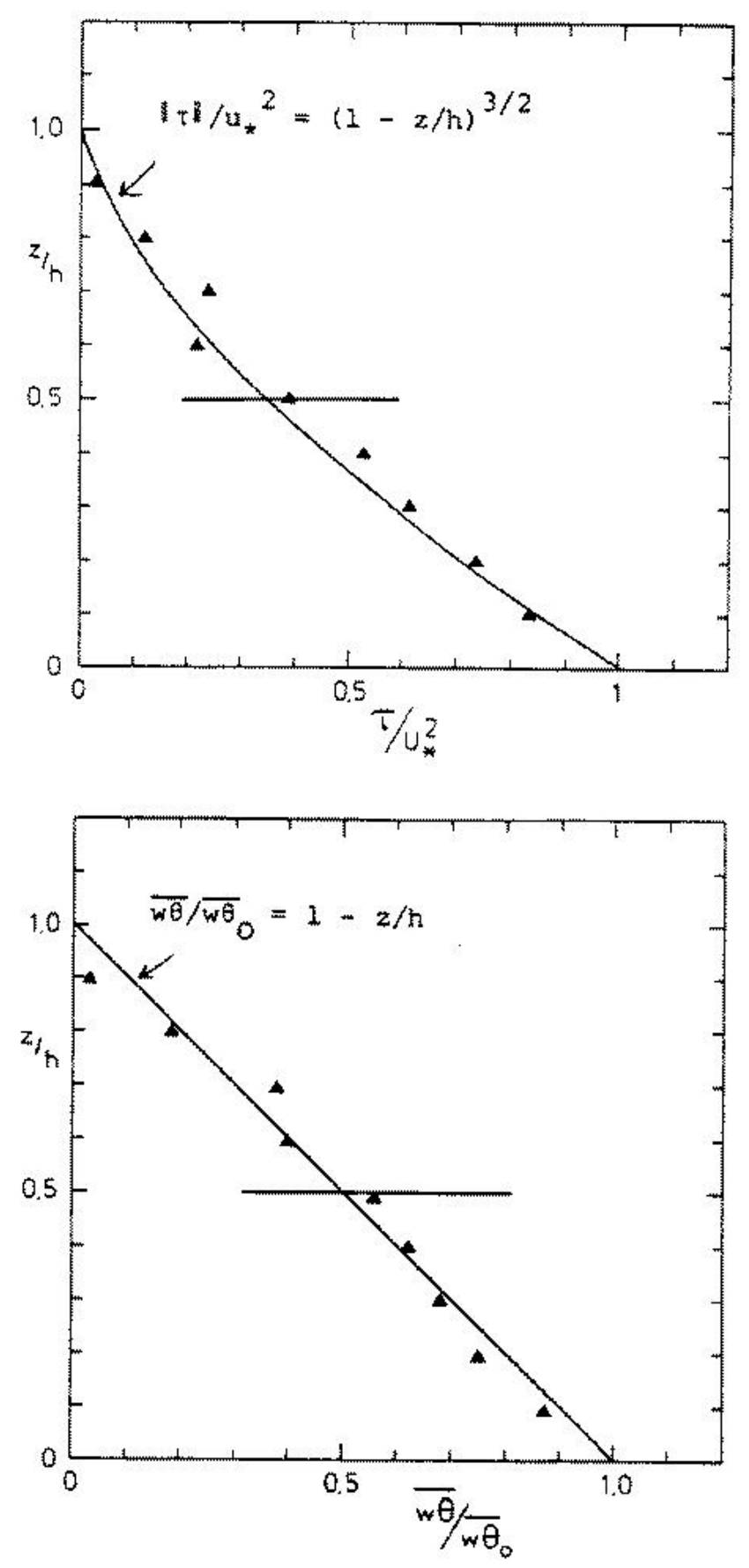

Figure 2.17: Profiles of (a) momentum flux and (b) temperature flux non-dimensionalized by respective surface values; height is normalized by the SBL height $\mathrm{h}$; solid triangles represent average values from experiments at the Cabauw tower, with variability indicated by horizontal solid line midway in the profile (after Nieuwstadt, 1985). 


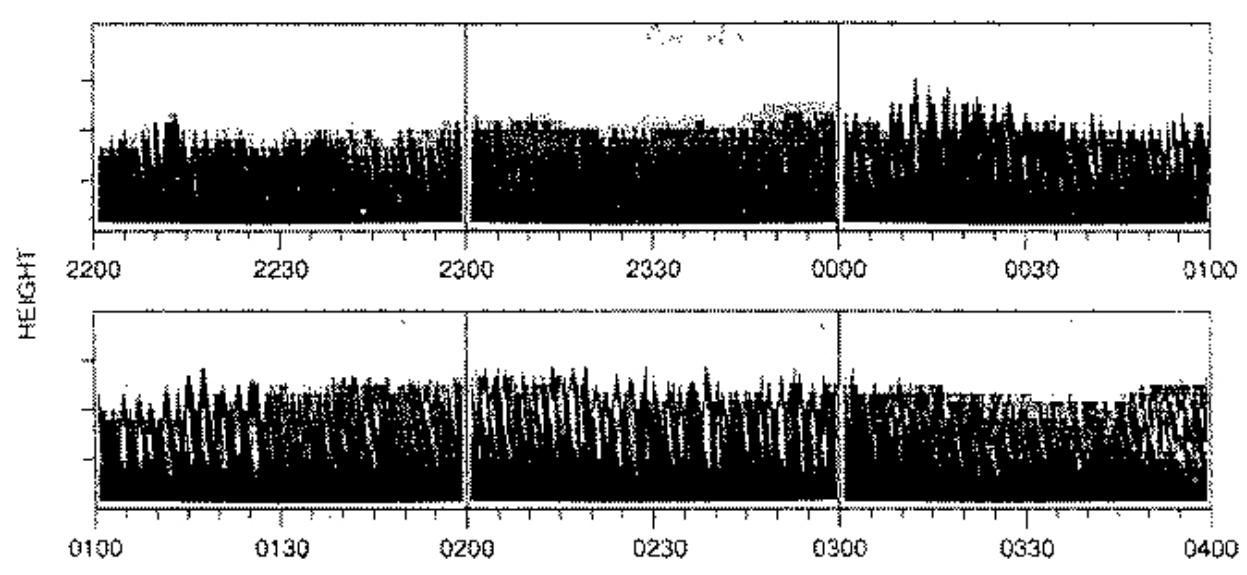

(a)

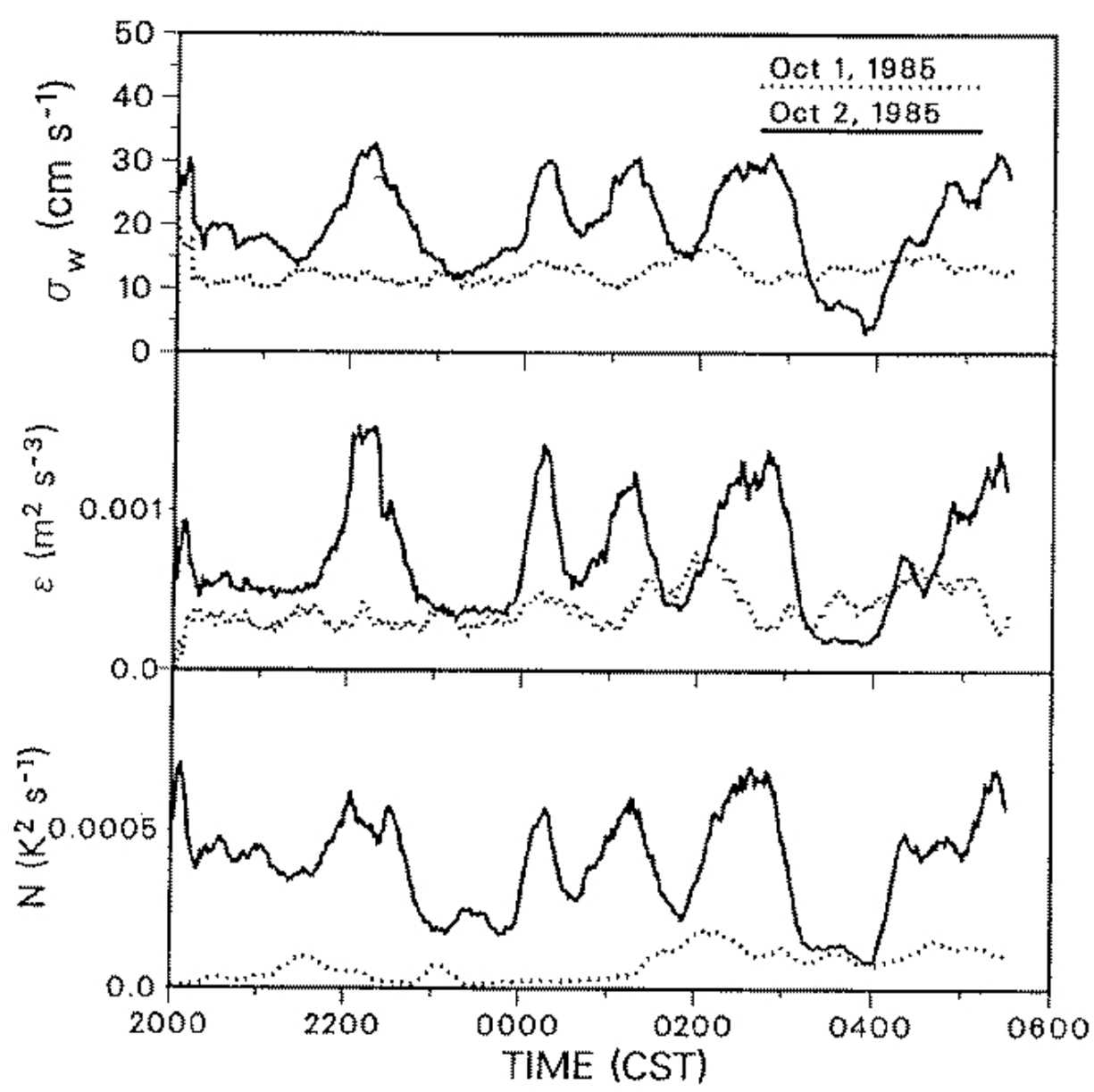

(b)

Figure 2.18: Observed SBL turbulent episodes: (a) time series of minisodar backscatter signal strength on night of October 2, where vertical axis tick marks are every $50 \mathrm{~m}$; (b) time series from October 1 and 2 of vertical velocity RMS (top panel), dissipation rate (middle panel), and temperature variance dissipation rate (lower panel); (after Coulter, 1990). 


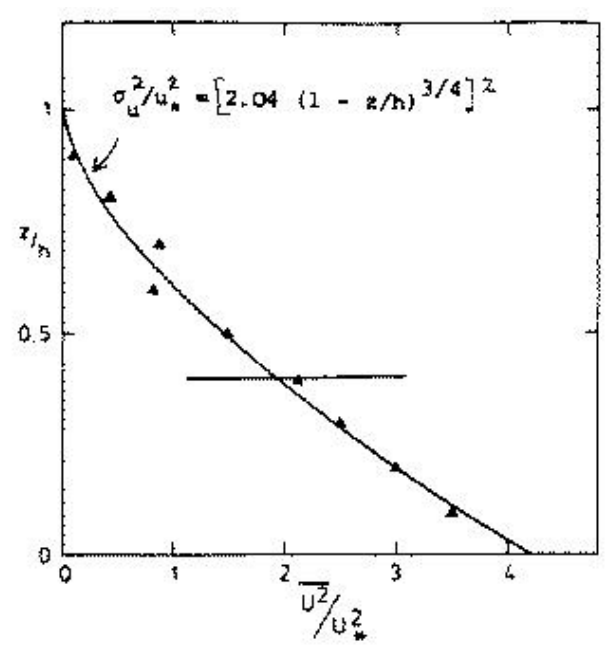

(a)

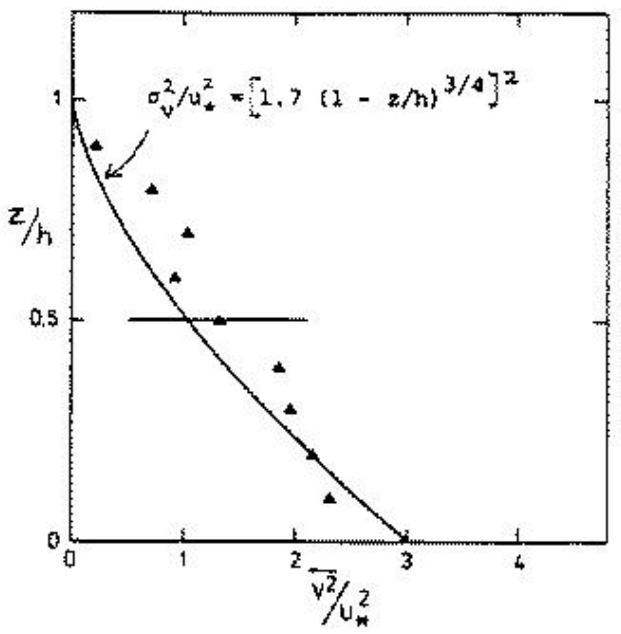

(b)

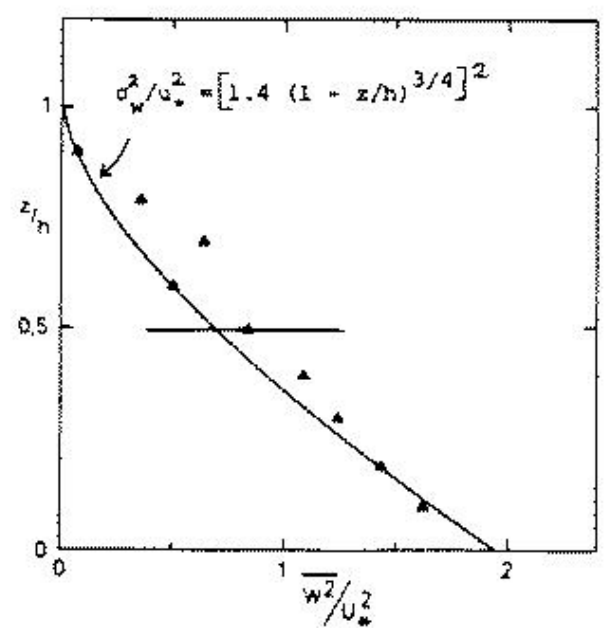

(c)

Figure 2.19: Nondimensional profiles of average velocity variances at the Cabauw tower; see Figure 2.17 for explanation of symbols (after Nieuwstadt, 1985). 


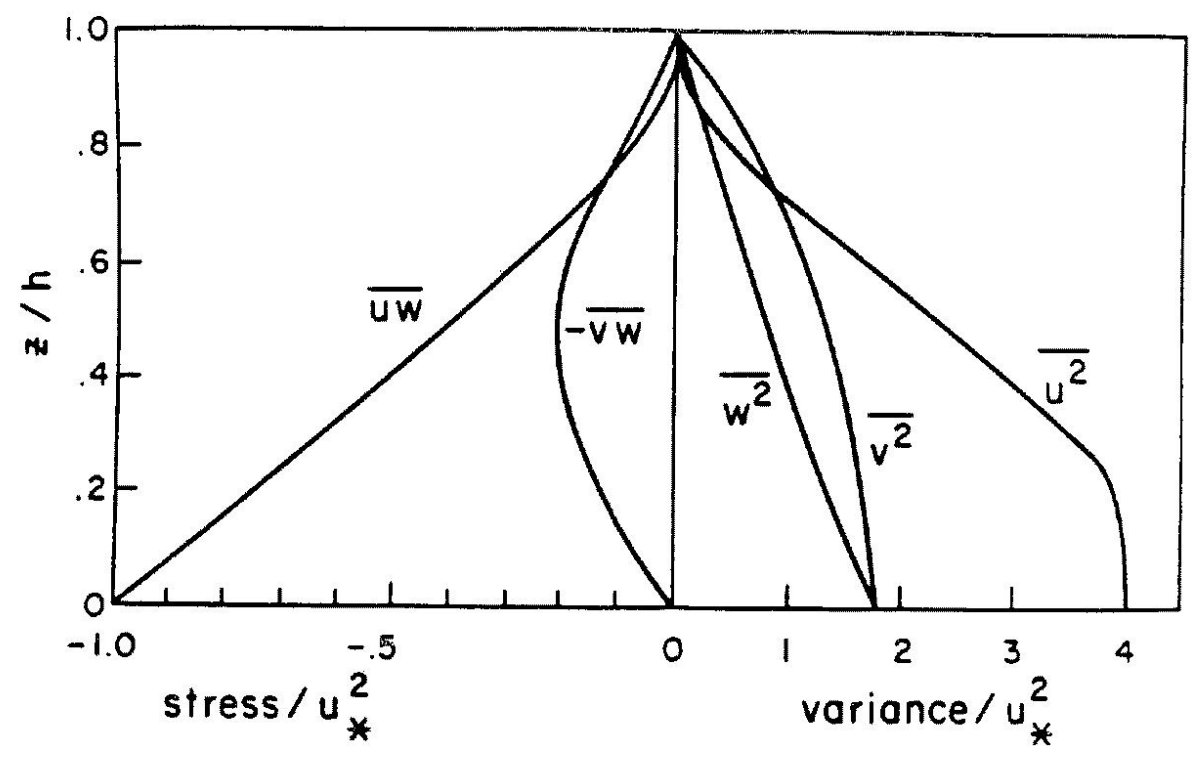

Figure 2.20: Nondimensional profiles of turbulence quantities for the SBL (after Wyngaard, 1975). 
(a)

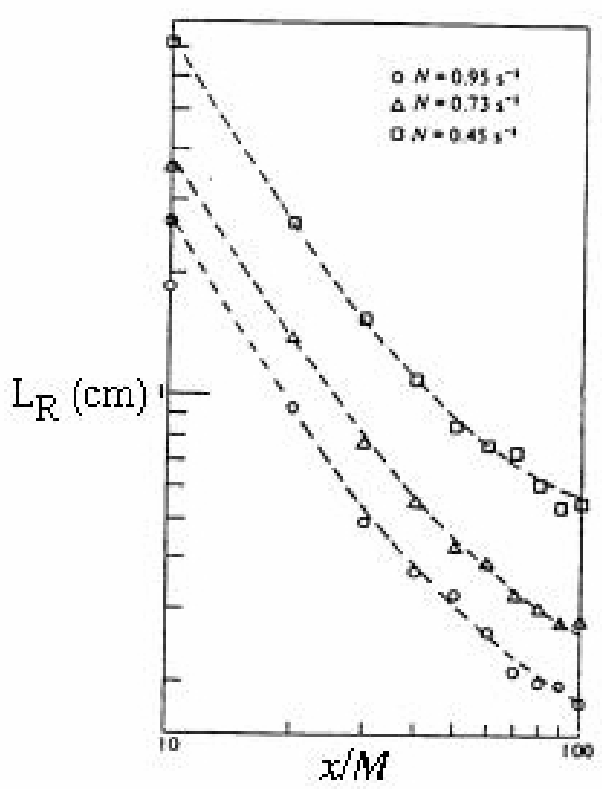

(b)

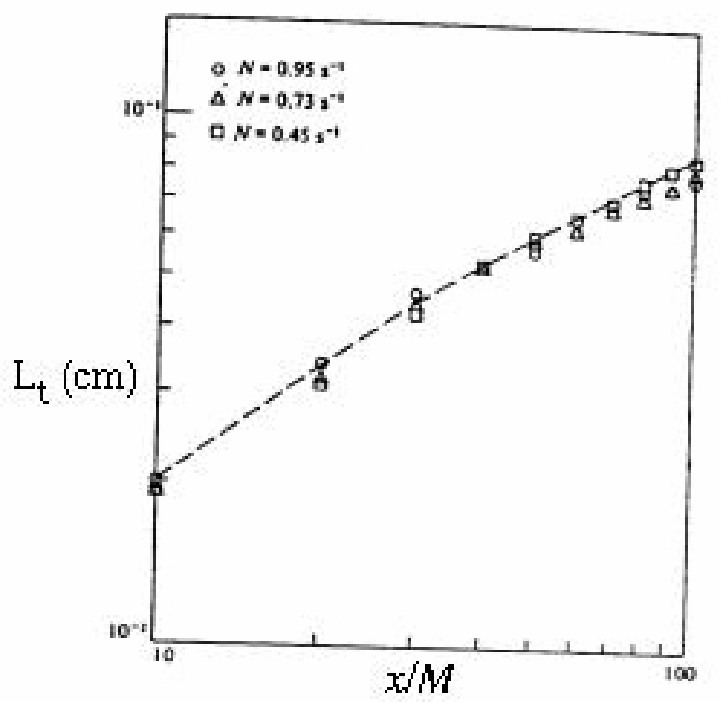

(c)

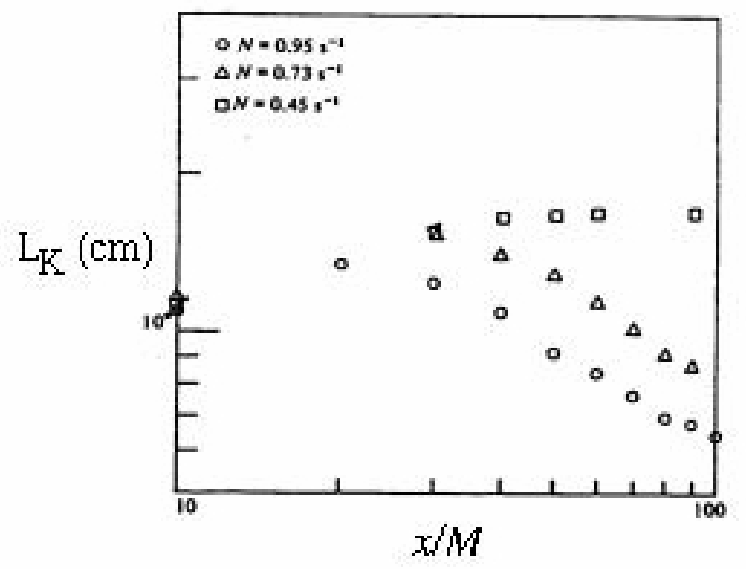

Figure 2.21: Downstream evolution under three stabilities in Run 23 for (a) Ozimidov length scale, (b) Kolmogorov length scale, and (c) turbulent length scale; downstream distance $\mathrm{x}$ is normalized by the grid mesh size M (after Stillinger, et al., 1983). 


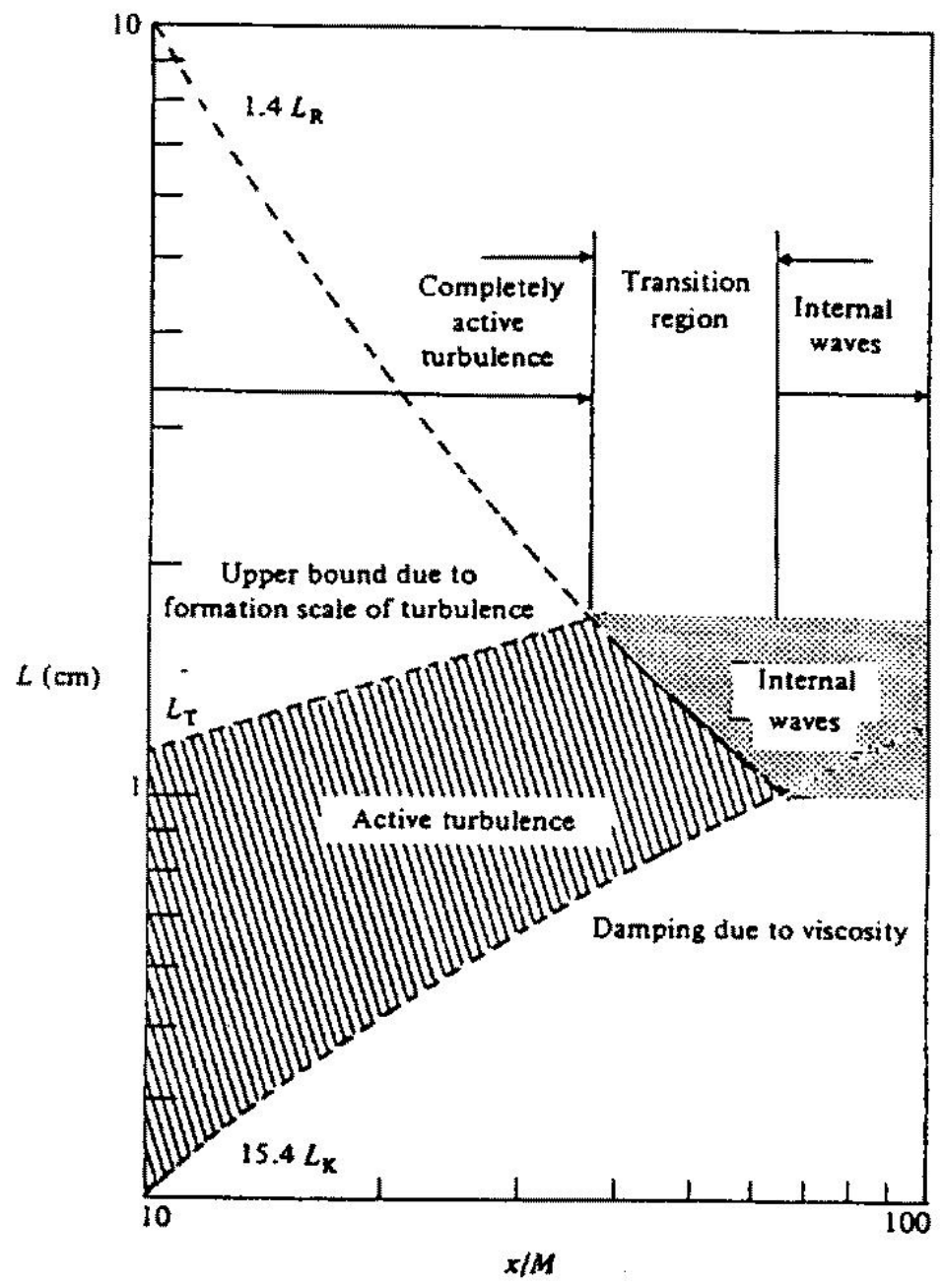

Figure 2.22: Evolution map of homogeneous turbulence in a stably stratified fluid; $M$ is mesh size (after Stillinger, et al., 1983). 


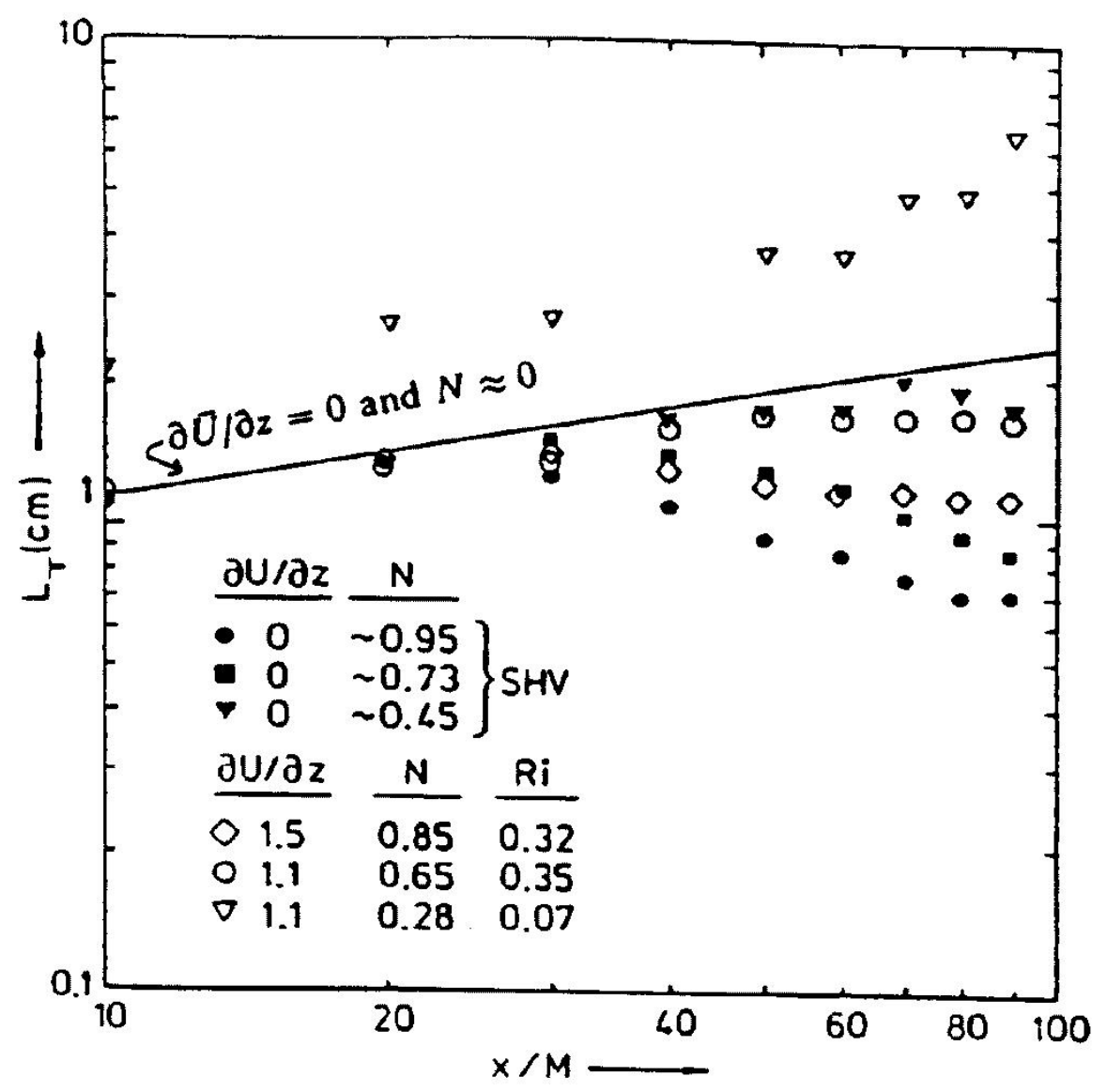

Figure 2.23: Overturning turbulent length scale vs. normalized downstream distance; solid symbols from Stillinger, et al., 1983; solid line from Montgomery (1974); open symbols from present experiment (after Rohr, et al., 1987). 


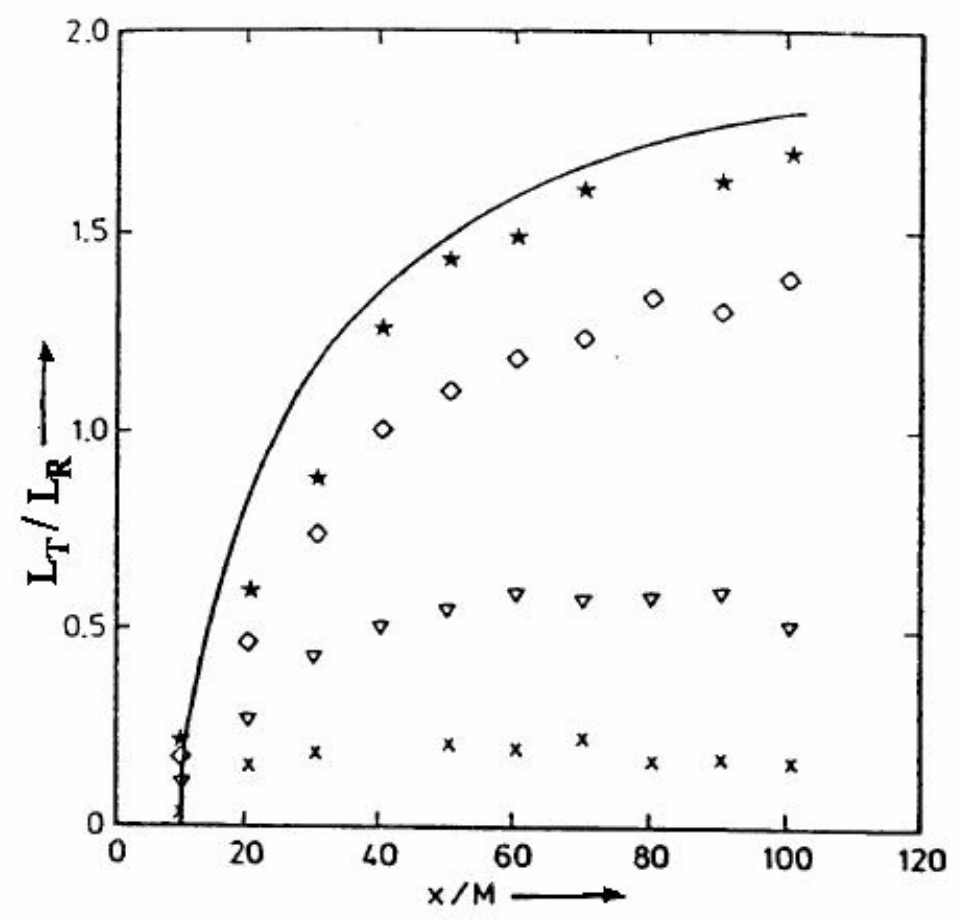

$$
\begin{gathered}
\text { Symbols } \\
-\begin{array}{c}
N=0.96 \\
\text { (unsheared). }
\end{array} \\
\star, N=0.65 \mathrm{rad} / \mathrm{sec} \\
\circ, N=0.49 \mathrm{rad} / \mathrm{sec} \\
\nabla, N=0.29 \mathrm{rad} / \mathrm{sec} \\
\times, N=0.16 \mathrm{rad} / \mathrm{sec}
\end{gathered}
$$

Figure 2.24: Downstream evolution of the ratio of observed to limiting overturning scales $\left(L_{T} / L_{R}\right)$; downstream distance normalized by grid mesh size M (after Rohr, et al., 1987). 


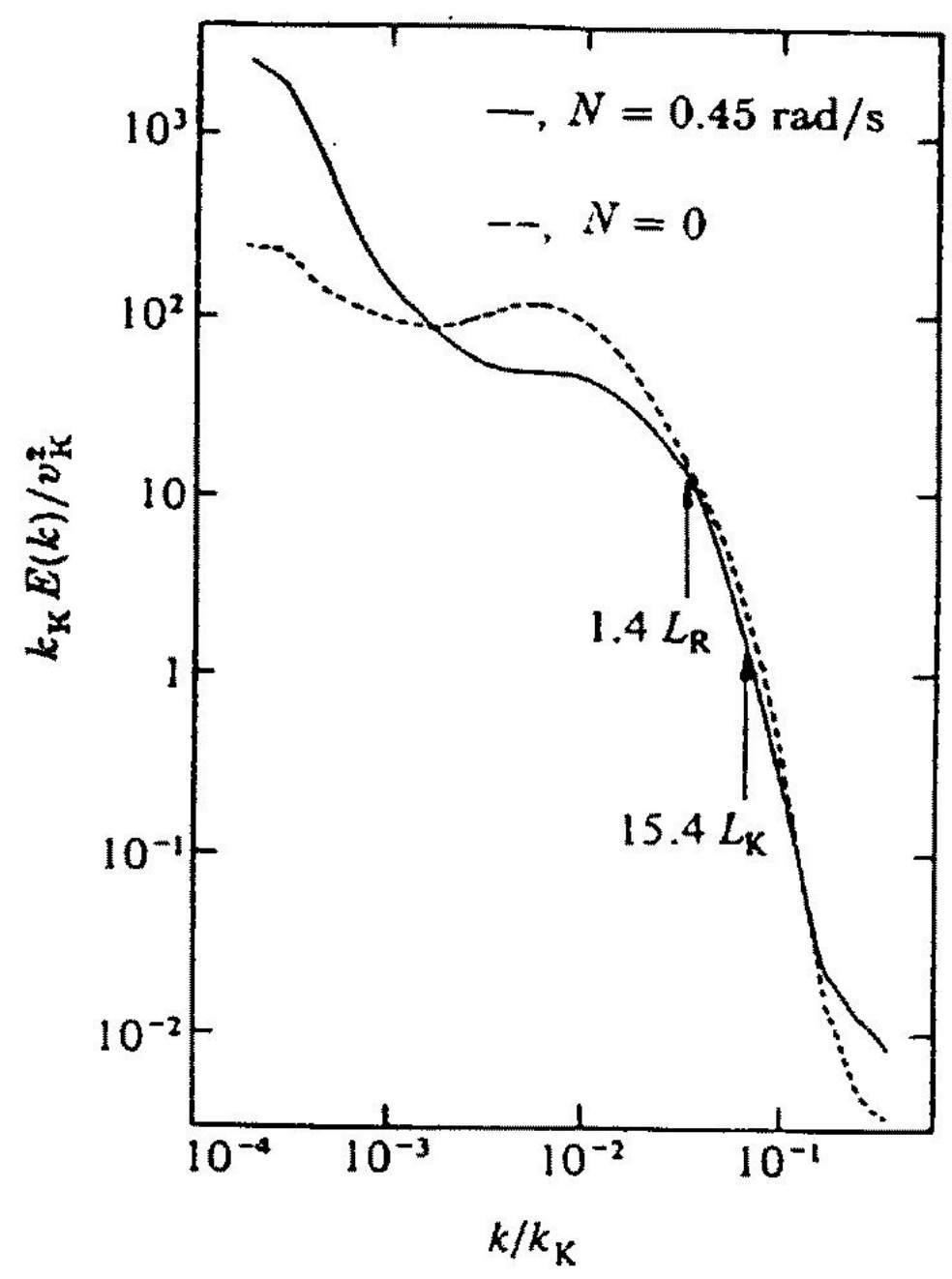

Figure 2.25: Normalized vertical velocity spectra at downstream distance $\mathrm{x} / \mathrm{M}=40$ for stratified and unstratified conditions (after Stillinger, et al., 1983). 


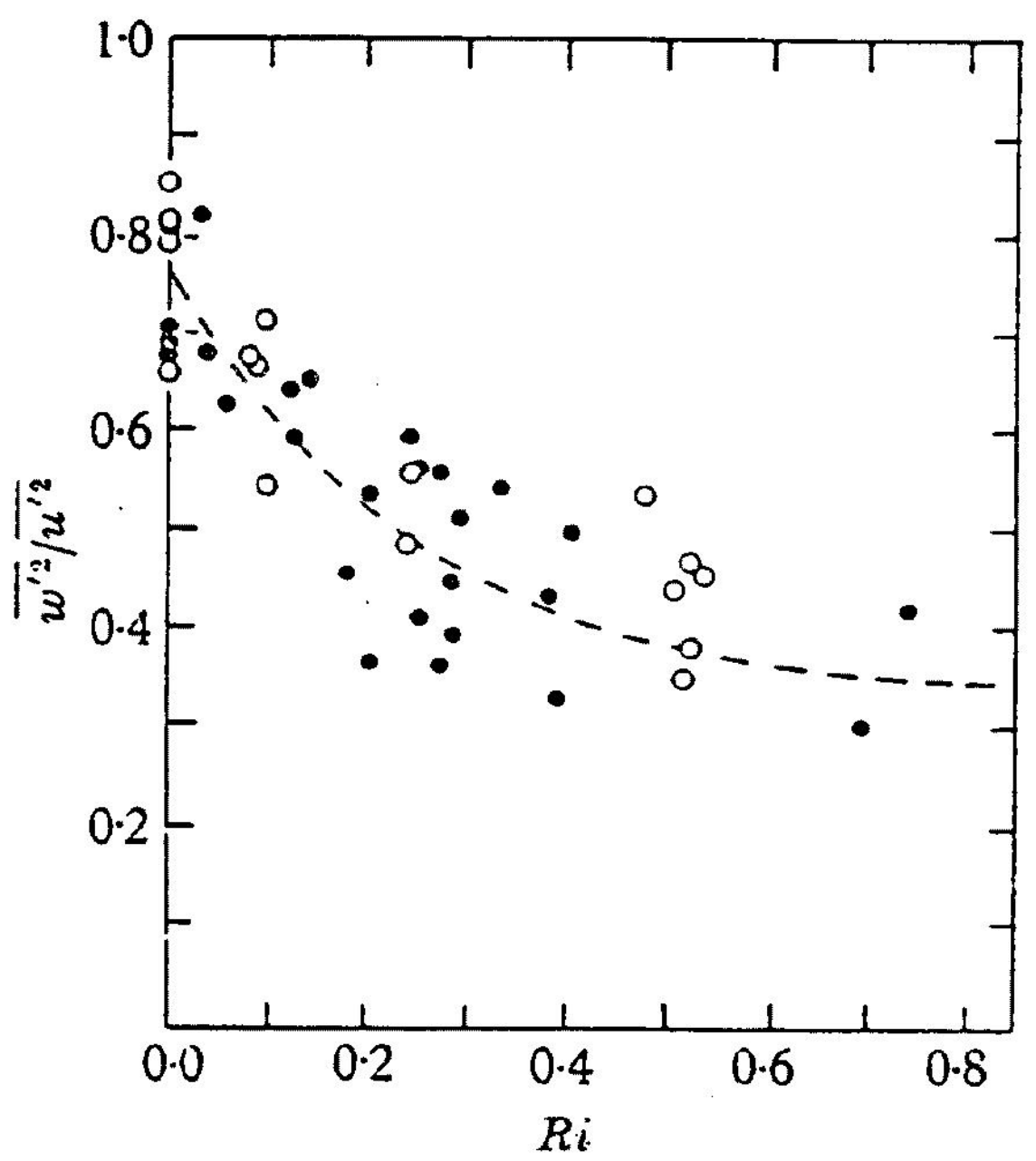

Figure 2.26: The ratio of $u-$ and w-velocity variances vs. Richardson number Ri; data from a wind tunnel measured along the centerline at a normalized downstream distance $\mathrm{x} / \mathrm{M}=66$ (after Webster, 1964.) 

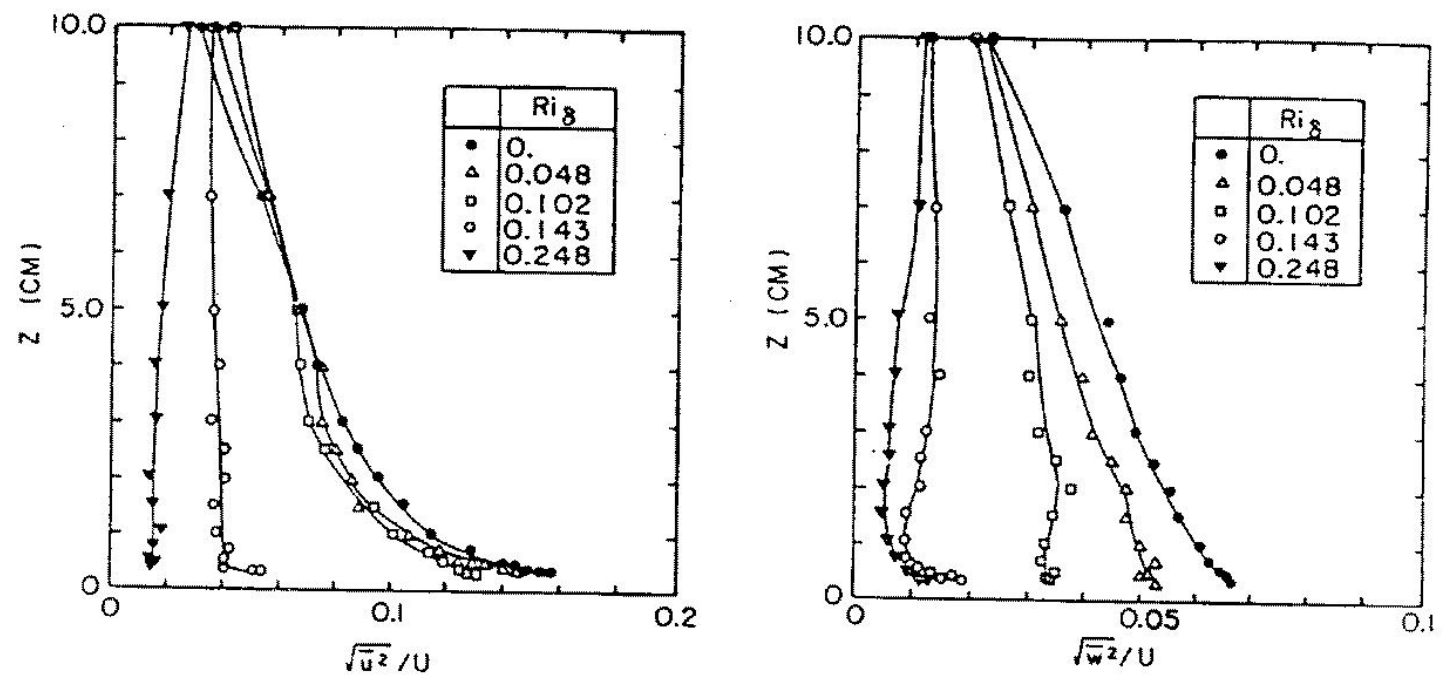

Figure 2.27: Profiles of turbulent intensity under five stability conditions for the (a) longitudinal and (b) vertical velocity components (after Ogawa, et al., 1985). 


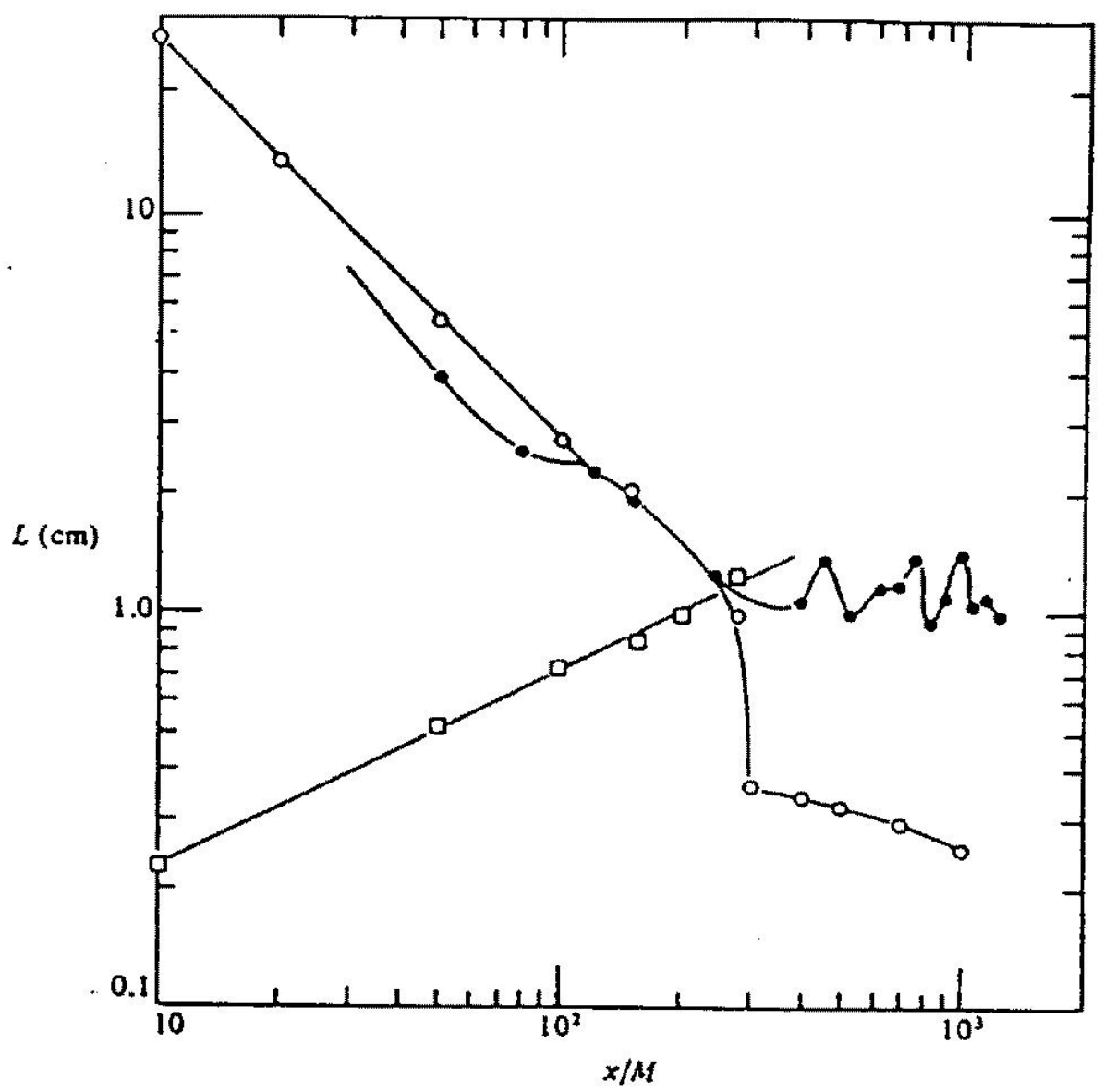

Figure 2.28: Length-scale evolution map for data of Dickey and Mellor (1980) for downstream distance normalized by grid mesh size M: $L_{b}$ (closed circles), $1.4 L_{K}$ (open circles), $15.4 L_{K}$ (squares) (after Stillinger, et al., 1983). 


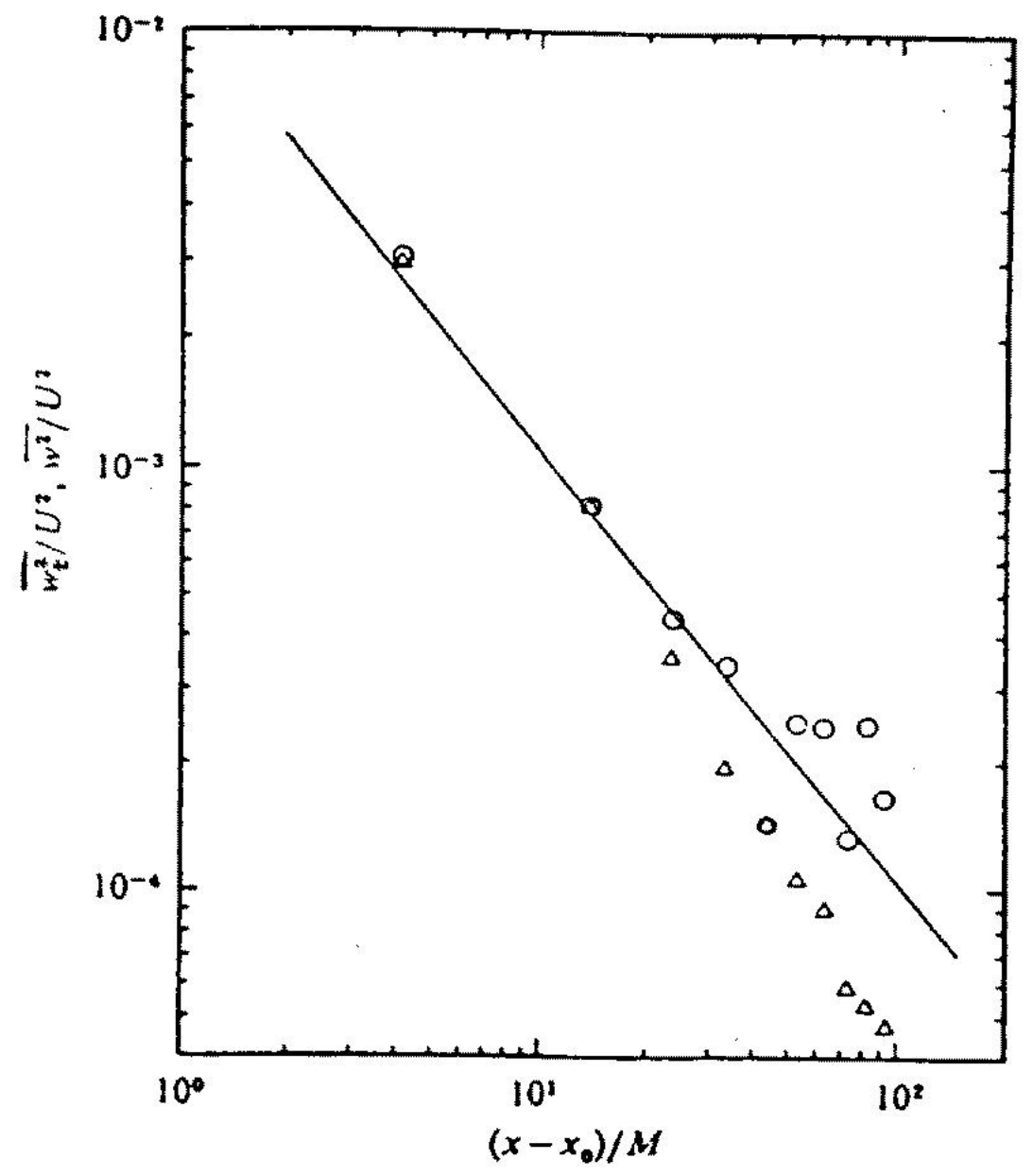

Figure 2.29: Comparison between the downstream evolution of total (circles) and turbulent (triangles) vertical velocity variance normalized by mean $\mathrm{KE}$ as estimated from data of Stillinger, et al., 1983; solid line is evolution of normalized vertical velocity variance without stratification (after Itsweire, et al., 1986). 

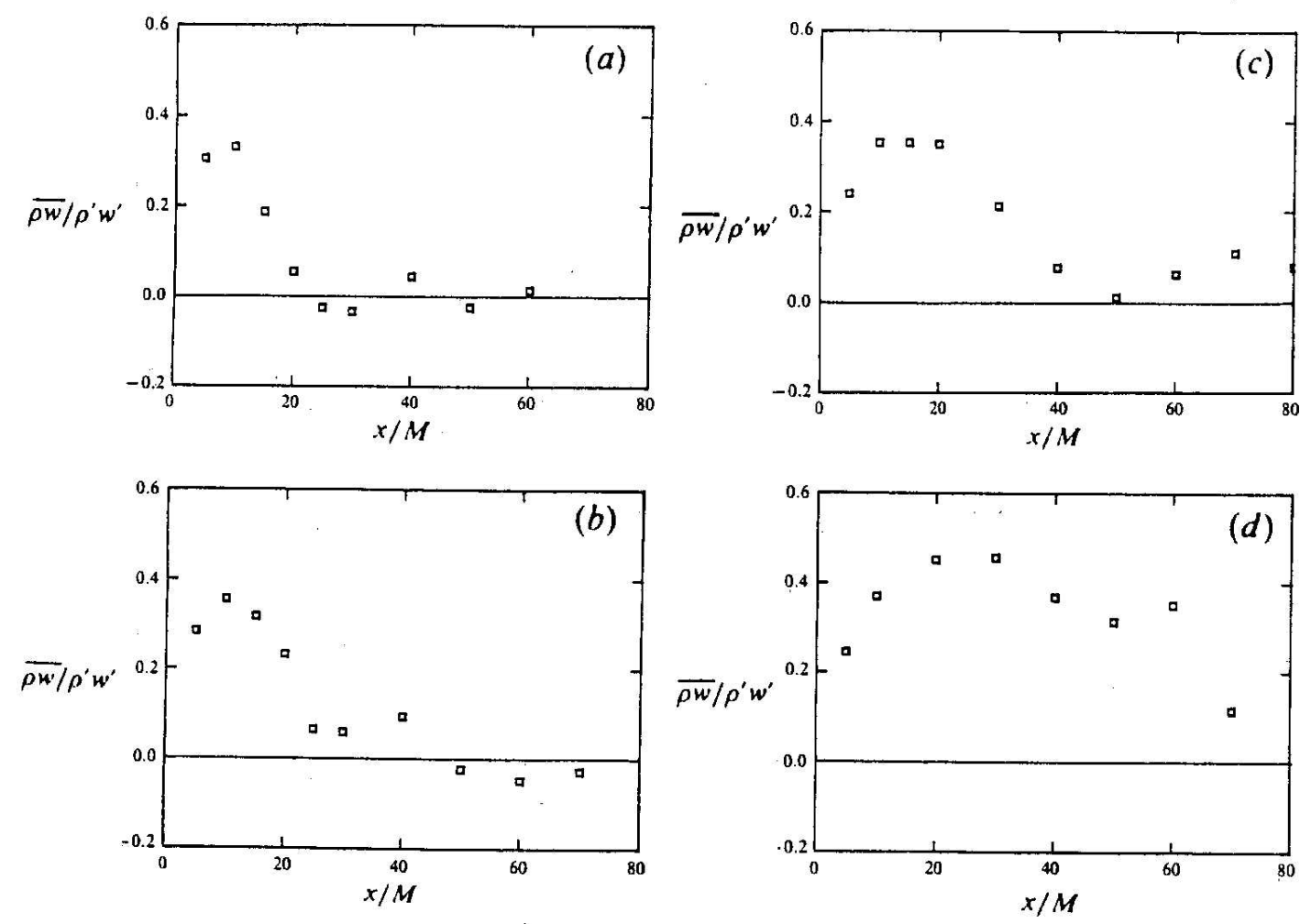

Figure 2.30: Normalized buoyancy flux vs. normalized downstream distance for four stabilities: (a) $\mathrm{N}=0.98 \mathrm{rad} / \mathrm{s}$, (b) $\mathrm{N}=0.74 \mathrm{rad} / \mathrm{s}$, (c) $\mathrm{N}=0.44 \mathrm{rad} / \mathrm{s}$, and (d) $\mathrm{N}=0.24$ $\mathrm{rad} / \mathrm{s}$ (after Itsweire, et al., 1986).
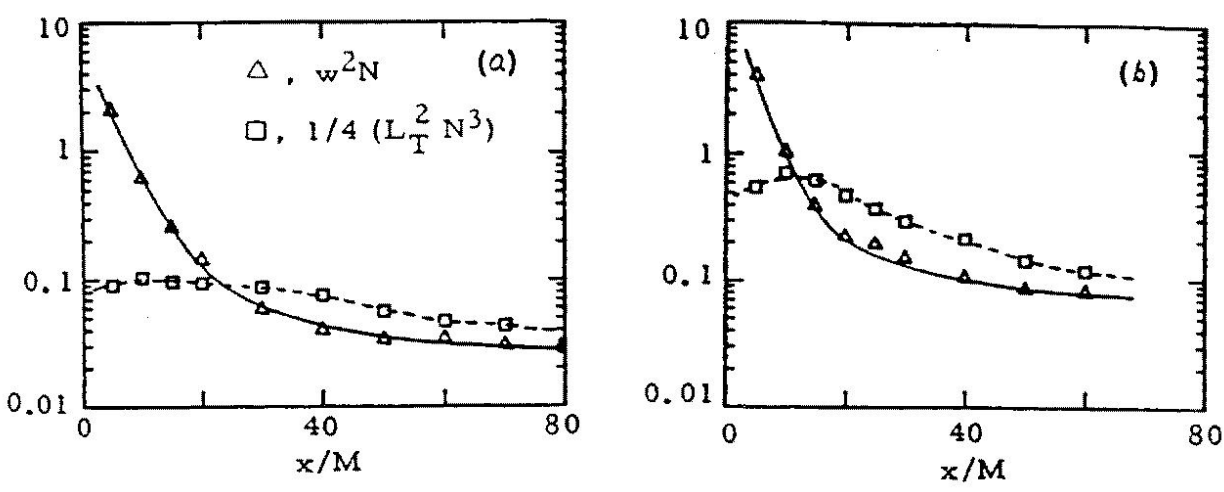

Figure 2.31: Evolution of vertical KE (triangles) and fluctuating PE (squares) for (a) N $=0.43 \mathrm{rad} / \mathrm{s}$ and (b) $\mathrm{N}=0.97 \mathrm{rad} / \mathrm{s}$ (after Van Atta, et al., 1984). 


\section{Chapter 3}

\section{Turbulence Modeling Approaches}

Turbulent flow is described by the Navier-Stokes equations, which are based on the conservation of momentum. A general solution is not available which can predict the time-dependent behavior of variables characterizing turbulent flows. However, several levels of approximation have been developed, especially with the recent growth of computer capability, which perform reasonably well in predicting the observed behavior of a number of well-defined turbulent flows. This section will address the current state of these approximations (models) used for predicting turbulent flow in the PBL.

The most widely used approach has been a statistical one, attributed to Reynolds, in which the Navier-Stokes equations are averaged over a time scale much longer than that associated with turbulence in the flow. These averaged equations predict mean values for variables, and characterize turbulent processes by averaged products of fluctuating values of the variables. The difficulty in this approach is that there are no equations in the original set to account for the averaged fluctuation products (turbulence terms) which arise from Reynolds-averaging. To close the system, one must add a sufficient number of algebraic or differential equations to account for the additional terms. These new equations (turbulence models) express the needed solutions in terms of variables in the averaged set of equations and, to varying degrees, use empirical relationships which relate the distribution of these variables to the turbulence terms.

Another approach is to average (filter) the Navier-Stokes equations over some small interval, such as one or more cells of a computational grid. The grid size is small enough so that large eddies, assumed to contain most of the turbulent energy, are explicitly calculated; the influence of small, subgrid-scale (SGS) eddies are then parameterized by simpler schemes. This approach, known as Large-Eddy Simulation (LES), is feasible because of the rapid increase in computer computation capability. 


\subsection{Background}

A large number of turbulence models have been proposed since Reynolds first suggested his statistical approach. A majority of these models can be divided into two closure categories as follows. Those models which approximate turbulence terms by reference to mean fields of variables are categorized as first-order closure models. The level of sophistication in this type of model is quite variable, ranging from a simple algebraic expression to two partial differential equations. Models in the other category, second-order closure, have transport or algebraic equations for the turbulence terms in terms of the turbulence terms themselves. The resulting equations introduce higherorder terms which must be approximated in terms of lower-order quantities to close the system. Second-order closure models can become quite complicated, involving up to 13 partial differential equations for the turbulence terms. Some simplification is achieved by substituting algebraic equations for some of the differential equations.

A major deficiency in Reynolds-averaged equations is that all scales of turbulence must be modeled in the closure approximation, since all of the turbulence is averaged simultaneously (Ferziger, 1977). Large-scale structure in turbulent shear flows was experimentally established in the mid-1970s; the behavior of these structures was much more deterministic than previously thought (Kovasznay, 1978). However, most turbulence models do not explicitly incorporate large-scale structure, using instead statistical approaches which lump all scales together; this is changing as many experimental and theoretical efforts are addressing large-scale structures in turbulent flows (Reynolds, 1976).

At high Reynolds number, turbulence can be thought of as a superposition of multiple-scale eddies (Schumann et al., 1980). Observations of simple to moderatelycomplex turbulent flows show that the large eddies are responsible for the majority of turbulent transport (Kovasznay, 1978). The large-eddy simulation modeling approach, to be discussed in this and subsequent chapters, calculates the large eddies explicitly and models the small eddies which cannot be resolved in the calculation scheme.

Laboratory studies of mixing layers and boundary layers, discussed in Subsection 2.2.1, suggest that these flows are comprised of organized large-scale eddies 
containing most of the energy absorbed from the mean flow. Ferziger (1981) has contrasted the properties of these eddies with the small eddies which occur simultaneously. The large eddies interact strongly with the mean flow and are responsible for most of the turbulent transport; the small eddies interact nonlinearly with the large eddies and are responsible for dissipation of the turbulent energy they receive. The shape and strength of large eddies are dependent on the nature of the flow, with frequent occurrence of high anisotropy; small eddies are much less flow dependent, much more nearly isotropic, and hence more nearly universal. The time scales of large eddies are similar to those of the mean flow; small eddies have much shorter time scales.

Given these characteristics, it seems promising that one could successfully model the small-scale eddies which are more universal and carry less energy than large-scale eddies. It is clear, from the properties given above, that the large eddies would be very difficult to model successfully in anything but the simplest flows. LES optimizes this situation by calculating the large eddies directly and modeling the small eddies.

Unfortunately, there is no distinction between the large and small eddies that would work a priori for all flows. The zones for production and dissipation of turbulent energy tend to occur separately at low and high frequency respectively; in addition, if one assumes that small eddies are isotropic, there is very weak coupling between large and small eddies (Cantwell, 1981). At high Reynolds number there is a range of eddy sizes called the inertial subrange, where production and dissipation do not directly affect eddy motion and energy is transferred inertially from larger to smaller scales (Tennekes and Lumley, 1972). This would be a good, physically-based point to delineate between energy-producing large eddies and energy-dissipating small eddies. The difficulty is that often the domain of interest is large, and explicit calculation down to such small scale in the inertial subrange may be impractical. In this case, the division between scales of large and small eddies must be arbitrarily larger than the inertial subrange; this results in some turbulent energy production in the "small" (unresolved) eddies. Use of the inertial subrange as a dividing point, however, has been shown to be an unnecessarily small scale; thus choosing a point at slightly larger scale should still give satisfactory results (Ferziger, 1977). 


\subsection{SGS Turbulence Models}

When using the LES approach, there are two commonly used methods of separating large and small eddies at some specified point. The first method is to filter explicitly the governing equations; low-frequency (large) eddies are preserved for explicit calculation, while high-frequency (small) eddies are "removed" and their contribution modeled. The choice of filter type is of minor importance; box and Gaussian filters are commonly used. However, Piomelli et al. (1987) have found that the choice of filter type must be consistent with the type of SGS turbulence model, so that both have the same length-scale information. Filter width is of more importance, since the filter must be large enough to insure accuracy, yet small enough to resolve features of interest in the flow; the optimum filter width is about 2-4 grid cells (McMillan and Ferziger, 1979). The second method is to filter the governing equations implicitly when they are discretized according to a specified grid, and average quantities over grid control volumes. A problem with this method is that if a variable has large gradients, discontinuities will occur at the boundaries of control volumes. The first method is preferable in this regard, since it smoothes the behavior of Reynolds stresses across the grid; it is also independent of grid specification at the outset (Ferziger, 1981).

The use of filtered equations introduces an additional complexity. The rules of Reynolds-averaging lead to ${\overline{u_{i}}}_{j}=\bar{u}_{i} \bar{u}_{j}+\overline{u_{i}^{\prime} u_{j}^{\prime}}$ when the averaging is uniform over an unbounded, homogeneous domain or is an ensemble average. In the case of averages of bounded domains, as done for filtered equations, the Reynolds-averaging rules do not apply (Leonard, 1974). The terms $\overline{\bar{u}_{i} u_{j}^{\prime}}$ and $\overline{u_{i}^{\prime} \bar{u}_{j}}$ are not zero; if these terms are zero, then $\overline{\bar{u}_{i} \bar{u}_{j}}=\bar{u}_{i} \bar{u}_{j}$. Hence, the effects of $\overline{\bar{u}_{i} u_{j}^{\prime}}$ and $\overline{u_{i}^{\prime} \bar{u}_{j}}$ can be represented as the residual $L_{i j}=\overline{\bar{u}}_{i} \bar{u}_{j}-\bar{u}_{i} \bar{u}_{j}$, called the "Leonard stresses".

The choice of turbulence model for simulating SGS eddy motion is not nearly as critical in LES as in Reynolds-averaged equation solution. This is primarily true because SGS modeling does not have to account for all scales of turbulence, but rather just for very small scales. Anisotropy, turbulent transport, pressure fluctuations, and pressure-strain correlations are associated mainly with large eddies. This allows the successful use of simple eddy viscosity models for small eddies. Comparison studies have shown that, for homogeneous turbulence, more sophisticated two-equation 
and full Reynolds stress models do not provide significant improvement over simple models (Clark et al., 1979; Knight and Murray, 1980). Inaccuracies arise more from misalignment of the principal axes of the SGS stress tensor and the large-scale strain-rate tensor than from SGS models (Clark et al., 1979). SGS models perform satisfactorily in homogeneous turbulence and in flows with weak strain, especially if an anisotropic filter is used (McMillan and Ferziger, 1979). Results are much less satisfactory for inhomogeneous turbulence, particularly near boundaries where small scales become anisotropic (Ferziger, 1981).

A wide range of closure models, from full second-order models to simple eddy viscosity models, have been used to treat SGS turbulence in LES. From Deardorff's landmark runs in the early 1970's, it became clear that the computational burden of full second-order equations for the SGS was not justified. On the other hand, Schemm and Lipps (1976) argue that simple eddy viscosity models that assume local equilibrium are inadequate for treating strongly stratified conditions where countergradient heat flux may occur.

The advantages of LES over statistical turbulence models (first- and secondorder closure) have been summarized as follows by Schumann et al. (1980). Threedimensional effects are accounted for explicitly. Results for turbulence are predictive since important time-dependent phenomena are being calculated implicitly. Pressure is more accessible in this approach. The simple SGS models are more intelligible and do not suffer problems of realizability (i.e. negative energy predictions). Phenomena of bursting and intermittency are resolvable.

The features of the types of several SGS models currently in use are summarized in the next subsections. Recent reviews can be found in Lesieur and Metais (1996), Piomelli (1999), and Sagaut (2001).

\subsubsection{Eddy viscosity models}

The earliest SGS model applied to the atmosphere was that developed by Smagorinsky (1963). Here the SGS model followed the idea of the Reynolds-avergaged first order closure, where the eddy viscosity is proportional to gradients of the mean velocity field, $\nu_{t}=\left(C_{s} \Delta\right)^{2}\left(\overline{S_{i j}}\left(\overline{S_{i j}}\right)\right.$, where $C_{s}$ is the Smagorinsky coefficient. This coefficient 
has a theoretical value of about 0.2 (Lilly, 1967), but is often set to smaller values, especially near the surface, to give better results. Use of the grid scale $\Delta$ as the SGS length scale reflects the notion that the grid resolution determines the size of the eddy motions resolved in the LES. A basic assumption of the Smagorinsky SGS model is that the SGS stress is aligned with the resolved strain rate tensor. A priori tests with the Smagorinsky SGS model show that it has very low correlation with SGS stresses obtained from direct numerical simulation (DNS) where the flow is fully resolved and no SGS model is used. In spite of this, use of the Smagorinsky SGS model in atmospheric simulations has led to surprisingly good results, especially for the convective boundary layer where the energy-containing convective eddies are well resolved.

Deardorff (1980) extended the Smagorinsky model by introducing a SGS velocity scale based on a prognostic equation for the SGS TKE. Effects of thermal stratification and the transport of SGS TKE are included in this approach. It is basically a MellorYamada 2.5 level model applied at the subgrid scale. This model has been used in several LES models of the atmospheric boundary layer.

The fluctuating velocity field must be resolved adequately when using LES. Approaching the ground, this can become difficult when important eddy motions occur at smaller scales. Hence the stress predicted by the dynamic SGS model may be inconsistent with the value prescribed at the bottom boundary condition by the similarity theory. Such a problem would lead to unrealistic values of the vertical stress divergence and hence unrealistic velocity accelerations. The problem of matching stresses from the outer region with those in the region near the ground has been addressed by Sullivan, et al. (1994). They developed a two-part eddy viscosity model where the computation of the SGS stress is divided into an isotropic contribution that varies with magnitude of the fluctuating strain rate, and an inhomogeneous contribution that varies with magnitude of the mean strain rate.

Eddy viscosity models are entirely dissipative, that is, energy is transferred only from large scales to small scales. Analysis of DNS results for channel flows indicates that a fair fraction (but less than half) of the grid points experience upscale energy transfer (energy backscatter), and that the net energy transfer is downscale. In the next subsections, SGS models will be described that support both forward scatter 
and backscatter.

\subsubsection{Stochastic backscatter models}

Developers of stochastic backscatter argue that the treatment of backscatter as a stochastic process is more consistent with the relation between resolved and SGS eddies. Spectral theories suggest that the phase of energy backscatter should be random with respect to the mean shear (Mason, 1994). Deterministic SGS models to be discussed in the next subsections can have a tendency to systematically enhance gradients resolved in the LES; theory suggests that the energy should be transferred without such a systematic enhancement of resolved gradients.

Chasnov (1991) provides an extensive analysis of the theory and application of eddy viscosity and stochastic energy backscatter in homogeneous turbulence. He uses the eddy-damped quasi-normal Markovian (EDQNM) model to determine the damping and stochastic forcing terms for LES of the Kolmogorov inertial subrange. If backscatter is accounted for in LES by diminishing the damping term, as done in the Germano-type dynamic SGS model, the simulation does not produce the well-known Kolmogorov spectrum. On the other hand, the explicit use of stochastic backscatter gives a more realistic simulation of the Kolmogorov inertial subrange.

The initial stochastic backscatter SGS model was developed by Leith (1990). The backscatter (or upscale transfer) of SGS turbulent energy is represented as a stochastic process, since the SGS eddies induce a random forcing on the resolved scales of motion through nonlinear interactions.

Mason has used the Leith SGS backscatter model for LES of the PBL, with some modification to treat small eddies near the ground (Mason and Thomson, 1992). The results with backscatter show more realistic velocity profiles, especially near the ground. A typical problem in the past for LES of the shear-driven PBL has been the unrealistically large vertical gradients of velocity near the ground. With the use of stochastic backscatter in their SGS model, Mason and Thomson (1992) were able to resolve this difficulty without creating problems elsewhere in the PBL. Another benefit of using backscatter was the initial growth rate of TKE in the PBL simulation was more realistic. Without backscatter, there was an initial transient that would 
dramatically overshoot the expected level of TKE after a long development period of unrealistically low values. This is especially true for the shear-driven PBL. With backscatter, the initial growth rate shows a rapid rise to the expected level. Mason (1994) attributes the unrealistic growth (without backscatter) to the inability of the random fluctuations in the initial velocity field to extract energy from the mean flow. The presence of backscattered energy may help support the initial structure of random fluctuations so that energy is transferred more quickly from the mean flow into resolved eddies.

Schumann (1994) has developed a stochastic SGS model that differs from the Leith model. This new model has been formulated as an extension of the Smagorinsky (1963) SGS model, although it can be used with other SGS models. The model reduces the correlation between stresses and strains by introducing random stresses to their deterministic counterparts. The model provides realizable random Reynolds stresses and scalar fluxes since it uses a quadratic expression to give a positive definite stress tensor and to insure that the correlation coefficient between velocity and scalar fluctuations does not exceed unity. The model accounts for incompressibility effects of both the SGS motions and the random forcing on the resolved motions.

\subsubsection{Scale-similarity backscatter models}

Scale-similarity SGS models are based on the assumption that the smallest resolved scales and largest unresolved scales (SGS) are similar. Thus, information available in the smallest resolved scales can be used to estimate the dynamics at unresolved scales. The first scale-similarity was developed by Bardina, et al. (1983). This new form of SGS model had high correlations with exact SGS stress in a priori tests. The model demonstrated correct near-wall behavior (Sarghini, et al., 1999) and it is also Galilean invariant (Speziale, 1985). This model allows energy backscatter, but unfortunately does not have enough dissipation. To address this problem, Bardina, et al. (1983) added an eddy viscosity SGS model to their scale-similarity model to form a 'mixed' model; the eddy viscosity SGS model used was that of Smagorinsky. The scale-similarity part of the model provided correct representation of the turbulence where the Smagorinsky SGS model had been deficient; the eddy viscosity part 
was left to provide just enough dissipation without otherwise negatively impacting the simulation. The ability to incorporate backscatter via the scale-similarity approach provides an alternative to the stochastic approach described above, and thus to achieve the associated benefits without having to accept the assumptions required of the stochastic approach.

\subsubsection{Dynamic backscatter models}

Thus far, all the SGS models have coefficients that must be set. Although this allows for tuning to get best fits to measurements, it remains a liability as well. The scalesimilarity model of Bardina, et al. (1983) has been extended by Germano, et al. (1991) to develop a new SGS turbulence model. The new model is based on the algebraic identity between turbulent stresses at resolved scales and SGS stresses from velocity fields that are filtered at two different scales

$$
\Gamma_{i j}=T_{i j}-\tilde{\tau}_{i j}
$$

where $\Gamma_{i j}$ is resolved turbulent stress, and $T_{i j}$ and $\tilde{\tau}_{i j}$ are the SGS turbulent stress at the test and grid filters, respectively. The tilde $(\sim)$ represents the test filter, with

a characteristic width of $\widetilde{\bar{\Delta}}$, while the overbar $(-)$ represents the grid filter with a characteristic width of $\bar{\Delta}$. In practice, the test filter is applied to the grid-filtered fields. This gives the following definitions of the stress terms:

$$
\begin{gathered}
\tau_{i j}=\overline{u_{i} u_{j}}-\bar{u}_{i} \bar{u}_{j}, \\
T_{i j}=\widetilde{u_{i} u_{j}}-\widetilde{\bar{u}_{i}} \widetilde{\bar{u}_{j}}, \\
\Gamma_{i j}=\widetilde{\bar{u}_{i} \bar{u}_{j}}-\widetilde{\bar{u}_{i}} \widetilde{\bar{u}_{j}} .
\end{gathered}
$$

The Smagorinsky SGS model is used at each of the filtered scales to represent the SGS stresses. When the resulting expressions for SGS stress are combined, this yields a new coefficient $C_{g}$ that is similar to $C_{s}$. But now, the new coefficient is no longer 
constant, but rather is dynamically determined by the flow and is a function of time and space. Initially, in the Germano formulation, the coefficient $C_{g}$ was a function of height, but not the two horizontal dimensions, because averaging was required over the horizontal plane. The averaging is required to avoid indeterminate or ill-conditioned values of $C_{g}$. Since the coefficient is adjusted based on the dynamics of the resolved flow, this approach is commonly referred to as a dynamic SGS eddy viscosity model. This new model showed significant improvements over the basic Smagorinsky model, especially in predicting correct asymptotic behavior near the bottom surface.

The ability to select the best value for $C_{g}$ via a dynamic method is a welcomed improvement. In the past, several researchers have reported the importance (and associated difficulty) in choosing the correct (fixed) value of $C_{s}$ prior to the run. Yoshizawa (1989) developed a variable $C_{s}$ model to deal with this, but the model did not account for backscatter. In a test of four different LES models (Nieuwstadt, Mason, Moeng, and Schumann, 1991), the largest source of differences in the model results was attributed to the values used for $C_{s}$.

The requirement to average $C_{g}$ across a plane limits the applicability of the dynamic SGS model to strongly homogeneous flows. Such a model is not sensitive to any inhomogeneous behavior in the plane of averaging. Lund (1991) attempted to introduce local averaging. As part of his work, he analyzed the results of DNS runs averaged to evaluate what the values of the dynamic coefficient $C_{g}$ should be. The analyses show that indeed the values of $C_{g}$ at individual points are extremely noisy (varying by orders of magnitude). The coefficient of variation (rms/mean) is about 10. When large negative values of $C_{g}$ are used, the LES models developed unrealistically large fluctuations in the flow field; the run usually blew up shortly thereafter. Clearly some averaging is required, while still retaining some sensitivity to local features. Lund went further to investigate an erroneous assumption in the Smagorinsky-type model, where an isotropic relation is assumed between the SGS stress and the strain rate. In his analysis, Lund found that on a point by point basis, the isotropic alignment of stress and strain is not valid. He used the principal components of the stresses and strains to perform the alignment. This allowed the appropriate amount of anisotropy to be introduced, and markedly improved the assigned values of $C_{g}$. 
Other work has been done to incorporate local averaging. Lilly (1992) eliminated the need for plane averaging of $C_{g}$ by introducing a least squares technique to optimize the stress-strain relationship. Wong (1992) extended the work of Germano and Lilly by relating the SGS stress to the SGS TKE instead of the Smagorinsky relationship to the strain rate. Wong presents three models: (1) a linear isotropic model using a SGS TKE equation, (2) a nonlinear, anisotropic model using a SGS TKE equation, and (3) a nonlinear, anisotropic model that uses algebraic relationships to eliminate the need for the SGS TKE equation. The relationship of the SGS stress and the SGS TKE is especially important where the buoyancy is strong.

Finally, work has been done at Stanford to develop a dynamic SGS model that successfully uses local averaging (see Zang,et al., 1993). The SGS model is based on the mixed model of Bardina, et al. (1983), instead of the Smagorinsky model; hence, the model does not require alignment of the SGS stress tensor and the strain rate tensor. The new model is called a dynamic mixed model, rather than a dynamic SGS model. An attractive feature of the mixed model is that a major part of the energy backscatter is provided by the scale-similarity term involving the resolved scale. This means that the dynamic coefficient is not responsible for so much of the backscatter and hence will be less noisy. The Leonard term is modified and is calculated explicitly, so that only the residual terms must be modeled with the dynamically adjusted $C_{g}$. The calculated values of $C_{g}$ are averaged locally over a the test-filtering volume that includes all grid cells adjacent to the cell under consideration. This local averaging procedure is almost always successful. However, in the limited number of instances where the averaged $C_{g}$ values are negative beyond a magnitude that the model can handle, a cutoff is used. The cutoff is defined such that the total of the eddy and molecular viscosities is always non-negative. The new model has been used in LES of lid-driven cavity flow in laminar, transitional, and turbulent conditions; the results compare well with results of previous experimental and modeling studies.

Next, Salvetti added a dynamic coefficient for the modified Leonard term in Zang's model (see Salvetti and Banerjee, 1995). Since there are now two dynamic coefficients, the new model was called a dynamic two-parameter model (DTM). 


\subsubsection{Velocity Estimation Methods}

A final approach has been developed that does not require any models. Here the SGS velocities are estimated from the resolved velocities. The result is a scale-similarity type SGS model, and, in fact, the Bardina model is the lowest order of this form of model. An early version of this approach was introduced by Shah and Ferziger (1995), called the stimulated small scale model, that used an inverse filtering or deconvolution to obtain an estimate of the SGS velocity.

Domaradski and colleagues (Domaradski and Saiki, 1997; Domaradski and Loh, 1999; Loh and Domaradski, 1999) have also developed a method for estimating the SGS velocities from resolved-scale quantities. They have generalized the scalesimilarity approach of Bardina for use with spectral methods. Geurts (1997) has developed a generalized scale-similarity model with a polynomial used to approximate the inverse filtering. More recently, Stolz and Adams (1999) have presented their approximate deconvolution method which is similar to that of Geurts (1997). Katapodes, et al. (2000) use successive inversion of a Taylor-series expansion of the resolved velocity to estimate the SGS velocity. The velocity estimation methods that lead to scale-similarity models require that the interaction between resolved and SGS velocities occur near the filter cutoff scale. For low Reynolds number flows investigated experimentally, this is the case (Domaradski and Saiki, 1997). It is not clear whether this requirement would be met in the high Reynolds number flows characteristic of the SBL. 


\section{Chapter 4}

\section{Simulations of the SBL}

Certain features of the nocturnal, stable boundary layer (SBL) complicate the modeling of its structure and evolution, especially compared with the daytime, convective boundary layer (CBL). The SBL is generally non-steady throughout the night, although it appears to undergo rapid development after sunset, followed by more gradual development. In strongly stable conditions, turbulence may become intermittent or episodic. Turbulent mixing, especially in the vertical, is reduced by the stable stratification to the point sometimes where vertically adjacent horizontal layers to become decoupled for lack of vertical turbulent mixing. The lack of strong turbulent mixing also provides the SBL with a rather long "memory" of events, especially related to terrain effects; hence the structure of the SBL is not solely dependent on local conditions. Patches of "fossil" turbulence can continue to exist quite removed from their locations of generation. Finally, because the SBL is not dominated by local forcing and turbulent mixing, as in the CBL, other processes can be equally important and require consideration. These include radiative heat transfer and interaction of internal gravity waves with turbulence.

The modeling difficulties associated with the SBL generally preclude use of sim-

ple approaches. The reduced vertical turbulent exchange means that the influence of surface conditions is limited to the layer near the ground. Thus the full vertical structure of the SBL is not related well to surface fluxes, as compared with the CBL; unfortunately, therefore, the information readily available near the surface is insufficient to determine SBL structure (Delage, 1974). Furthermore, the effectiveness of one-dimensional models for the CBL, where strong turbulent mixing dominates throughout, is not found for the SBL. The small vertical mixing and associated long diffusion times make horizontal advection an important process in the SBL (Nieuwstadt and Driedonks, 1979). Stable stratification tends to selectively damp vertical motions that have to work against the negative buoyancy. This selective damping 
results in large anisotropy that alters mechanisms associated with return to isotropy and energy cascade to smaller scales (Zeman and Lumley, 1979). Intermittency of turbulence in strongly stable conditions confounds traditional modeling approaches, such as Reynolds-averaged models. The lack of measurements, especially above the surface layer, compounds the problem and limits validation of alternative approaches such as stochastic models. Even where measurements are available, their use is limited by the low and variable levels of turbulence and the variability of mean conditions that hamper statistical analysis.

\subsection{Modeling Laboratory-Scale Flows}

Direct numerical simulation (DNS) has been used to study stably stratified flows on the laboratory scale. DNS resolves all the scales of motion, eliminating the need for a turbulence model. However, the domain is small due to computer limitations so DNS has been limited to rather low Reynolds number flows. Nonetheless, DNS results are like a numerical laboratory and provide a great deal of information that can be processed into diagnostic data for evaluating LES results.

\subsubsection{Direct Numerical Simulation}

Holt, et al. (1992) simulated stratified shear flows away from the wall. They found that flows could be divided into two categories: shear-dominated and buoyancy dominated. A transition Richardson number delineated the two flow categories. They observed that the critical Richardson number was an increasing function of the Reynolds number. This is contrary to later findings in the laboratory by Piccirillo and Van Atta (1997). Coleman, et al. (1992) addressed stratified shear flows that included the presence of a wall, and thus studied a laboratory-scale version of the atmospheric SBL. With some care, they extrapolated their results to higher Reynolds number, and compared them to atmospheric SBL studies of Nieuwstadt. As can be seen in Figure 4.5, the agreement is surprisingly good, especially when Nieuwstadt's data are filtered. This is an encouraging result since DNS provides detailed information about the flow without the use of any turbulence model, so the ability to extend DNS results to high 
Reynolds number benefits LES of the SBL.

Jabobitz, et al. (1997) used DNS to study the dependence of turbulence evolution on gradient Richardson number, Taylor microscale Reynolds number, and the initial value of the shear number. They confirmed the earlier finding of Holt, et al (1992) that the critical Richardson number does have a Reynolds number dependence at lower Reynolds numbers, but that the dependence of the growth rate parameter vanishes at $R e_{\lambda}>40$. The effect of initial shear number on the turbulence growth rate is non-monotonic (larger values of shear number do not necessarily lead to larger growth rates). Shih, et al. (2000) found that the stationary Richardson number depended on both the shear number and turbulent Reynolds number $\left(R_{e}\right)$ at low $R_{e}$, but depended only on $R_{e}$ at high $R_{e}$ (i.e. 89). Barnard (2000) studied the very stable boundary layer using DNS. The goal was to study the case where the turbulence was intermittent, rather than continuous. He found that a source of intermittent turbulence is a vigorous, inflectional (Ekman-like) instability (i.e. a roll cell) that lifts colder air over warmer air. This creates a convective instability that kicks off an intense burst of turbulence, which tends to destroy the lifting motion of the roll cell and the roll cell itself.

\subsubsection{Large-Eddy Simulation}

Kaltenbach, et al. (1994) performed LES of homogeneous stably stratified shear flows to study turbulent transport. They concluded that vertical overturning and mixing is suppressed when the inverse Froude number exceeds a critical value of 3. Garg, et al. (2000) used LES to study stably stratified channel flows. They identified three categories of flows: buoyancy affected, buoyancy controlled, and buoyancy dominated. These categories are defined by Richardson and Reynolds numbers. Buoyancy affected flows remain turbulent and achieve an equilibrium. Buoyancy controlled flows do not reach equilibrium, but rather develop in two parts where one part becomes inactive, and then is reactivated by the active half. The buoyancy dominated flow occur at higher Richardson numbers where the turbulence generation near the wall though the burst-sweep process is interrupted and ceases to produce turbulence. This leads to a relaminarization where the inner and outer layers become decoupled. 


\subsection{Modeling Atmospheric-Scale Flows}

\subsubsection{Structure of the evolving SBL}

The evolution of the SBL on a clear night is driven by a combination of surface cooling due to long-wave radiative flux divergence and a thickening of the cooled layer near the ground due to turbulent mixing. The growth rate of the SBL is related to the amount of shear production of turbulence, and is tempered by dissipation and buoyant destruction of turbulence.

Nieuwstadt and Tennekes (1981) developed a linear relaxation model of SBL height that incorporates the influence of initial conditions, a time-dependent relaxation limit, and time-history of the relaxation limit. The performance of their prognostic model was compared with that of the Zilitinkevich diagnostic model. As can be seen in Figure 4.1a, the diagnostic model underpredicted the observed $\mathrm{h}$, while the prognostic model (see Figure 4.1b) obtained much better agreement.

Stull (1983) developed a height scale based on the time history of surface heat flux; this approach had been suggested by others (Delage, 1974; Nieuwstadt, 1981). Stull's model predicts a $t^{1 / 2}$ dependence for the growth of the SBL height; this is consistent with observations and the results of Brost and Wyngaard (1978).

The amount of shear-driven turbulence near the ground is parameterized by the friction velocity $u_{*}$. The time history of this parameter should reveal the turbulent mixing available near the ground during SBL evolution, since the $u_{*}$ is directly related to the strength of the shear stress at the ground (and hence the generation of turbulence due to shear). Results of a second-order turbulence model (Wyngaard, 1975), shown in Figure 4.2a, reflect the rapid decay of turbulence in the first few hours of SBL development; observations support this finding. The sensitivity of this phenomena to the surface cooling rate is illustrated in Figure 4.2b. Increased cooling rates led to decreased steady-state values of $u_{*}$. Zeman (1979) developed a simple prognostic model for SBL height. This model suggests, as one would expect, that increased surface cooling leads to a more shallow SBL. Indeed, this is confirmed in the modeling results of Delage (1974), where a doubling of the cooling rate reduced the SBL height by as much as $80 \%$. 
As mentioned previously, the SBL has a rather long response time, so constant boundary conditions are needed for steady-state solutions. In the case of surface cooling, two options were exercised in the Brost and Wyngaard study (1978). The first, for steady-state runs, was a constant cooling rate, with the resulting temperature and heat flux profiles given in Figure 4.3a. After several hours, the cooling rate (heat flux divergence) has become constant with height. The second option was a surface energy budget that produced a higher cooling rate after sunset and eventually decreased later at night; obviously this led to a non-steady SBL The resulting profiles (see Figure 4.3b) show that the height of maximum cooling increases during the night. This, in turn, leads to a more uniform temperature distribution in the lower SBL with a steep gradient aloft. Such a temperature profile mimics an SBL with strong turbulent mixing near the surface.

The evolution of mean horizontal wind during the night, as predicted by a higherorder turbulence model of Andre, et al (1978), is shown in Figure 4.4a. The development of the low level jet at about $100 \mathrm{~m}$ is quite obvious, and is in qualitative agreement with the observations (in Figure 4.4b) except that the observed jet occurs at $200 \mathrm{~m}$. The modeled and observed evolution of the temperature profiles (Figures $4.4 \mathrm{c}, \mathrm{d}$ ) agree near the surface, but the observed profile indicates more cooling through a deeper layer above the ground after midnight. This could be due to advective effects that have been neglected in the model. Yamada and Mellor (1975) incorporated these effects to obtain better agreement with the data.

\subsubsection{LES of the SBL}

Mason and Derbyshire (1990) showed that LES of the SBL was feasible, and compared their results to SBL scaling studies of Nieuwstadt (1984b). However, the results were hampered by imperfections in the SGS model. Brown, et al. (1994) used a stochastic backscatter SGS model and repeated the earlier simulations of Mason and Derbyshire. The simulated SBLs were deeper and more turbulent than before. Their results for a range of surface buoyancy fluxes could be collapsed to single curves using local scaling.

Andren (1995) performed LES of the SBL using the Sullivan, et al. (1994) SGS 
model that has a two-part eddy viscosity. He found a two-part structure to the SBL, based on the distributions of vorticity, where the near surface part had typical shearflow structure, while the SBL then transitioned aloft into a structure typical of an internal wave field. Using quadrant analysis for the vertical fluxes, Andren found that a few intense bursts account for most of the flux and that this intermittency increases toward to SBL top.

Kosović and Curry (2000) used LES to study SBLs typical of those observed in the Arctic. A nonlinear SGS stress model (Kosović, 1997) is used that accounts for backscatter and shear effects. They simulated a range of geostrophic winds, surface cooling, surface roughness, overlying inversion strengths, and latitude (to test Coriolis effects). Their simulated SBLs agreed with observations, showing the typical two-layer boundary layer structure, elevated inversions, and low level jets.

Saiki, et al. (2000) studied a windy SBL (15 m/s geostrophic wind) with strong surface cooling $(-0.05 \mathrm{~K} \mathrm{~m} / \mathrm{s})$. The simulated SBL had continuous turbulence with small scale structures. A strong low level jet formed just above the top of the SBL. A strong capping inversion was specified and this, along with the underlying SBL, led to the development of a dominant gravity wave at the top of the SBL which appeared to be related to the most unstable wave predicted by the Taylor-Goldstein equation. 


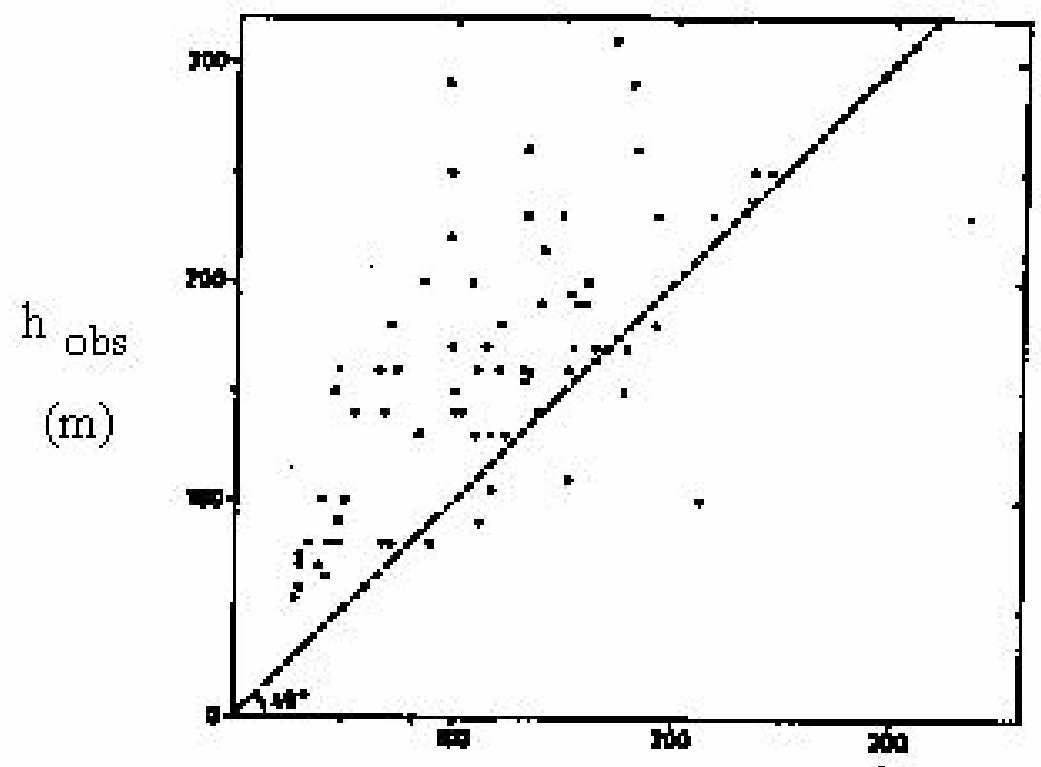

(a)

$\mathrm{h}_{\text {diagnostic model }(\mathrm{m})}$

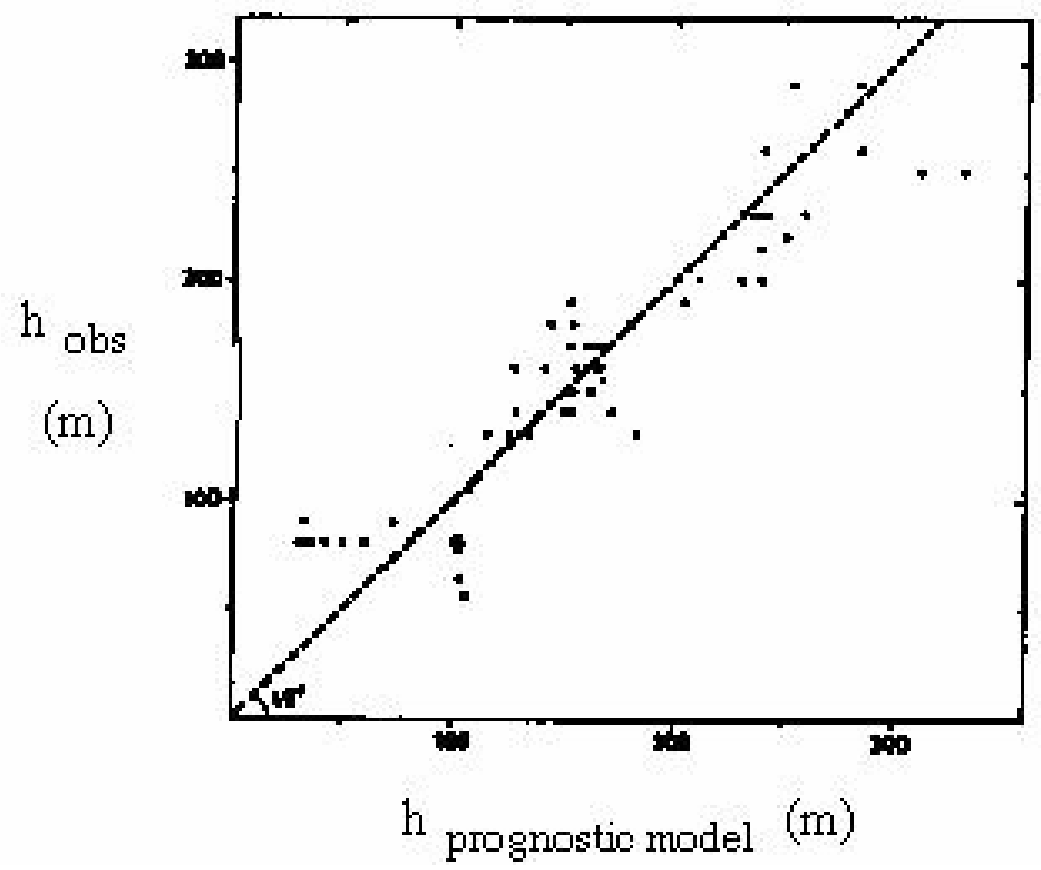

(b)

Figure 4.1: Comparison of observed SBL heights with values calculated by (a) diagnostic model of Zilitinkevich (1972), $h=0.4 \sqrt{u_{*} L / f}$, and (b) prognostic model of Nieuwstadt and Tennekes (1981). 


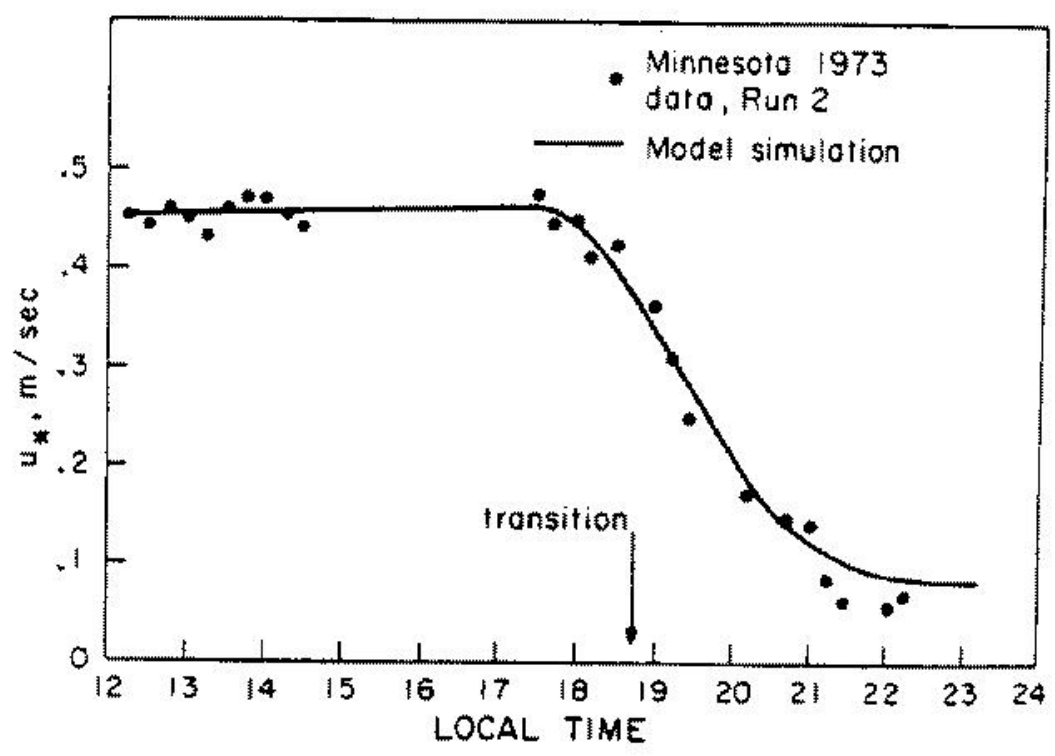

(a)

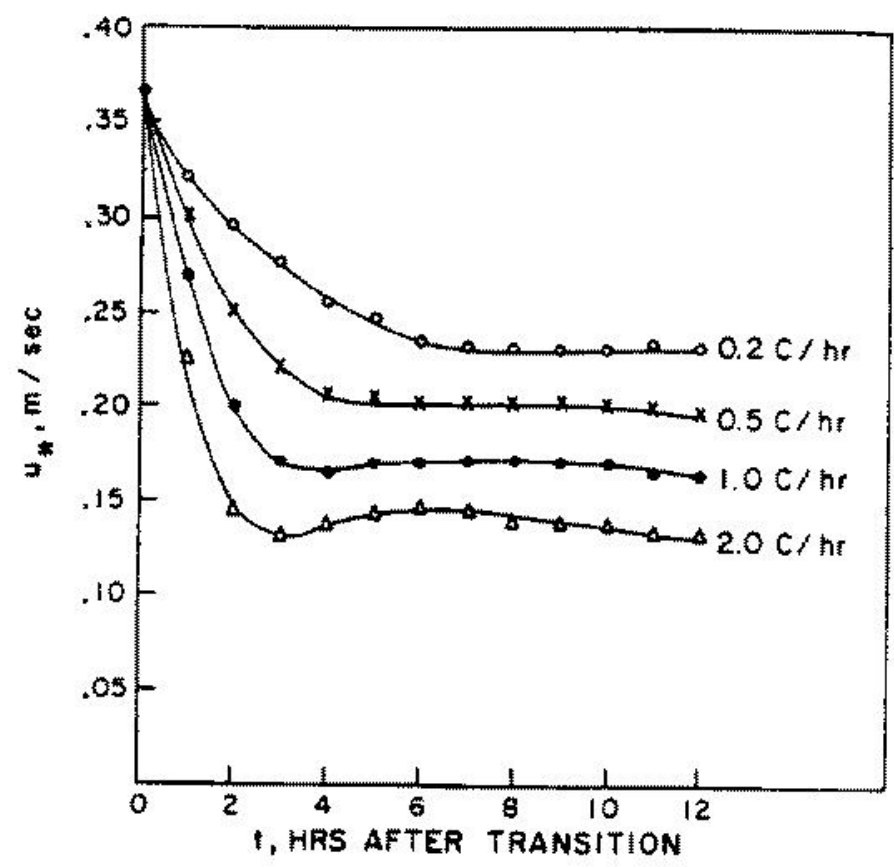

(b)

Figure 4.2: Evolution of friction velocity $u_{*}$ for (a) day to night periods and (b) modeled periods at night with various surface cooling rates (after Wyngaard, 1975). 
(a)

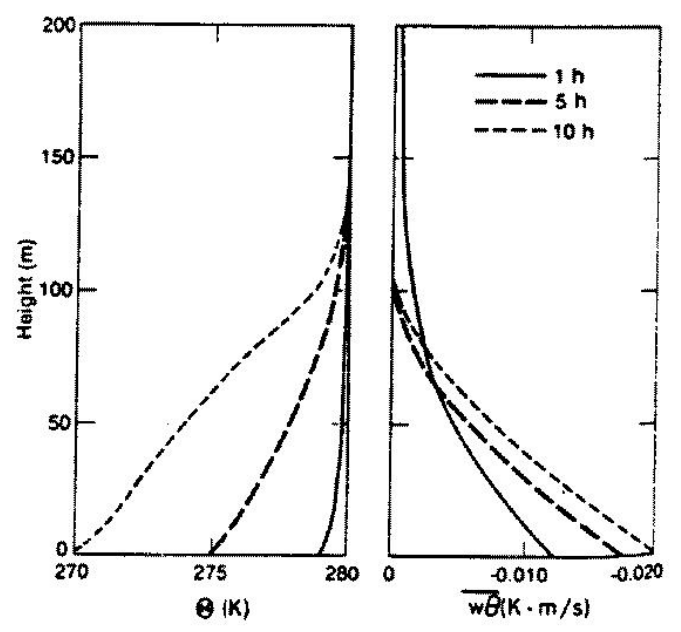

(b)

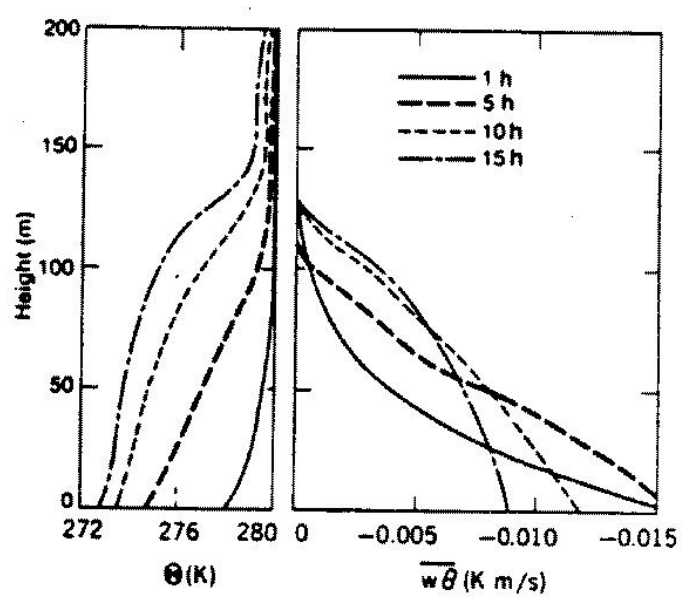

Figure 4.3: Calculated time evolution of potential temperature and vertical potential temperature flux for (a) constant cooling rate at $1 \mathrm{~m}$ of $1 \mathrm{~K} / \mathrm{hr}$, and (b) cooling rate from surface energy budget (after Brost and Wyngaard, 1978). 

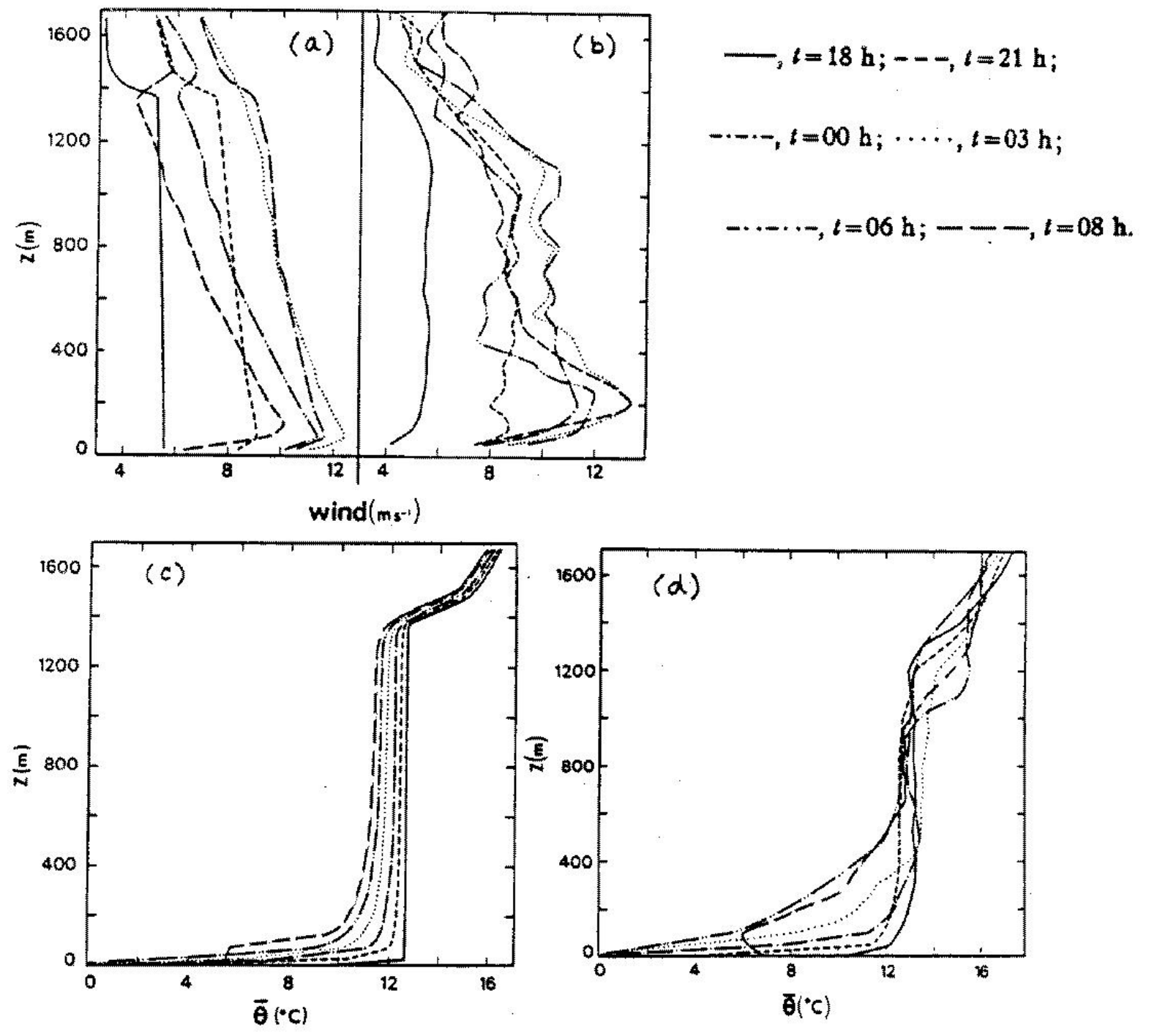

Figure 4.4: Profiles of wind speed (a) computed and (b) observed, and potential temperature (c) computed and (d) observed for Wangara Night 33-34 for times indicated (after Andre, et al., 1978). 

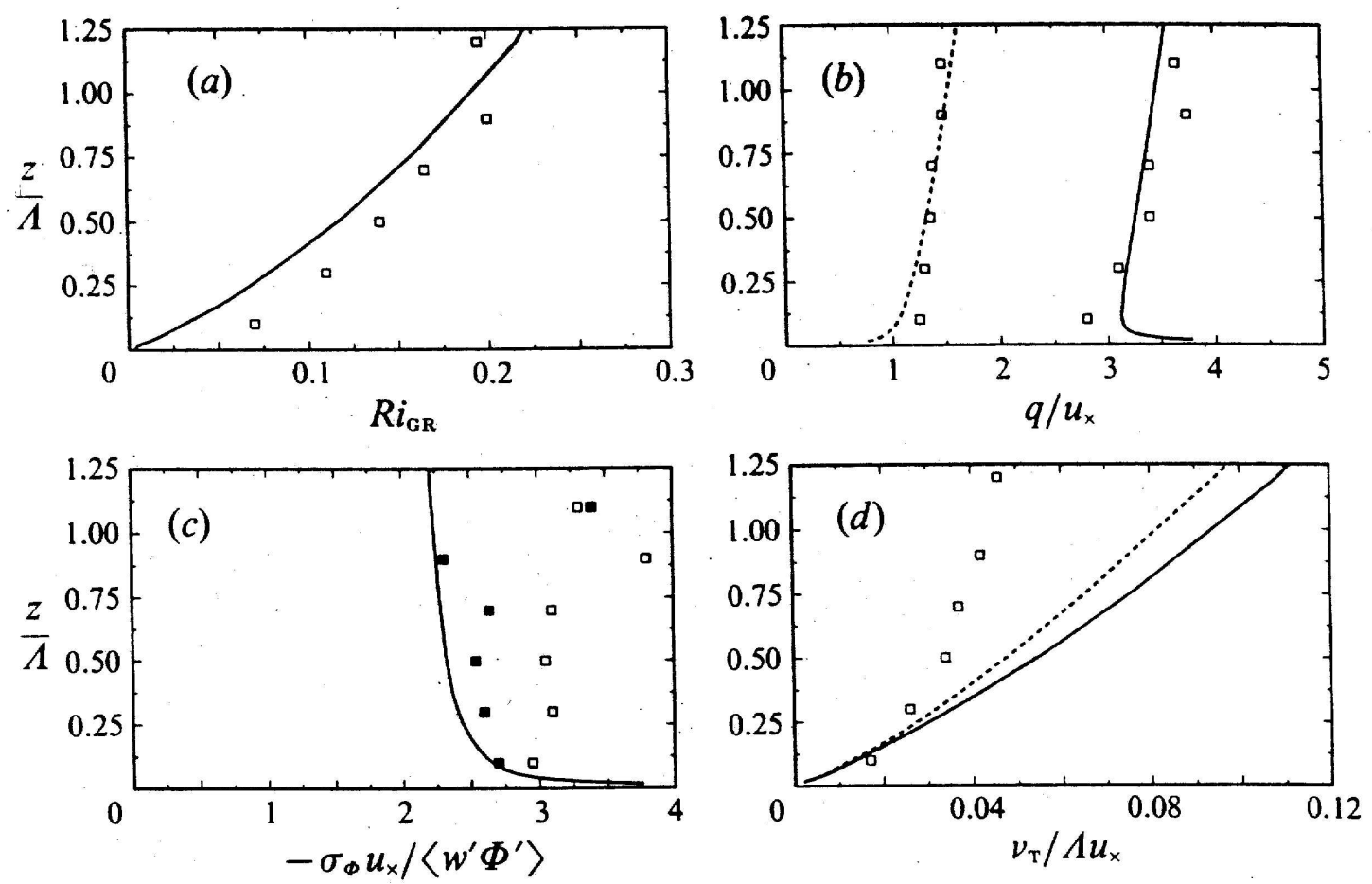

Figure 4.5: Comparison of Coleman, et al. DNS results with Nieuwstadt atmospheric SBL data for (a) gradient Richardson number; (b) RMS velocity fluctuations for total (solid) and vertical component (dashed); (c) RMS temperature fluctuations; and (d) eddy viscosity, where solid and dashed lines are different eddy viscosity formulations; Nieuwstadt (1984b) data unfiltered (open squares) and filtered (solid squares); (after Coleman, et al., 1992). 


\section{Chapter 5}

\section{Numerical Formulation}

From the preceding chapters, two points emerge that are relevant to modeling the evolving SBL: (1) the SBL is non-steady with turbulence that is not characterized well by traditional Reynolds-averaging closure, and (2) LES is well suited to treating turbulence that is complicated at scales that can be resolved by a numerical model. For simulating the time- and space-varying levels of turbulence that occur at night in the SBL, LES requires the fewest assumptions about the behavior of turbulence across a wide range of scales. The greatest difficulty lies in choosing an adequate subgrid-scale (SGS) turbulence model. In spite of this, LES offers the best approach for simulating the evolving SBL.

The LES model used as a starting point for this research is that by Brost (Wyngaard and Brost, 1984; Nieuwstadt and Brost, 1986). The Brost LES model is a derivative of a dry version of Deardorff's LES model (Deardorff, 1980). It is designed to address the clear-sky, CBL under horizontally homogeneous conditions. It is extended to the SBL in this work.

\section{$5.1 \quad$ Model Equations}

\subsubsection{Governing Equations}

Governing equations are formed for the volume-averaged velocity, and potential temperature $\bar{\theta}$, which is defined as

$$
\bar{\theta}=T\left(p_{0} / p\right)^{R / c_{p}}
$$

where $T$ is air temperature $(\mathrm{K}), p_{0}$ is a reference pressure $(100 \mathrm{kPa}), p$ is the barometric pressure, $\mathrm{R}$ is the gas constant, and $c_{p}$ is the specific heat such that $R / c_{p}=0.286$. 
The equations of motion for this incompressible Boussinesq flow, with the momentum equations in flux form, are:

$$
\begin{gathered}
\partial \bar{u}_{i} / \partial x_{i}=0 \\
\frac{\partial \bar{u}_{i}}{\partial t}=-\frac{\partial \bar{u}_{i} \bar{u}_{j}}{\partial x_{j}}-\frac{\partial \bar{p} / \bar{\rho}_{0}}{\partial x_{i}}-\frac{\partial P}{\partial x_{i}}+2 \epsilon_{i j 3} \Omega_{j} \bar{u}_{j}+\frac{g}{\bar{\theta}_{0}}(\bar{\theta}-\langle\bar{\theta}\rangle)-\frac{\partial \tau_{i j}}{\partial x_{j}}-\nabla^{4} \bar{u}_{i}
\end{gathered}
$$

where $\bar{u}_{i}$, (i=1,2,3 and also noted $\left.\bar{u}, \bar{v}, \bar{w}\right)$ are the resolved-scale velocity components in the eastward, northward, and upward directions, respectively, corresponding to the positive $\mathrm{x}, \mathrm{y}$, and $\mathrm{z}$ axes $\left(x_{i}, i=1,2,3\right)$; the coordinate scheme is illustrated in Figure 5.1. The horizontal averages are denoted by \langle\rangle$. \mathrm{P}$ is the large-scale pressure, whose horizontal gradient is related to the geostrophic forcing by $\partial P / \partial x=f V_{g}$ and $\partial P / \partial y=-f U_{g}$, where $f$ is the Coriolis parameter. $\bar{p}$ is the deviation from horizontal mean pressure. $\Omega_{j}$ is the component of the earth's rotation. $\tau_{i j}$ is the SGS stress tensor, to be defined in the next subsection, and $\nabla^{4} \bar{u}_{i}$ is a 4 th order dissipation term added to control nonlinear instabilities. Both $\tau_{i j}$ and $\nabla^{4} \bar{u}_{i}$ act to transfer energy near the filter scale; see Collis (2001) for further discussion of multiscale aspects of energy transfer in LES. The $\nabla^{4} \bar{u}_{i}$ term is discussed further in Section 5.2. Molecular dissipation is not considered since it is several orders of magnitude smaller than that due to atmospheric turbulence.

The governing equation for the potential temperature is

$$
\frac{\partial \bar{\theta}}{\partial t}=-\frac{\partial \bar{u}_{i} \bar{\theta}}{\partial x_{i}}-\frac{\partial \chi_{k}}{\partial x_{k}}-\nabla^{4} \bar{\theta}
$$

$\chi_{k}$ is the SGS heat flux, discussed further in the next subsection. $\nabla^{4} \bar{\theta}$ is a 4 th order dissipation term similar to that used in Equation 5.3 and is discussed further in Section 5.2. 


\subsubsection{SGS Turbulence Model}

Two extra terms which represent the effect of the subgrid-scale motion appear in the governing equations, $\tau_{i j}$ and $\chi_{j}$, are defined as

$$
\begin{aligned}
\tau_{i j} & =\bar{u}_{i} u_{j}-\bar{u}_{i} \bar{u}_{j}, \\
\chi_{j} & =\overline{u_{j} T}-\bar{u}_{j} \bar{T} .
\end{aligned}
$$

These terms cannot be calculated explicitly and thus must be modeled; in the approach used here, the modeling is in terms of known resolved-scale quantities.

The SGS turbulence model used is based on the dynamic mixed model of Zang, et al. (1993), and is extended to treat potential temperature. Models for $\tau_{i j}$ and $\chi_{j}$ take the following form

$$
\begin{aligned}
\tau_{i j}-\frac{\delta_{i j}}{3} \tau_{k k} & =-2 C_{1} \nu_{T} \bar{S}_{i j}+C_{2}\left(L_{i j}^{m}-\frac{\delta_{i j}}{3} L_{k k}^{m}\right), \\
\chi_{k} & =-C_{3} \kappa_{T} \frac{\partial \bar{\theta}}{\partial x_{k}}+C_{4} P_{k}
\end{aligned}
$$

where $\bar{S}_{i j}$ is the resolved strain rate tensor,

$$
\bar{S}_{i j}=\frac{1}{2}\left(\frac{\partial \bar{u}_{i}}{\partial x_{j}}+\frac{\partial \bar{u}_{j}}{\partial x_{i}}\right)
$$

$L_{i j}^{m}$, the 'modified Leonard term' (Germano, 1986), and $P_{j}$ are defined as

$$
\begin{aligned}
L_{i j}^{m} & =\overline{\bar{u}}_{i} \bar{u}_{j}-\overline{\bar{u}}_{i} \overline{\bar{u}}_{j}, \\
P_{k} & =\overline{\bar{u}}_{k} \bar{\theta}-\overline{\bar{u}}_{k} \overline{\bar{\theta}} .
\end{aligned}
$$

The values of $C_{1}$ and $C_{3}$ are determined dynamically. The values of $C_{2}$ and $C_{4}$ can take the value 0 or 1 , or may be determined dynamically depending on the type of subgrid-scale model being used. For the model used here, $C_{2}$ and $C_{4}$ are given values of 1 ; this is consistent with the Taylor series expansion where the coefficient for these terms is 1 (cf., Katapodes, et al., 2000). $\nu_{T}$ and $\kappa_{T}$ are called the 'eddy viscosity' and the 'eddy diffusivity', respectively. The isotropic part involving $\tau_{k k}$ and $L_{k k}$ is to be lumped into the pressure gradient term. 
Using the Smagorinsky dissipation kernel, we can write the SGS terms as

$$
\begin{aligned}
\tau_{i j}-\frac{\delta_{i j}}{3} \tau_{k k} & =-2 C_{1} \Delta^{2}|\bar{S}| \bar{S}_{i j}+\left(L_{i j}^{m}-\frac{\delta_{i j}}{3} L_{k k}^{m}\right), \\
\chi_{j} & =-C_{3} \Delta^{2}|\bar{S}| \frac{\partial \bar{\theta}}{\partial x_{k}}+P_{k} .
\end{aligned}
$$

An option was developed, but not used, that incorporates a dissipation kernel based on the Deardorff (1980) SGS model that calculates a prognostic SGS TKE to obtain a SGS velocity and provides a length scale related to the grid resolution. A version of the SGS model was developed for dynamically calculating $C_{2}$ and $C_{4}$. Also, options were developed for various averagings of the dynamic coefficient, including the techniques used in Zang, et al. (1993) following Lilly (1992). However, the dynamic coefficients calculated for the high Reynolds SBL were quite variable. In the end, the most robust approach was that of Germano, et al. (1991) where the values obtained dynamically for $C_{1}$ and $C_{3}$ are averaged across the horizontal plane and allowed to vary in the vertical and in time. The dissipative terms that employ $C_{1}$ and $C_{3}$ are not critical to horizontal variability in the SGS model, since most of the SGS stress and flux is carried in the second terms on the RHS of Equations 5.12 and 5.13 that allow variability in all three spatial dimensions and in time.

\subsection{The Grid and Numerical Techniques}

Before discussing the bottom boundary conditions used in this model, it is useful to document the staggered grid used in the model. The grid arrangement is an Arakawa C-grid and is similar to that used by Nieuwstadt and by Schumann in a comparison of four LES codes (Andren, et al., 1994). The staggering of the velocity and stress components, SGS TKE, pressure, and temperature is illustrated in Figure 5.2. The staggering of the velocity components facilitates the calculation of velocity gradients required by the strain rates used in the dynamic SGS model.

Spatial derivatives are calculated using a centered difference technique, which is second-order accurate. For the non-linear advection terms, we use the method of Piacsek and Williams (1970) which conserves variances. This scheme is equivalent to that of Lilly (1965) when the assumption of continuity is met. The method is defined 
as

$$
\frac{\partial u_{i} \phi_{i}}{\partial x_{i}} \equiv\left(u_{i+1 / 2} \phi_{i+1}-u_{i-1 / 2} \phi_{i-1}\right) / 2 \Delta x
$$

The method is illustrated in Figure 5.3. The pressure is solved using a direct solver (Kau and Peskin, 1972; Deardorff, 1973). With periodic horizontal boundary conditions, a Fourier solution is performed in the horizontal directions at each level. Then a matrix inversion is performed in the vertical direction, with gradient boundary conditions applied at the top and bottom.

The equations are integrated in time using a leapfrog scheme, which is secondorder accurate and non-dissipative. To control for the computational mode, a time filter was used that was developed by Robert (1966) and investigated further for threedimensional atmospheric flows by Klemp and Wilhelmson (1978). The technique uses the following algorithm to advance the value of a variable $\phi$ at each time step from rate of change information $\partial \phi / \partial t=F$ :

$$
\begin{aligned}
\phi^{* t+\Delta t} & =\phi^{t-\Delta t}+2 \Delta t F^{* t} \\
\phi^{t} & =\phi^{* t}+\alpha\left(\phi^{* t+\Delta t}-2 \phi^{* t}+\phi^{t-\Delta t}\right)
\end{aligned}
$$

The first step of the algorithm is the leapfrog step with the asterisk denoting terms that have not been smoothed yet by the second step. Asselin (1972) evaluated the damping characteristics and found that the computational modes were effectively damped with little effect on the physical modes. To minimize the effects on the small resolved scales used in the SGS model, the damping factor is reduced from $\alpha=0.1$ originally used by Brost and Wyngaard (1984) in the 'starting point' version of the LES model to a value of $\alpha=0.02$ for integrating the momentum equations and $\alpha=0.06$ for the potential temperature equation.

Since there is minimal numerical diffusion in the methods used, a 4th order dissipation term is included in the governing equations to control for non-linear instabilities. This technique has proved useful for flows with sharp gradients (for example, see MacCormack and Baldwin, 1975). The effect in atmospheric flows has been illustrated by Pielke (1984) and included here in Figure 5.4. Waves smaller than $2 \Delta x$ (associated with the Nyquist or folding frequency) are seen as larger waves on the 
computational grid (folded over to lower frequencies by aliasing). The term is of the form $\nabla^{4} \phi=K_{D}\left(\delta_{x x x x} \phi\right)$, where $\delta_{x x x x}$ is the 4 th-order differential operator (see Fletcher, 1991, vol. 2, p445, eq 18.156). The value of $K_{D}$ is set to a small enough value so that the important scales of the simulation are not affected, and yet large enough to control for any erroneous energy buildup near $2 \Delta x$. In their simulations, Klemp and Wilhelmson (1978) used a value of $K_{D} \Delta t / \Delta x^{4}=0.0025$ with satisfactory results. For the simulations presented here, a smaller value is used to reduce any undesirable effects on the small scales used by the SGS model: $K_{D} \Delta t / \Delta x^{4}=0.001$.

\subsection{Boundary Conditions}

\subsubsection{Upper and Lateral Boundary Conditions}

At the top of the model domain, the momentum forcing is a prescribed, constant geostrophic wind. The vertical motion is zero, and the vertical temperature gradient is prescribed. The lateral boundary conditions are periodic. This is required by the pressure solver. Although use of periodic boundary conditions limits the application to horizontally homogeneous situations, much can be learned about the evolution of SBL structure in the vertical.

Rayleigh damping is used in the upper third of the model domain to absorb vertically propagating waves and eliminate reflection at the top boundary. The damping term is added to the right-hand side of the governing equations, in the form of 'relaxation' back to a specified value. The relaxation time scale for the damping $R_{D}$ follows Klemp and Lilly (1978)

$$
R_{D}=\alpha_{R}\left\{1-\cos \left[\pi\left(z-z_{B}\right) /\left(z_{T}-z_{B}\right)\right]\right\} / 2
$$

where $z_{B}$ is the bottom of the damping (or sponge) layer and $z_{T}$ is the top of the model. $\alpha_{R}$ is the e-folding time scale at the model top. A value associated with 10 50 time steps is recommended; a value related to 10 time steps is used here to insure that wave-like motions in the simulations are not due to unwanted reflections from the upper boundary. 


\subsubsection{Initial Conditions}

The model is initialized with an Ekman spiral consistent with the geostrophic forcing and surface roughness, following Appleby and Ohmstede (1964). The initial wind profiles are obtained by assuming a balance between the large-scale horizontal pressure gradient and the Coriolis force, so that the winds at the top of the boundary layer are geostrophic. The surface is assumed to be horizontally homogeneous and characterized by a roughness length $z_{0}$. The equations of motion for this simplified flow are:

$$
\begin{aligned}
-f\left(V-V_{g}\right) & =\frac{\partial(-\overline{u w})}{\partial z} \\
f\left(U-U_{g}\right) & =\frac{\partial(-\overline{v w})}{\partial z}
\end{aligned}
$$

The bottom boundary conditions for the stresses are specified in terms of a friction velocity $u_{*}$. Thus for a specified geostrophic wind, surface roughness and associated friction velocity, solutions to Equations 5.18 and 5.19 provide an initial wind field. This wind field is then perturbed by fluctuations that are a scaled combination of sine and cosine terms.

The initial potential temperature profile is specified as constant from the surface up to some height $\mathrm{H}$, with a small, specified positive lapse rate above that. For most of the simulations, the height $\mathrm{H}$ is about half the domain height.

\subsubsection{Lower Boundary Conditions}

The rigorous boundary condition for velocity at the ground would be a no-slip condition. However, for the atmospheric boundary layer, the ground is aerodynamically rough and the small scales of the flow are not resolved. It is well-documented, on the other hand, that the shear present in the layer near the wall is an important source of turbulence. This must be taken into account when prescribing the bottom boundary condition for velocity and/or stress. At high Reynolds number, as found in the PBL, it is not practical to resolve the details of turbulence-generating flow that account for the wall stress. The thickness of the viscous sublayer is about a millimeter, which is several orders of magnitude smaller than the depth of the PBL. 
An alternative approach is to specify the wall stress in terms of calculated information in the region removed the wall where the velocity profile is approximately logarithmic, following the law of the wall. In this region, the stress is nearly constant with height. Hence, a reasonable estimate of the wall stress can be made by approximating the stress using the local velocity field. The vertical components of the stress tensor to be estimated are $\overline{u^{\prime} w^{\prime}}$ and $\overline{v^{\prime} w^{\prime}}$. The vertical component of the heat flux is $\overline{w^{\prime} \theta^{\prime}}$.

There are several approaches that have been taken for specifying this alternative boundary condition, as reviewed by Piomelli, et al. (1989). Schumann (1975) required a priori knowledge of the driving pressure gradient to calculate the horizontal average of the wall stress. Grötzbach (1986) took a different approach where the horizontallyaveraged wall stress was obtained from the velocity in the logarithmic layer. In this way, he extended the applicability of Schumann's method by relaxing the requirement of it a priori knowledge of the pressure gradient. The modified approach also allowed local variation of the total mass flux through the channel.

The approach used here follows Deardorff (1974a), in his simulation of the atmospheric boundary layer. Horizontal velocities at the lowest computational level are used:

$$
\begin{aligned}
& \overline{u^{\prime} w_{0}^{\prime}}=-C_{D} S \bar{u}_{\Delta z / 2} \\
& \overline{v^{\prime} w^{\prime}}=-C_{D} S \bar{v}_{\Delta z / 2}
\end{aligned}
$$

The wind speed $\mathrm{S}$ is obtained from the $\mathrm{u}$ and $\mathrm{v}$ components at $z=\Delta z / 2$ in the usual way:

$$
S=\sqrt{\bar{u}^{2}+\bar{v}^{2}}
$$

The drag coefficient $C_{D}$ is obtained from wind velocities at $z=\Delta z / 2$, and the surface roughness $z_{0}$, and an assumed logarithmic velocity profile, by:

$$
C_{D}=k^{2} / \ln \left(z / z_{0}\right)^{2}
$$

where $z_{0}=0.1 \mathrm{~m}$ is used in this study and is representative of flat pasture land. Once 
the surface stresses are known, then the friction velocity is calculated as

$$
u_{*}=\left({\overline{u^{\prime} w_{0}^{\prime}}}^{2}+{\overline{u^{\prime} w_{0}^{\prime}}}^{2}\right)^{0.25}
$$

Similar alternative approaches exist, especially if the friction velocity is specified. For example, Mason and Thomson (1987) defined the wall stresses by:

$$
\begin{aligned}
& \overline{u^{\prime} w_{0}^{\prime}}=-u^{* 2} \cos \phi \\
& \overline{u^{\prime} w^{\prime}}==-u^{* 2} \sin \phi
\end{aligned}
$$

where $\phi=\tan ^{-1}(\bar{v} / \bar{u})$. Moeng (1984) used the local wind field to modify a horizontallyaveraged stress:

$$
\begin{aligned}
{\overline{u^{\prime} w^{\prime}}}_{0} & =\frac{\left\langle{\overline{u^{\prime} w_{0}^{\prime}}}_{0} S\left\langle\bar{u}_{\Delta z / 2}\right\rangle+\langle S\rangle\left(\bar{u}_{\Delta z / 2}-\left\langle\bar{u}_{\Delta z / 2}\right\rangle\right)\right.}{\langle S\rangle \bar{u}_{\Delta z / 2}} \\
{\overline{v^{\prime} w^{\prime}}}_{0} & =\frac{\left\langle{\overline{v^{\prime} w_{0}^{\prime}}}_{0}\right\rangle\left\langle\bar{v}_{\Delta z / 2}\right\rangle+\langle S\rangle\left(\bar{v}_{\Delta z / 2}-\left\langle\bar{v}_{\Delta z / 2}\right\rangle\right)}{\langle S\rangle \bar{v}_{\Delta z / 2}}
\end{aligned}
$$

The above approach allows local variation of the stress, analogous to the local variation in the Grötzbach (1986) approach.

The treatment of SGS turbulent transfer near the surface (a rough wall in this case) presents difficulty for many SGS formulations. The resolved scale stress decreases rapidly as the surface is approached, and while the SGS stress increases as the wall is approached, the increase is often not rapid enough, leaving a 'notch' in the total stress profile (see Figure 5.5a). Sullivan, et al. (1994) proposed breaking the eddy viscosity into two parts to capture better the turbulent transfer near the surface, following the work of Schumann (1975). This effort has been extended by Ding, et al. (2001), but both prescriptions are rather ad hoc.

A more well-founded approach has been proposed by Brown, et al. (2001). They employ a 'canopy' layer to represent the additional SGS turbulent transfer that has been missing near the surface. In this approach, an additional stress term, based on the wind speed, a drag coefficient, and a canopy density function $a$, is introduced to 
the governing equations for velocity:

$$
\left(\frac{\partial u_{i}}{\partial t}\right)_{c a n o p y}=-C_{D} a|u| u_{i}
$$

Brown, et al. (2001) set the value of $C_{D}$ to 0.1 empirically to match wind tunnel data. Equation 5.29 can be rewritten as

$$
\frac{\partial \tau_{\text {canopy }}}{\partial z}=-C_{D} a|u| u_{i}
$$

or

$$
\tau_{\text {canopy }}=-\int C_{D} a|u| u_{i} \partial z
$$

where $\tau_{\text {canopy }}$ is referred to as the 'canopy stress' and the constant of integration is set such that $\tau_{\text {canopy }}=0$ at the top of the canopy. The canopy density function $a$ is the leaf area per unit volume and has units of $m^{-1}$. It is defined for heights below the top of the canopy $\left(z<h_{c}\right)$ by

$$
a=a_{0} \cos ^{3}\left[\left(\pi z / 2 h_{c}\right)\right]
$$

At or above the top of the canopy, $a=0$. For this implementation of the approach, the value of $a_{0}$ was set such that the value of the total stress at the first computational level $(z=\Delta z / 2)$ is equal to the local $u_{*}^{2}$. The use of the canopy stress eliminated the necessity of calculating the vertical stress terms dynamically at the lowest computational level in the model. The canopy height $h_{c}$ is set equal to the horizontal grid spacing, which is taken to represent the scale of near surface eddies. Note that in Brown, et al. (2001), the cosine term in Equation 5.32 is raised to the power of two. The purpose of this term is to provide a smooth enough transition near the top of the canopy to avoid numerical noise associated with a sharp cutoff when $a$ is constant through the canopy and abruptly drops to zero. In the numerics used here, a sharper cutoff could be used without numerical noise and resulted in a more desirable stress profile, where the 'notch' is eliminated (see Figure 5.5b). In tests with varying grid resolutions, it was found that setting $h_{c}$ to the horizontal grid resolution did not work well when the grid resolution was the same in all three directions. A more robust 
choice is to set $h_{c}=2.5 \Delta z$. The combination of the resolved, SGS, and canopy terms blend into a smooth profile, as shown in Figure 5.6; note that the height scale is logarithmic in this figure.

The rationale of the canopy model and other approaches noted above has an interpretation in the representation of the flow on a grid. Far from the wall, the filtered flow represents most of the flow energy with the so-called subgrid-scale terms carrying the energy held on the grid beyond the resolved scales. As the wall is approached, two things happen. First, more and more of the energy is neither resolved nor held on the grid and so is truly 'subgrid' and not represented. Second, due to the typical grid anisotropy, $\Delta z \ll \Delta x$ and $\Delta y$; thus, near the wall, eddies smaller than $\Delta x$ in diameter are not properly represented. The canopy model (and all other ad hoc models) fill this gap in representation by assuring proper stress behavior near the wall.

The nondimensional wind shear $\phi_{m}$ has been used in a previous study to compare LES models for the neutral atmospheric boundary layer (Andren, et al., 1994). Values for $\phi_{m}$ can be obtained from LES-generated wind profiles using the definition of $\phi_{m}$

$$
\phi_{m}=\frac{k z}{u_{*}} \frac{\partial S}{\partial z}
$$

where $\mathrm{S}$ is wind speed defined in Equation 5.22. The vertical profile of $\phi_{m}$ obtained from LES results for a 1-hour simulation of the neutral boundary layer (NBL) is shown in Figure 5.7, along with the curve for $\phi_{m}=1+z / L$ (Dyer, 1972). Away from the surface, the profile for $\phi_{m}$ for our LES results is superior to all the models compared in Andren, et al. (1994) except the one LES model that used a backscatter SGS model. Near the surface, the values of $\phi_{m}$ for our LES results approach 0.5, compared to a value of 1 approached by the other LES models. We attribute this to the reduced vertical gradient of wind speed caused by the 'canopy' stress term. Within real canopy layers, the vertical gradient of wind speed tends to be very small.

Finally, it is the case that the choice of approach for specifying the bottom boundary condition for velocity is not critical in these simulations. The resulting effects are seen mostly in the stress profile near the ground. The differences are more cosmetic than substantive in terms of the resulting mean flow and turbulence. In addition, as 
will be clear from the simulation results, the most interesting features of the evolving SBL occur away from the surface and hence are not closely tied to near-surface features related to the bottom boundary condition.

The bottom boundary condition for the temperature equation is a specified surface heat flux. An alternative would be a specified surface temperature or temperature tendency. For all the runs, except for the CBL runs G10.4wt-02T and G10.4wt-04T, the specified heat flux is zero for the first hour. The heat flux is decreased linearly over the next hour to the SBL target value, which is maintained for the remainder of the simulation. For the CBL runs, the surface heat flux is zero for the first hour, increased linearly to the CBL value over the next hour (in this case $0.06 \mathrm{~K} \mathrm{~m} / \mathrm{s}$ ), held constant for an hour, then decreased linearly for an hour to the target SBL value, and held constant for the remainder of the simulation. The canopy model described above is applied to the heat flux near the wall. The implementation is the same as that for the stress, except that the 'canopy heat flux' at the lowest computational level is matched to the local surface heat flux. 


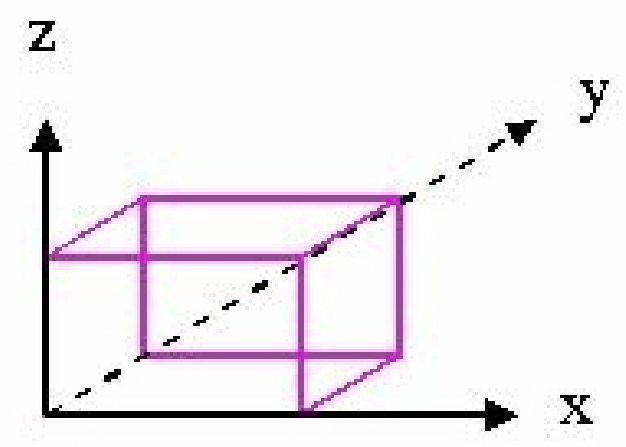

Figure 5.1: Coordinate system, where $\mathrm{x}, \mathrm{y}, \mathrm{z}$ may also be referred to as $x_{1} x_{2} x_{3}$, respectively; $\mathrm{x}$-axis is aligned with the mean wind near the surface.

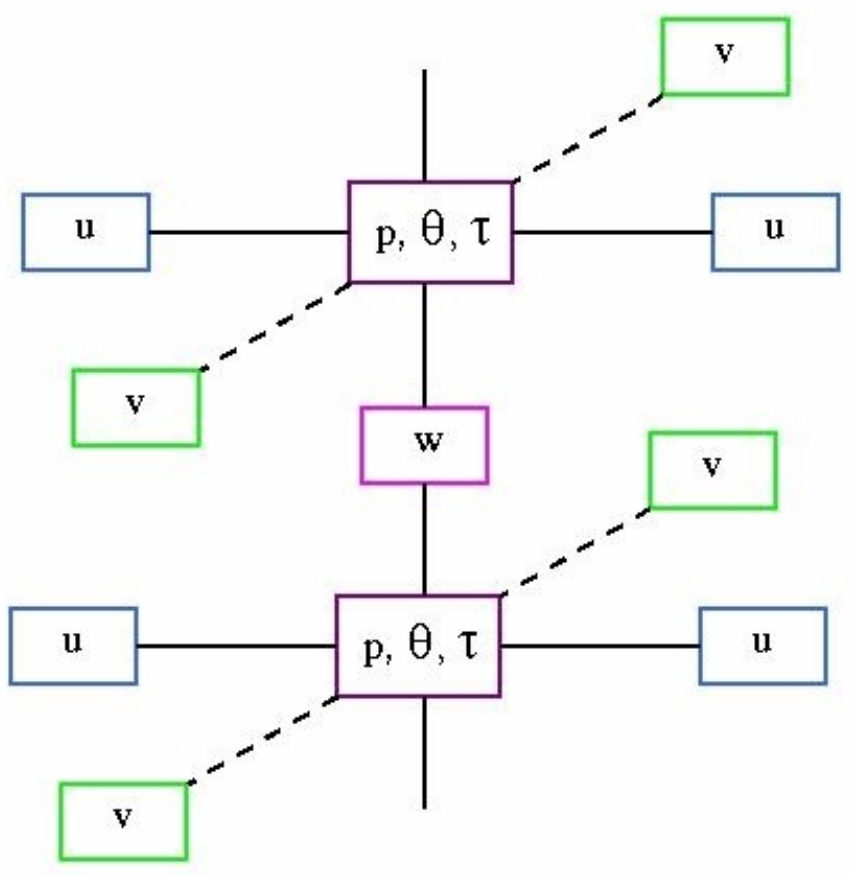

Figure 5.2: Arakawa-C grid with staggered assignments for velocity, where u, v, w may also be referred to as $u_{1} u_{2} u_{3}$, respectively. 


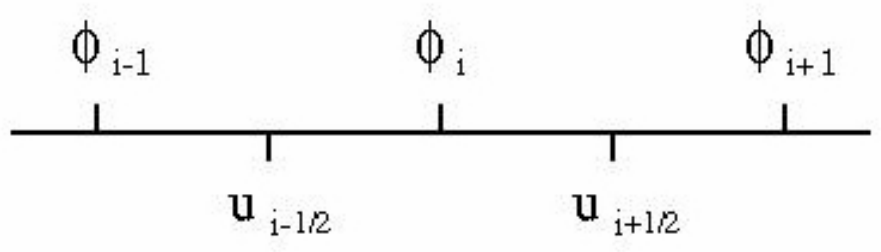

Figure 5.3: Advection scheme of Piacsek and Williams.

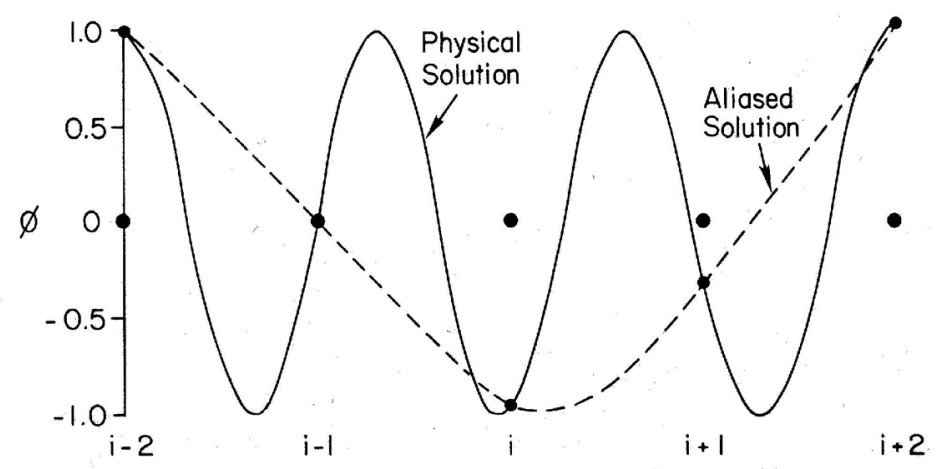

Figure 5.4: Schematic illustration of aliasing effects where physical solution of $1.33 \Delta x$ wavelength is seen as $4 \Delta x$ wave on computational grid due to interaction of $4 \Delta x$ and 2 $\Delta x$ waves (after Pielke, 1984). 


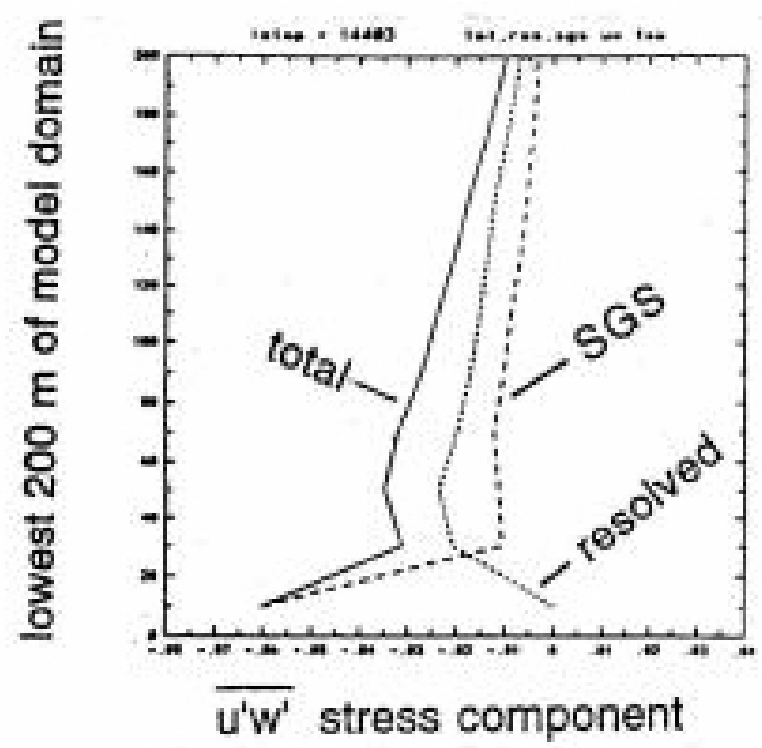

(a)

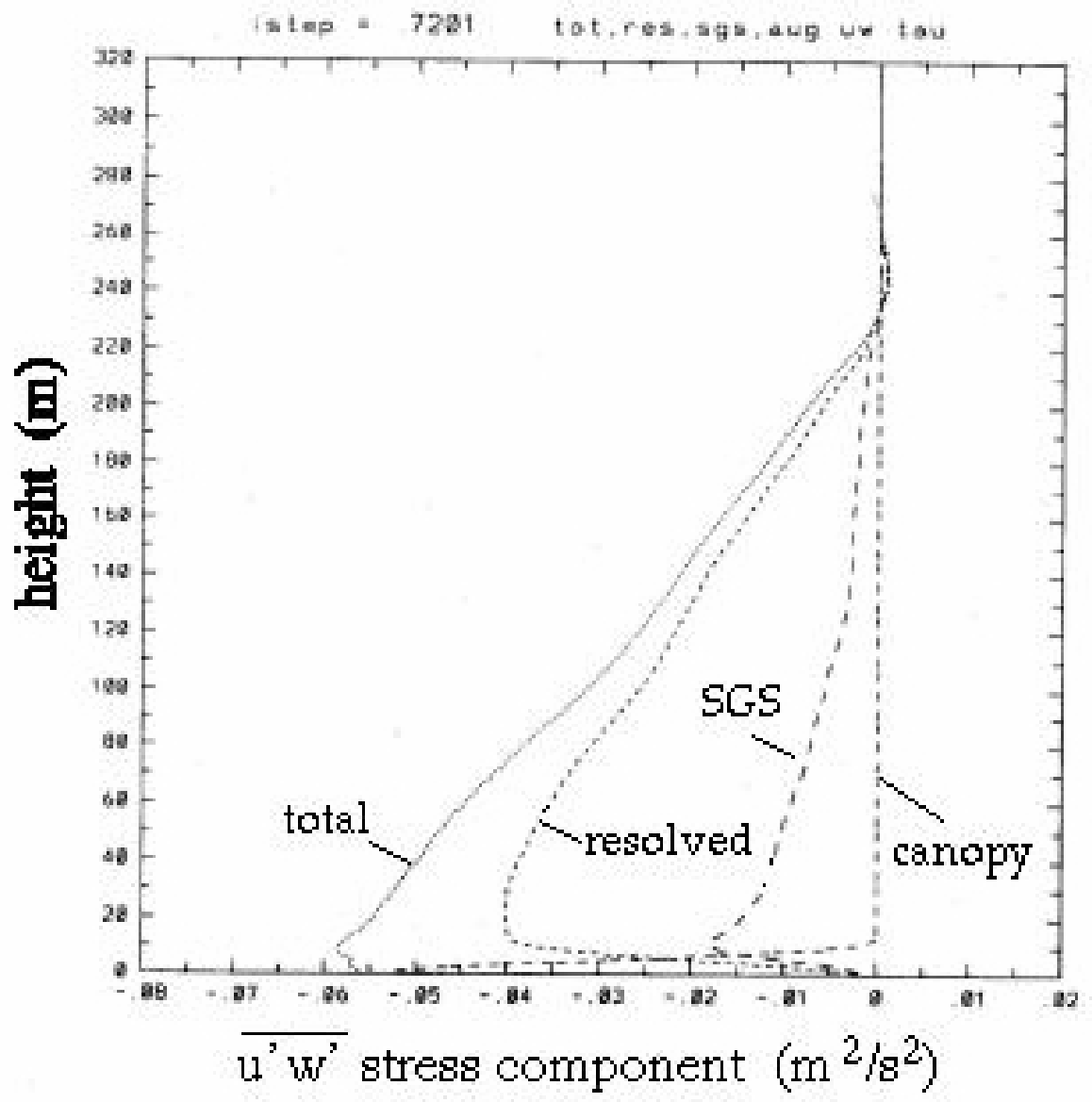

(b)

Figure 5.5: Augmentation of stress profiles using the 'canopy' approach of Brown (2001); the 'notch is evident before (a), but is filled after (b); the SGS, resolved, and canopy stress components and the total stress are shown. 


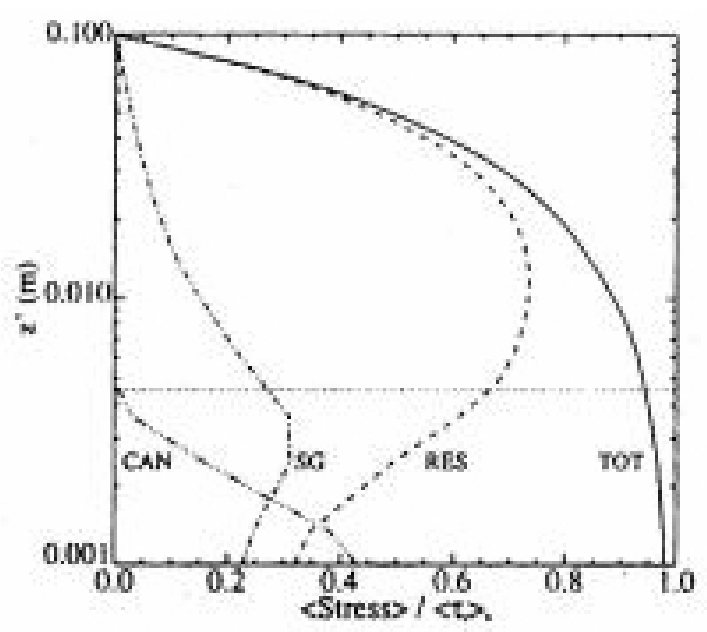

(a)

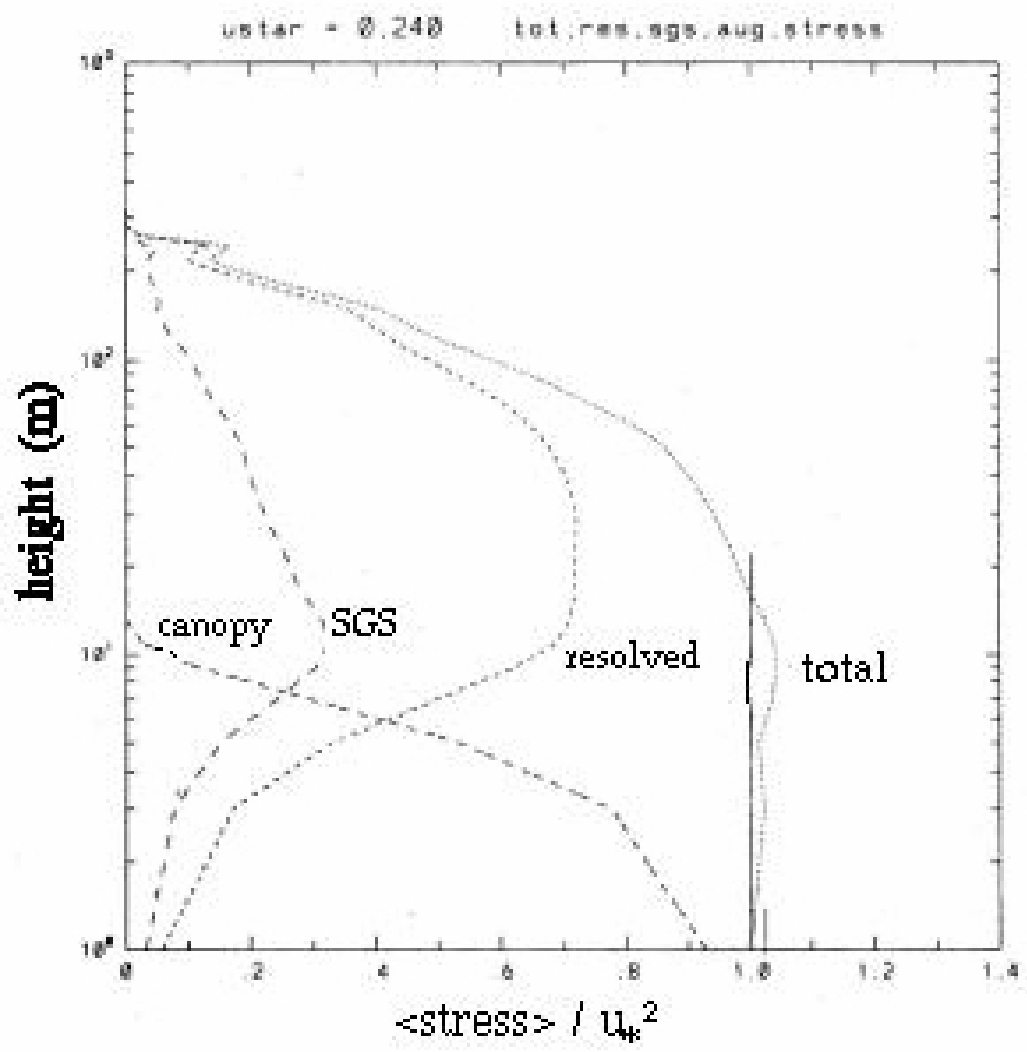

(b)

Figure 5.6: Comparison of stress profiles using the 'canopy' approach of (a) Brown (2001) versus (b) as implemented in this LES model; the SGS, resolved, and canopy stress components and the total stress are shown; the vertical line indicates $\langle$ stress $\rangle=u_{*}^{2}$. 


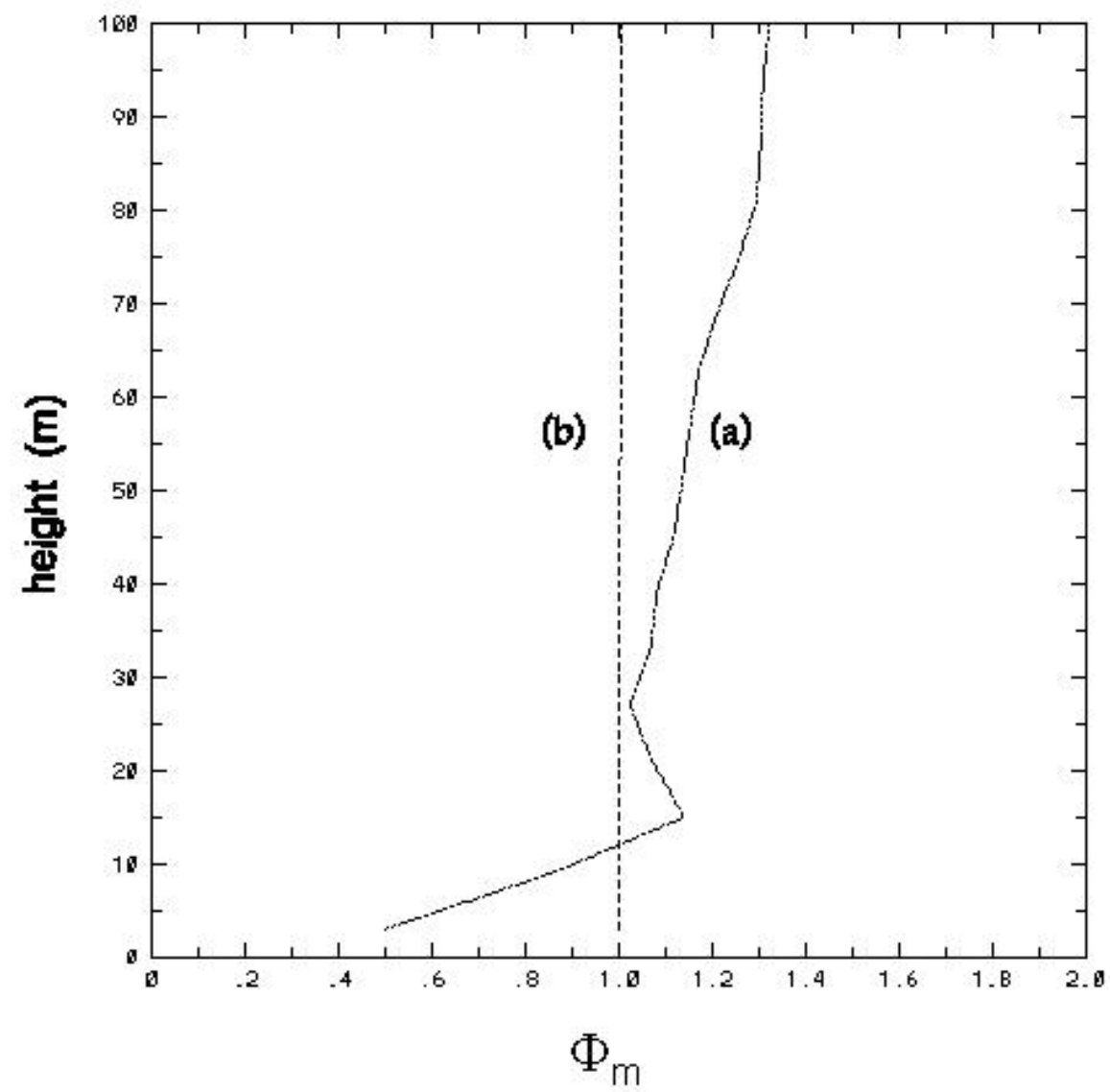

Figure 5.7: Estimate of nondimensional wind shear $\phi_{m}$ for the neutral portion of Run G7.5wt-02; solid line (a) is from LES results, while dashed line (b) is from Dyer relation $\phi_{m}=1+5 z / L$. 


\section{Chapter 6}

\section{Features of the Evolving SBL}

\subsection{Forcing the Simulated SBL}

The evolution of the SBL is governed primarily by two external forcings: the geostrophic wind and the surface cooling. The relative strengths of these two forcings will determine the eventual structure of mean and turbulence properties of the SBL. In particular, the stronger the geostrophic wind, the greater the mixing, the deeper the SBL, and the weaker the surface-based temperature inversion. On the other hand, the stronger the cooling, the stronger the surface-based inversion.

The parameter space of these two forcings is explored in this research. The simulations, summarized in Table 6.1, cover a range of geostrophic winds above a below a reference value of $7.5 \mathrm{~m} / \mathrm{s}$. Rates used for the surface cooling are within ranges observed in the field. Note that the run names in Table 6.1 capture the forcings used, where ' $G$ ' precedes the geostrophic wind speed $(\mathrm{m} / \mathrm{s})$ and 'wt' precedes the surface cooling $(\mathrm{K} \mathrm{m} / \mathrm{s})$. A suffix is used to indicate modifications, most often for the grid used. The geostrophic wind is applied such that the near surface flow is approximately west to east, aligned with the x-axis, with Run G5wt-02N being an exception. Generally the geostrophic wind is rotated about 30 degrees clockwise from the surface wind. The latitude is 43.44 degrees $\mathrm{N}$, which gives a value for the Coriolis parameter of $10^{-4}$. The afternoon, evening transition, and nighttime periods are simulated in Run G10.4wt-02T. In all other runs, a neutral boundary layer is simulated before the onset of cooling; this would be typical of a cloudy day, where little surface heating occurs.

Mason and Derbyshire (1990) studied this parameter space and identified a maximum surface cooling, beyond which turbulence would be extinguished. They related the surface cooling to the downward surface buoyancy flux $\left(B_{0}\right)$, with buoyancy $(b)$ 
related to potential temperature by $b=g\left(\theta-\theta_{0}\right) / \theta_{0}$ where $\theta_{0}$ is the reference temperature for the Bousinesq approximation. The parameter $B_{0}$ is related to the lower boundary condition for potential temperature, which is usually given by a prescribed surface heat flux or cooling rate. Since the forcing used here is in terms of surface heat flux, the following definition is used

$$
B_{0}=-\left(g / \theta_{0}\right)\left(\overline{w^{\prime} \theta^{\prime}}\right)_{0}
$$

If the downward surface buoyancy flux is too large (i.e. $B_{0}>B_{\max }$ ), then turbulence cannot be maintained. Derbyshire extended the steady-state SBL model equations of Nieuwstadt (1985) to define $B_{\max }$. Using the definition for the MoninObukhov length L

$$
L=\frac{-u_{*}^{3}}{k(g / \theta) \overline{w^{\prime} \theta^{\prime}}},
$$

and the definition of $u_{*}$ consistent with Nieuwstadt's model

$$
u_{*}=G k R_{f} L / h
$$

one can restate the surface buoyancy flux as

$$
B_{0}=\frac{u_{*}^{3}}{k L}=G^{2} k^{2} R_{f}^{2} \frac{L^{2}}{h^{2}} \frac{u_{*}}{k L}
$$

Using the definition of SBL depth $\mathrm{h}$

$$
h^{2}=\sqrt{3} k R_{f} u_{*} L /|f|
$$

and a critical value of the flux Richardson number $R_{f c}$, Derbyshire expressed the stable limit of the surface buoyancy $B_{\max }$ as

$$
B_{\max }=\frac{R_{f c}}{\sqrt{3}} G^{2}|f|
$$

With a critical Richardson number $R_{f c}=0.25$, a geostrophic wind $\mathrm{G}=7.5 \mathrm{~m} / \mathrm{s}$, and a Coriolis parameter $f=10^{-4} s^{-1}$, one obtains $B_{\max }=8.12 \times 10^{-4} \mathrm{~m}^{2} \mathrm{~s}^{-3}$; note that the value is positive because it is defined as downward buoyancy flux. It is 
slightly larger than the value $B_{0}=6.76 \times 10^{-4} \mathrm{~m}^{2} \mathrm{~s}^{-3}$ obtained for the moderately stable run (G7.5wt-02), using a reference temperature $\theta_{0}=290 \mathrm{~K}$ in Equation 6.1. For the more stable reference run $\left(G^{7} .5 w \mathrm{t}\right.$-02) with $G=5 \mathrm{~m} / \mathrm{s}$, the limiting value obtained is $B_{\max }=3.61 \times 10^{-4} \mathrm{~m}^{2} \mathrm{~s}^{-3}$. In this case, the downward buoyancy flux for the surface cooling used is $B_{0}=6.76 \times 10^{-4} \mathrm{~m}^{2} \mathrm{~s}^{-3}$, which exceeds $B_{\text {max }}$, and yet turbulence remains (though strongly damped). Derbyshire (1990) offered a more robust (and less stringent) value for $B_{\max }$ based on a gross energy balance for the quasi-steady SBL

$$
B_{\max }=4 R_{f c} G^{2}|f|
$$

The limiting value of $B_{\max }$ in Equation 6.7 is about 7 times greater than that in Equation 6.6, and exceeds the value of $B_{0}$ for Run $G 5 w t$-02 by a factor of 3.7. However, Derbyshire views Equation 6.7 as a generous upper bound, while the lower value (Equation 6.6) is more correct, as verified in his study.

An alternative is to use a larger value for $R_{f c}$. Although the critical Richardson number for transition from laminar to turbulent flow may be 0.25 , the critical value for transition from turbulent back to laminar flow may well be much larger, such as a value of 1 (Stull, 1988). Using a value of $R_{f c}=1$ gives a stable limit $B_{\max }$ that is 4 times greater than that with $R_{f c}=0.25$. For Run G5wt-02, $B_{\max }=14.4 \times 10^{-4} \mathrm{~m}^{2} \mathrm{~s}^{-3}$, which exceeds $B_{0}$ by a factor of 2.1. This is a more satisfactory result since the turbulence is not extinguished in that run. For Run ( $\left.{ }^{7} 7.5 w t-02\right)$, the revised value of $B_{\max }$ is $32.5 \times 10^{-4} \mathrm{~m}^{2} \mathrm{~s}^{-3}$, which exceeds $B_{0}$ by a factor of 4.8 .

By setting $B_{0}$ equal to $B_{\max }$, one can develop a criterion for determining whether the turbulence in the SBL is continuous or intermittent. Using the definition of $B_{0}$ and $B_{\text {max }}$ in Equations 6.1 and 6.6, respectively, one has $-\left(g / \theta_{0}\right)\left(\overline{w^{\prime} \theta^{\prime}}\right)_{0}=(1 / \sqrt{3}) R_{f c} G^{2}|f|$. Put in another way, for a given geostrophic forcing, the maximum amount of surface cooling that can be present and still allow continuous turbulence using the Derbyshire criterion with $R_{f c}=1$ is

$$
\left(\overline{w^{\prime} \theta^{\prime}}\right)_{0_{\text {maximum cooling }}}=-17.1 \times 10^{-4}\left(K s m^{-1}\right) G^{2}
$$

given $f=10^{-4} \mathrm{~s}^{-1}, g=9.8 \mathrm{~m} \mathrm{~s}^{-2}$, and $\theta_{0}=290 \mathrm{~K}$. 
The pairs of forcings used in this research are summarized in Figure 6.1, along with the criterion curve defined by Equation 6.8 which uses $R_{f c}=1$, and also a curve for reference that is based on $R_{f c}=0.25$ which results in a leading coefficient that is a fourth of the value in Equation 6.8. Combinations of geostrophic forcing and surface cooling can be collected in three zones where (1) continuous turbulence and enhanced periods of turbulence occur routinely (the upper right), (2) the turbulence continues but the occurrence of enhanced turbulence periods is reduced (middle), and (3) where the turbulence is not expected to be maintained (lower left). As will be shown in this chapter, use of surface cooling that exceeds the criterion with $R_{f c}=0.25$ leads to results with fewer enhanced turbulence periods. However, this criterion is overly strict, and simulations with such values of surface cooling still exhibit continuous turbulence. The groups of scenarios with the $-0.02 \mathrm{~K} \mathrm{~m} / \mathrm{s}$ and $-0.04 \mathrm{~K} \mathrm{~m} / \mathrm{s}$ surface cooling crosses through all three zones. As the Derbyshire criterion with $R_{f c}=1$ is approached, the amount of turbulence is dramatically reduced.

In the sections that follow, the evolution of the simulated SBL is described. Since periodic lateral boundary conditions are used in these simulations, the evolution of the boundary layer must be described in terms of the temporal change in horizontallyaveraged statistics. This is the analog to the downstream evolution documented in laboratory studies described in Section 2.2. If the simulations used inflow boundary conditions, then a more direct comparison with laboratory studies would be possible.

\subsection{Mean Atmospheric Properties}

Comparisons between CBL and SBL characteristics are done with results from Run G10.4wt-02T. In this run, the prescribed surface heat flux for the CBL is $0.06 \mathrm{~K} \mathrm{~m} / \mathrm{s}$ $\left(75 \mathrm{~W} / \mathrm{m}^{2}\right)$. The CBL is established before the onset of surface cooling. The surface heat flux is then decreased linearly over a 1 hour period to $-0.02 \mathrm{~K} \mathrm{~m} / \mathrm{s}\left(-25 \mathrm{~W} / \mathrm{m}^{2}\right)$ to represent the period around sunset. The surface flux then remains constant during the rest of the simulation as the SBL develops. The mean properties of the simulated CBL and SBL agree well with those observed, as summarized in Subsection 2.1.1. As seen in Figure 6.2, the CBL has a well-mixed layer characterized by uniform vertical profiles of mean temperature and wind speed. In contrast, the SBL has very strong 
vertical gradients of temperature and wind speed. The SBL also has a local maximum in the wind speed (the low level jet) near the top of the surface-based inversion.

Vertical profiles for mean temperature and wind speed are shown for the two references cases in Figures 6.3 and 6.4. For both cases, the surface cooling is the same. The characteristics of the resulting SBLs are obtained from vertical profiles of potential temperature and wind speed averaged over 1 hour between hour 8 and hour 9 of the simulations, and include the SBL depth, inversion strength, and wind speed maximum of the low level jet. The inversion strength is the mean potential temperature gradient from the surface to the top of the SBL expressed in degrees $\mathrm{K}$ per $100 \mathrm{~m}$. For the higher wind speed stable case, the resulting SBL is deeper (170 $\mathrm{m})$ and the strength of the surface-based inversion is weaker $(3.1 \mathrm{~K} / 100 \mathrm{~m})$ than for the the lower wind speed case, where a more shallow $(70 \mathrm{~m})$ and more stable $(18.0$ $\mathrm{K} / 100 \mathrm{~m})$ SBL develops. After 7 hours of constant cooling, the higher wind speed case (G7.5wt-02) has developed a low level jet, which, with a wind speed maximum of $9.8 \mathrm{~m} / \mathrm{s}$, is a $31 \%$ increase over the geostrophic forcing. The jet for the lower wind speed case (G5wt-02) has a maximum of $7.1 \mathrm{~m} / \mathrm{s}$, which is a $42 \%$ increase over the geostrophic forcing.

The evolution of the surface-based temperature inversion, which is a good surrogate for the SBL location is shown in Figure 6.5 for the moderately stable case in Run G7.5wt-02, and in Figure 6.6 for the more strongly stratified case in Run G5wt-02. The point where the tight vertical packing of the contours begins to relax is a good indication of the SBL top. Visually, the demarcation between the dark and light green gives an idea of how the SBL evolves, but it not represent the entire depth of the SBL. Over time, the increased mixing associated with higher levels of mechanical turbulence from higher wind speeds is evident in the growth of the deeper SBL in Run G7.5wt-02. In contrast, for the lower wind speeds in Run G5wt-02, the evolution of a more shallow SBL.

The characteristics of several SBLs that evolved over 9 hours of simulation are summarized in Table 6.2. In general, over the range of forcings used, the resulting SBLs show the following tendency: higher geostrophic forcing for a given surface cooling leads to weaker, deeper surface-based temperature inversions due to mixing 
and hence less stable SBLs, while stronger surface cooling at a given geostrophic forcing leads to stronger, more shallow surface-based temperature inversions and hence more stable SBLs. The forcing terms can be expressed as quantities with common units, namely buoyancy flux $B_{0}$ (see Equation 6.1) and an inertial term $G^{2}|f|$. The pairs of forcings can be combined in a "stability forcing index" $F_{s}=B_{0} / G^{2}|f|$; Derbyshire (1990) identified this ratio as a useful predictive measure of atmospheric stability in the resulting SBL. This index is included in Table 6.2.

To quantify the relationship between the forcing and the resulting stability of the SBL, the values that characterize the SBL (depth $h$, inversion strength $\Delta \theta / h$, and low level jet $u_{j e t}$, as summarized in Table 6.2) are used to form a bulk Richardson number

$$
R_{B}=\frac{\left(g / \theta_{0}\right) \Delta \theta h}{u_{\text {jet }}^{2}} .
$$

The relationship between forcing and resulting bulk stability is very strong indeed, as seen in Figure 6.7, and can be expressed as

$$
R_{B}=\frac{8}{3} F_{s}
$$

The strong correlation between the two terms in Equation 6.10 is not surprising. The denominators of both terms are versions of the square of the flow speed $(G$ and $\left.u_{j e t}\right)$ and thus are highly correlated. The numerators are related, but not as directly as the denominators. The numerator in $F_{s}$ represents the surface buoyancy flux (a sink of thermal energy). Over time, the loss of thermal energy will be reflected in a temperature deficit $\Delta \theta$ over some depth $h$. The numerator of $R_{B}$ captures this loss of thermal energy as $\Delta \theta h$, so indeed the numerators are expected to be correlated as well. Nonetheless, this is a valuable finding. The simple relationship in Equation 6.10 is a powerful predictive tool for practical applications. The bulk stability of the evolving SBL can be predicted if the geostrophic and buoyancy forcing are known. From routine measurements, one can obtain estimates of the forcing, and hence an estimate of the bulk stability of the SBL (in the absence of direct measurements from a tall tower or remote sensing instruments). Indeed, such estimates can be obtained in a forecast mode using output from numerical weather prediction models and knowledge of the local surface conditions. In this way, forecasts of the bulk stability of the SBL 
can be made.

The forcing parameter space illustrated in Figure 6.1 can be recast in terms of the forcing in common units, namely buoyancy flux and $G^{2}|f|$. The forcing pairs for the simulations are plotted in Figure 6.8, along with the Derbyshire criterion using $R_{f c}=1$ and $R_{f c}=0.25$. The forcing pairs are plotted with three different symbols, depending on whether the value of $R_{B}$ from Equation 6.10 is in the range $0<R_{B} \leq 0.25,0.25<R_{B} \leq 1$, or $R_{B}>1$. The three points that fall clearly to the left of the criterion are those simulations where $B_{0}>B_{\max }$. These are the same simulations where points are substantially above $R_{B}=1$ in Figure 6.7, and are simulations where turbulence is not expected to be continuous. See more discussion of two of these simulations in Subsection 6.3.1 and Figures 6.14, 6.15, and 6.18. The values of $R_{B}$ from Equation 6.10 are in reasonable agreement with the Derbyshire criterion, which demonstrates the consistency between the predictive relationship and the Nieuwstadt SBL model.

In Subsection 2.1.1, a relation is discussed between the wind speed and surface cooling and the resulting SBL depth $\left(h_{s}\right)$. From data collected during an experiment in Minnesota (as seen in Figure 2.2), the relation is quantified in terms the friction velocity, $u_{*}$, and negative surface heat flux $H$ (in $W / m^{2}$ )

$$
h_{s}=\frac{21500 u_{*}^{2}}{\sqrt{H}}
$$

Values for friction velocity have been obtained from the total surface stress for the runs summarized in Table 6.2. These values and the surface cooling are used to calculate SBL depths from Equation 6.11, which are then compared with the LES-derived SBL depths. As shown in Figure 6.9, the agreement is good in the mid-range of SBL depths. However, at the extremes, the agreement is not as good. The Minnesota algorithm tends to overpredict when SBL values are large, and underpredict when they are small, relative to the LES-derived SBL depths. 


\subsection{Turbulence Properties}

\subsubsection{Velocity variances}

The velocity variance for each component is computed at each time step for each vertical level from squared deviations from the horizontal average value. For example, for the w-component,

$$
\sigma_{w}^{2}=\left(1 / N_{k}\right) \sum_{k=1}^{N_{k}}\left(w_{k}-\langle w\rangle\right)
$$

where $N_{k}=N_{x} N_{y}$ is the number of horizontal grid points. The variances are then averaged in time over 5 minutes.

Vertical profiles of velocity variance for each velocity component are compared in Figure 6.10 for the CBL and SBL simulated in Run G10.5wt-02T. Note that the horizontal scales are the same between CBL and SBL plots, which emphasizes the drastic reduction in variances in the SBL compared to the CBL. The vertical velocity variance is of comparable size to the other velocity variances through much of the CBL, and this contributes to the strong vertical mixing associated with large, convectivelydriven eddies. In the SBL, the vertical velocity variance is a small fraction of the other velocity variances; this anisotropy is due to the preferential suppression of vertical fluctuations by buoyancy in stably stratified conditions.

The time behavior of the reduction of turbulence through the boundary layer is evident in Figure 6.11, which shows the time history of the vertically-integrated velocity variances by component as the CBL to SBL transition occurs. The preferential reduction of turbulence in the vertical velocity component is clearly illustrated as the SBL develops. The variability of turbulence in the SBL is evident, with a period of enhanced turbulence occurring at hour 10.

Spectra of velocity variances are shown in Figure 6.12 for each velocity component before, during, and after 'enhanced' turbulence event at hour 10. During the event, the energy is increased fairly evenly across all scales compared to that before the

event. In the relaxation period after the event the decrease in energy is greater in the small scales compared to the small scales, and the spectral peak moves to larger scale (lower wavenumber). The damping of the enhanced turbulence in the small scales after the event is especially noticeable in the $\mathrm{v}$ and $\mathrm{w}$ velocity variances. 
Time histories of velocity variance for 3 other scenarios of geostrophic forcing and cooling are shown in Figure 6.13. The time history for the same scenario (G10.4wt02T) shown in Figure 6.12a for reference. As the surface cooling is doubled (Run G10.4wt-04T) in Figure 6.12b, the turbulence decreases just slightly but there is still an enhanced turbulence event at hour 9. However, if the surface cooling is held constant and the geostrophic forcing is reduced by a factor of two (as seen in Figure 6.12c for Run G5.2wt-02T), the reduction in turbulence is more dramatic, with little enhance turbulence. Reduction of the geostrophic wind by another factor of two (see Figure 6.12d for Run G2.6wt-02T) leads to a little more reduction in turbulence during the initial transition, but is comparable to that in Run G5.2wt-02T later in the period. This comparison is consistent with the criteria presented in Equations 6.6 and 6.7, where the effect of geostrophic wind is squared compared to the surface cooling. That is, the amount of mechanical energy available from the geostrophic forcing is the dominant forcing, with a lesser modification by the surface cooling.

Time histories of velocity variance for the two reference runs $(G \% .5 w t-02$ and G5wt-02) are shown in Figures 6.14 and 6.15. In Run G\%.5wt-02, the turbulence remains fairly active with a broad period of enhanced turbulence around hour 7 , and a sharp event just after hour 11 . This second event will be investigated further in Section 6.4. The impact of this event can be seen in the evolving temperature profile in Figure 6.5, where there is a slight ripple just after hour 11. In the more stable reference run (G5wt-02), there is much less turbulence overall and much less enhanced turbulence activity.

Two simulations (G2.5wt-04M and G1.5wt-02M) were performed in the zone where continuous turbulence is not expected to be maintained. Time histories of velocity variance for these two simulations (see Figures 6.16 and 6.17) show that turbulence is reduced but is still maintained; note that the vertical scale in these figures is $1 / 4$ that in Figures 6.14 and 6.15 to assist visualization. What has changed in this zone is that the vertical velocity fluctuations are more comparable to those of the horizontal velocity. In this zone, the vertical velocity variance may be representing variations that are not strictly turbulence, but rather include wave-like activity. It is more correct to say that there are periods of enhanced variability, rather than enhanced turbulence. 
Indeed the vertical velocity variance is the largest of the three components in the enhanced variability period at hour 6 in the higher wind speed run (G2.5wt-04M) and in all three enhanced variability periods in the lower wind speed run (G1.5wt-02M). In Figure 6.17 for the low wind speed case, the onset of the enhanced variability is seen first in the vertical component, while the horizontal components lag. This case has the lowest geostrophic forcing of all the simulations, and thus the effects of vertical perturbations have relatively more weight. The vertical profile of the $u$ velocity variance at hour 7 has its maximum at a height of $47.5 \mathrm{~m}$ (with values nearly that large at $42.5 \mathrm{~m}$ ) and then decreases smoothly above that height. The welocity variance profile has a minimum at a height of $40 \mathrm{~m}$ and has a broadly increasing value with a smooth peak at about 90-m height. The top of the strong, surface-based temperature inversion (and hence the SBL) is at about the 40-m height. Spectra of the $\mathrm{u}$ and $\mathrm{w}$ components of velocity at the upper height $(92.5 \mathrm{~m}$ for $\mathrm{u}$ and $90 \mathrm{~m}$ for $\mathrm{w}$ due to the staggered grid) show that the $\mathrm{w}$ component carries more energy than the $\mathrm{u}$ component (see Figure 6.18a); this case is reversed at the lower height height (see Figure 6.18b) where the u component carries much more energy than the w component. The enhanced activity at the upper level may well be caused by an increase in wave activity above the inversion.

A more complete way to view the evolution of the turbulence in the evolving SBL is through a time-height cross section. This done for Run G7.5wt-02 is in Figure 6.19 for the 14 hours of the simulation. The temporal behavior of the TKE is consistent with the time history in Figure 6.14. The sharp event just after hour 11 has its peak TKE in the upper portion of the SBL; this suggests that the event is not related to shear generated turbulence near the surface. The rather uneventful evolution of the more stable reference case (G5wt-02) is evident in Figure 6.20.

The shear stress is reduced in response to the surface cooling, as is the friction velocity. The evolution of $u_{*}$ is investigated for simulations where the geostrophic wind is constant and the surface cooling is varied; this is the case for Runs G5wt01, G5wt-02, and G5wt-04. The results are similar to those reported by Wyngaard (1975) and shown in Figure 4.2b. The relationship of surface cooling values to each other is like the lowest 3 curves in the figure, but the starting $u_{*}$ value in the current simulations for $G=5 \mathrm{~m} / \mathrm{s}$ is lower (0.25). For the current simulations, the drop to a 
minimum value is faster for the stronger surface cooling than for the weaker surface cooling; the drop is $1.6 \mathrm{~h}$ for Run G5wt-04, $1.8 \mathrm{~h}$ for Run G5wt-02, and $2.0 \mathrm{~h}$ for Run G5wt-01. The amount of decrease from the starting value of $0.25 \mathrm{~m} / \mathrm{s}$ is to $0.9 \mathrm{~m} / \mathrm{s}$ for Run G5wt-04, $1.1 \mathrm{~m} / \mathrm{s}$ for Run G5wt-02, and $1.2 \mathrm{~m} / \mathrm{s}$ for Run G5wt-01.

\subsubsection{Temperature variances}

The temperature variances are calculated with the approach in Equation 6.12 using the potential temperature field at each model level. The evolution of temperature variance for Run G7.5wt-02 are shown in Figure 6.21, where the temperature variance is relatively small until the event after hour 11. Again the maximum is in the upper part of the SBL. The evolution of temperature variance for Run G5wt-02 is shown in Figure 6.22. Here the variance is larger over more of the simulation than in the less stable reference case. The maximum values are also in the upper part of the SBL throughout the simulation.

\subsection{An Episode of Enhanced Turbulence}

There are several episodes of enhanced turbulence in the SBL simulations. Two are analyzed in this research. The first is highlighted in Figure 6.11 for Run G10.4wt-02T. This event is used in the dispersion study summarized in Chapter 8.

A second episode of enhanced turbulence is the rather sharp event occurring after hour 11 in Run G\%.5wt-02, and highlighted in Figures 6.14. Here the episode is described in some detail, sort of an anatomy of an episode. An expanded time history of the 5-minute velocity variances for the 30 minutes after hour 11 is shown in Figure 6.23. The onset of the event occurs at 15 minutes after hour 11 (or hour 11.25). Several figures follow which compare 5-minute averaged variables at the onset time and 15 minutes later (30 minutes after hour 11 or hour 11.5).

The vertical profiles of mean potential temperature and wind speed change very little during the 30 minutes; thus only the profiles at hour 11.5 are shown for reference in Figure 6.24. The top of the SBL is at about $190 \mathrm{~m}$, although there is a twolayer structure where the bottom layer below about 100-m height has a stronger 
temperature gradient (more stable) with greater wind shear. The two-layer structure of the SBL is discussed further in Section 7.6.

\subsubsection{Change in Vertical SBL Structure}

From the onset of the episode (hour 11.25 to the peak of the episode hour 11.5) there is a strong increase in the velocity variances, as illustrated in Figures 6.25 and 6.26, as is also the case for the potential temperature variance (see Figure 6.26). Interestingly, at the peak of the episode, the maximum in the $u$ velocity variance is near the top of the lower SBL layer, while that for the v velocity variance is in the upper SBL layer. The maxima of the $\mathrm{w}$ velocity variance and potential temperature variance are near the top of the lower SBL layer.

The stresses and heat flux are consistent with time change in variances. At the episode peak, the $\overline{u^{\prime} w^{\prime}}$ stress has a secondary maximum near the top of the lower SBL layer, while the $\overline{v^{\prime} w^{\prime}}$ has its primary maximum just above that in the upper SBL layer, as shown in Figure 6.27. The heat flux, shown in Figure 6.28, has its maximum during the episode peak at the top of the SBL lower layer; interestingly, at the onset of the episode, there is a secondary maximum in the upper SBL layer.

The SGS turbulence model responds to the change in conditions between the onset and peak of the episode. The SGS dynamic coefficients show a strong increase near the base of the upper SBL layer (see Figure 6.29). The transfer of SGS TKE, illustrated in Figure 6.30, shows a strong increase in the forward scatter (downscale transfer) throughout the SBL during the episode, with a maximum near the top of the lower SBL layer. The backscatter (upscale transfer) of SGS TKE has a relatively small increase in the upper half of the lower SBL layer and through the upper SBL layer. The response of the SGS thermal energy is quite different. The backscatter component shows an increase more comparable to the increase in the forward scatter component (see Figure 6.31). The maximum too is near the top of the lower SBL layer. During the simulated episode, both the forward scatter and backscatter of SGS thermal energy increased, and the net SGS thermal energy transfer downscale also increased, especially in the region of the episodic activity (in the mid to upper SBL). The increase in net SGS TKE and thermal energy transfer during the episode is 
consistent with the observations made by Coulter (1990) of turbulent episodes in the SBL (see Figure 2.18) where increases in dissipation rates of TKE and temperature variance occurred with increases in the vertical velocity RMS.

\subsubsection{Wave-Like Undulations during the Episode}

Temperature contours in the $\mathrm{x}-\mathrm{z}$ plane are very illustrative of variability in the SBL. They are used here, with overlays of fluctuating velocity vectors, to highlight the dynamics of the turbulence episode. Instantaneous values of temperature and velocity are used in these plots; no spatial or temporal averaging has been applied. Note that the vertical scale is stretched by factor of two, and the fluctuating velocities scaled by factor of 20 to assist in visualizing the flow perturbations. The $\mathrm{x}-\mathrm{z}$ plane is located at $\mathrm{y}=900 \mathrm{~m}$. For reference, the temperature contours at the onset of the epsisode (60 sec after hour 11.25) are shown in Figure 6.32. The SBL is smoothly stratified with a distinct temperature gradient in the vertical direction and very little horizontal variation in the temperature field.

In the next 8 figures, covering a period from 480 to 522 seconds after hour 11.25, a wave-like pattern with apparent overturning is captured. In Figure 6.33, a deep yellow intrusion of warm air is seen in the center of the figure. Six seconds later in Figure 6.34, a push of cooler air in a wave-like form begins to overtake this region as the pattern is transported left to right at about $9 \mathrm{~m} / \mathrm{s}$ in the mean wind. At this point, temperature in the core of the yellow area (now more red) is clearly warmer then the air above it, indicating a region of thermal instability. Dörnbrack (1998) has pointed out the role of thermal instability in the early stages of turbulent mixing by gravity waves. However, the dynamic effects are likely dominant; for example, a well-defined eddy motion is just up and to the right of the pattern of interest. Over the next 12 seconds, the cooler air in the overtaking (and appearing to be overturning) motion from the left has created a closed off pocket of relatively warm air (see Figure 6.36). Over the following 24 seconds, the warm pocket is mixed into the cooler air (see Figures 6.37 - 6.40). The relative importance of various physical mechanisms in the mixing process here is not clear; however, it would appear that the dynamics in the upper layer of this SBL create an overturning, and that the thermal 
instability supports the small scale mixing. The grid resolution in this simulation may be too coarse to capture adequately the small scale processes. Results from a simulation with finer resolution $(G 5 w t-02 F)$ is used to investigate details of the flow further in Section 7.6.

Near the peak of the turbulence episode, 570 seconds after hour 11.25, a large structure is evident in the right-center part of the Figure 6.41. The fluctuating velocity field has a large downward thrust supporting the intrusion of warmer air. To help visualize the larger picture, an isosurface of potential temperature $(286.5 \mathrm{~K})$ is shown in Figure 6.42. The vertical axis is height $(0-240 \mathrm{~m})$ and the horizontal axes are $(0-960 \mathrm{~m})$ with the $\mathrm{x}$-axis (west-east) from left to right along the grid lines and the y-axis (south-north) from bottom to top along the grid lines; the vertical scale is stretched by factor of two for visualization. The $\mathrm{x}-\mathrm{z}$ plane in the previous figures is near the top of the figure, and is oriented vertically from left to right. The isosurface value was chosen to represent the boundary between the darker and lighter green in Figure 6.41. As can be seen in Figure 6.42, there are wave-like undulations in the temperature field across the entire grid. This confirms that what is seen in the $\mathrm{x}-\mathrm{z}$ plane at $\mathrm{y}=900 \mathrm{~m}$ is likely to be seen at other $\mathrm{y}$-axis locations.

Temperature contours in the $y-z$ plane help illustrate spanwise structure in the SBL during the episode. The combination of contours in the $\mathrm{x}-\mathrm{z}$ plane and $\mathrm{y}-\mathrm{z}$ plane gives a more complete picture than just one plane alone. To complement the analyses previous shown for the $\mathrm{x}-\mathrm{z}$ plane, selected periods will be shown in the $\mathrm{y}-\mathrm{z}$ plane at $\mathrm{x}$ $=670 \mathrm{~m}$, the location of a significant overturn seen in the $\mathrm{x}-\mathrm{z}$ plane analyses at about 492 seconds into the episode (see Figure 6.43). Fluctuating velocity components in the $\mathrm{y}-\mathrm{z}$ plane are included in the plots that follow. Near the onset of the episode, there is a large scale undulation midway up in the SBL, as shown in Figure 6.44. The undulation become very pronounced about 2 minutes later (see Figure 6.45). About 5 minutes later, small scale disturbances are dominating the upper part of the SBL, and the large-scale undulations are broken up into smaller scales, as seen in Figure 6.46. On the right side of the figure midway up into the SBL, there is a well-defined circulation at about the point where overturning was seen in the $\mathrm{X}-\mathrm{Z}$ plane analysis (see Figure 6.35). Approaching the episode peak, the SBL becomes even more disturbued, with stronger vertical exchange (see Figure 6.47). Even the 
surface layer is perturbed, with warmer air being swept down near the ground in the region near $\mathrm{y}=500 \mathrm{~m}$ to $\mathrm{y}=700 \mathrm{~m}$. This exchange was not evident in the $\mathrm{x}-\mathrm{z}$ analysis since the $\mathrm{x}-\mathrm{z}$ plane was at $\mathrm{y}=900 \mathrm{~m}$.

The large undulations noted in Figure 6.45 are seen dramatically in the $288 \mathrm{~K}$ isosurface of potential temperature in Figure 6.48 at 3 minutes into the episode. The effect of the smaller scale turbulent motions in breaking up this large-scale feature is evident in Figure 6.49, although there is some remnant of the large-scale organization.

The flow at the height of this isosurface is about aligned with the x-axis. What is seen in the $\mathrm{x}-\mathrm{z}$ plane analysis is aligned with the mean flow ( \pm 10 degrees) in the height range of about $50-100 \mathrm{~m}$ (the top half of the lower SBL layer). Note that in the upper layer of the SBL, the wind direction continues to rotate another 24 degrees clockwise, overshooting the direction of the geostrophic forcing by 7 degrees. This directional shear is likely the cause of the large $\mathrm{v}$ velocity variance and $\overline{v^{\prime} w^{\prime}}$ stress in the upper SBL layer.

The episode of enhanced turbulence described has similar features to that documented on the Cabauw tower (see Figure 2.9). There the enhanced activity was in the upper part of the SBL. The time period of the episode was about an hour, with a similar ramping up period of 10-15 minutes. The episodic behavior of turbulence in the SBL observed by Coulter (1990) is also similar to the event described here (see Figure 2.18). 
Table 6.1: Summary of Simulations of SBL.

\begin{tabular}{|c|c|c|c|c|c|}
\hline Name† & $\begin{array}{c}\text { Geostrophic } \\
\text { Forcing } \\
(\mathrm{m} / \mathrm{s})\end{array}$ & $\begin{array}{l}\text { Surface } \\
\text { Cooling } \\
(K \mathrm{~m} / \mathrm{s})\end{array}$ & $\begin{array}{l}\text { Domain } \\
L_{x} L_{y} L_{z} \\
\quad(m)\end{array}$ & $\begin{array}{l}\text { Grid Res. } \\
\Delta x, y, z \\
(m)\end{array}$ & $\begin{array}{l}N \text { of cells } \\
N_{x} N_{y} N_{z}\end{array}$ \\
\hline G7.5wt-02 & 7.5 & -0.02 & 9096960480 & 1515156 & 646480 \\
\hline G5wt-02 & 5.0 & -0.02 & 960960480 & 15156 & 646480 \\
\hline G10wt-02 & 10.0 & -0.02 & 960960480 & 15156 & 646480 \\
\hline G5wt-01 & 5.0 & -0.01 & 960960480 & 15156 & 646480 \\
\hline G7.5wt-02F & 7.5 & -0.02 & 320320400 & $\begin{array}{lll}5 & 5 & 5\end{array}$ & 646480 \\
\hline G5wt-02F & 5.0 & -0.02 & 320320400 & $\begin{array}{lll}5 & 5 & 5\end{array}$ & 646480 \\
\hline G5wt-01F & 5.0 & -0.01 & 320320400 & $\begin{array}{lll}5 & 5 & 5\end{array}$ & 646480 \\
\hline G7.5wt-02M & 7.5 & -0.02 & 320320400 & 10105 & 323280 \\
\hline G7.5wt-04M & 7.5 & -0.04 & 320320400 & 10105 & 323280 \\
\hline G5wt-02M & 5.0 & -0.02 & 320320400 & 10105 & 323280 \\
\hline G5wt-04M & 5.0 & -0.04 & 320320400 & $1010 \quad 5$ & 323280 \\
\hline G2.5wt-02M & 2.5 & -0.02 & 320320400 & 10105 & 323280 \\
\hline G2.5wt-01M & 2.5 & -0.01 & 320320400 & 10105 & 323280 \\
\hline G2.5wt-04M & 2.5 & -0.04 & 320320400 & 10105 & 323280 \\
\hline G1.5wt-02M & 1.5 & -0.02 & 320320400 & 10105 & 323280 \\
\hline G10.4wt-06M & 10.4 & -0.06 & 320320400 & 10105 & 323280 \\
\hline G10.4wt-02T & 10.4 & -0.02 & 640640400 & $2020 \quad 5$ & 323280 \\
\hline G10.4wt-04T & 10.4 & -0.04 & 640640400 & 20205 & 323280 \\
\hline G5.2wt-02T & 5.2 & -0.02 & 640640400 & $2020 \quad 5$ & 323280 \\
\hline G2.6wt-02T & 2.6 & -0.02 & 640640400 & $2020 \quad 5$ & 323280 \\
\hline G5wt-02N $\ddagger$ & 5.0 & -0.02 & 480480480 & 15156 & 323280 \\
\hline
\end{tabular}

$\dagger$ Name denotes two forcings: 'G' for geostrophic wind speed and 'wt' for specified cooling (negative surface heat flux); suffix indicates grids other than the reference grid where $\mathrm{F}=$ fine, $\mathrm{M}=$ moderate, and $\mathrm{T}=$ test grids.

$\ddagger$ suffix $N$ denotes flow from the north, rather than from the west as in other simulations. 
Table 6.2: SBL Characteristics for Different Simulation Forcings.

\begin{tabular}{|l|c|c|c|c|c|c||}
\hline Namet & $\begin{array}{c}B_{0} \\
\times 10^{3} \\
\left(\mathrm{~m}^{2} / \mathrm{s}^{3}\right)\end{array}$ & $\begin{array}{c}B_{0} \\
G^{2}|f|\end{array}$ & $\begin{array}{c}\text { Friction } \\
\text { Velocity } \\
(\mathrm{m} / \mathrm{s})\end{array}$ & $\begin{array}{c}\text { SBL } \\
\text { depth } \\
(\mathrm{m})\end{array}$ & $\begin{array}{c}\text { inversion } \\
\text { strength } \\
(\mathrm{K} / 100 \mathrm{~m})\end{array}$ & $\begin{array}{c}\text { low level } \\
\text { jet speed } \\
(\mathrm{m} / \mathrm{s})\end{array}$ \\
\hline \hline G7.5wt-02 & 0.68 & 0.12 & 0.21 & 170 & 3.1 & 9.8 \\
\hline G5wt-02 & 0.68 & 0.27 & 0.11 & 70 & 18.0 & 7.1 \\
\hline G10wt-02 & 0.68 & 0.07 & 0.33 & 310 & 0.9 & 10.6 \\
\hline G2.5wt-02M & 0.68 & 1.08 & 0.05 & 60 & 34.5 & 3.6 \\
\hline G1.5wt-02M & 0.68 & 3.00 & 0.04 & 40 & 53.0 & 1.9 \\
\hline G2.5wt-04M & 1.35 & 2.16 & 0.07 & 40 & 110.0 & 3.4 \\
\hline G5wt-01 & 0.34 & 0.14 & 0.12 & 80 & 4.9 & 7.0 \\
\hline G10.4wt-06M & 2.03 & 0.19 & 0.22 & 130 & 17.9 & 13.3 \\
\hline
\end{tabular}

$\dagger$ Name denotes two forcings: 'G' for geostrophic wind speed and 'wt' for specified cooling (negative surface heat flux); suffix indicates grid other than the reference grid where $\mathrm{M}=$ moderate grid. 


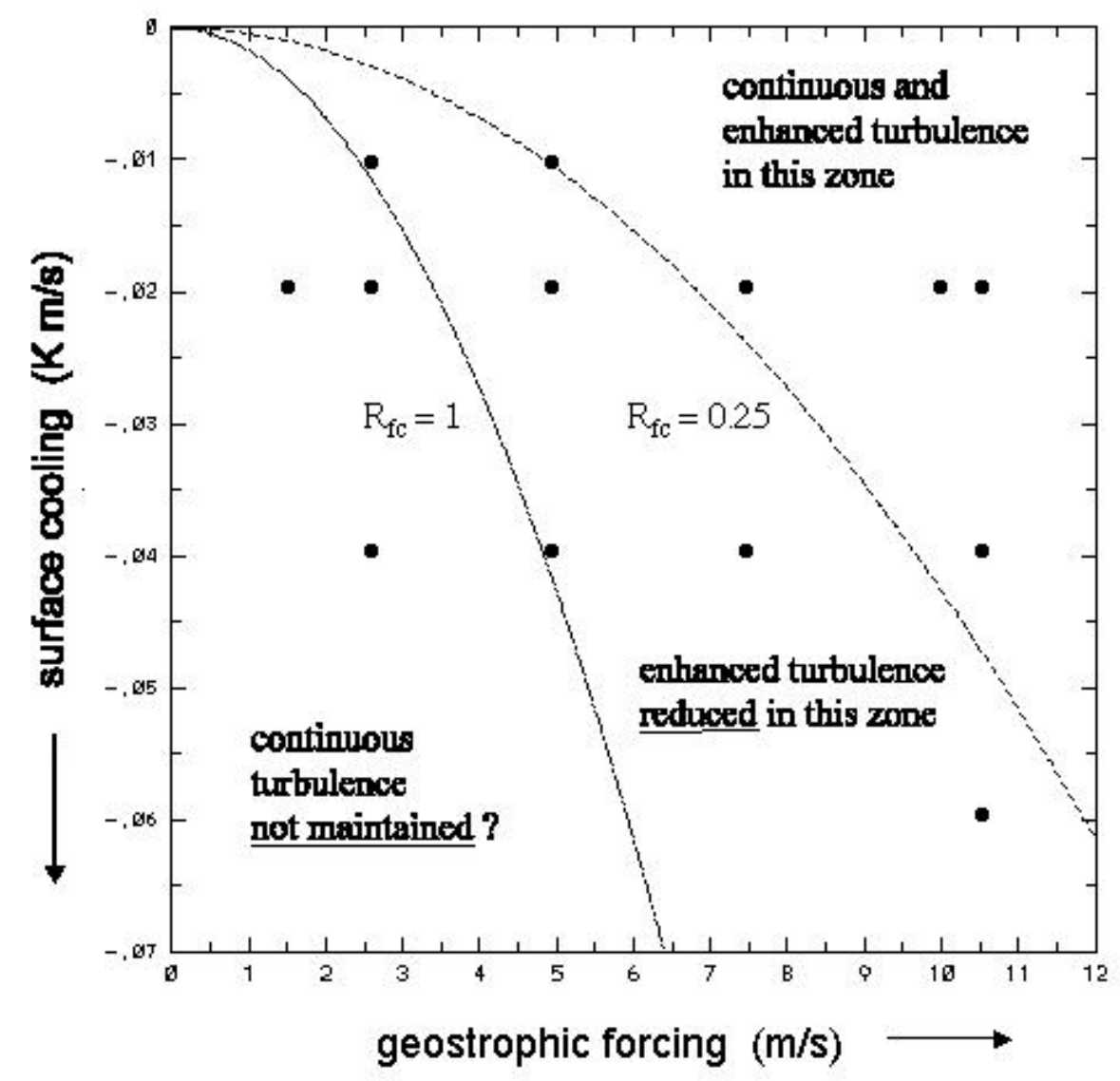

Figure 6.1: Simulation forcings used and two different criteria that specify limiting forcings that support turbulence; turbulence zones indicated; solid circles show simulation parameter pairings. 

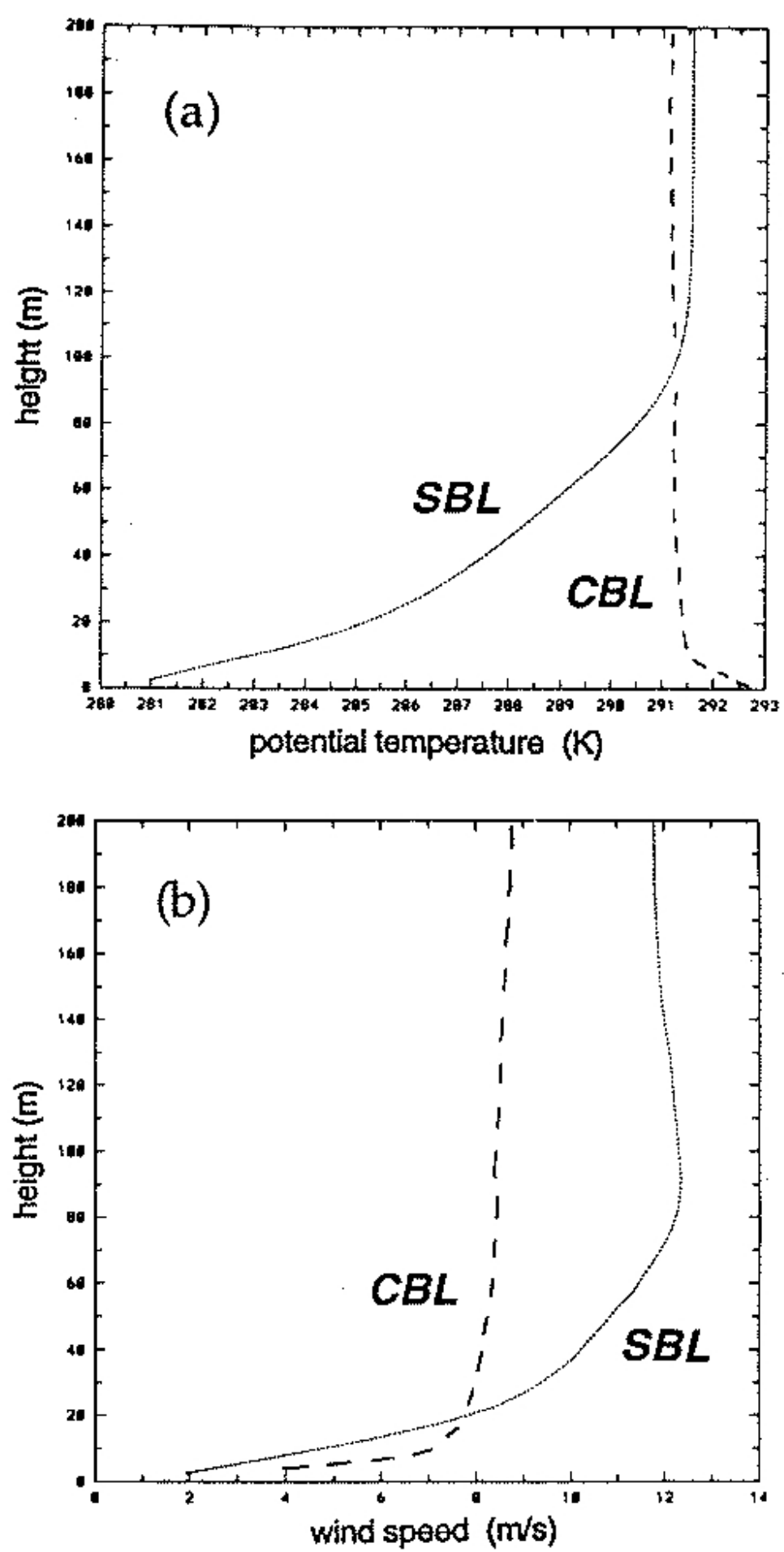

Figure 6.2: Vertical profiles of (a) simulated temperature and (b) winds in the lowest 200 $\mathrm{m}$ of the SBL and CBL for Run G10.4wt-02T. 

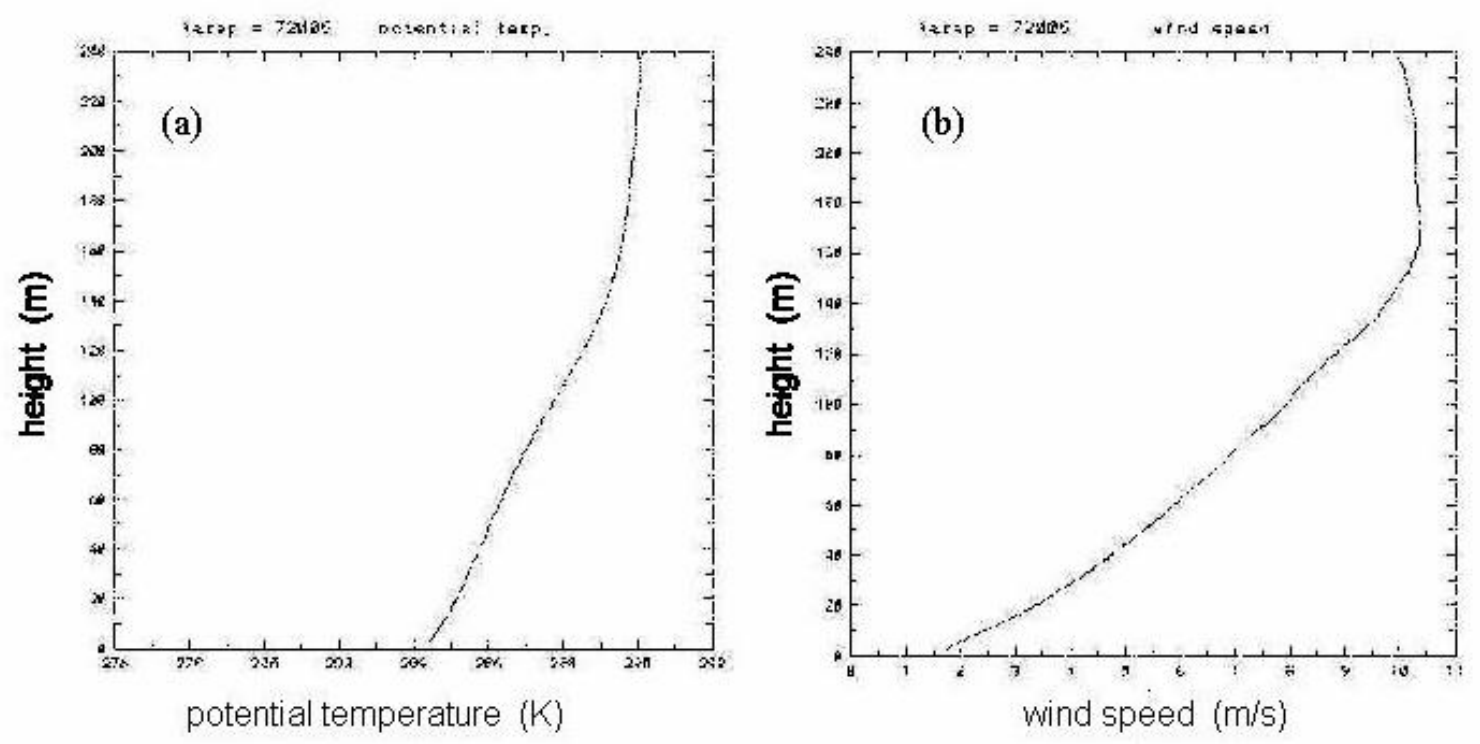

Figure 6.3: Vertical profiles of simulated (a) potential temperature and (b) wind speed for Run G7.5wt-02 at hour 9.
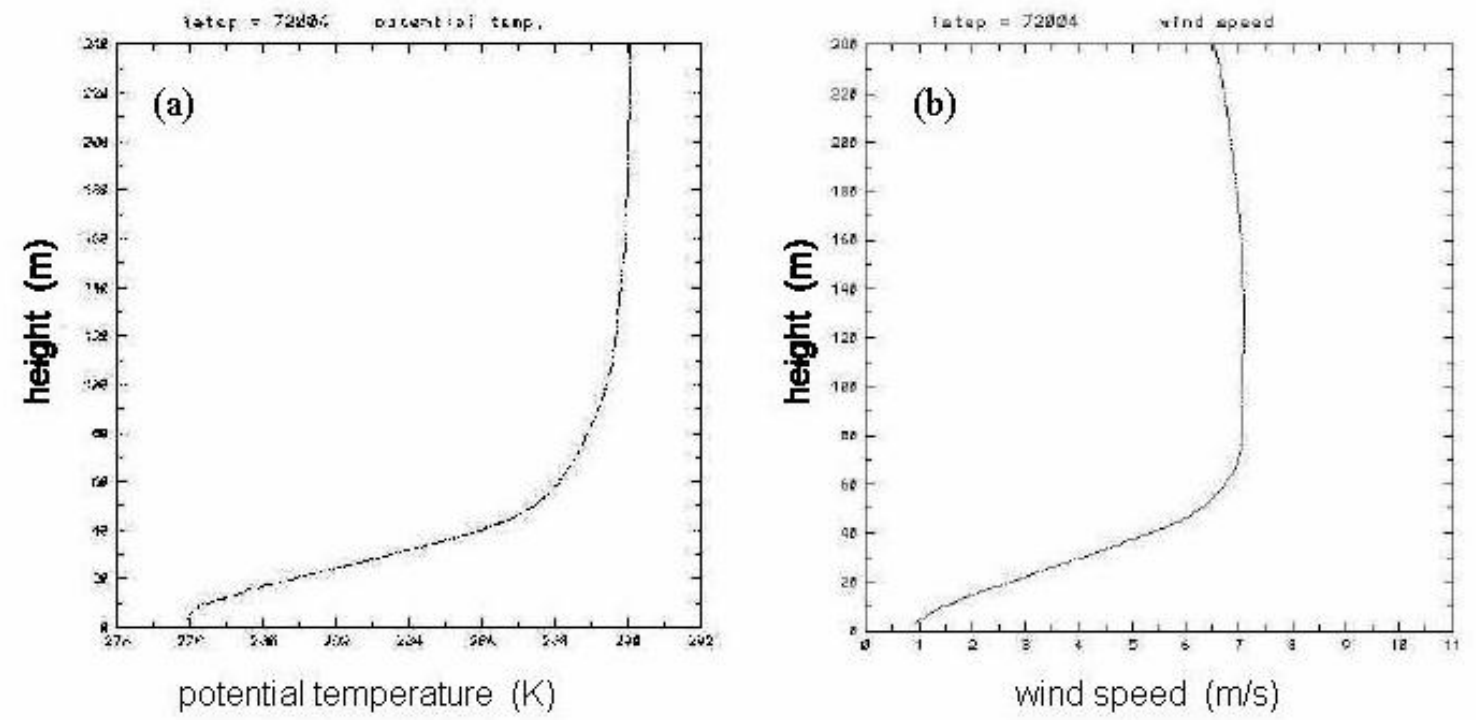

Figure 6.4: Vertical profiles of Vertical profiles of simulated (a) potential temperature and (b) wind speed for Run G5wt-02 at hour 9. 


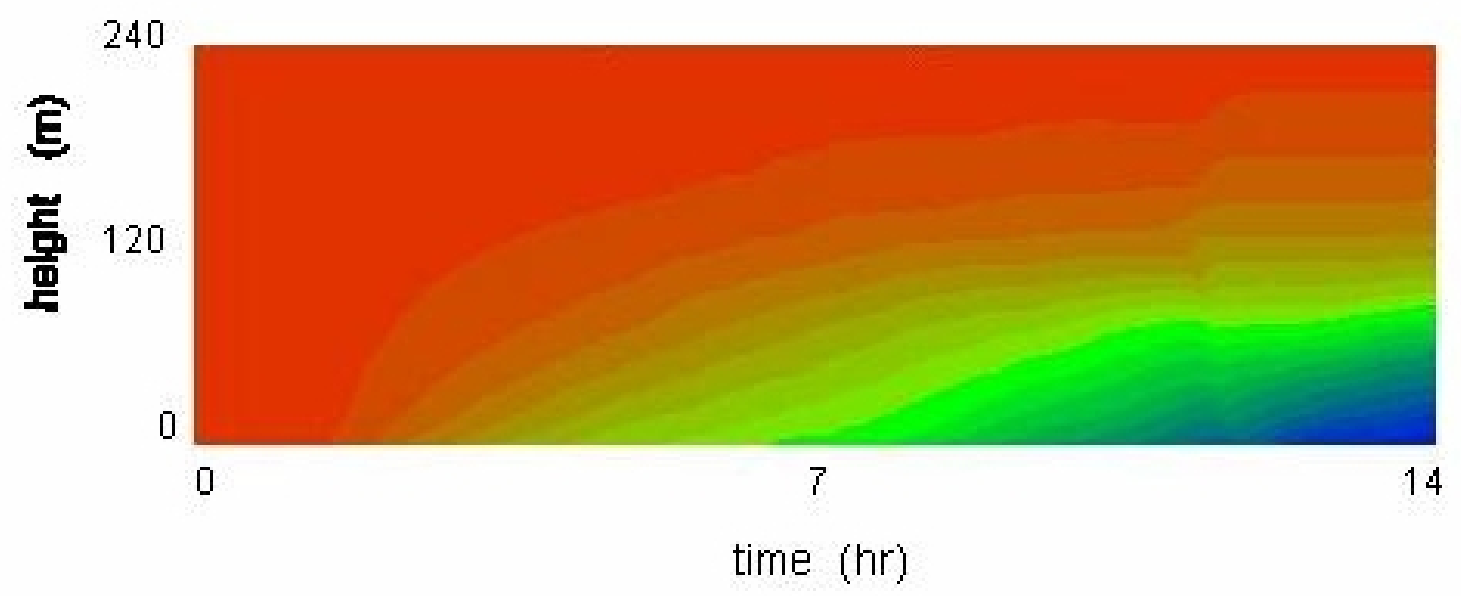

Figure 6.5: Time history of evolving temperature structure from NBL to SBL for Run G7.5wt-02; color range (blue to red) is $279-291 \mathrm{~K}$.

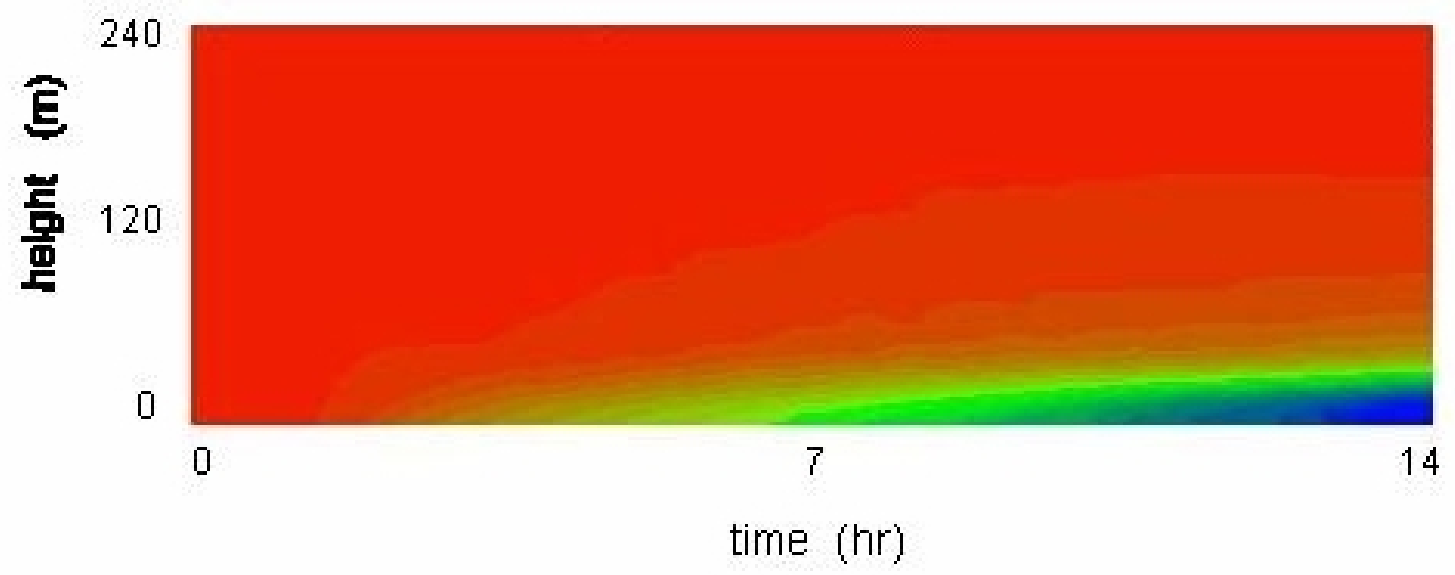

Figure 6.6: Time history of evolving temperature structure from NBL to SBL for Run G5wt-02; color range (blue to red) is $267-291 \mathrm{~K}$. 


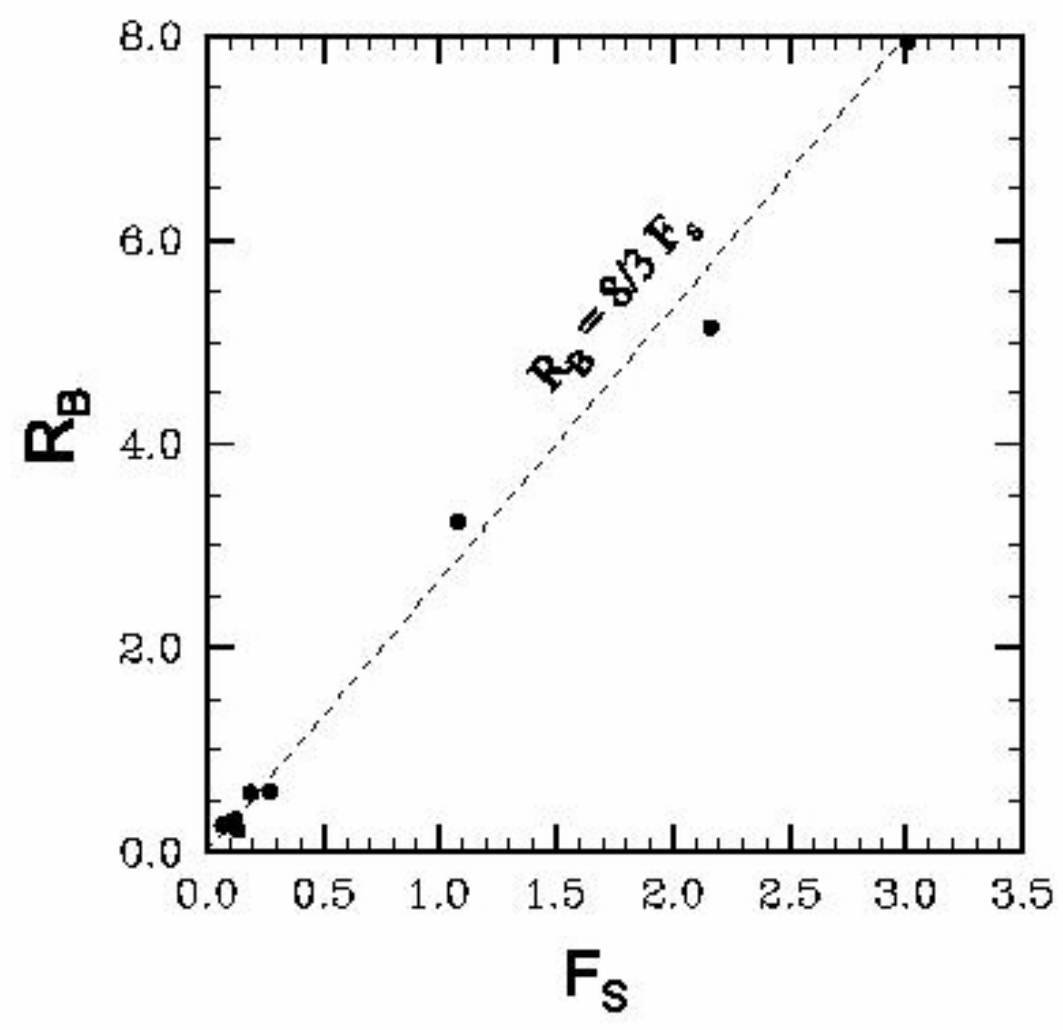

Figure 6.7: Stability forcing index $\left(F_{s}\right)$ and bulk Richardson number $\left(R_{B}\right)$ for resulting SBLs summarized in Table 6.2; line represents visual linear fit to points, and is defined by $R_{B}=8 / 3 F_{s}$. 


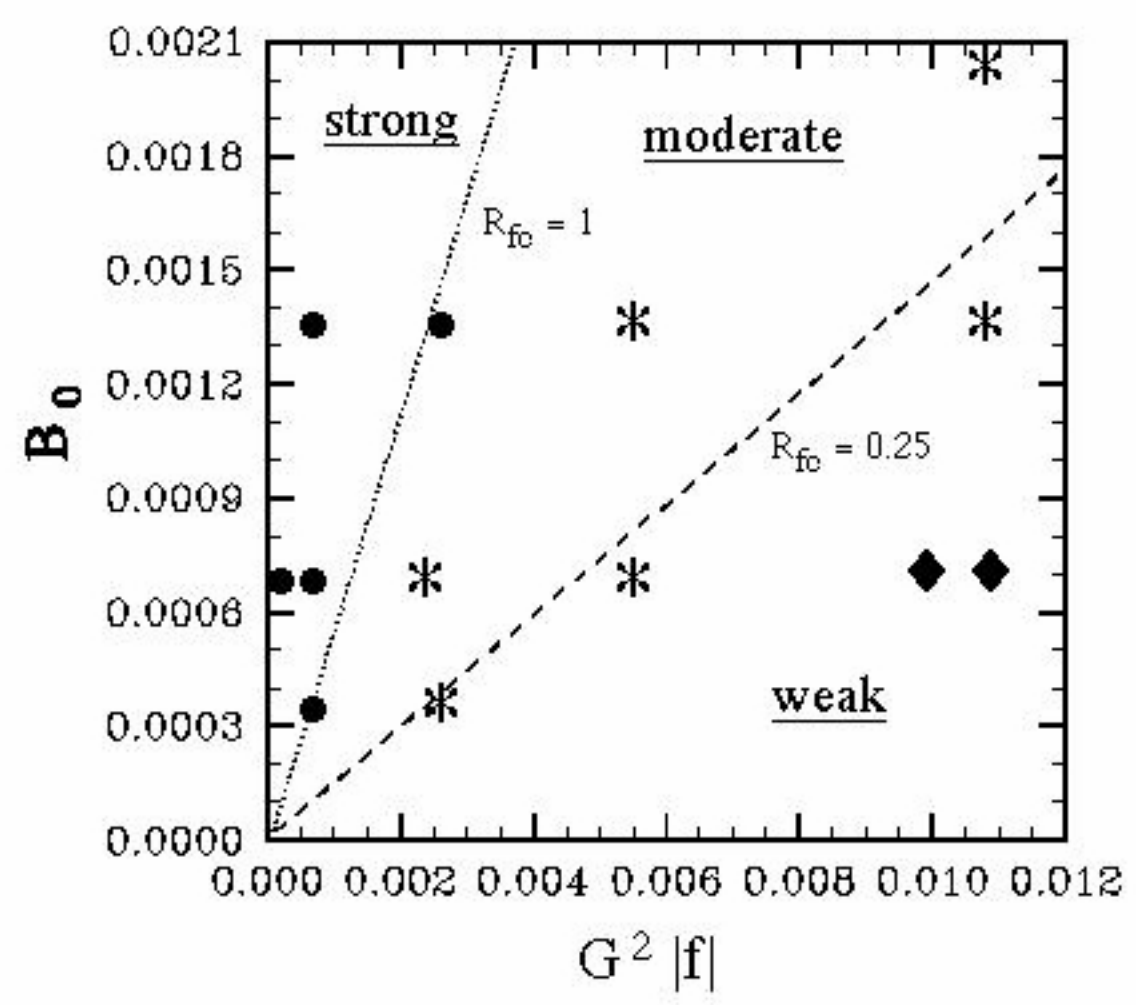

Figure 6.8: Simulation forcings in terms of $B_{0}$ and $G^{2}|f|$; symbols for forcing pairs determined by $R_{B}$ from Equation 6.10 where solid diamond is for $0<R_{B} \leq 0.25$, asterisk is for $0.25<R_{B} \leq 1$, and solid circle is for $R_{B}>1$; Derbyshire criterion using $R_{f c}=1$ (dotted line) and $R_{f c}=0.25$ (dashed line); strength of stability indicated in each zone. 


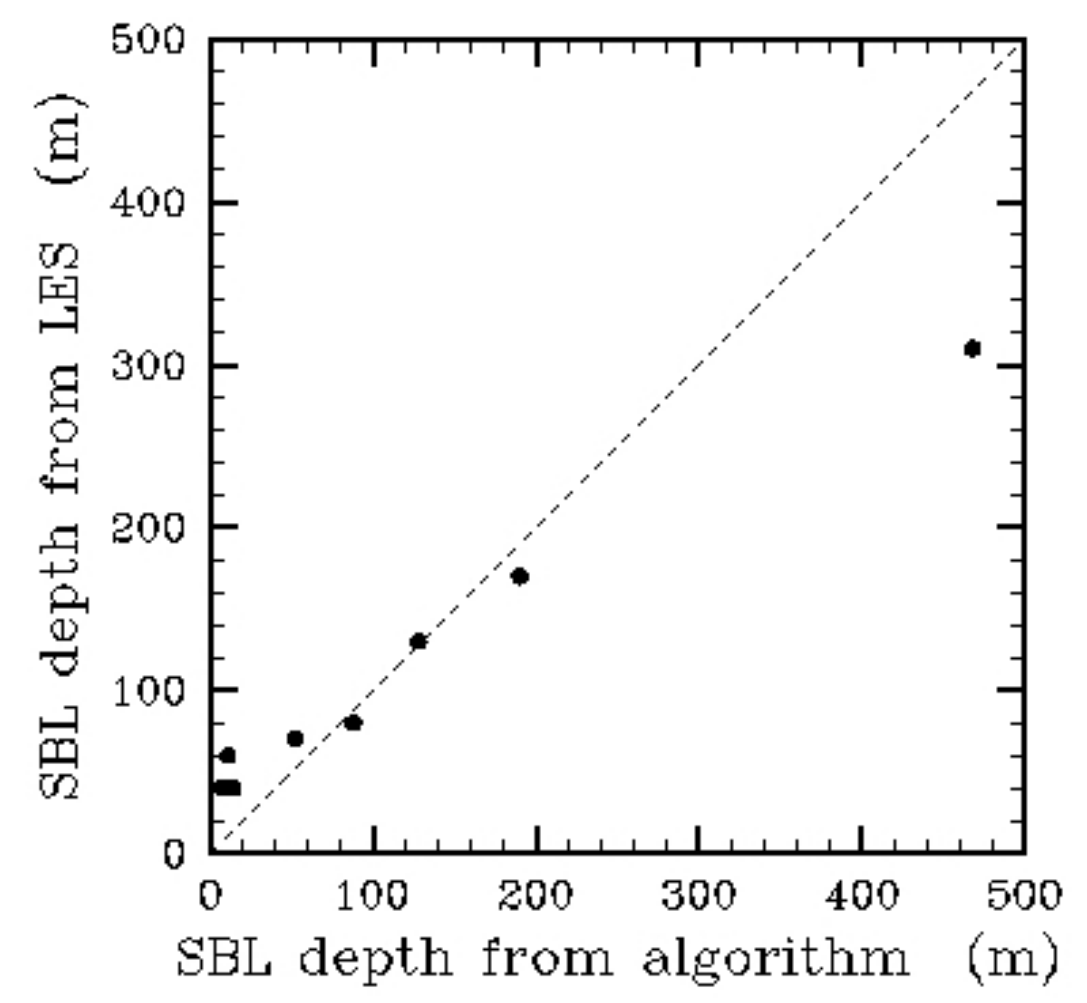

Figure 6.9: Evaluation of algorithm to an estimate the SBL depth, $h_{s}$, based on the friction velocity, $u_{*}$, and negative surface heat flux (in $W / m^{2}$ ), from Minnesota experiment: $h_{s}=21500 u_{*}^{2} / \sqrt{H}$. 
(a)
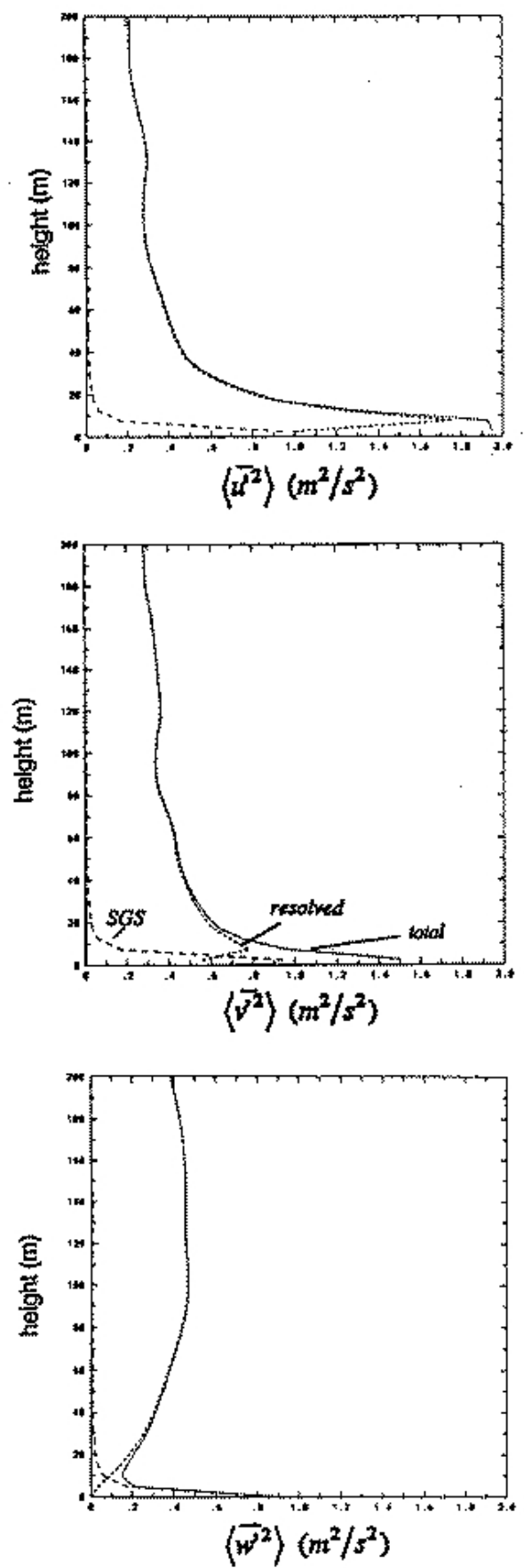

(b)
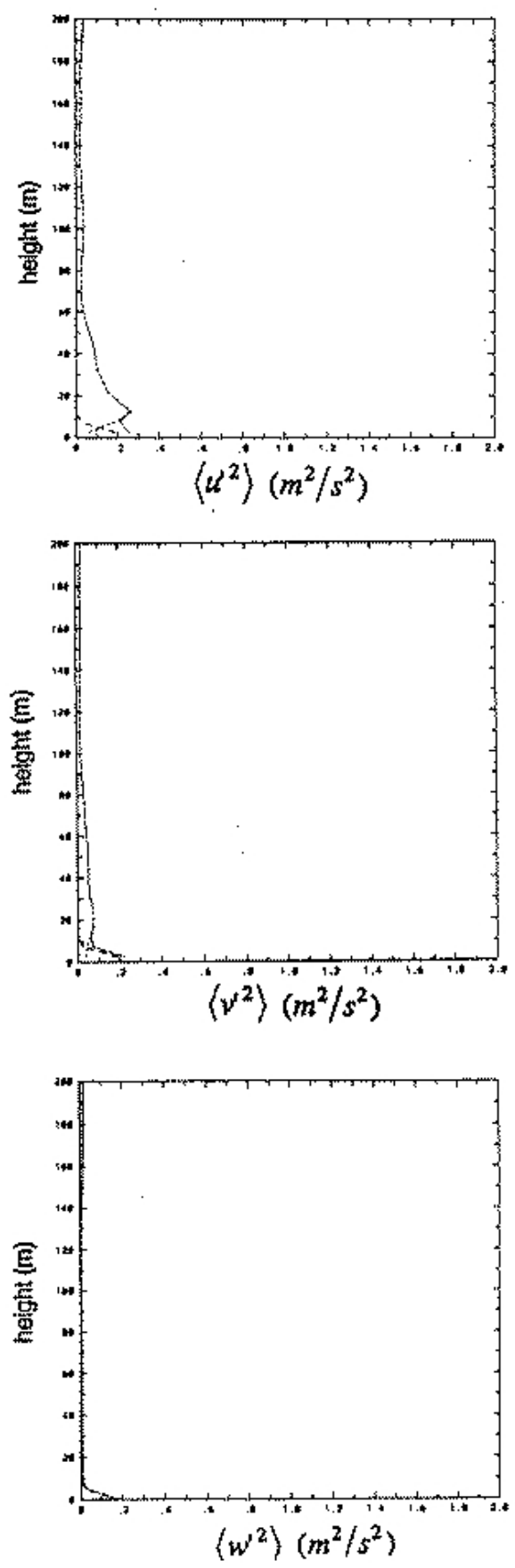

Figure 6.10: Vertical profiles of simulated velocity variances for (a) CBL and (b) SBL from Run G10.4wt-02T; the vertical axis is height $(0-200 \mathrm{~m})$, and the horizontal axis is velocity variance $\left(0-2 \mathrm{~m}^{2} / \mathrm{s}^{2}\right)$. 


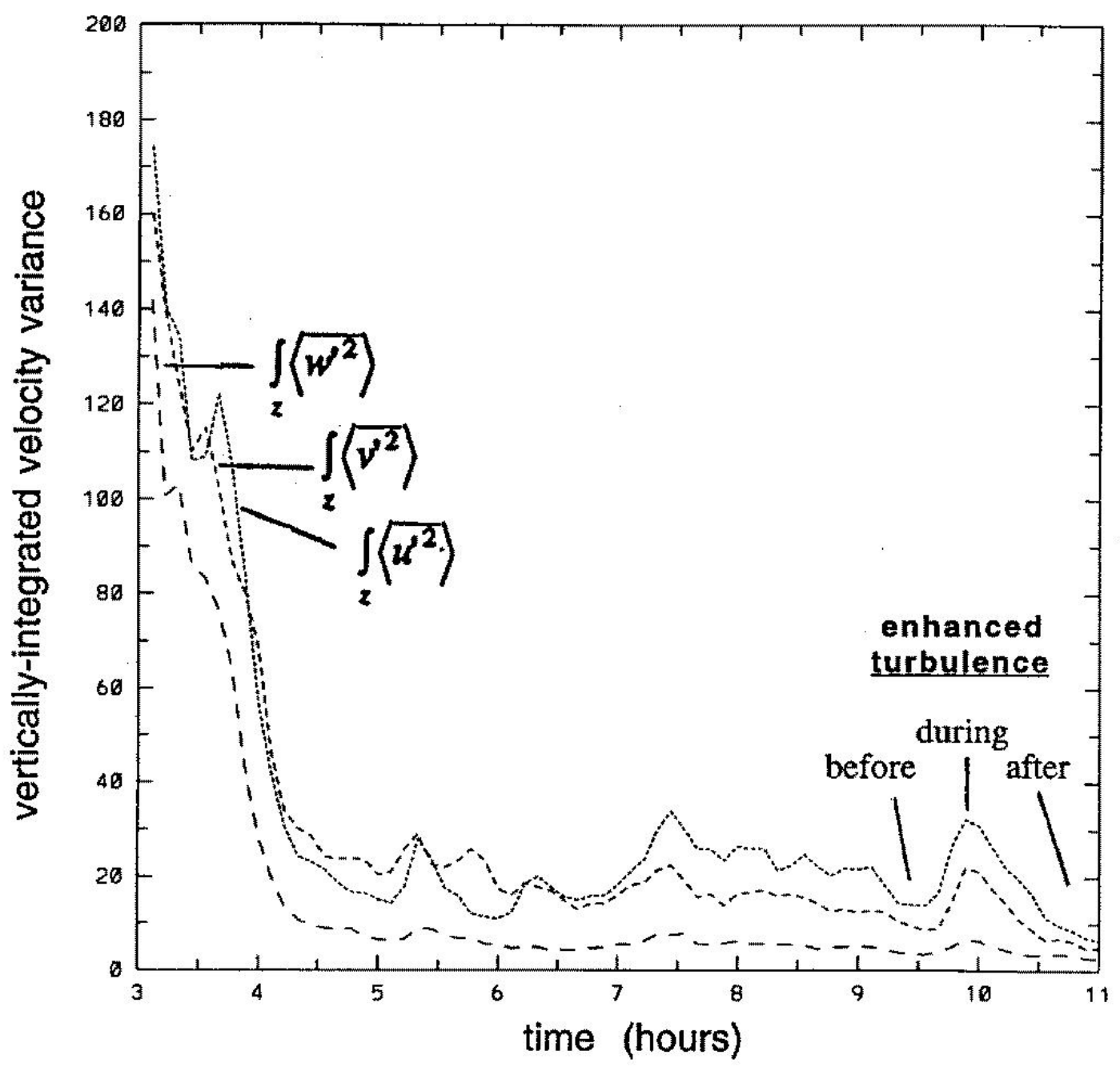

Figure 6.11: Time history of vertically-integrated velocity variances for LES generated wind fields in Run G10.4wt-02T. 


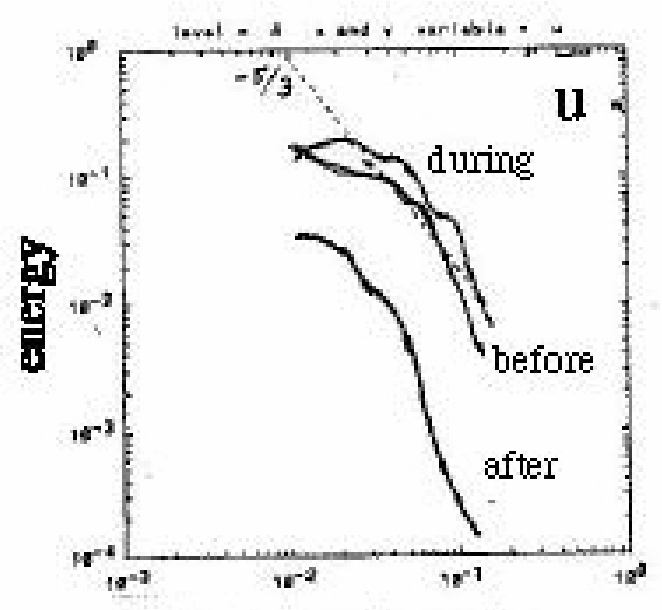

\section{(a)}

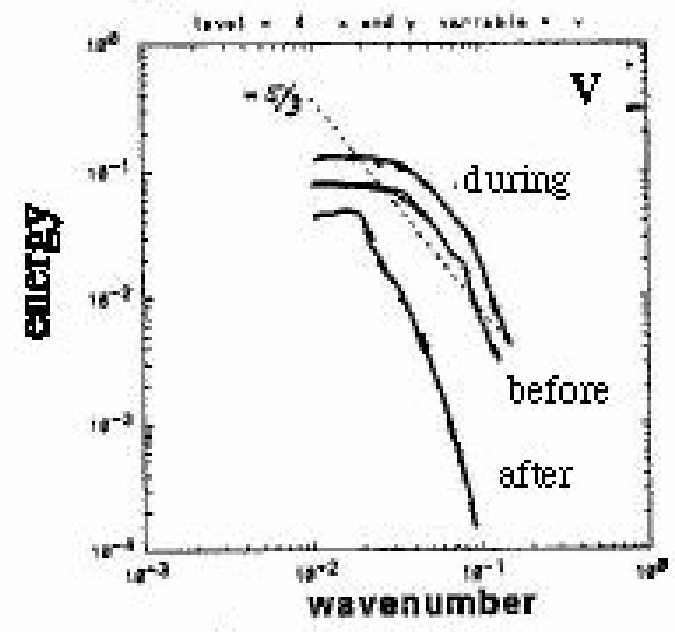

(b)

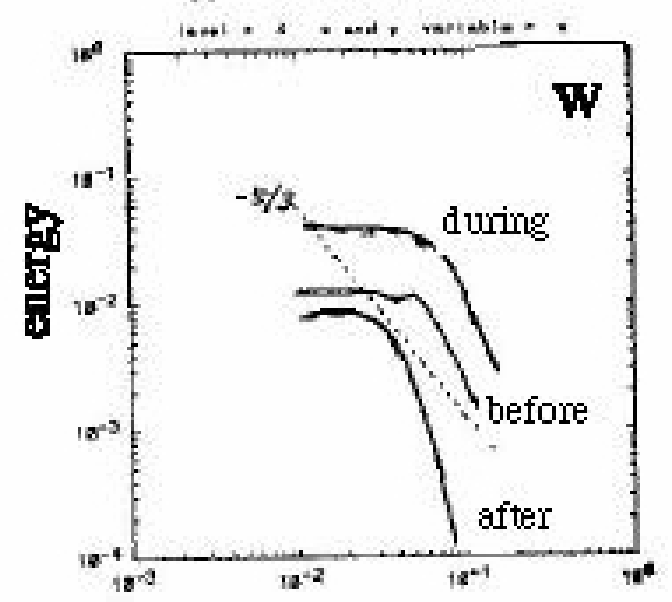

(c)

Figure 6.12: Spectra velocity variances before, during, and after SBL turbulence event for (a) u component, (b) v component, and (c) w component from Run G10.4wt-02T; horizontal axes are wavenumber and vertical axes are energy per wavenumber; $-5 / 3$ slope is indicated by dashed line in each plot. 

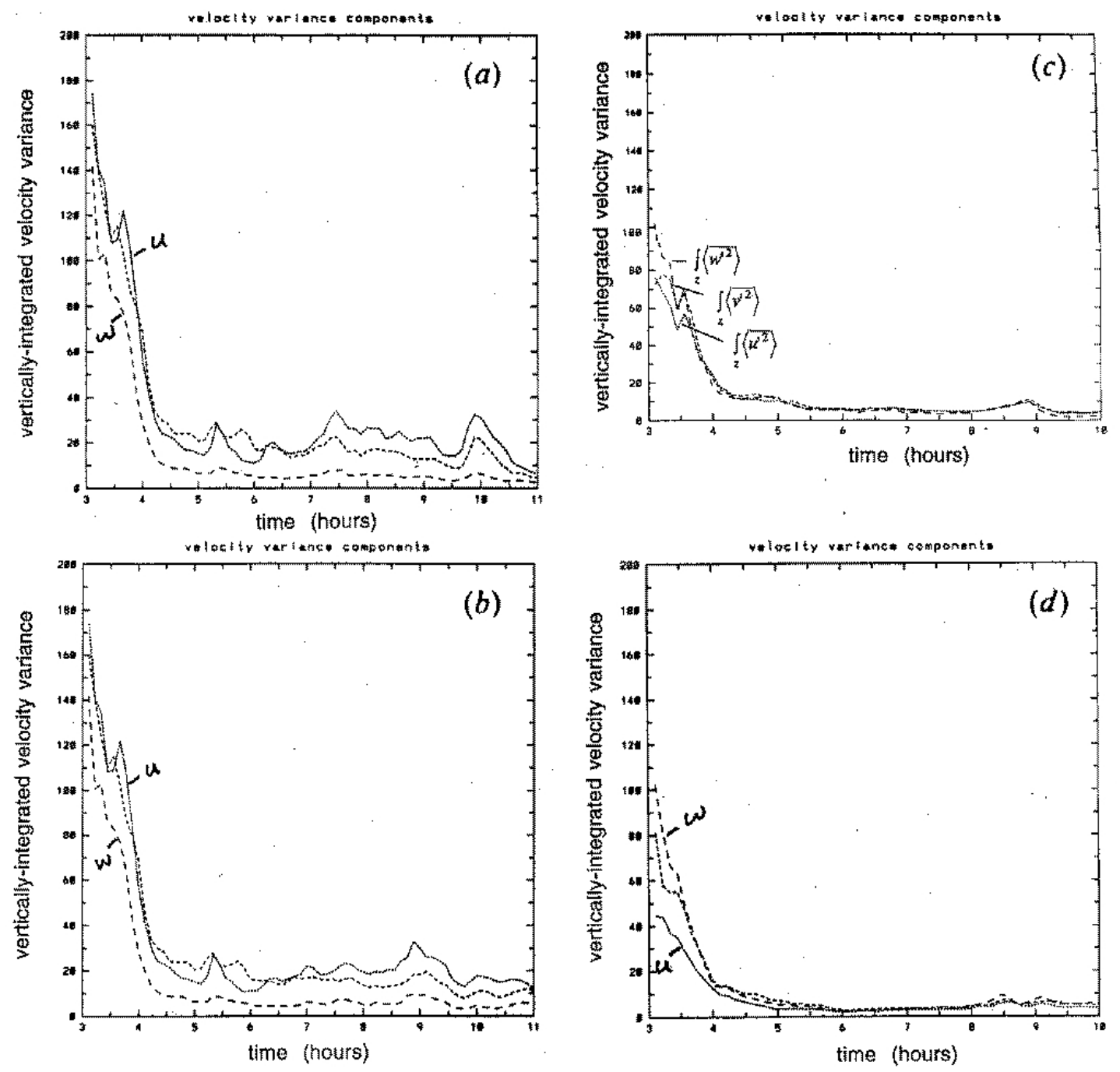

Figure 6.13: Time history of vertically-integrated velocity variances for LES generated wind fields for 4 cases: (a) Run G10.4wt-02T also in Figure 6.11, (b) Run G10.4wt-04T, (c) Run G2.6wt-02T, and (d) Run G5.2wt-02T. 


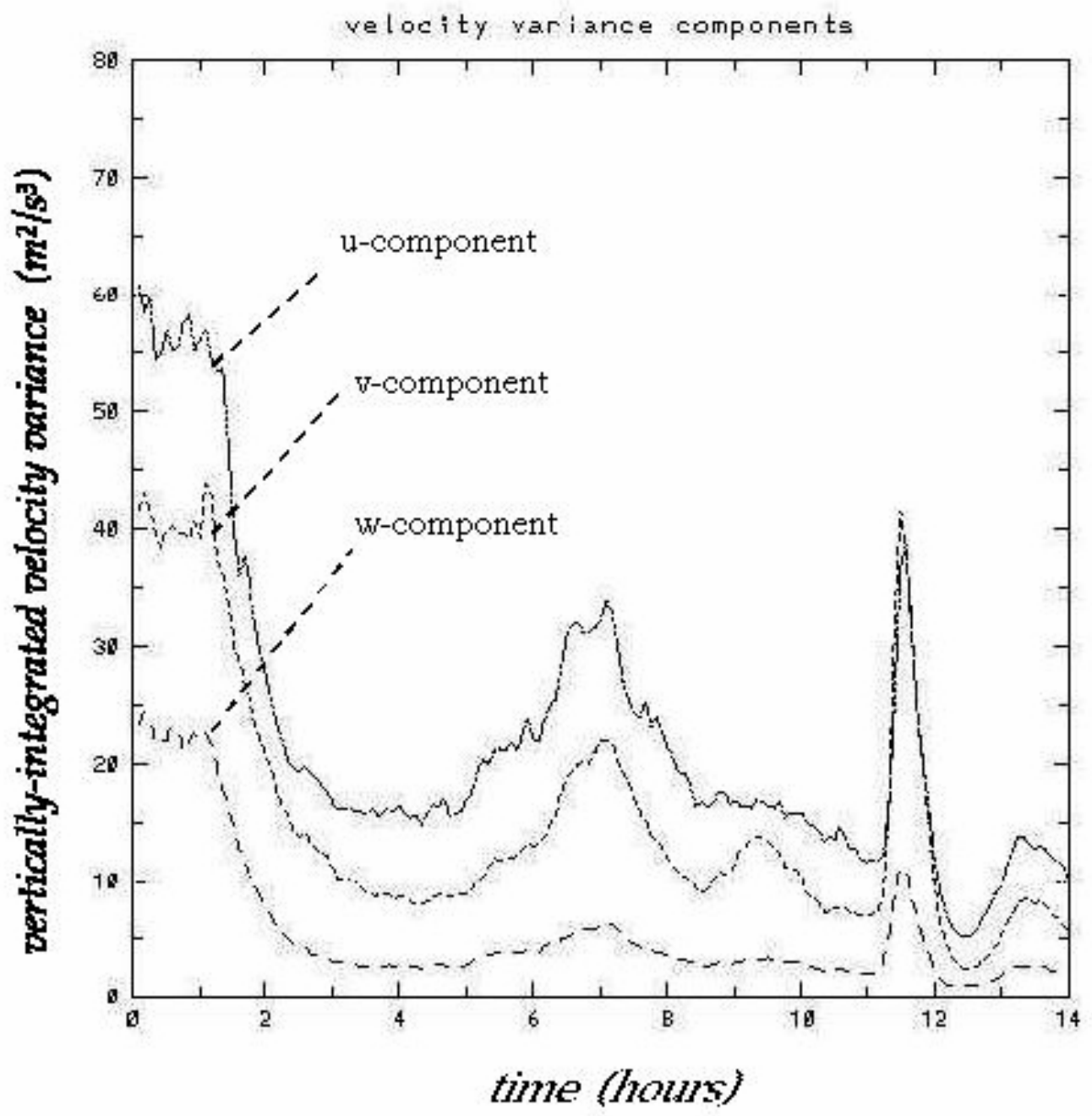

Figure 6.14: Time history of vertically-integrated velocity variances for LES generated wind fields in Run G7.5wt-02. 


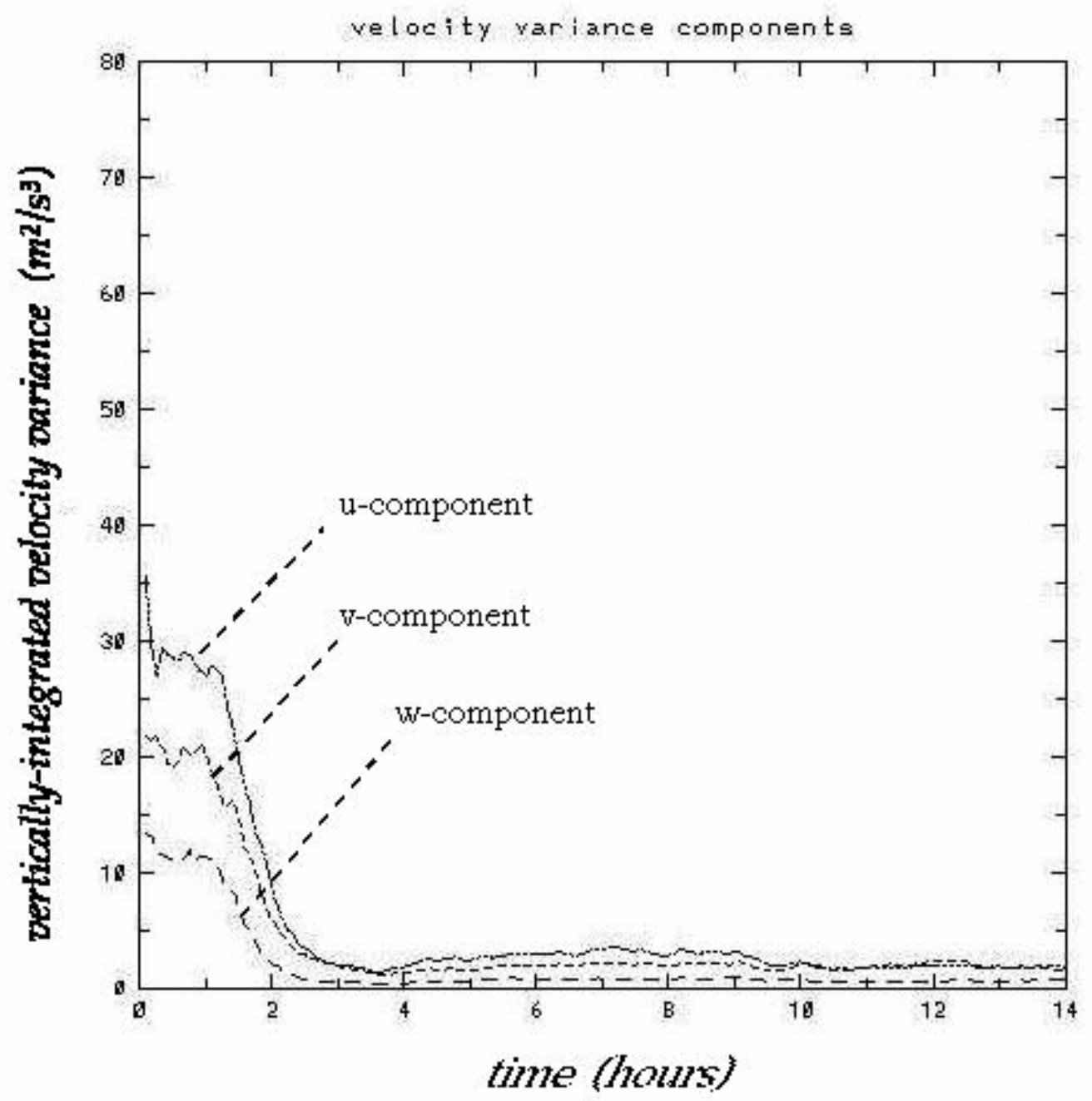

Figure 6.15: Time history of vertically-integrated velocity variances for LES generated wind fields in Run G5wt-02. 


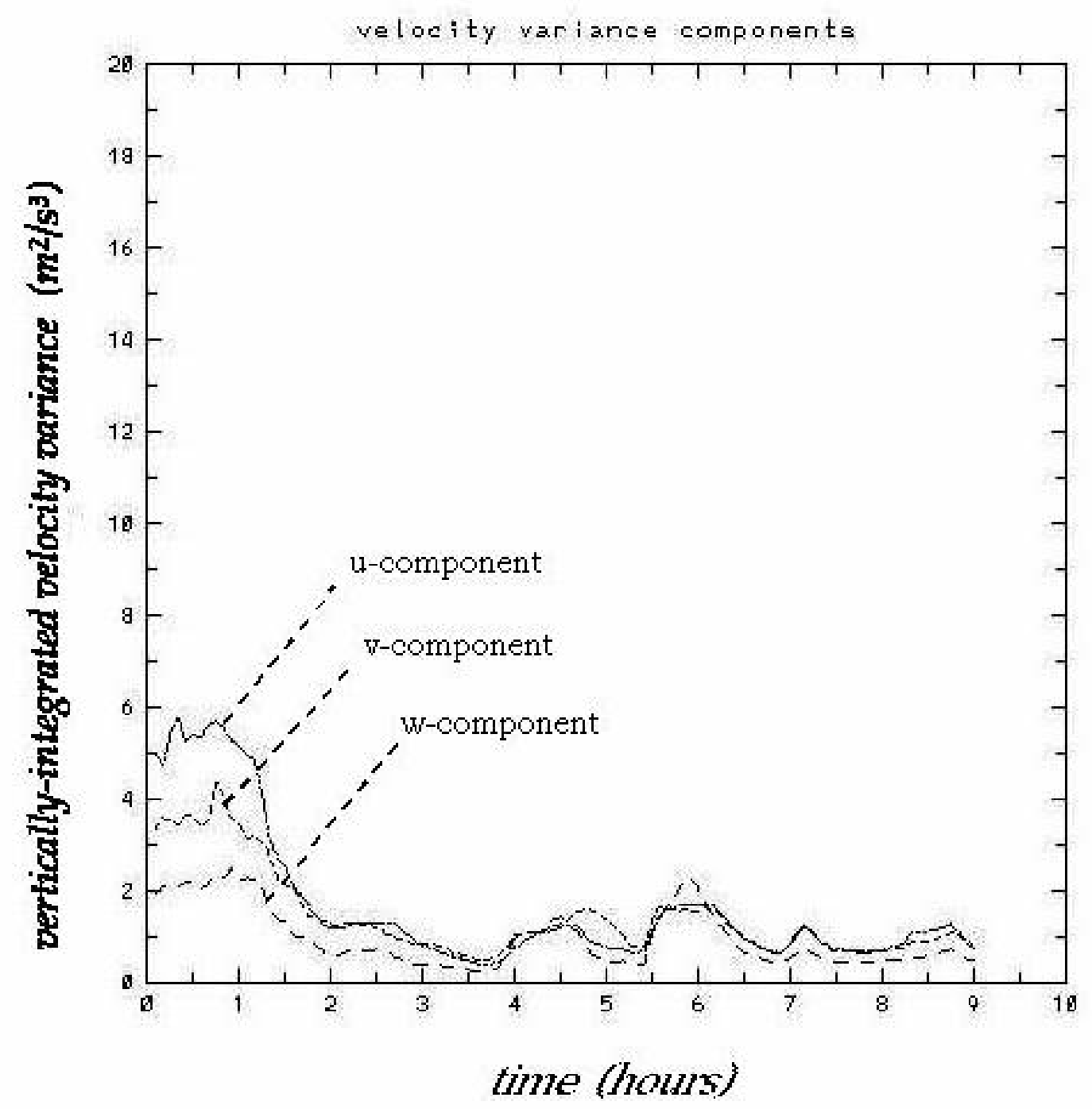

Figure 6.16: Time history of vertically-integrated velocity variances for LES generated wind fields in Run G2.5wt-04. 


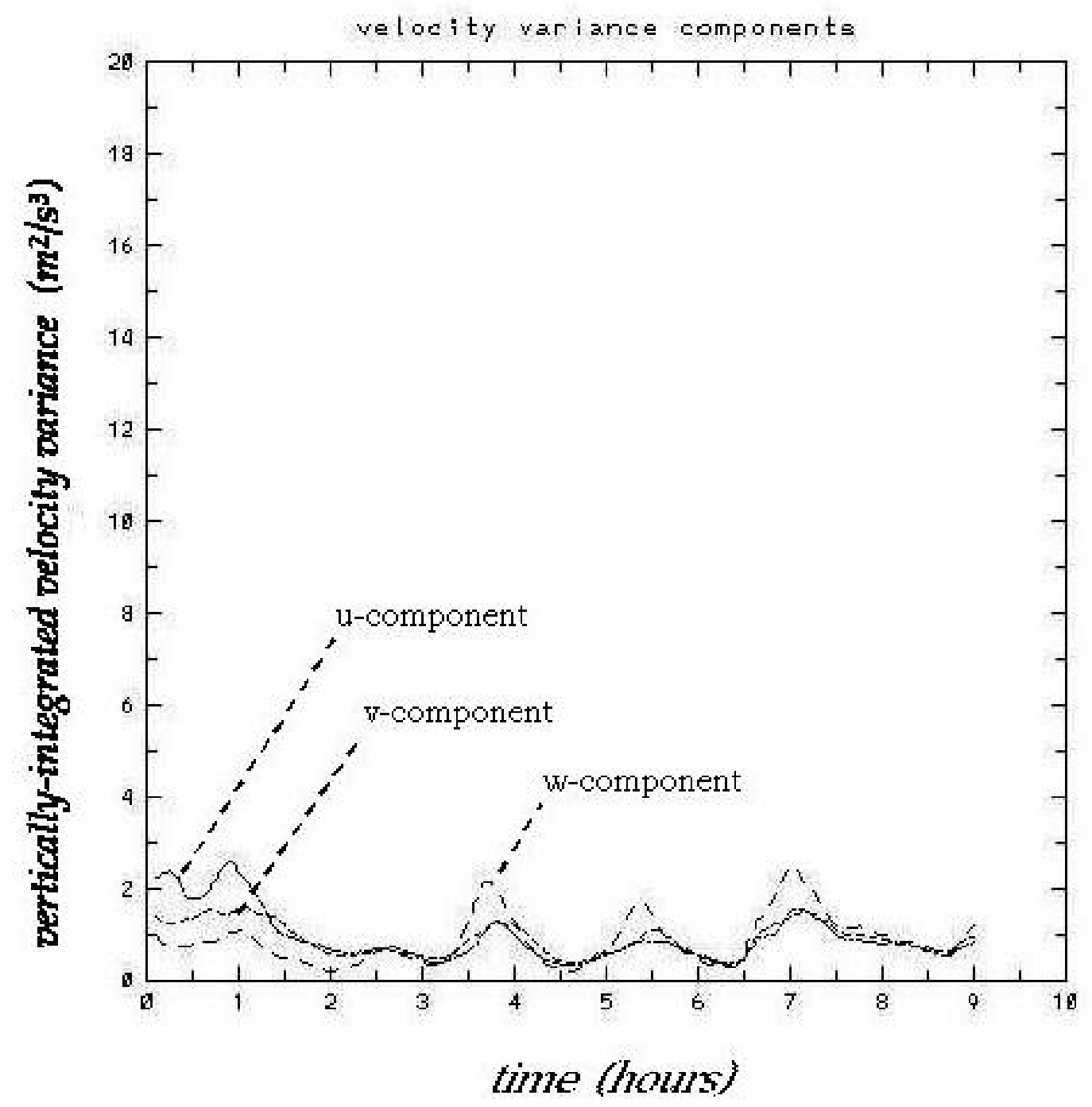

Figure 6.17: Time history of vertically-integrated velocity variances for LES generated wind fields in Run G1.5wt-02. 
(a)
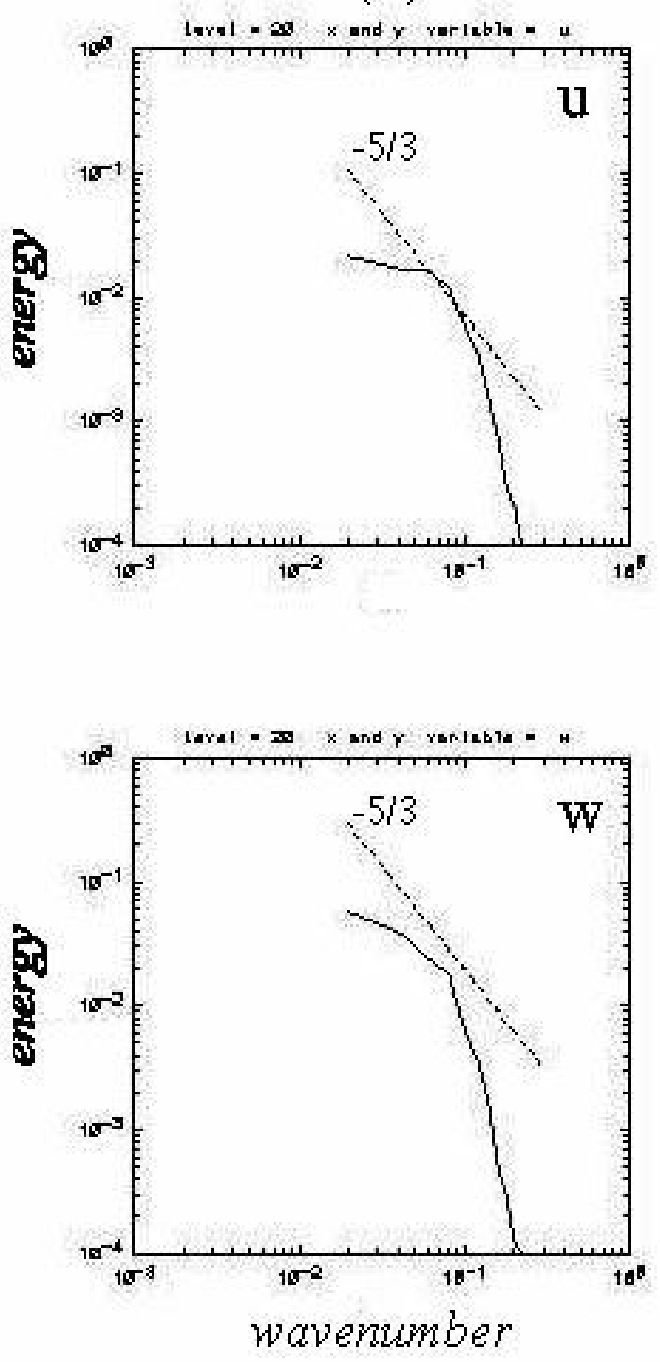

(b)
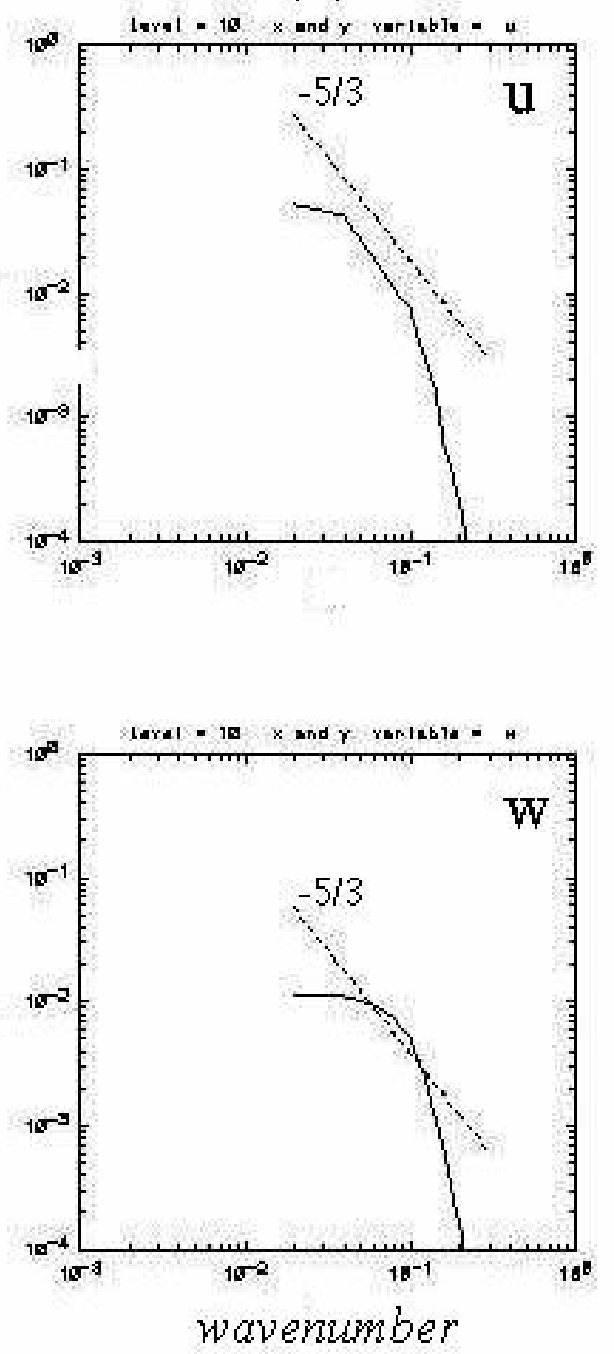

Figure 6.18: spectra of $\mathrm{u}$ and $\mathrm{w}$ velocity variances for Run G1.5wt-02 at hour 7 at heights of (a) $92.5 \mathrm{~m}$ for $\mathrm{u}$ and $90 \mathrm{~m}$ for $\mathrm{w}$, and (b) $42.5 \mathrm{~m}$ for $\mathrm{u}$ and $40 \mathrm{~m}$ for $\mathrm{w}$; dashed line indicates $-5 / 3$ slope. 


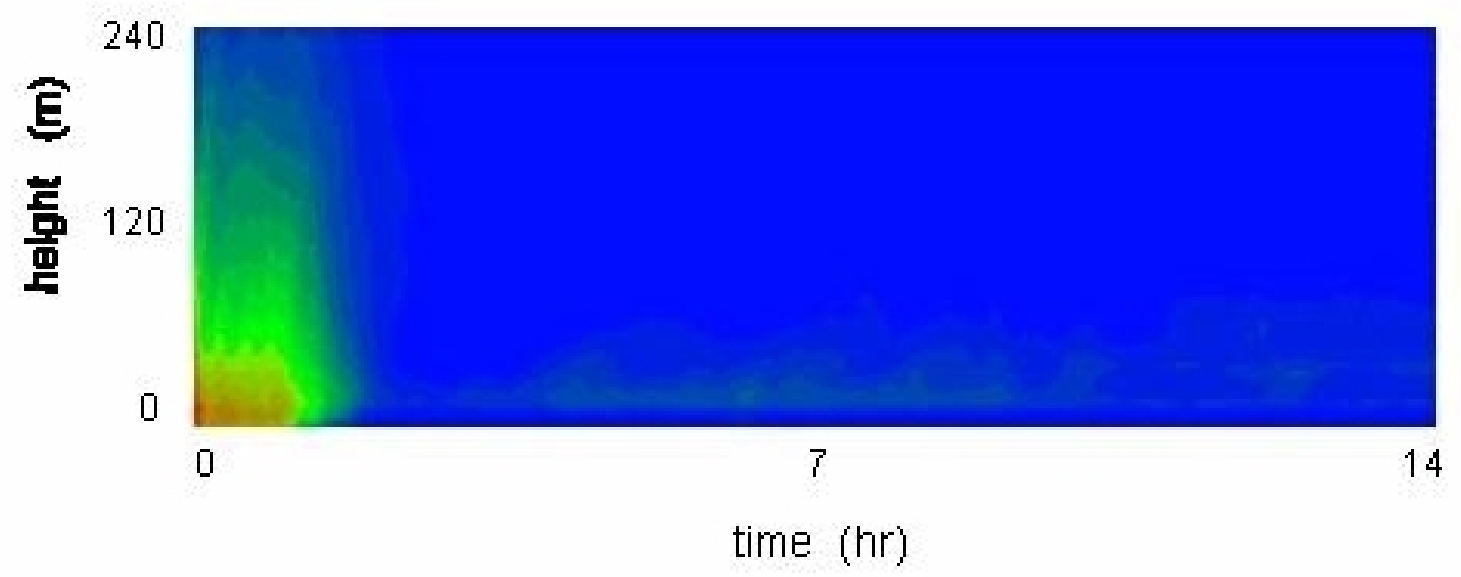

Figure 6.19: Time history of evolving TKE structure from NBL to SBL for Run G7.5wt-02; color range (blue to red) is $0-50 \mathrm{~m}^{2} / \mathrm{s}^{2}$.

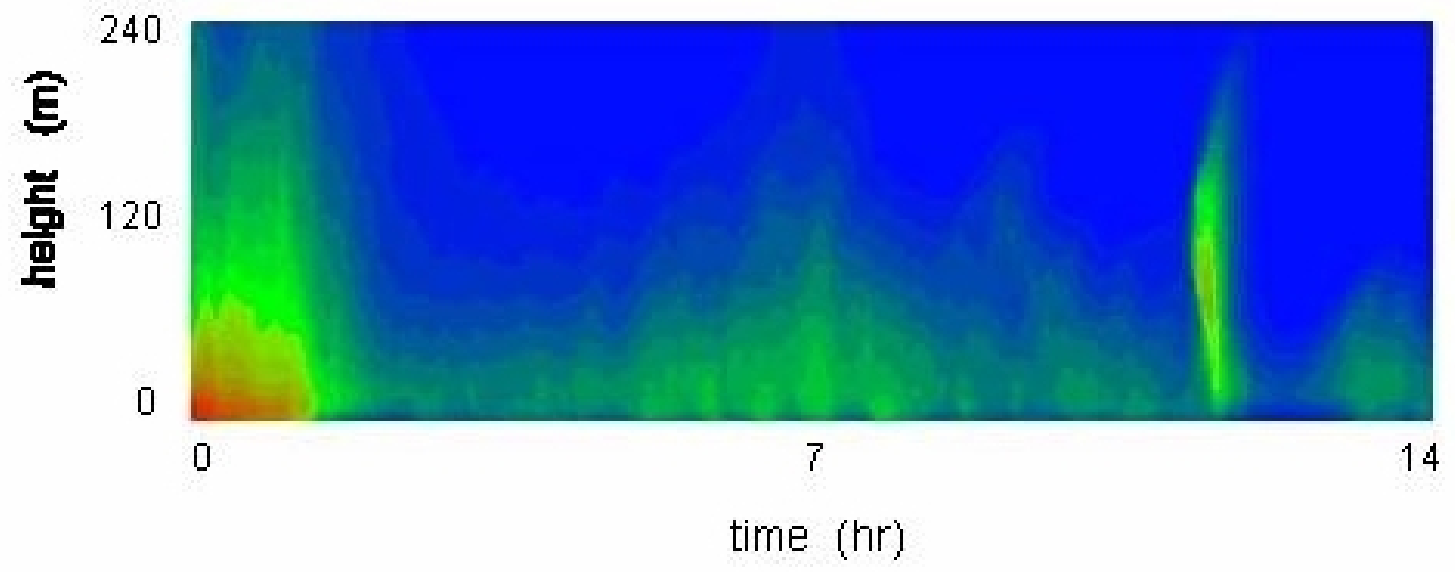

Figure 6.20: Time history of evolving TKE structure from NBL to SBL for Run G5wt-02; color range (blue to red) is $0-30 \mathrm{~m}^{2} / \mathrm{s}^{2}$. 


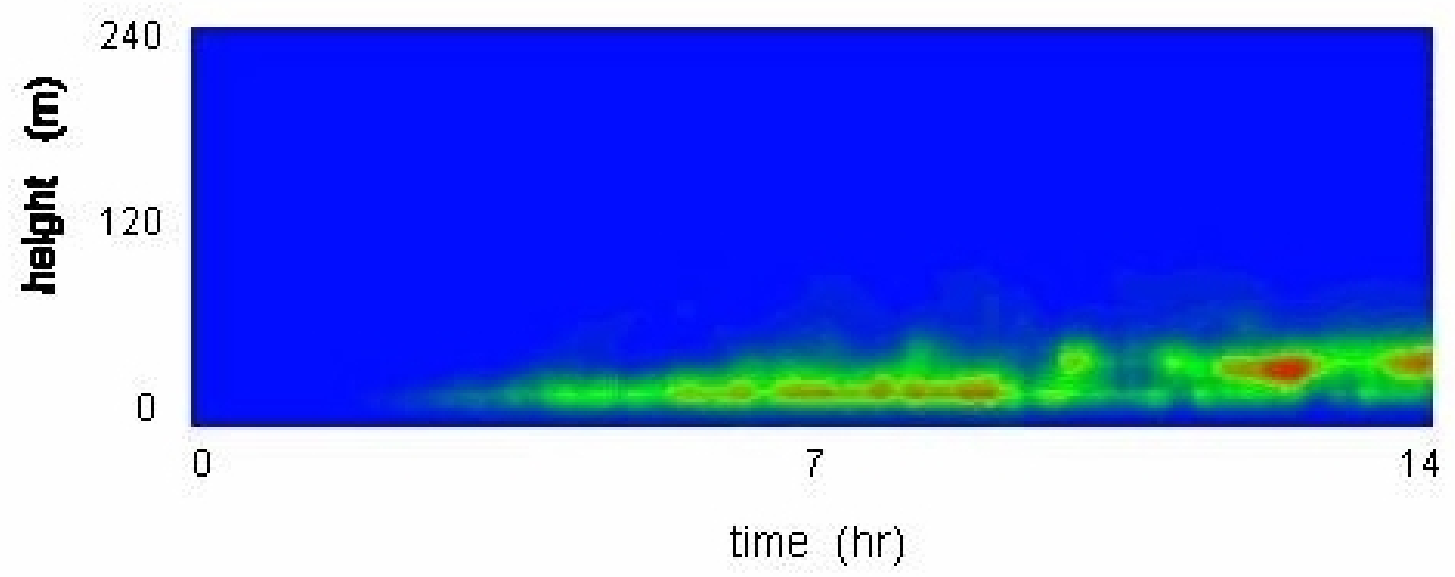

Figure 6.21: Time history of evolving temperature variance structure from NBL to SBL for Run G7.5wt-02; color range (blue to red) is $0-30 \mathrm{~K}^{2}$.

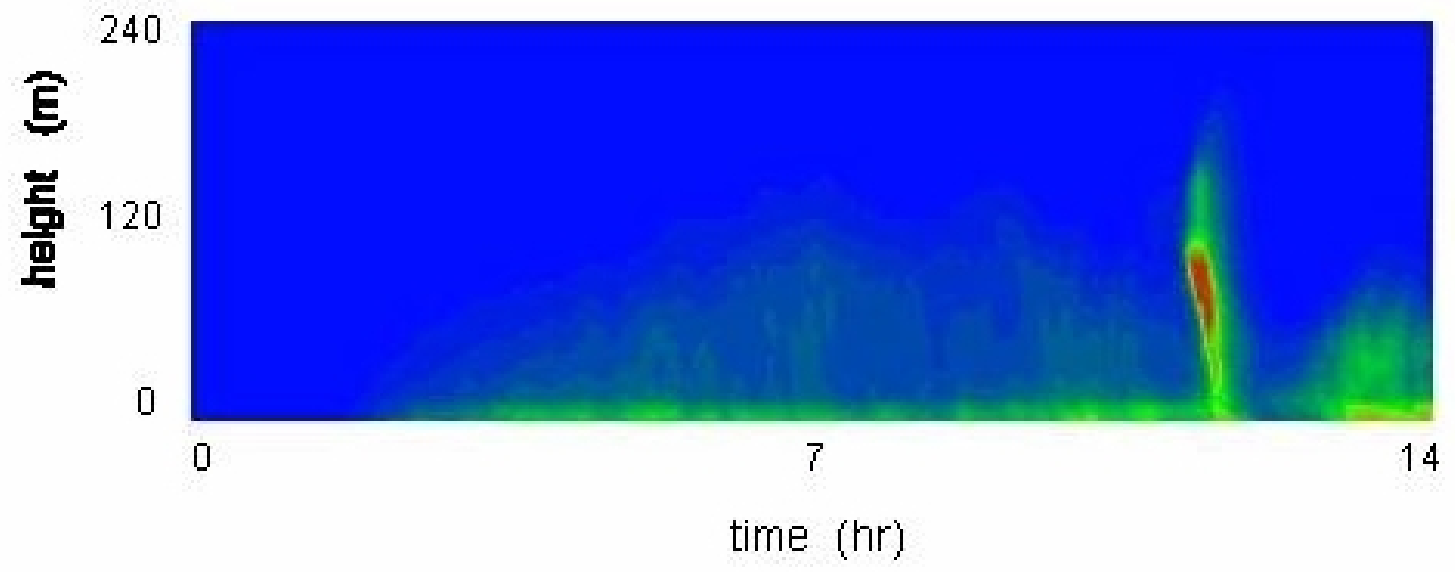

Figure 6.22: Time history of evolving temperature variance structure from NBL to SBL for Run G5wt-02; color range (blue to red) is $0-40 \mathrm{~K}^{2}$. 


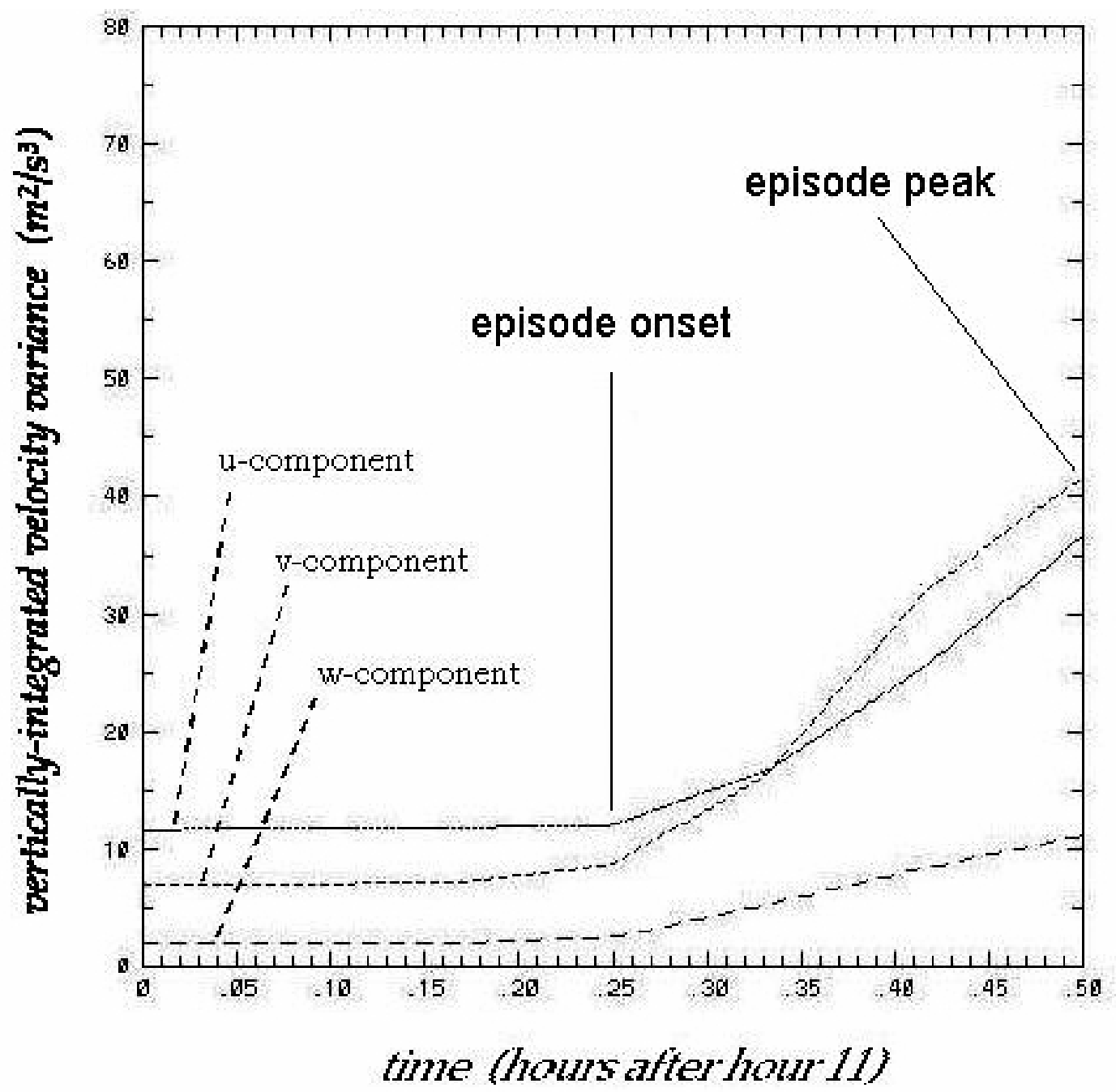

Figure 6.23: Time history of vertically-integrated velocity variances for LES generated wind fields in Run G7.5wt-02 for episode of enhanced turbulence; plot is for the 30 minutes following hour 11 . 


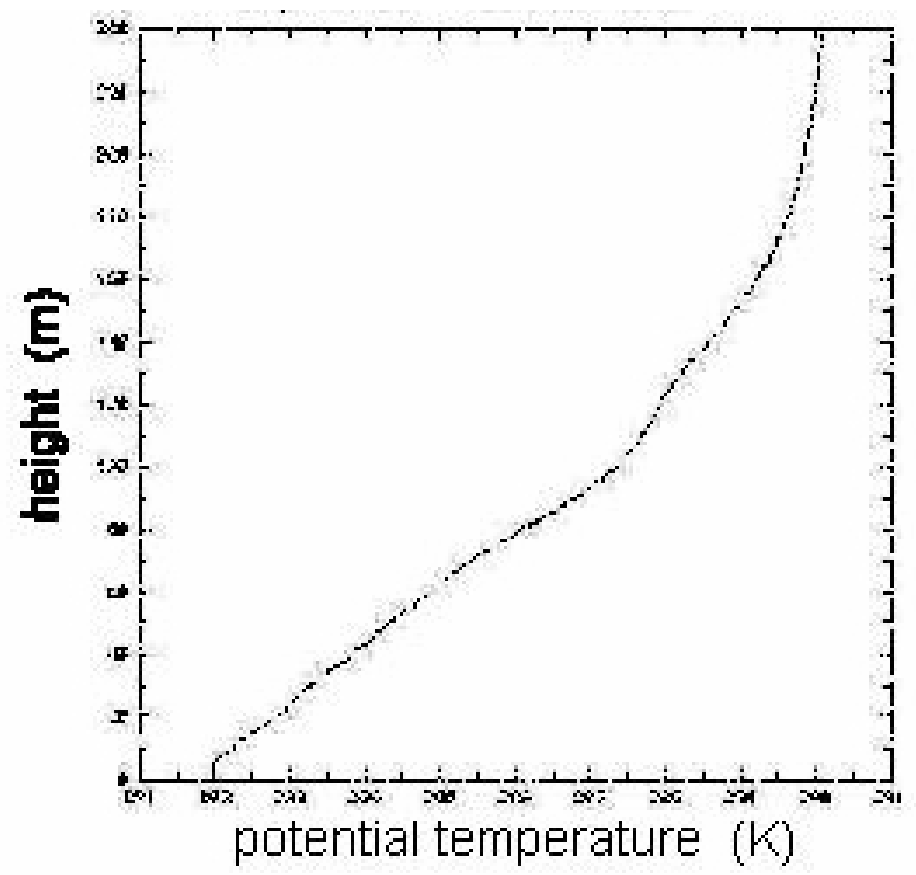

(a)

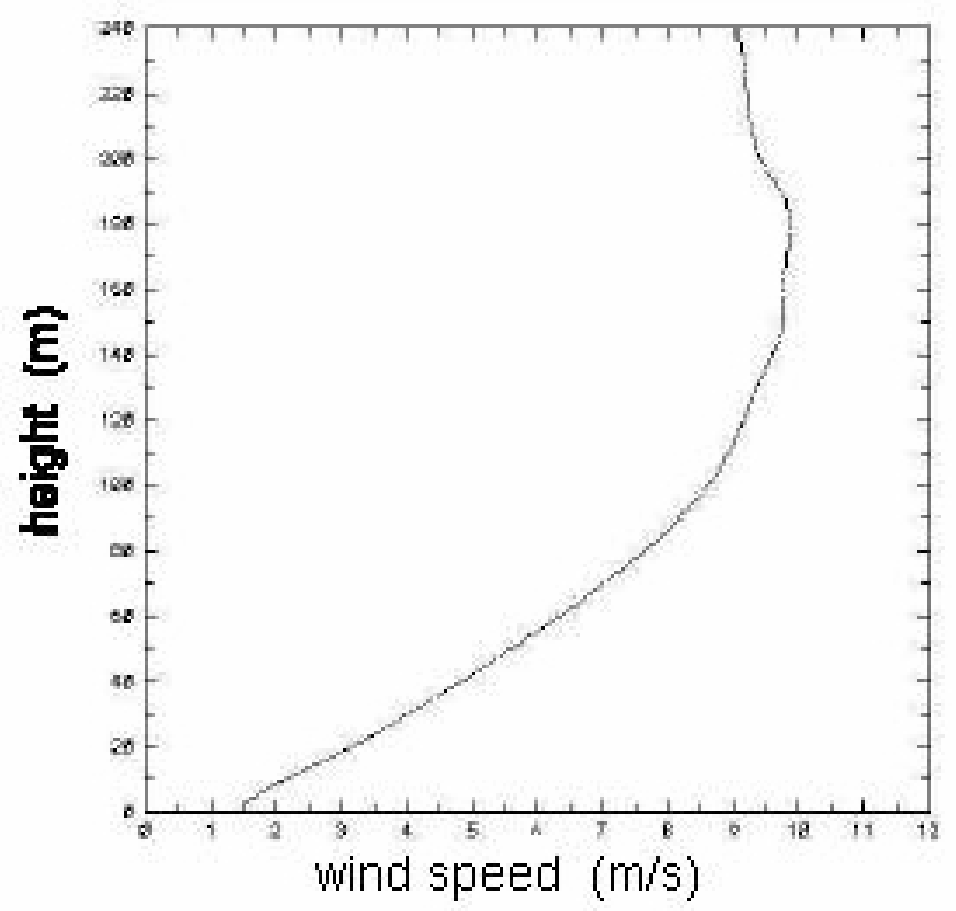

(b)

Figure 6.24: Vertical profiles $(0-240 \mathrm{~m})$ of simulated (a) potential temperature $(281$ $291 \mathrm{~K})$ and $(\mathrm{b})$ wind speed $(0-11 \mathrm{~m} / \mathrm{s})$ for Run $G 7.5 w t-02$ at hour 11.5 . 

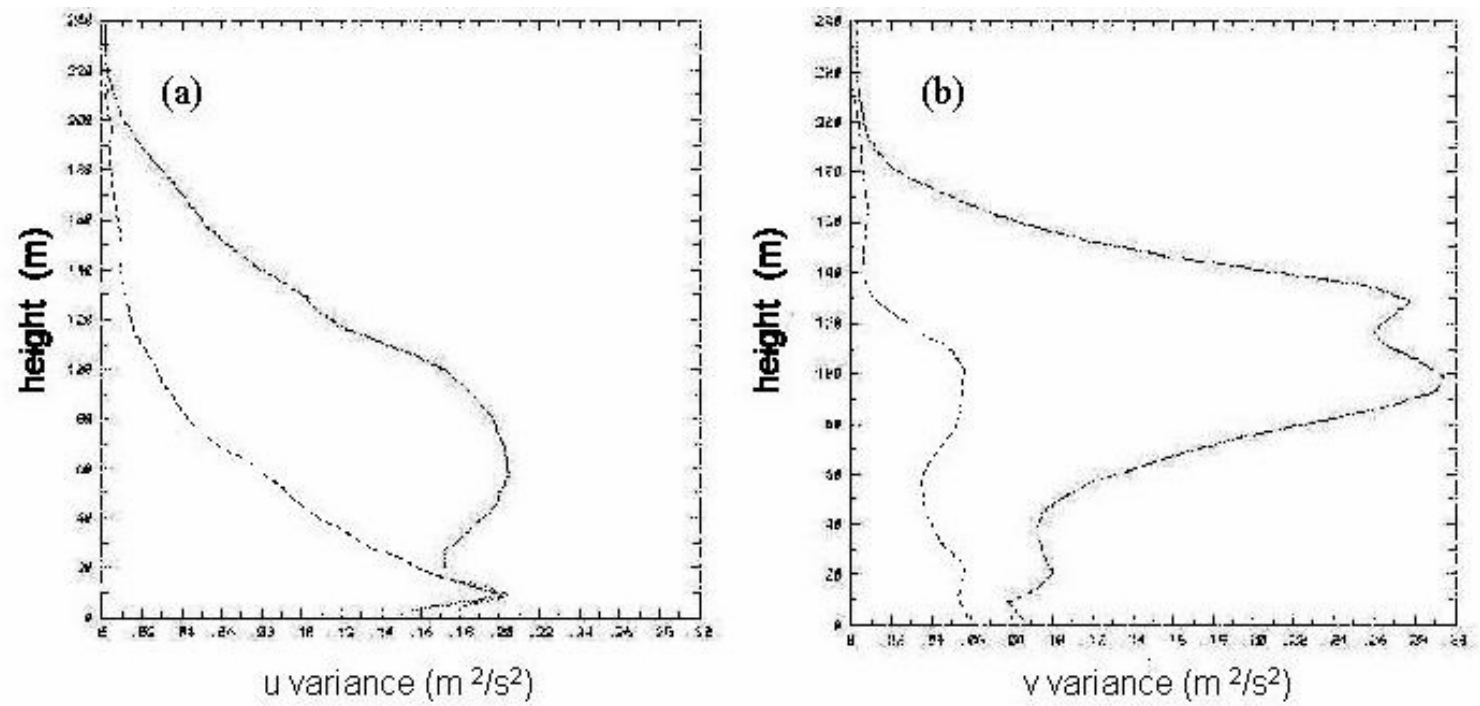

Figure 6.25: Vertical profiles of simulated (a) u velocity variance and (b) v velocity variance for Run G7.5wt-02 at hour 11.25 (dashed lines) and at hour 11.5 (solid lines); vertical axis is 0 to $240 \mathrm{~m}$ and horizontal axis is 0 to $0.3 \mathrm{~m}^{2} / \mathrm{s}^{2}$.
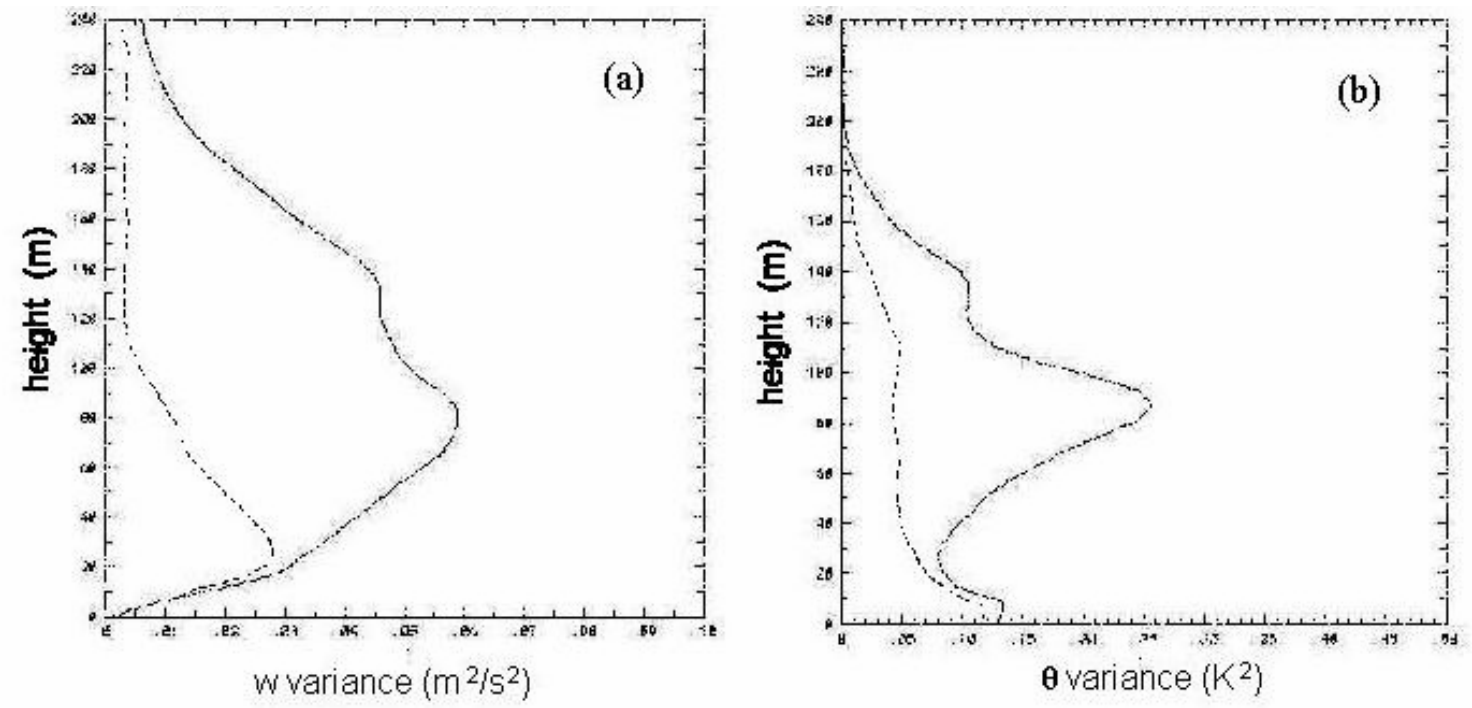

Figure 6.26: Vertical profiles of simulated (a) w velocity variance and (b) potential temperature variance for Run G7.5wt-02 at hour 11.25 (dashed lines) and at hour 11.5 (solid lines); vertical axis is 0 to $240 \mathrm{~m}$ and horizontal axis is 0 to $0.1 \mathrm{~m}^{2} / \mathrm{s}^{2}$ for (a), and 0 to 0.5 $K^{2}$ for (b). 

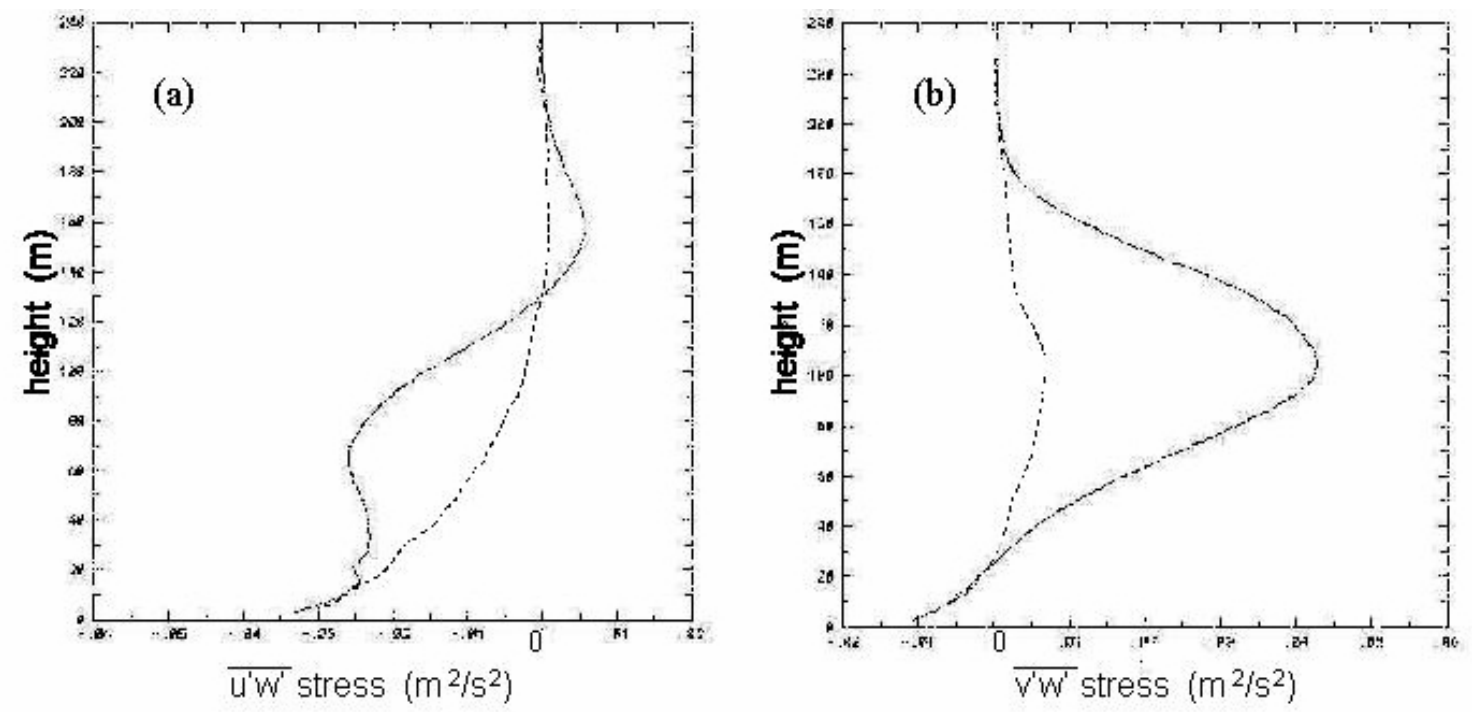

Figure 6.27: Vertical profiles of simulated (a) $\overline{u^{\prime} w^{\prime}}$ stress and (b) $\overline{v^{\prime} w^{\prime}}$ stress for Run G7.5wt-02 at hour 11.25 (dashed lines) and at hour 11.5 (solid lines); vertical axis is 0 to $240 \mathrm{~m}$ and horizontal axis is -0.6 to $0.2 \mathrm{~m}^{2} / \mathrm{s}^{2}$ for (a), and -0.2 to $0.6 \mathrm{~m}^{2} / \mathrm{s}^{2}$ for (b).
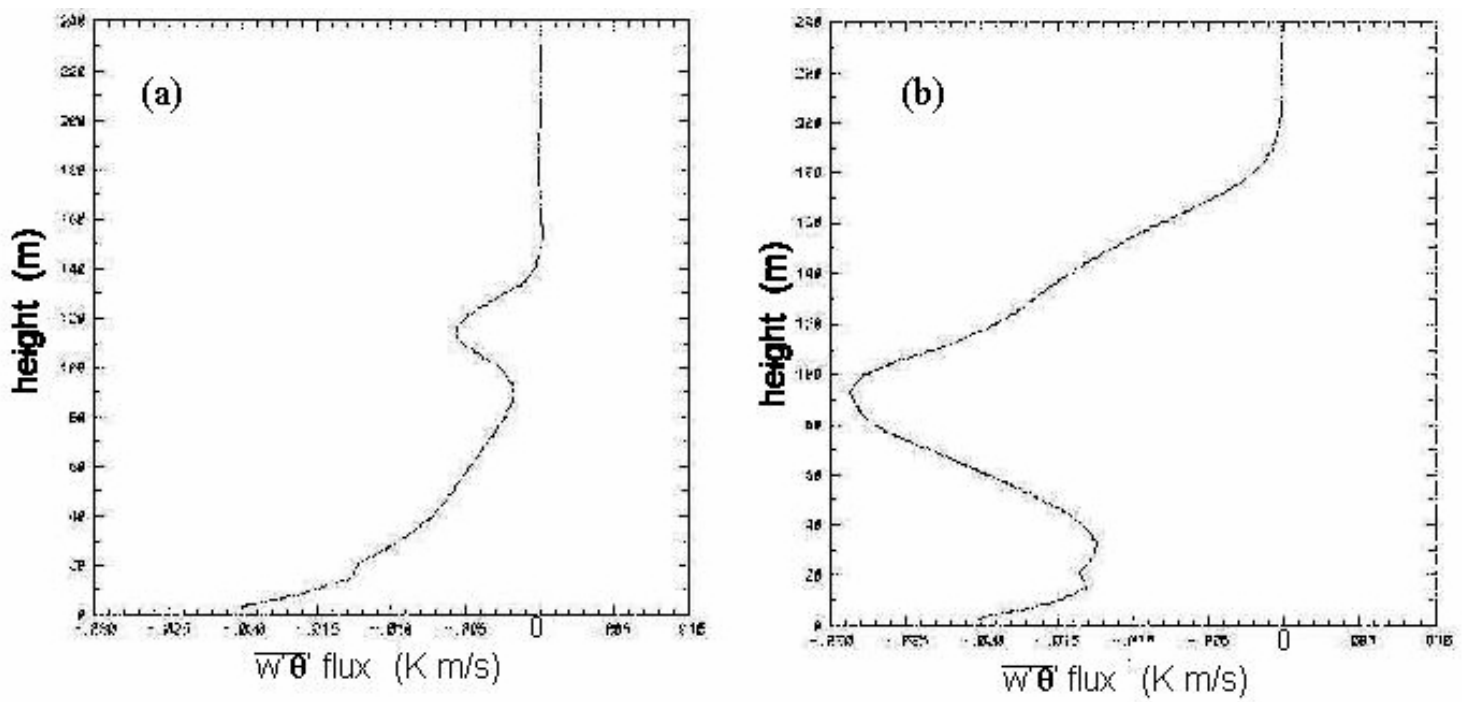

Figure 6.28: Vertical profiles of simulated heat flux for Run G7.5wt-02 at (a) hour 11.25 (onset of event) and (b) hour 11.5 (peak of event) ; vertical axis is 0 to $240 \mathrm{~m}$ and horizontal axis is -0.03 to $0.01 \mathrm{Km} / \mathrm{s}$. 

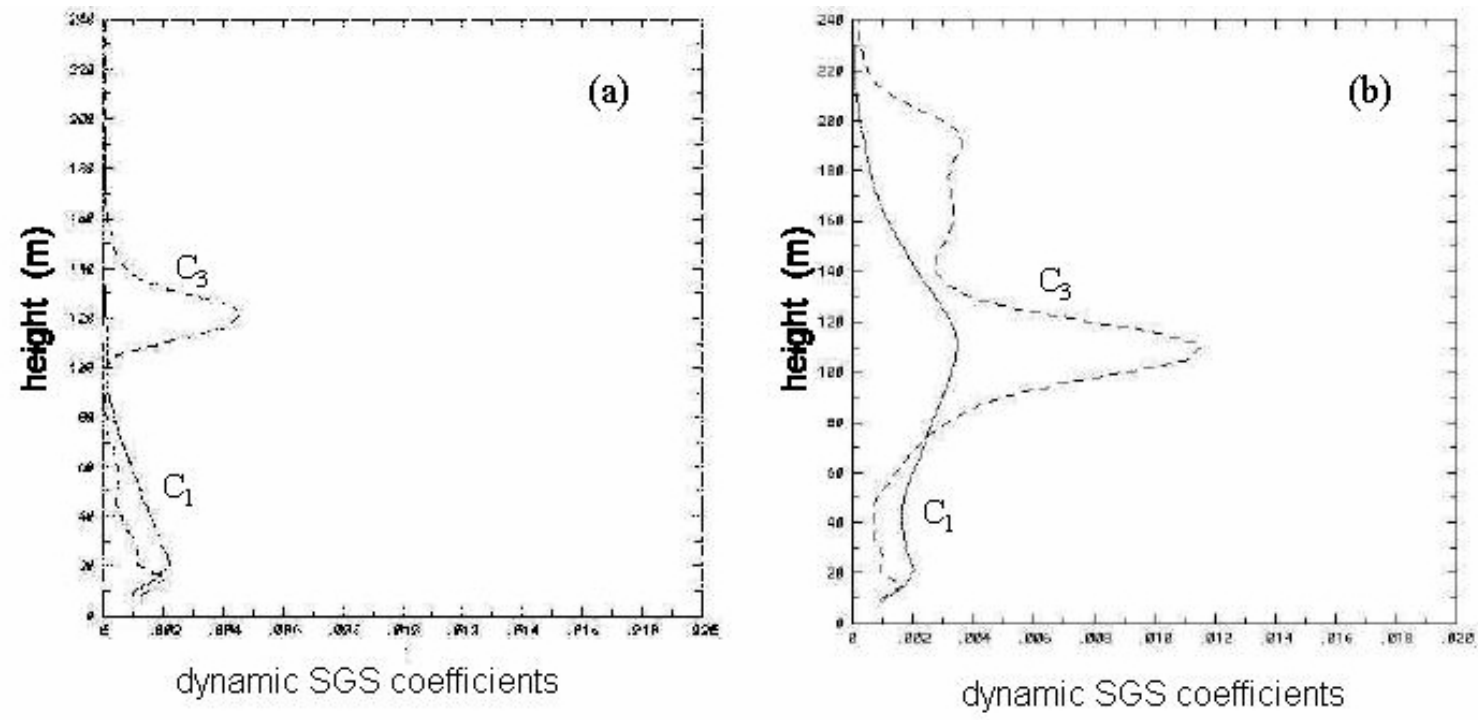

Figure 6.29: SGS model dynamic coefficients for Run $67.5 w t-02, C_{1}$ for SGS stress (solid lines) and $C_{3}$ for SGS heat flux (dashed lines), at (a) hour 11.25 (onset of event) and (b) hour 11.5 (peak of event) ; vertical axis is 0 to $240 \mathrm{~m}$ and horizontal axis is 0 to 0.02 . 

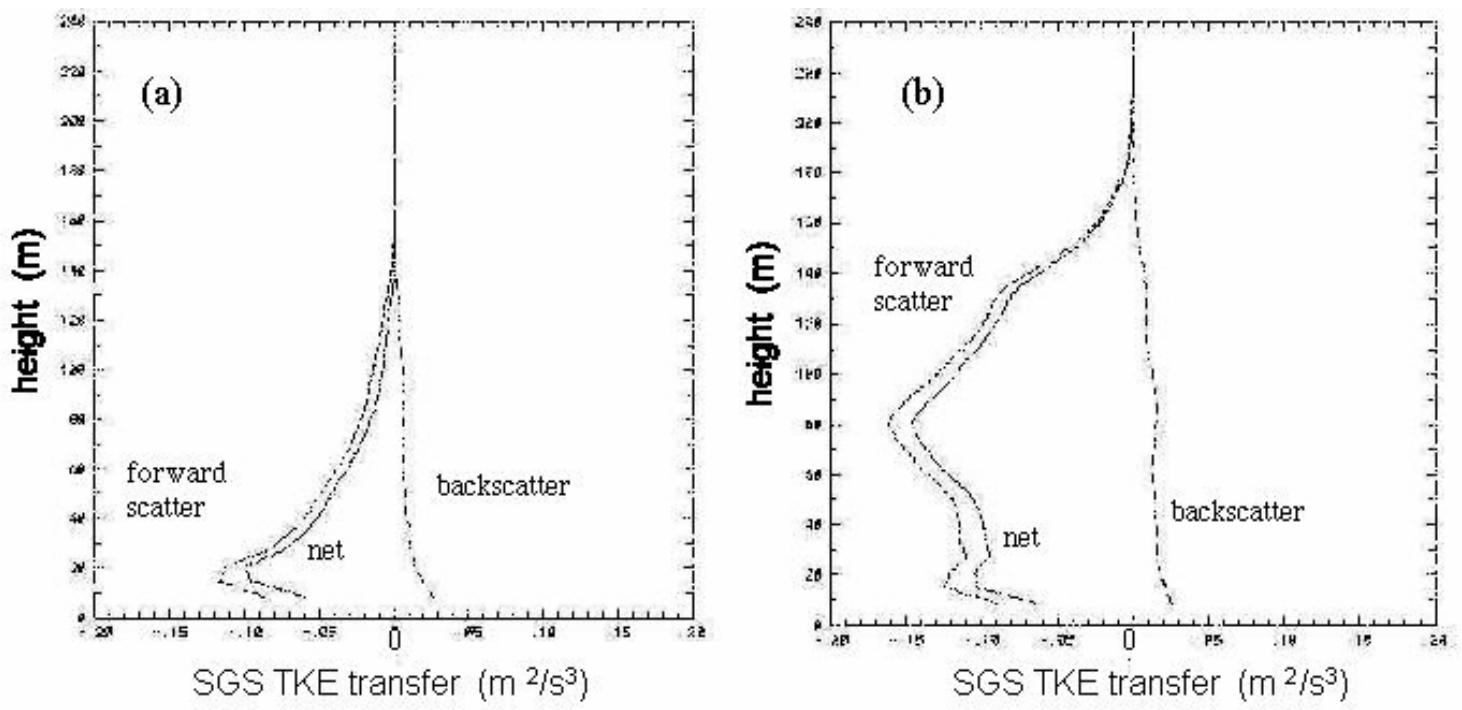

Figure 6.30: SGS TKE transfer for Run G7.5wt-02, indicating forward scatter, backscatter, and net transfer, at (a) hour 11.25 (onset of event) and (b) hour 11.5 (peak of event) ; vertical axis is 0 to $240 \mathrm{~m}$ and horizontal axis is -0.2 to $0.2 \times 10^{-2} \mathrm{~m}^{2} / \mathrm{s}^{3}$.
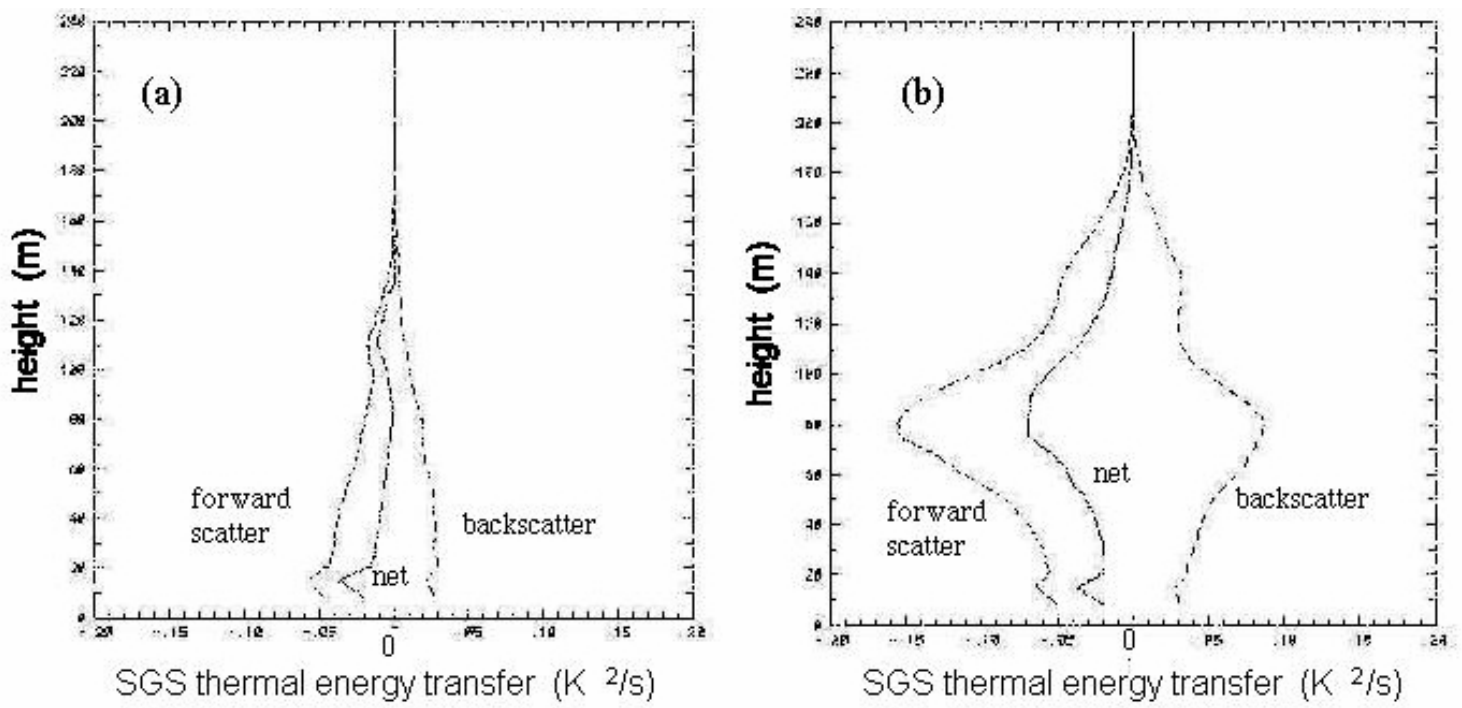

Figure 6.31: SGS thermal energy transfer for Run G7.5wt-02, indicating forward scatter, backscatter, and net transfer, at (a) hour 11.25 (onset of event) and (b) hour 11.5 (peak of event); vertical axis is 0 to $240 \mathrm{~m}$ and horizontal axis is -0.2 to $0.2 \times 10^{-2} \mathrm{~m}^{2} / \mathrm{s}^{3}$. 


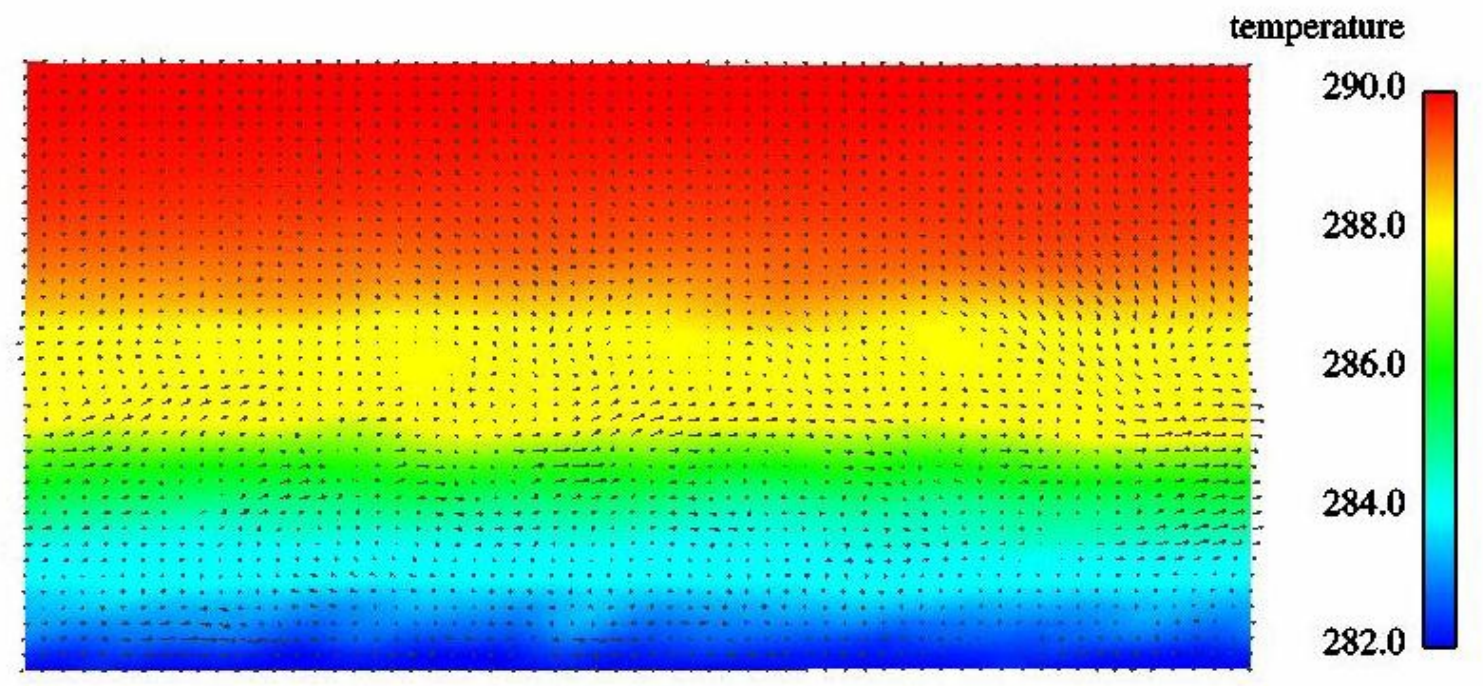

Figure 6.32: Temperature contours with fluctuating velocities for Run G7.5wt-02 near episode onset $(60 \mathrm{sec})$; vertical axis is height $(0-240 \mathrm{~m})$ and horizontal axis is the west-east direction $(0-960 \mathrm{~m})$; vertical scale stretched by factor of two, and fluctuating velocities scaled by factor of 20 for visualization.

temperature
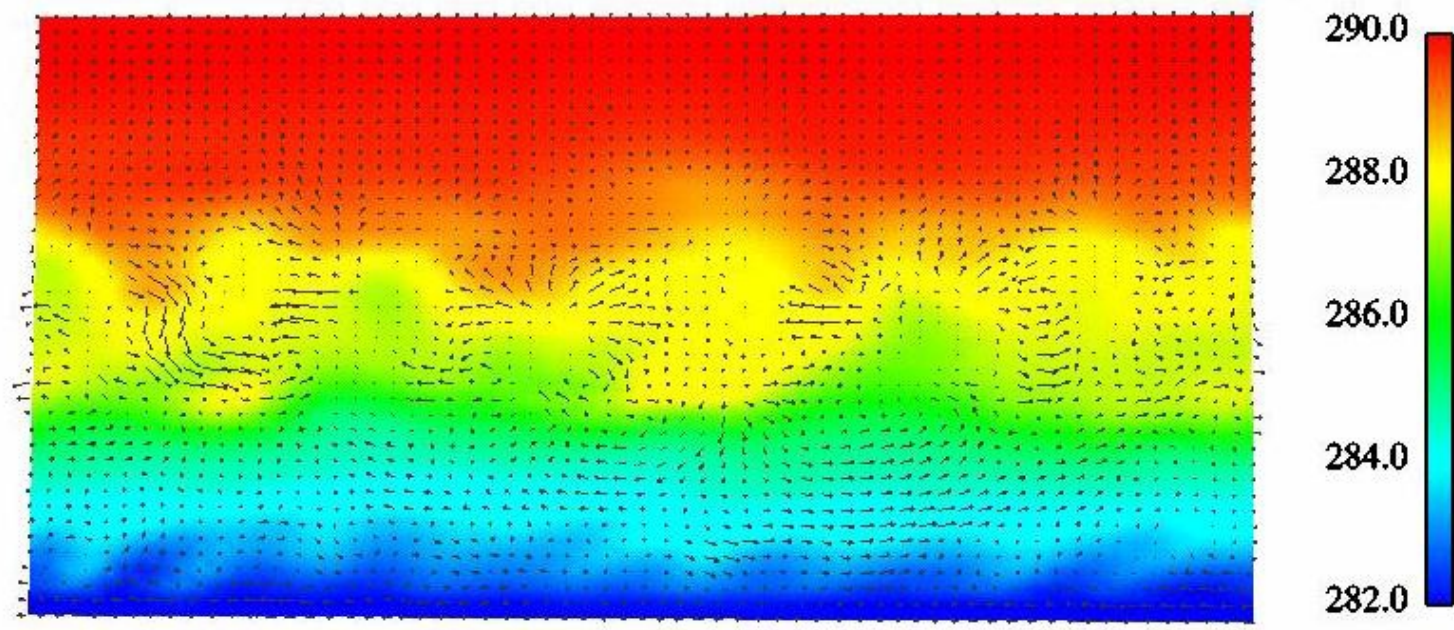

Figure 6.33: Temperature contours with fluctuating velocities for Run G7.5wt-02 during turbulence episode $(480 \mathrm{sec})$; vertical axis is height $(0-240 \mathrm{~m})$ and horizontal axis is the west-east direction $(0-960 \mathrm{~m})$; vertical scale stretched by factor of two, and fluctuating velocities scaled by factor of 20 for visualization. 
temperature

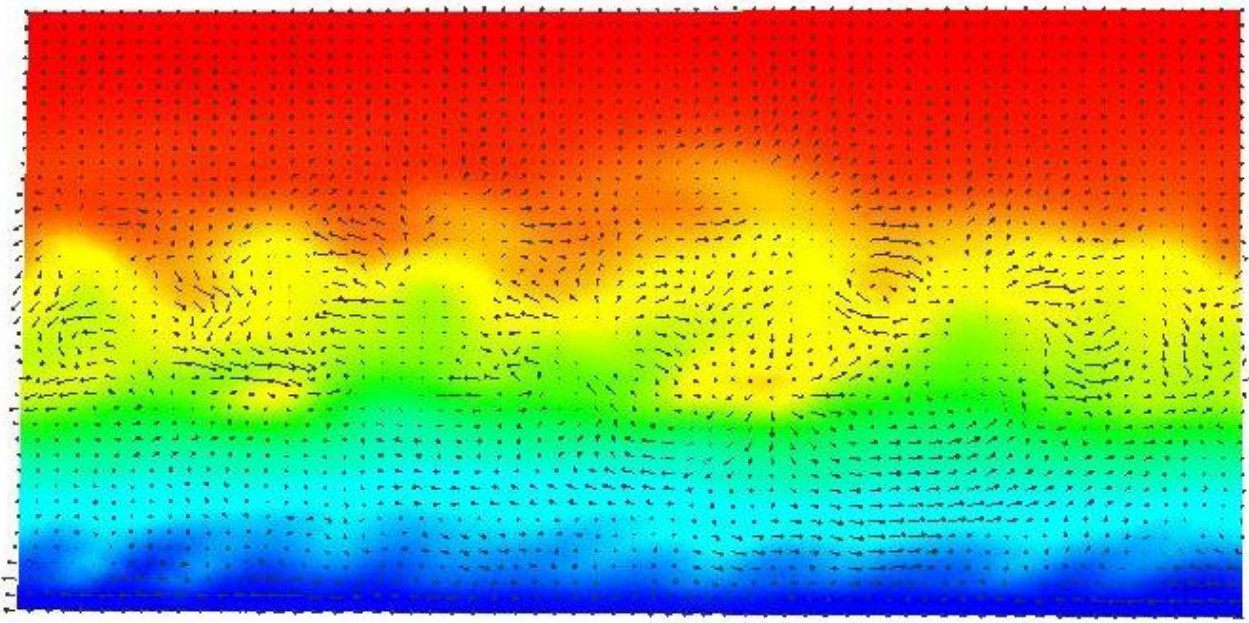

290.0

288.0

286.0

284.0

282.0

Figure 6.34: Temperature contours with fluctuating velocities for Run G\%.5wt-02 during turbulence episode $(486 \mathrm{sec})$; vertical axis is height $(0-240 \mathrm{~m})$ and horizontal axis is the west-east direction $(0-960 \mathrm{~m})$; vertical scale stretched by factor of two, and fluctuating velocities scaled by factor of 20 for visualization.

temperature
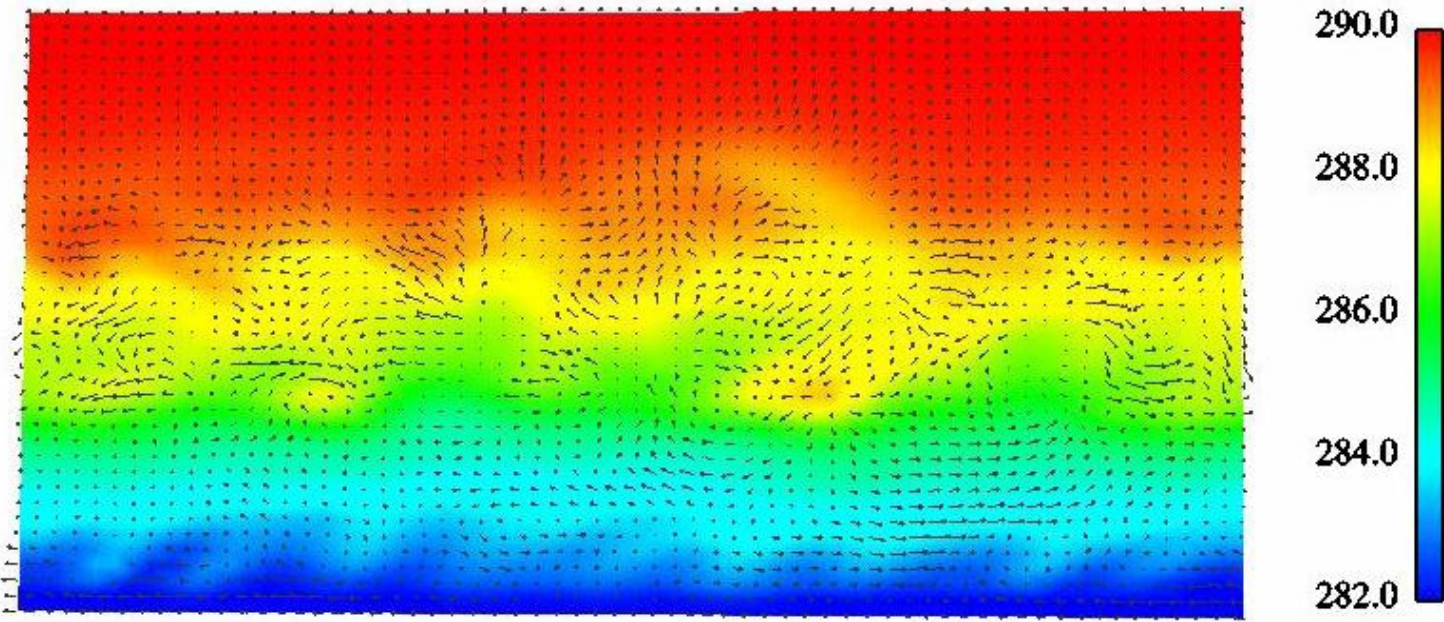

Figure 6.35: Temperature contours with fluctuating velocities for Run G7.5wt-02 during turbulence episode $(492 \mathrm{sec})$; vertical axis is height $(0-240 \mathrm{~m})$ and horizontal axis is the west-east direction $(0-960 \mathrm{~m})$; vertical scale stretched by factor of two, and fluctuating velocities scaled by factor of 20 for visualization. 


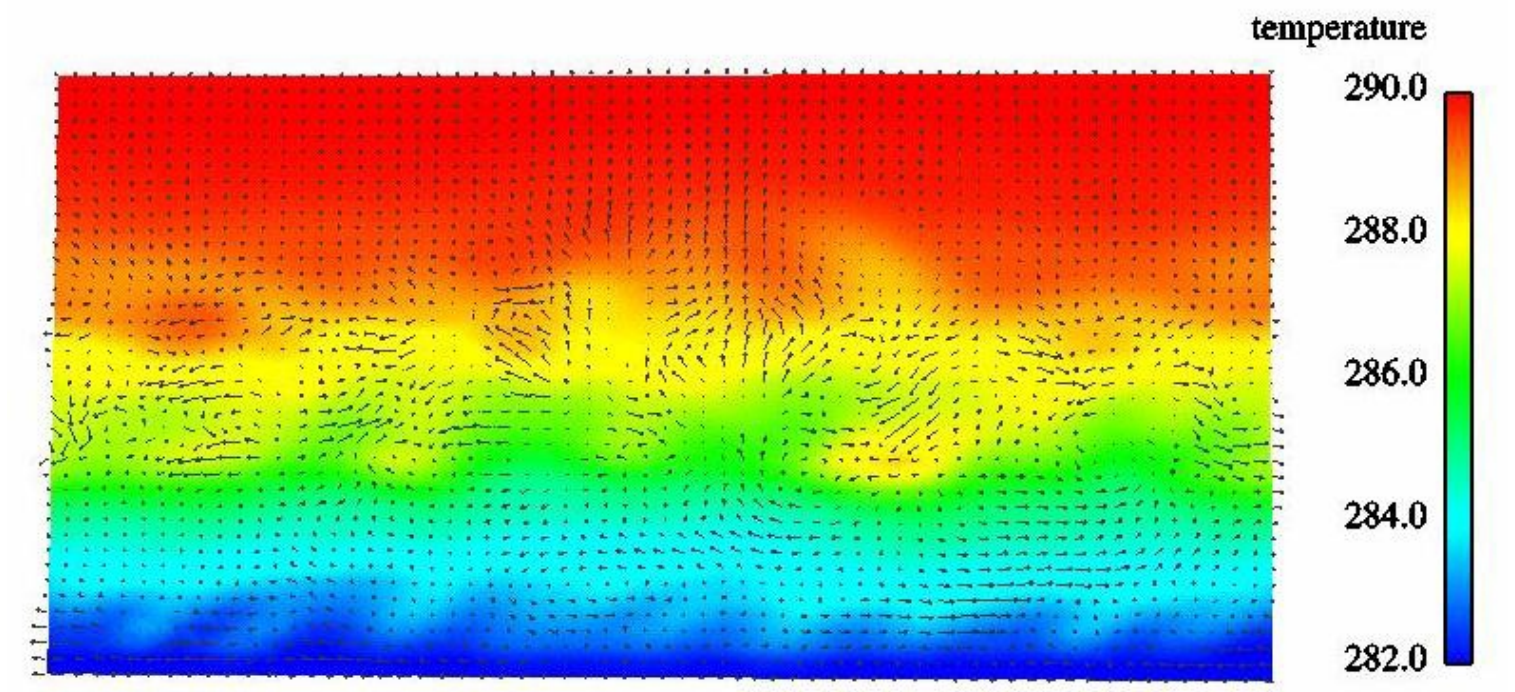

Figure 6.36: Temperature contours with fluctuating velocities for Run G7.5wt-02 during turbulence episode $(498 \mathrm{sec})$; vertical axis is height $(0-240 \mathrm{~m})$ and horizontal axis is the west-east direction $(0-960 \mathrm{~m})$; vertical scale stretched by factor of two, and fluctuating velocities scaled by factor of 20 for visualization.

temperature
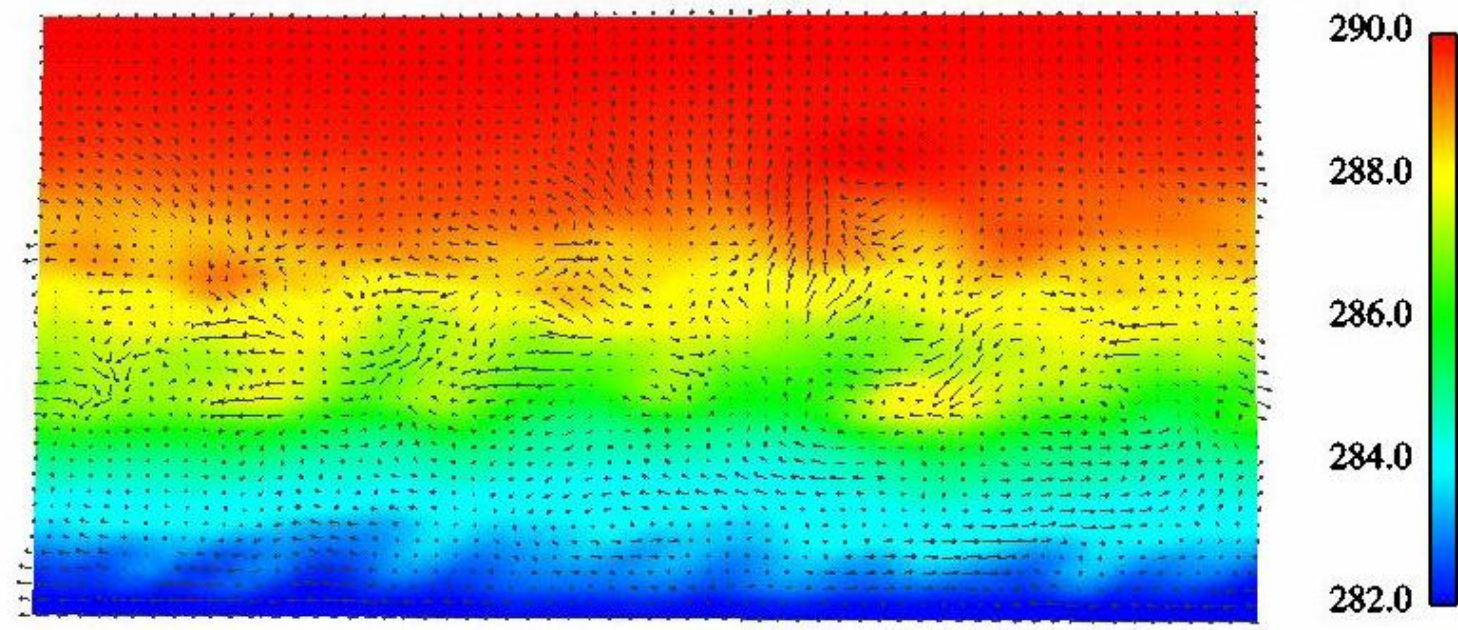

Figure 6.37: Temperature contours with fluctuating velocities for Run G7.5wt-02 during turbulence episode $(504 \mathrm{sec})$; vertical axis is height $(0-240 \mathrm{~m})$ and horizontal axis is the west-east direction $(0-960 \mathrm{~m})$; vertical scale stretched by factor of two, and fluctuating velocities scaled by factor of 20 for visualization. 


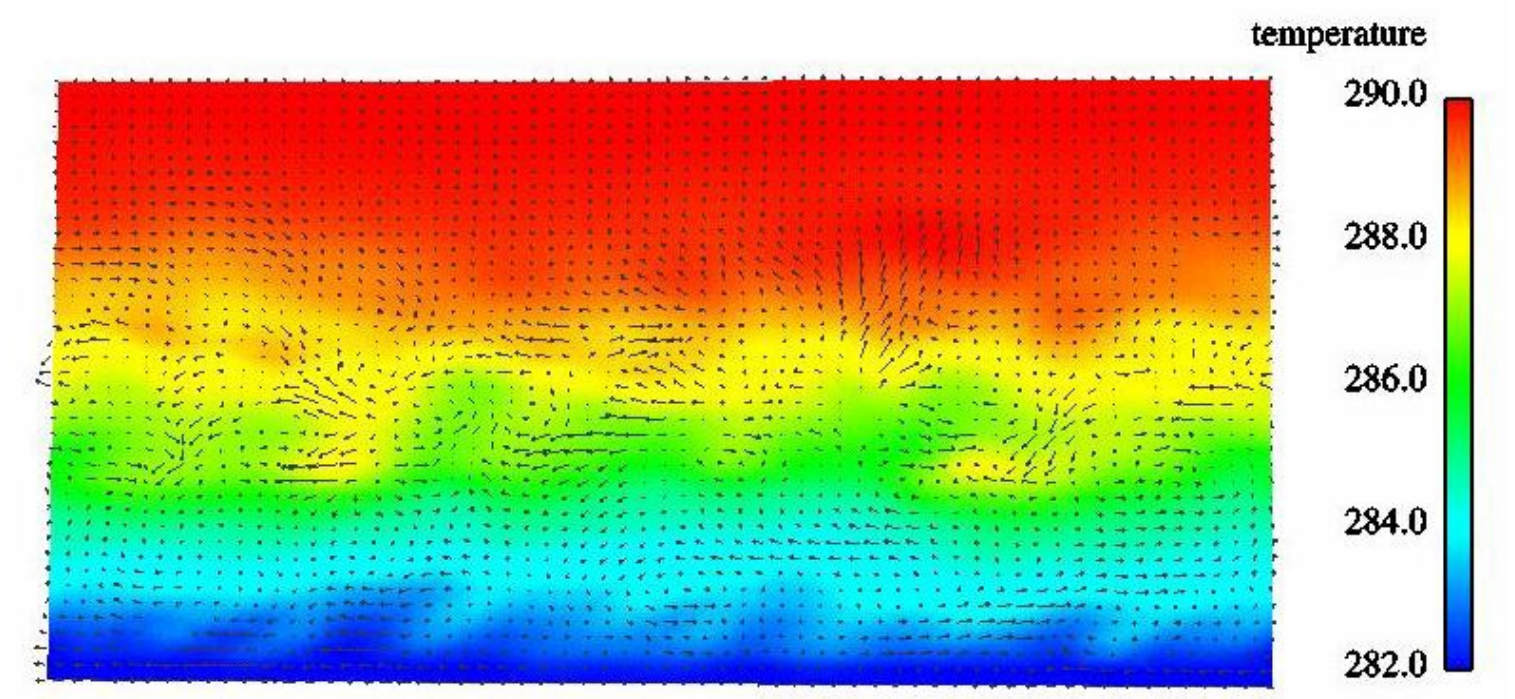

Figure 6.38: Temperature contours with fluctuating velocities for Run G7.5wt-02 during turbulence episode $(510 \mathrm{sec})$; vertical axis is height $(0-240 \mathrm{~m})$ and horizontal axis is the west-east direction $(0-960 \mathrm{~m})$; vertical scale stretched by factor of two, and fluctuating velocities scaled by factor of 20 for visualization.

temperature
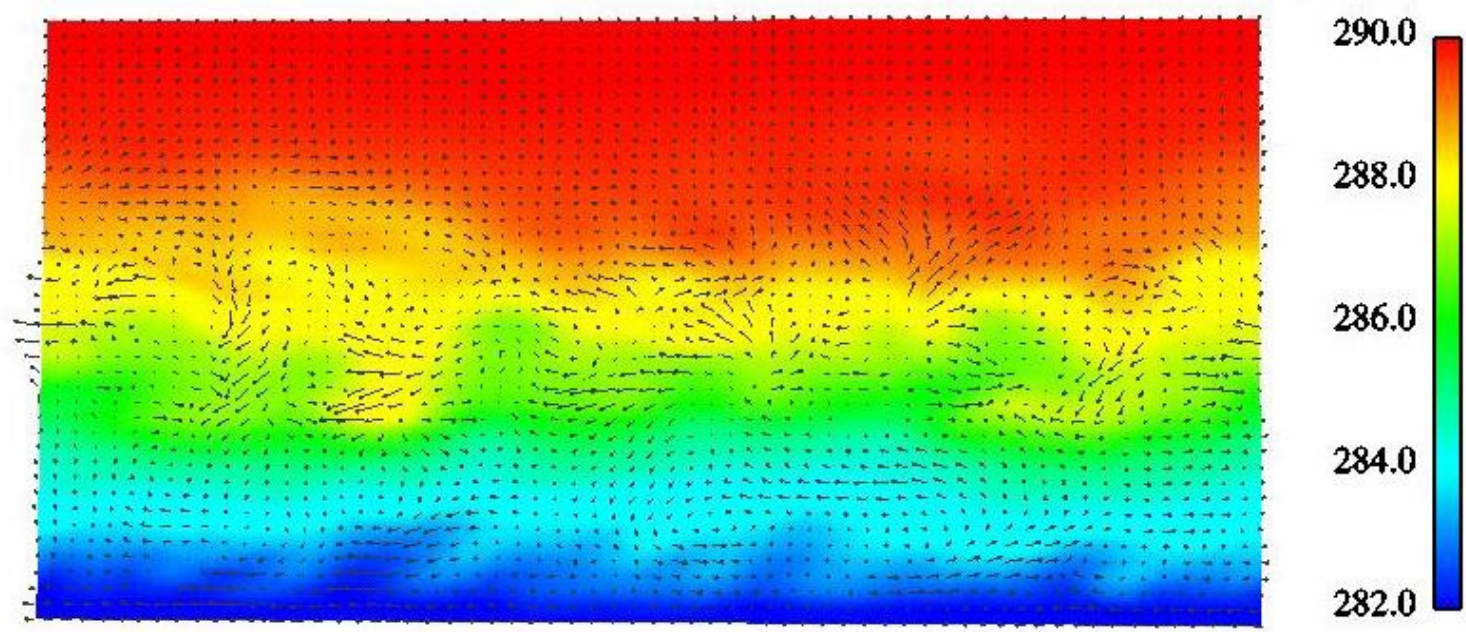

Figure 6.39: Temperature contours with fluctuating velocities for Run G7.5wt-02 during turbulence episode $(516 \mathrm{sec})$; vertical axis is height $(0-240 \mathrm{~m})$ and horizontal axis is the west-east direction $(0-960 \mathrm{~m})$; vertical scale stretched by factor of two, and fluctuating velocities scaled by factor of 20 for visualization. 


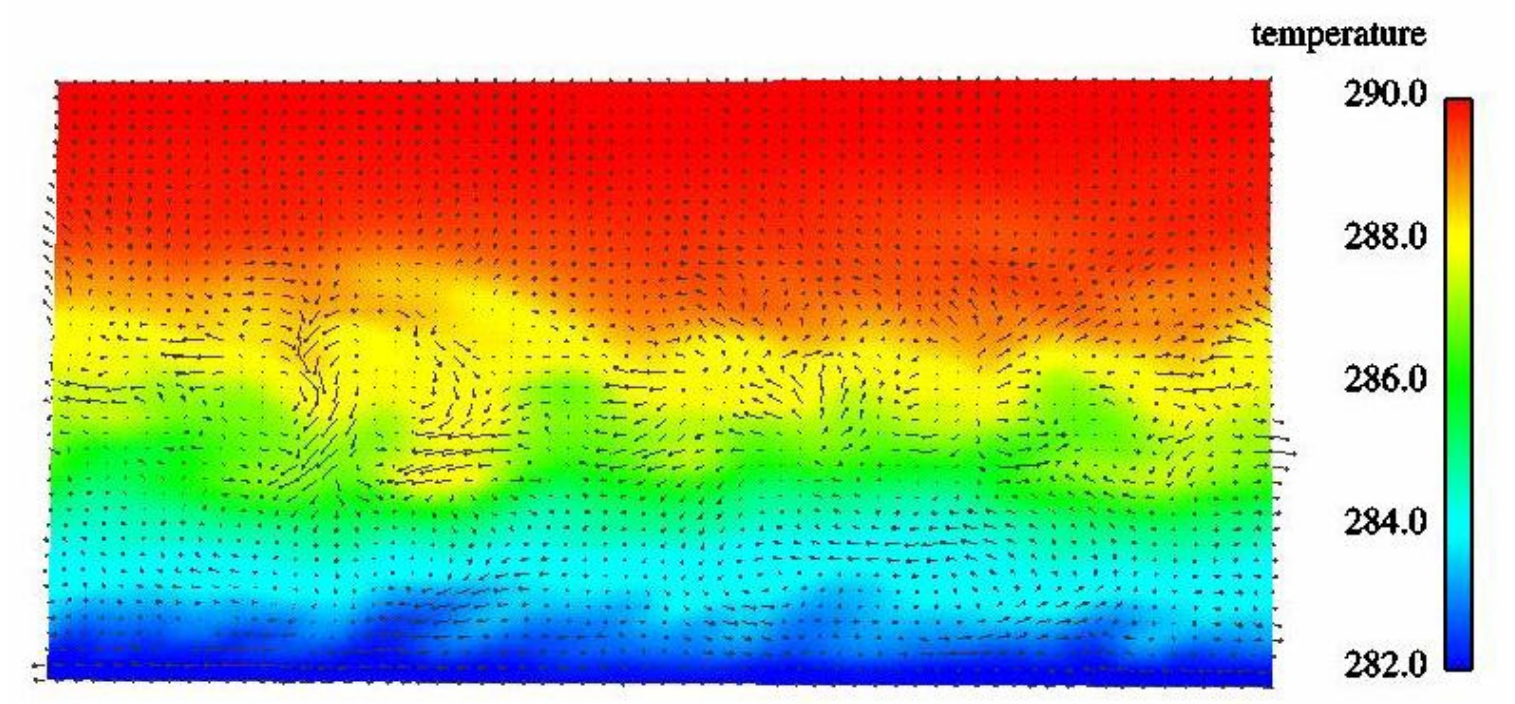

Figure 6.40: Temperature contours with fluctuating velocities for Run G7.5wt-02 during turbulence episode $(522 \mathrm{sec})$; vertical axis is height $(0-240 \mathrm{~m})$ and horizontal axis is the west-east direction $(0-960 \mathrm{~m})$; vertical scale stretched by factor of two, and fluctuating velocities scaled by factor of 20 for visualization.

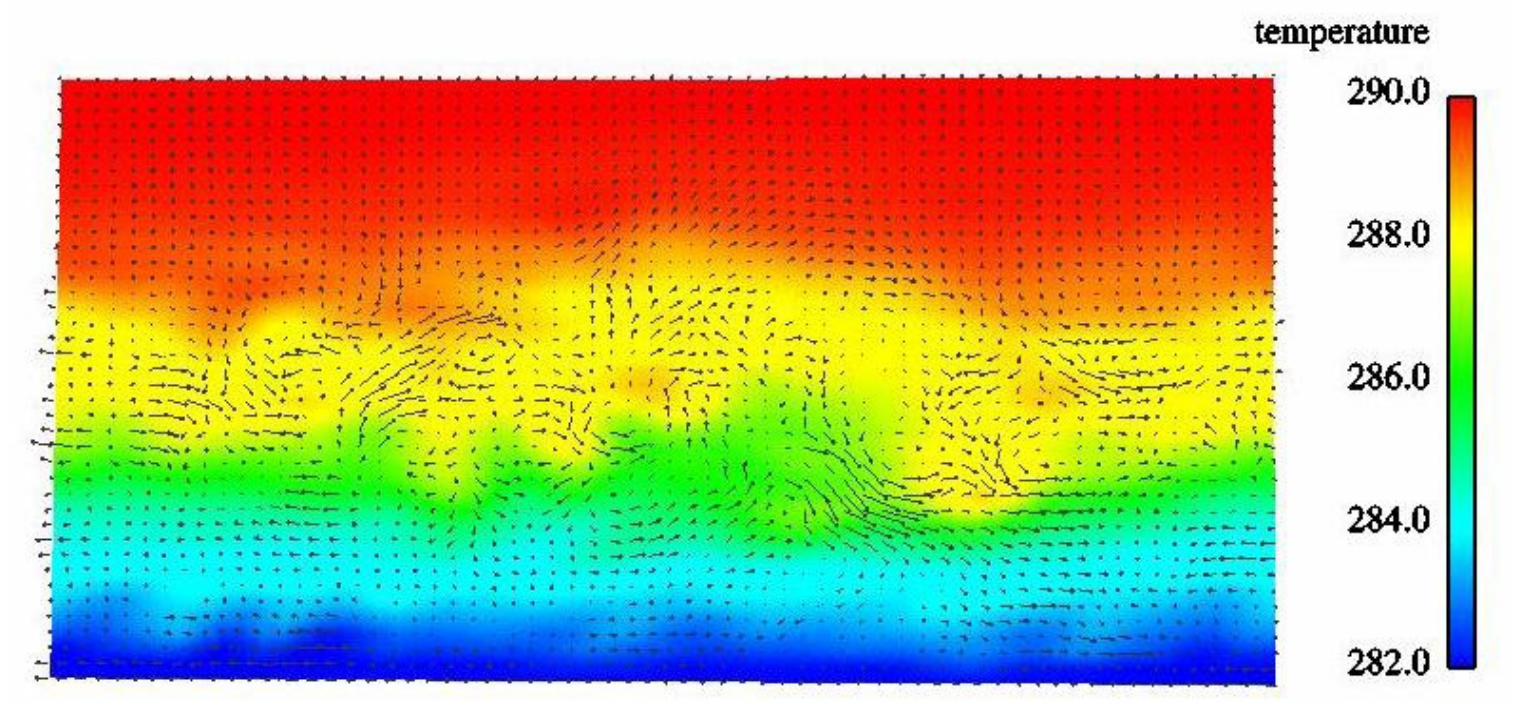

Figure 6.41: Temperature contours with fluctuating velocities for Run G7.5wt-02 approaching peak of episode $(570 \mathrm{sec})$; vertical axis is height $(0-240 \mathrm{~m})$ and horizontal axis is the west-east direction $(0-960 \mathrm{~m})$; vertical scale stretched by factor of two, and fluctuating velocities scaled by factor of 20 for visualization. 


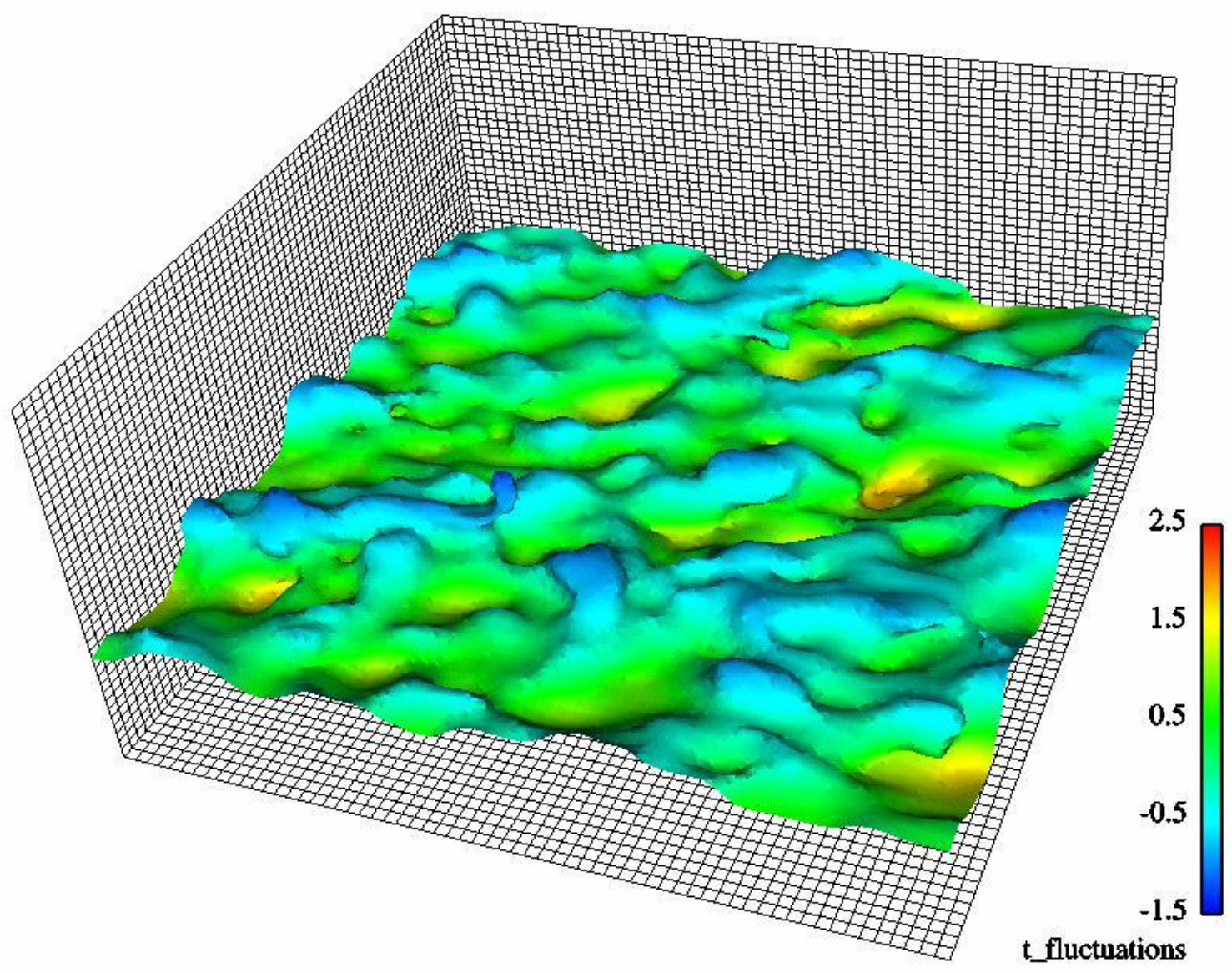

Figure 6.42: Potential temperature isosurface $(286.5 \mathrm{~K})$ colored by temperature fluctuations showing wave-like motions for Run $G 7.5 w t$-02 approaching peak of episode (570 sec); vertical axis is height $(0-240 \mathrm{~m})$ and the horizontal axes are $(0-960 \mathrm{~m})$ with the $\mathrm{x}$-axis (west-east) from left to right along the grid lines and the y-axis (south-north) from bottom to top along the grid lines; vertical scale stretched by factor of two for visualization. 


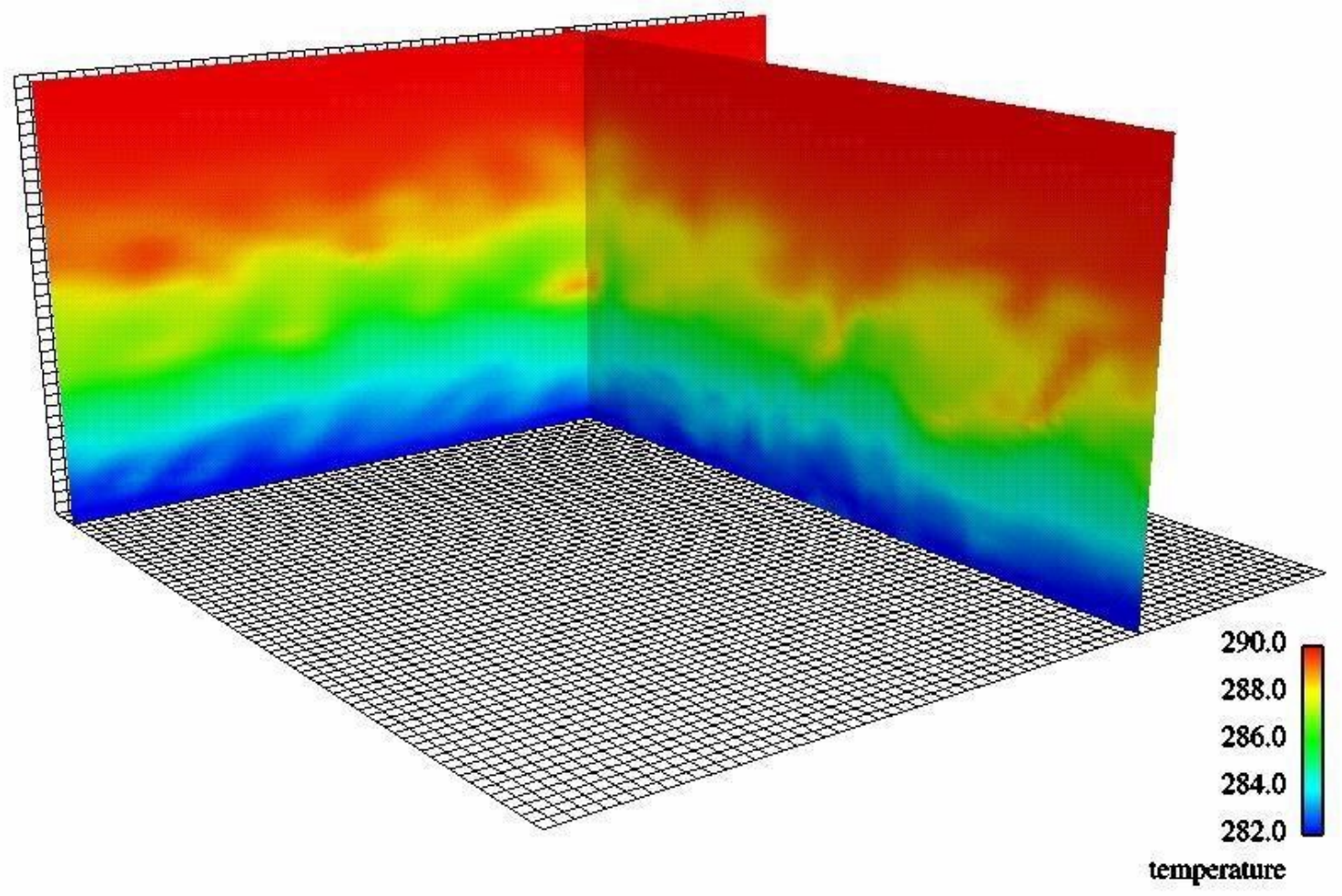

Figure 6.43: Temperature contours in the $\mathrm{x}-\mathrm{z}$ plane at $\mathrm{y}=900 \mathrm{~m}$ and in $\mathrm{y}-\mathrm{z}$ plane at $\mathrm{x}=$ $670 \mathrm{~m}$ for Run G7.5wt-02 during turbulence episode (492 sec); vertical axis is height (0 - 240 $\mathrm{m}$ ) and horizontal axis (lower center to upper right) is x-axis $(0-960 \mathrm{~m})$ and (lower center to upper left) is y-axis $(0-960 \mathrm{~m})$; vertical scale stretched by factor of two for visualization. 


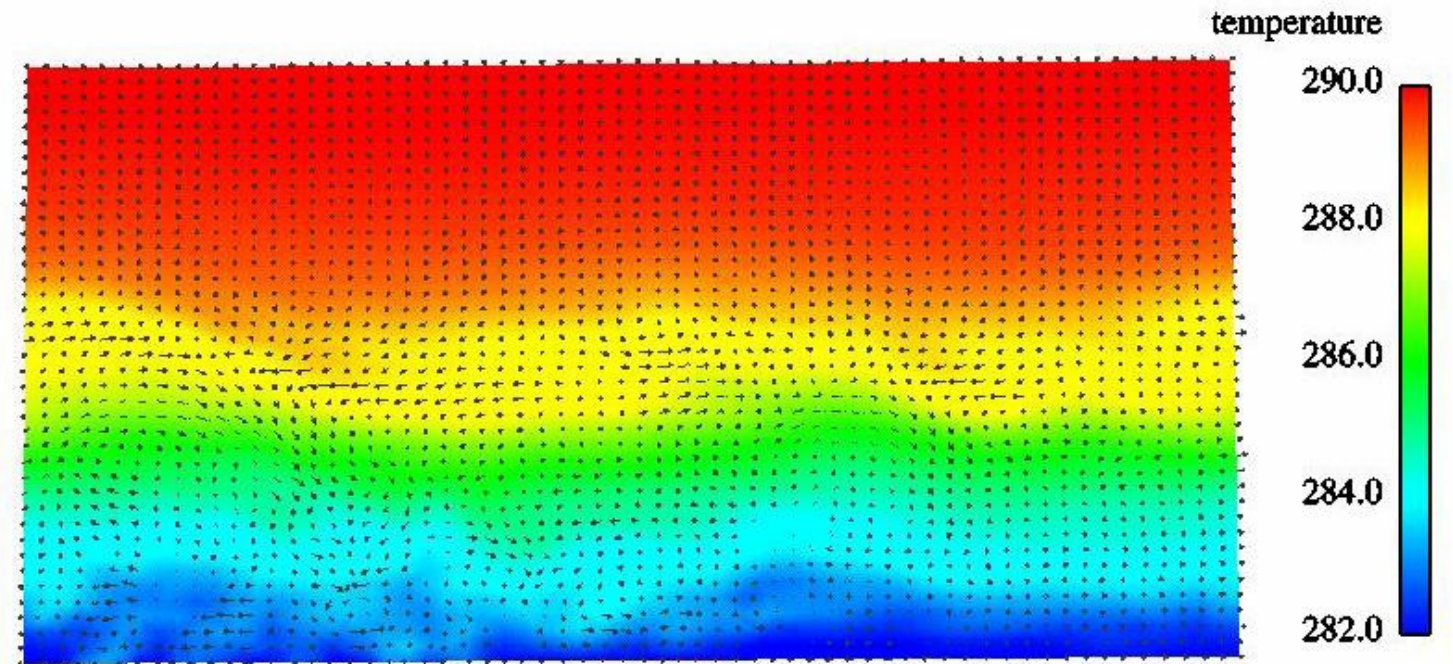

Figure 6.44: Temperature contours with fluctuating velocities in $\mathrm{y}-\mathrm{z}$ plane at $\mathrm{x}=670 \mathrm{~m}$ for Run G7.5wt-02 near turbulence episode onset $(60 \mathrm{sec})$; vertical axis is height $(0-240$ $\mathrm{m}$ ) and horizontal axis is $\mathrm{y}$-axis (0 - $960 \mathrm{~m}$ from left to right); vertical scale stretched by factor of two, and fluctuating velocities scaled by factor of 20 for visualization.

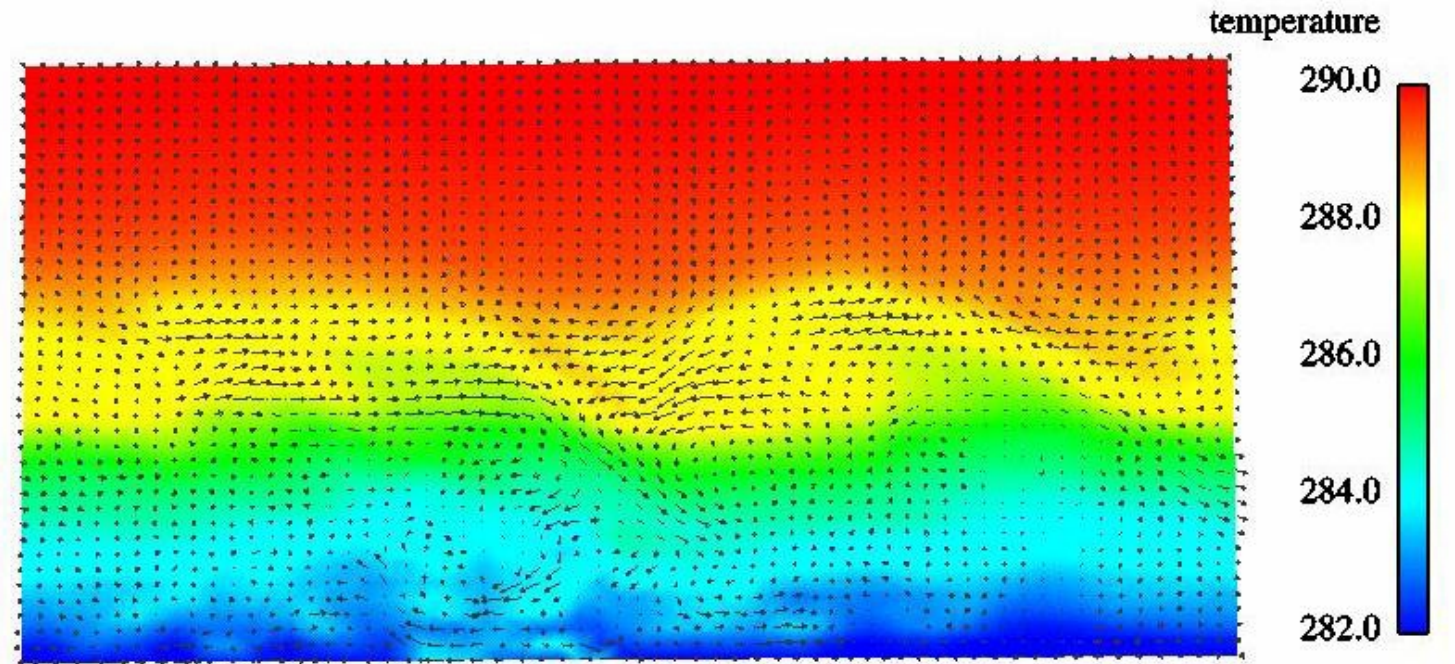

Figure 6.45: Temperature contours with fluctuating velocities in $\mathrm{y}-\mathrm{z}$ plane at $\mathrm{x}=670 \mathrm{~m}$ for Run G7.5wt-02 during turbulence episode (180 sec); vertical axis is height $(0-240 \mathrm{~m})$ and horizontal axis is y-axis (0 - $960 \mathrm{~m}$ from left to right); vertical scale stretched by factor of two, and fluctuating velocities scaled by factor of 20 for visualization. 


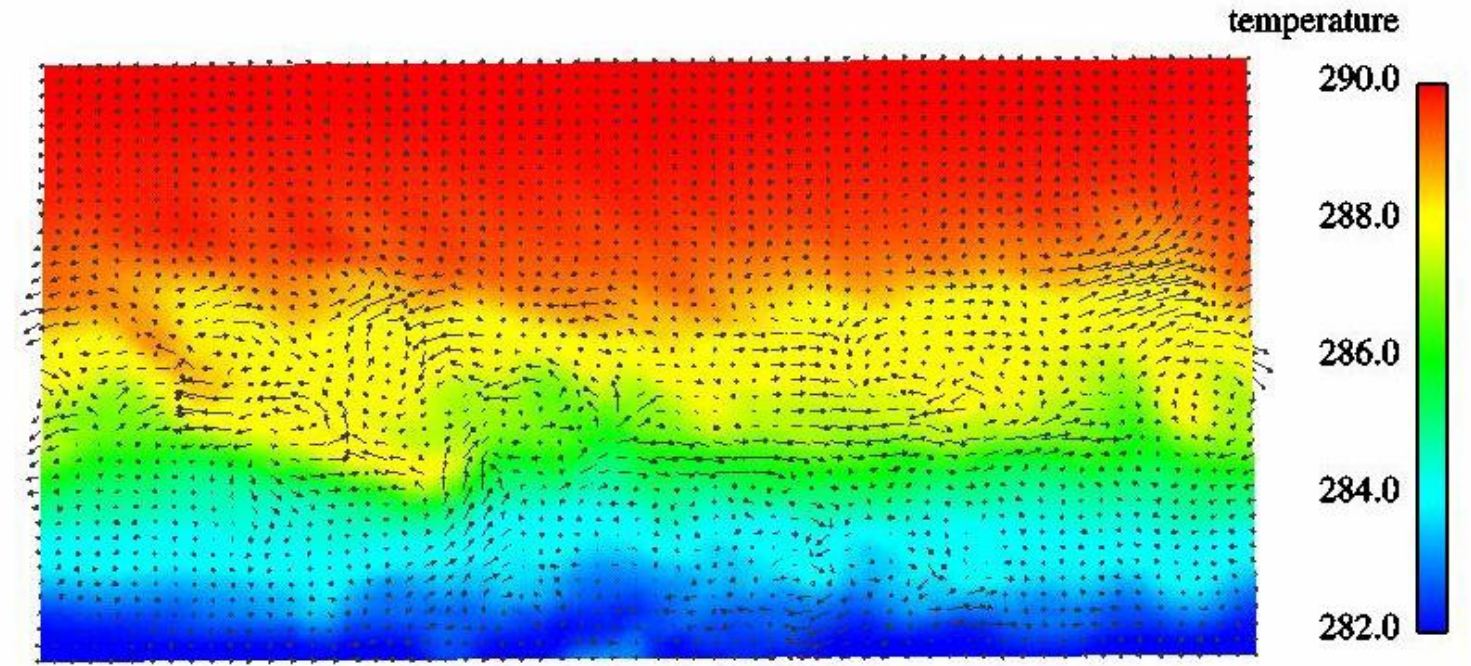

Figure 6.46: Temperature contours with fluctuating velocities in $\mathrm{y}$-z plane at $\mathrm{x}=670 \mathrm{~m}$ for Run G7.5wt-02 during turbulence episode (492 sec); vertical axis is height $(0-240 \mathrm{~m})$ and horizontal axis is y-axis (0 - $960 \mathrm{~m}$ from left to right); vertical scale stretched by factor of two, and fluctuating velocities scaled by factor of 20 for visualization.

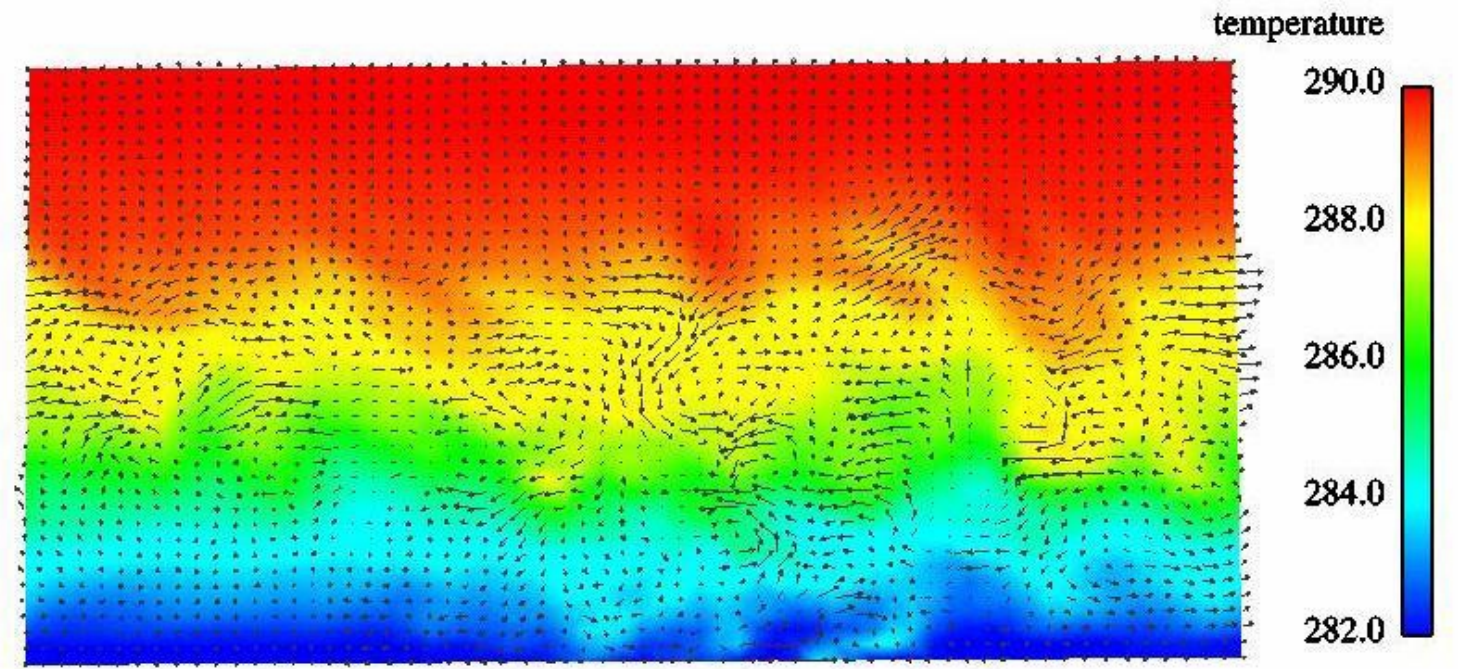

Figure 6.47: Temperature contours with fluctuating velocities in $\mathrm{y}-\mathrm{z}$ plane at $\mathrm{x}=670 \mathrm{~m}$ for Run G7.5wt-02 during turbulence episode (570 sec); vertical axis is height $(0-240 \mathrm{~m})$ and horizontal axis is y-axis (0 - $960 \mathrm{~m}$ from left to right); vertical scale stretched by factor of two, and fluctuating velocities scaled by factor of 20 for visualization. 


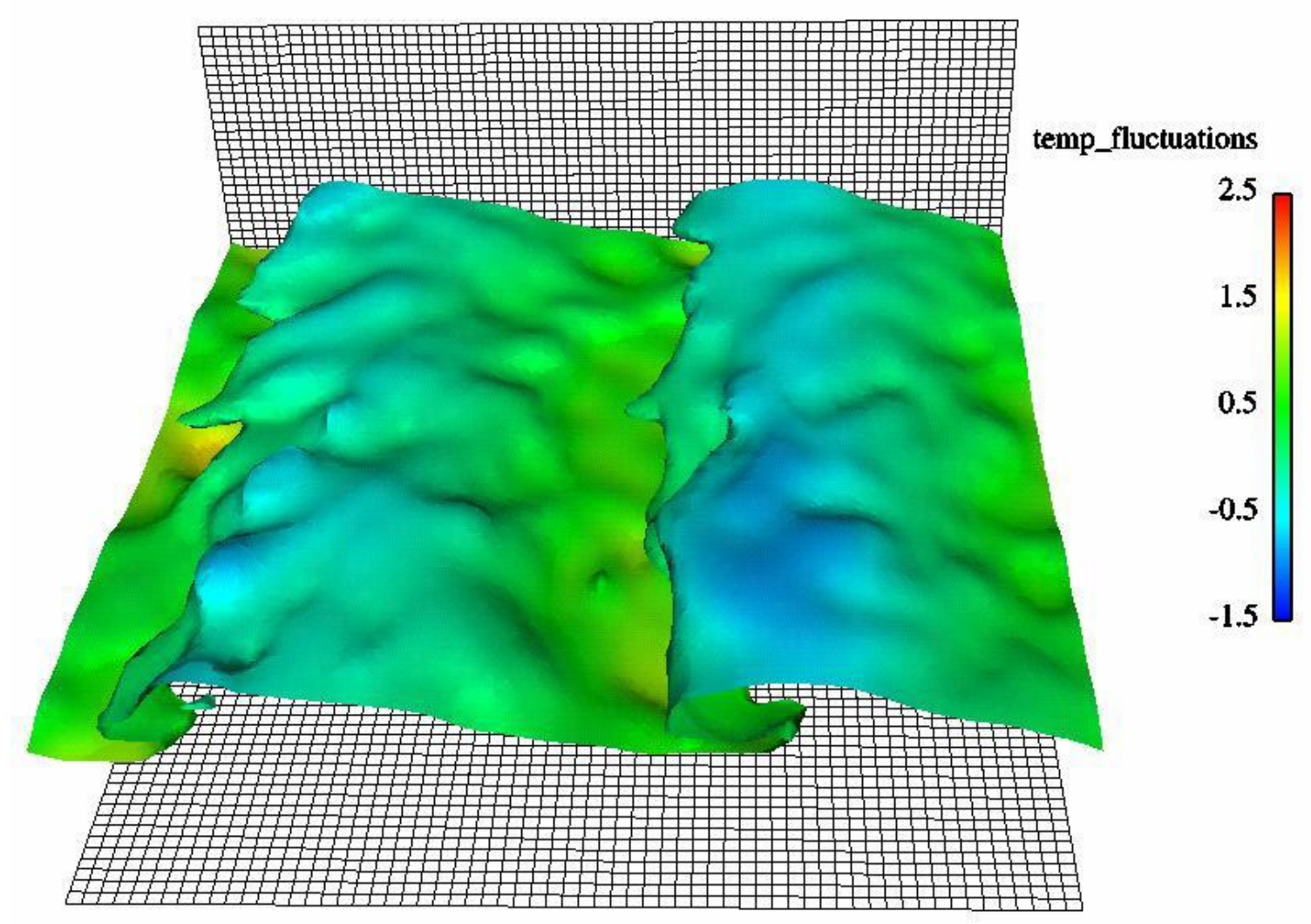

Figure 6.48: Potential temperature isosurface (288K) colored by temperature fluctuations showing large wave-like motions for Run G7.5wt-02 early in episode (180 sec); vertical axis is height $(0-240 \mathrm{~m})$ and horizontal axes are $\mathrm{y}$-axis $(0-960 \mathrm{~m}$ left to right) and $\mathrm{x}$-axis $(0$ - $960 \mathrm{~m}$ top to bottom), with $\mathrm{y}-\mathrm{z}$ reference plane at $\mathrm{x}=0 \mathrm{~m}$; vertical scale stretched by factor of two for visualization; the flow direction is along the $\mathrm{x}$-axis (top to bottom). 


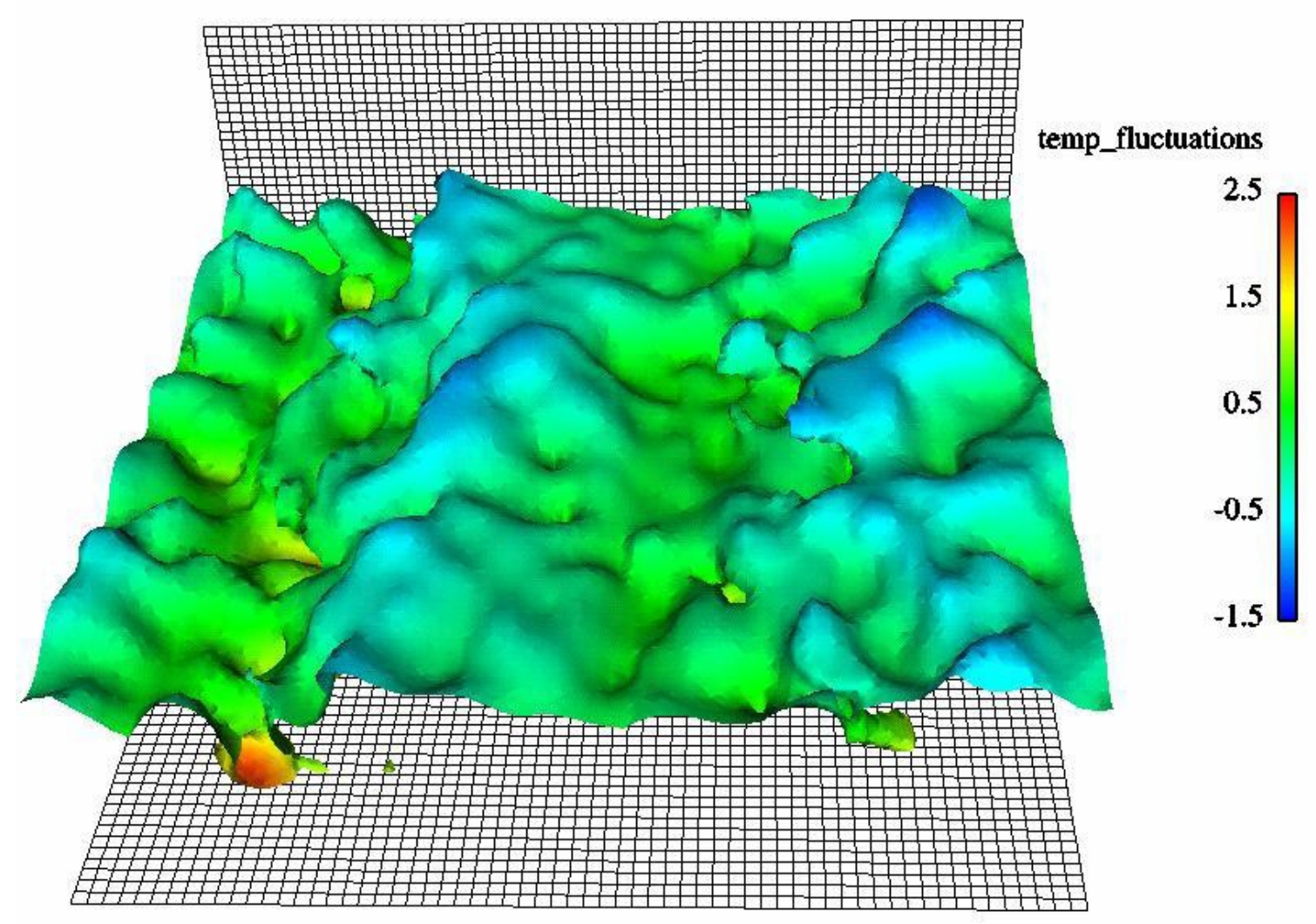

Figure 6.49: Potential temperature isosurface (288K) colored by temperature fluctuations showing large wave-like motions for Run G7.5wt-02 early in episode (492 sec); vertical axis is height $(0-240 \mathrm{~m})$ and horizontal axis is y-axis $(0-960 \mathrm{~m}$ left to right) and $\mathrm{x}$-axis $(0$ - $960 \mathrm{~m}$ top to bottom), with $\mathrm{y}-\mathrm{z}$ reference plane at $\mathrm{x}=0 \mathrm{~m}$; vertical scale stretched by factor of two for visualization the flow direction is along the $\mathrm{x}$-axis (top to bottom). 


\section{Chapter 7}

\section{Energy Transfer in the SBL}

The transfer of energy between scales is complicated by the presence of wind shear and thermal stratification. Furthermore, there is an exchange between kinetic and potential energy due to effects of buoyancy. In this chapter, the energetics of the evolving SBL are investigated.

\subsection{TKE Transfer}

The reduction of the turbulence in the boundary layer is evident in Figure 6.11, which shows the time history of the vertically-integrated velocity variances by component as the CBL to SBL transition occurs. The preferential reduction of turbulence in vertical velocity component is clearly illustrated as the SBL develops. The variability of turbulence in the SBL is evident, with a period of enhanced turbulence occurring at hour 10.

The transfer of energy between resolved and unresolved (subgrid) scales can be studied with the SGS model used in these simulations. The character of the energy transfer in the CBL and SBL is discussed in Cederwall and Street (1999). There the forward scatter dominated the backscatter terms in the CBL, leading to a relatively large net transfer from resolved to unresolved scales. In contrast, in the SBL, the forward and backscatter terms were more balanced with a small, net transfer to unresolved scales. The vertical profiles of net transfer (dissipation) were similar to those one would obtain using an eddy viscosity approach. Here the analysis is extended to investigate the role of the different stress components in the energy transfer in the SBL, and in particular for the periods before and during the enhanced turbulence event of Run G10.4wt-02T.

The individual component contributions to the energy transfer can be evaluated 
in terms of the dissipation:

$$
\varepsilon_{i, j}^{T K E}=\overline{u_{i} u_{j}} S_{i j}
$$

For the resolved $\leftrightarrow$ unresolved scale transfer of TKE (see Figure 7.1), the 1,3 component is dominant, especially near the ground. The enhanced turbulence leads to a greater forward scatter and a deeper layer of turbulence. Near the ground, the 1,3 component, $\overline{u^{\prime} w^{\prime}}(\partial \bar{u} / \partial z+\partial \bar{w} / \partial x)$, is a primary source for backscatter. This is consistent with analysis of DNS of turbulent channel flow by Härtel and Kleiser (1998), where they found that the correlation of the wall-normal SGS stress with the wall-normal derivative of the resolved streamwise velocity plays a key role in inverse cascade (backscatter) of TKE.

\subsection{Thermal Energy Transfer}

The resolved $\leftrightarrow$ unresolved scale transfer of thermal energy (temperature variance dissipation) poses a challenge for interpretation. Thermal backscatter in atmospheric flows is a relatively new topic.

The individual component contributions to the thermal energy transfer can be evaluated in terms of the dissipation of temperature variance:

$$
\varepsilon_{k}^{\theta}=\overline{u_{k}^{\prime} \theta^{\prime}} \partial \bar{\theta} / \partial x_{k}
$$

As shown in Figure 7.2, the streamwise (1) component, $\left(\overline{u^{\prime} \theta^{\prime}}\right) \partial \theta / \partial x$, is dominant, and becomes very active during the period of enhanced turbulence. Thermal backscatter (negative dissipation of temperature variance) has been reported for the CBL near the ground by Porté-Agel, et al. (1998). They used conditional sampling for analysis of data from an atmospheric field experiment. The thermal backscatter was associated with ejections of warm surface air due to the action of coherent structures in the unstable surface layer. These ejections occurred when there were local decreases in the streamwise velocity. Our finding of the dominance of the streamwise component suggests that coherent structures may be the mechanism for thermal backscatter in the SBL. 
Horizontal (x-y) planes of streamwise velocity and potential temperature are analyzed for coherent structures. A striking example is given in Figure 7.3 for a period just after the enhanced turbulence event. Large-scale structures are evident in both the velocity and temperature fields, which are highly correlated. In regions where the streamwise velocity is decreasing locally, there are cool regions in the temperature. This suggests ejections of cool surface air, analogous to the warm air ejections in the CBL.

To test this suggestion, the two reference cases are explored for instances where cool, slow-moving air is ejected into the flow; i.e. the 'ejection' part of the 'bursting' process. Isosurfaces of $\theta^{\prime}=-0.45 K$ colored by the magnitude of the fluctuating velocity are illustrated in Figure 7.4 for the moderately stable case (Run G7.5wt-02). Blue blobs extending up from the ground are indicators of ejections. Several are seen in the figure. For the more stable case (Run G5wt-02), the blue blobs are also numerous, and are constrained to a more shallow region due to the more shallow SBL depth (see Figure 7.5). For this case, the isosurface value is $\theta^{\prime}=-0.5 K$.

\subsection{Mixing and Stirring Efficiencies}

The transfer of energy between kinetic energy (KE) and potential energy (PE) states is an important part of the energetics of the SBL. Potential energy is increased in the presence of SBL thermal stratification when cool air parcels are moved up into regions of relatively warm air parcels by turbulent motions. The same is true when warm air is moved into cool regions. Following such events, the resulting thermal instability can lead to mixing (diffusion) where the region assumes a new temperature that is a mix of the cool and warm air temperature. This is an irreversible process. Another outcome following such events is that the air parcels return to their previous arrangement (warm above cool), that is, the flow restratifies. This represents a transient, reversible process called stirring (see Tseng, et al., 2001 for a discussion of the distinction between mixing and stirring).

Mixing efficiency is a useful way to quantify the irreversible process of $\mathrm{KE} \leftrightarrow \mathrm{PE}$ exchange in the SBL. It is the ratio of the change of mean potential energy due to a turbulence event to the work done to create the event. The mixing efficiency can be 
defined in terms of the flux Richardson number

$$
R_{f}=\left(g / \theta_{0}\right) \overline{w^{\prime} \theta^{\prime}} /\left(\overline{u^{\prime} w^{\prime}} \partial \bar{u} / \partial z+\overline{v^{\prime} w^{\prime}} \partial \bar{v} / \partial z\right)
$$

The following analogy helps explain the mixing efficiency concept. Suppose that one wanted to mix a stably stratified water tank, and there were a variety of tools to choose from, say a stick, a broom, and a snow shovel. The best tool to use is the one which most efficiently converts the energy imparted by moving the tool in the water to a more well mixed vertical profile (i.e. an increase in potential energy). If most of the energy is dissipated before the mixing can occur (probably the case with a stick), then the mixing efficiency is low. On the other hand, if a fair fraction of the energy is transformed into increased potential energy, then the mixing efficiency is high. The mixing efficiency of a turbulence event is dependent on the fine-scale structure of the turbulence (as expressed above in the flux Richardson number).

Laboratory studies are a good source of data on important mixing processes in stratified flows. For example, a towed grid in a stably stratified tank has an upper limit of $6 \%$ mixing efficiency (Rehmann and Koseff, 2002). In comparison, breaking internal waves have much higher mixing efficiencies (McEwan, 1983; Taylor, 1992), as do shear instabilities (Thorpe, 1981; Koop and Browand, 1979).

Maximum mixing efficiencies for various mechanisms that have been investigated in the laboratory are summarized Table 7.1 adapted from Rehmann (1995, his Table 5.1). The classification of 'Internal' and 'External' follows Turner (1979) who classified external mechanisms as generating turbulence at a boundary (often between fluids of very different densities) and those mechanisms generating turbulence within the interior of a fluid. Turner (1981) speculated that internal mechanisms have selfbalancing mixing processes that regulate the energy supply. These self-regulating processes maintain efficient mixing by keeping the scale of turbulence as large as the stratification will allow. On the other hand, external mechanisms cannot sustain such self-balancing processes and thus optimal turbulence scales are maintained, leading to smaller mixing efficiencies.

Flux Richardson numbers have been evaluated for the two reference runs at hour 8 of the simulations. Values for terms in Equation 7.3 are based on 5-minute averages 
of horizontal averages at each time step. The mixing efficiency for the moderately stable case (Run G7.5wt-02) is generally in the range of $20 \%$ within the SBL, as shown in Figure 7.6. For the strongly stable case (Run G5wt-02), the mixing efficiency is higher (more like $30 \%$ ) within the inversion; there is a maximum very near the ground of $70 \%$, where the surface heat flux has its maximum magnitude (see Figure 7.7). Such a near surface maximum is not seen in the other case. In both cases, the mixing efficiency drops smoothly toward zero with height above the surface-based temperature inversion. The high mixing efficiencies within the surface-based inversion confirm the action of internal mixing mechanisms there, which is expected; the likely mechanism is shear instability. The vorticity has its maximum within the inversion, due to the shear generated by frictional effects of the rough surface; this is discussed further in Section 7.6. The large mixing efficiency that occurs near the ground for the strongly stable case is similar to that observed by Howell and Sun (1999) for nocturnal periods during the 1995 MICROFRONTS experiment in Kansas. Flux Richarson numbers for data at a height of 3 m ranged up to 0.6 (mixing efficiency of $60 \%$ ) for the most stable conditions.

The question arises about the suitability of mixing efficiency to characterize turbulence episodes, such as that described in Section 6.4. To address this, profiles of mixing efficiency (flux Richardson number) for 5-minute periods starting before, during, and after the episode (starting at hour 11, 11.5, and 12, respectively) are shown in Figure 7.8. The mixing efficiency remains relatively unchanged at about $20 \%$ in the lower part of the SBL (within $40 \mathrm{~m}$ of the ground). However, above that, there is a remarkable increase in mixing efficiency during the episode (increasing aloft to nearly $30 \%$ ) compared to that before and after the episode, where the mixing efficiency falls off with height to values close to $5 \%$. The mixing efficiency defined by the flux Richardson number may not be suitable during episodes or in regions of countergradient flux (Tseng, et al., 2001). At best, $R_{f}$ may indicate more correctly the mixing potential rather than the actual mixing.

In contrast to mixing efficiency, the concept of stirring is well suited to transient events. Stirring can be quantified by a stirring length (Tseng, et al., 2001) using the available potential energy (APE). In the study by Tseng, et al. (2001), the APE is obtained as the residual of the total potential energy less the potential energy of 
reference state (the lowest PE state of the flow, as defined by Lorenz, 1955). Since the LES results provide the spatial distribution of temperature, the APE can be obtained directly from

$$
A P E=\frac{g}{\theta_{0} h} \int_{0}^{z=h} \frac{\left\langle\overline{\theta^{\prime 2}}\right\rangle}{\langle\partial \bar{\theta} / \partial z\rangle} d z
$$

where $<>$ represents an average in the horizontal $(\mathrm{x}-\mathrm{y})$ plane. Using the APE and the stratification quantified by the Brunt-Vaisala frequency $N$ averaged over the layer of depth $h$ (see Figure 7.9 for vertical N profile), the stirring length $L_{s}$ is defined by

$$
L_{s}=\sqrt{A P E / N^{2}}
$$

The stirring length is evaluated the episode analyzed in Section 6.4. Statistics of the temperature field for 5-minute periods, from hour 11 to hour 12 of Run G7.5wt-02 which encompasses the episode, are used to calculate stirring lengths. It is useful to assess the stirring length relative to some bounding scale. For these simulations, that bounding scale is the depth $h$ of the SBL. The normalized stirring length is shown in Figure 7.10. The value of 0.05 reached during the episode agrees with typical stirring lengths for a cavity flow when they are normalized by the cavity width (Tseng, et al., 2001). During the episode, the stirring length doubles, reflecting the enhanced stirring due to the increased smaller scale wave-like motions. Those motions die out and the stirring returns to the same level as before the episode.

As discussed earlier, the SBL associated with the episode has a two-part structure, with a dividing height of about $100 \mathrm{~m}$. The stirring lengths are recalculated for the two layers, and shown in Figure 7.11. The onset of the episode is clear in the upper part of the SBL after hour 11.25, reaching a peak value in advance of the time when a smaller peak is reached in the lower SBL. This suggests that the effects of the disturbances are felt first in the upper part of the SBL, and transmitted down into the lower part of the SBL. After the episode, the stirring length in the upper SBL returns to a value smaller than that in the lower SBL.

The mixing efficiency profiles in Figure 7.8 imply that the 'undisturbed' part of the SBL is restricted to a more shallow layer near the ground, i.e. that the dividing height between the disturbed and undisturbed SBL is about $40 \mathrm{~m}$. Using this lower height, the stirring length is recalculated, and plotted in Figure 7.12. Indeed, the 
lower SBL $L_{s}$ is nearly constant during the hour-long period, while the upper SBL $L_{s}$ is much smaller than the near-surface value before and after the episode and much larger during the episode. To provide some comparison with the behavior of the mixing efficiency, values of $R_{f}$ for each 5-minute period were averaged vertically for the same two layers. The time history of these layer-averaged $R_{f}$ values are shown in Figure 7.13, and are consistent with the profiles in Figure 7.8. As expected, the average mixing efficiency near the ground is quite constant at about $20 \%$. The average mixing efficiency above $40 \mathrm{~m}$ is near $5 \%$ before and after the episode, and approaches a maximum of $30 \%$ during the episode. Compared to the stirring length, the relative variation before, during, and after the episode is greater for the mixing efficiency.

The stirring length applied to these results provides a more robust measure of the effects of the turbulent motion in moving patches of air to regions of different temperature (i.e. stirring). The result is an occasional transient period of countergradient flux and thermal instability. In contrast, the mixing efficiency (defined by $R_{f}$ ) mimics the potential for mixing (much like the stirring length) associated with transient events, but does not capture the mixing associated with diffusion.

\subsection{Waves versus Turbulence in the SBL}

The episode analyzed in Section 6.4 has wave-like undulations in the upper part of the SBL that contribute to the increased velocity variance, turbulent stress, and heat flux. An important question is whether the enhanced 'turbulence' is really turbulence alone, or more correctly enhanced 'variability' caused by a combination of turbulence and gravity waves.

A proven technique for studying flows having both turbulence and waves is the spectral analysis of the vertical motion and temperature. In particular, the crossspectral phase angle gives the phase difference between the times series of vertical motion and temperature. Rees, et al. (2001) have used this approach in the study of waves in the SBL over an Antarctic ice shelf. Holt, et al. (1992) and Briggs, et al. (1998) used this approach in the analysis of wave motions in their DNS results. Using the co-spectrum $(\mathrm{Co})$ and quadrature spectrum $(Q u)$, the phase angle $(\phi)$ can 
be expressed as (Lumley and Panofsky, 1964)

$$
\tan (\phi)=\frac{Q u_{w \theta}}{C o_{w \theta}}
$$

If the phase angle $\phi=0, w$ and $\theta$ are in phase and the turbulent fluxes are downgradient. If $\phi= \pm \pi$, the fluxes are countergradient. If $\phi= \pm \pi / 2$, internal wave motions are present.

Phase angles have been calculated for 8 different heights during the hour-long period encompassing the turbulent episode described in Section 6.4. A simulated tower was placed in the domain at the midpoint in the $\mathrm{x}$ and $\mathrm{y}$ direction. Sampling every time step was conducted at heights of 15, 45, 75, 105, 135, 165, 195, and $225 \mathrm{~m}$ for 10 minutes around the episode peak. The 10-minute time series of $w$ and $\theta$ were divided into four 2.5-minute segments which were analyzed spectrally to obtain the co-spectrum and quadrature spectrum. The vertical regimes of the episodic SBL are highlighted by the phase angle analysis. In the layer near the surface (45 m sampling height) where the effect of the episode is very weak as reflected in fairly steady mixing efficiencies and stirring lengths, down-gradient turbulent flux is dominant at nearly all frequencies, except the very lowest ones (see Figure 7.14). Further up into the SBL near the dividing height (105 m sampling height) used to distinguish the lower and upper SBL in Section 6.4, the phase angles in Figure 7.15 show some countergradient flux and wave activity at the lowest frequencies. At higher frequencies, there is increasing scatter of $\phi$ with frequency between $\phi=0$ and $\phi= \pm \pi / 2$; delineation of waves and turbulence is not very clear. In the upper part of the SBL (135 m sampling height), countergradient flux is dominant in the middle frequencies. There is scatter at the lowest and highest frequencies (see Figure 7.16), with a weak clustering of phase angles near $\phi=-\pi / 2$ near $5 \mathrm{~Hz}$. Although the character of the phase angle distribution varies in a systematic way from the lowest part of the SBL (with downgradient flux) to the upper part (with countergradient flux), there are no patterns that point out strong regions of waves.

There is one last analysis that may shed light on the possible behavior of waves in the episodic period. The Scorer parameter has been used to delineate waves into external and internal waves (Nappo and Chimonas, 1992). This parameter is obtained 
as part of the solution for linear waves in a continuously stratified Boussinesq flow, which satisfies the Taylor-Goldstein equation. The Scorer parameter $\left(l_{s}\right)$ is defined by

$$
l_{s}^{2}=\frac{N^{2}}{u^{2}}-\frac{\left(\partial^{2} u / \partial z^{2}\right)}{u}
$$

For a given wavenumber $k$, gravity waves are external if $l_{s}^{2}<k^{2}$, and internal if $l_{s}^{2}>k^{2}$. Partitioning waves into these two types is important in understanding the energetics of the SBL since external waves cannot propagate momentum vertically, while internal waves can. Rees and Rottman (1994) used the Scorer parameter in their analysis of waves over the Antarctic Ice Shelf to identify layers where vertically propagating waves are trapped. In the polar SBL, they found that both the stratification and velocity curvature terms are important in Equation 7.7.

The Scorer parameter is shown for the SBL for 10-minute period during the episode in Figure 7.17. The profile of the Scorer parameter during the episode is well organized, eventually becoming negative above the SBL top. Small scale variability in the profile comes mainly from the vertical second derivative of the velocity, which represents the curvature of the velocity profile. What are the wavenumbers for wavelengths of interest during the episode? The average number of streamwise waves in the episode in the upper part of the SBL is about 6 , which implies an average $\lambda=160 \mathrm{~m}$. The value of $k^{2}$ for $\lambda=160 \mathrm{~m}$ is 0.0015 , which is just beyond the maximum $l_{s}^{2}$ value for the plot in Figure 7.17. This implies that the wave-like undulations during the episode are external waves. There are two vertical lines in Figure 7.17 indicating the wavenumbers associated with wavelengths of $600 \mathrm{~m}$ and $1150 \mathrm{~m}$. The values are chosen as the minimum wavelengths for waves to be of the internal type and thus to be capable of propagating momentum vertically throughout the lower SBL (heights below $100 \mathrm{~m}$ ) and upper SBL, respectively. Note that waves with wavelengths greater than $235 \mathrm{~m}$ would be considered internal waves in the layer near the ground where the mixing and stirring are steady during the event, as discussed in Section 7.3. 


\subsection{Coherent Structures in the SBL}

Coherent structures in turbulent flow have been studied experimentally, as summarized in Section 2.2. Often the statistical description of turbulence will mask the coherent motions that reside in the flow. These coherent motions are important in the production and dissipation of the turbulence. The challenge is to identify the coherent structures.

There are several techniques that have been used over the years to identify coherent structures and vortex cores. Two promising approaches have been advanced by Chacin and Cantwell (1997) and by Jeong et al. (1995). In the first approach, eigenvalues and invariants of the velocity tensor are used to characterize the flow field and tag vortex cores. The second approach uses the second largest eigenvalue $\left(\lambda_{2}\right)$ of the tensor $S_{i k} S_{k j}+\Omega_{i k} \Omega_{k j}$. Vortices are identified by isosurfaces of negative values of $\lambda_{2}$.

The $\lambda_{2}$ method is applied to velocity fields for Run G7.5wt-02. As seen in Figure 7.18, there are numerous short vortex cores visualized, especially near the ground (colored by blue). These near-surface structures are indicative of coherent motions which could be associated with the 'bursting' phenomena. The orientation of the vortex cores is not uniformly aligned with the mean flow, which is from bottom to top in the figure. For the more stably stratified case, Run G5wt-02, the vortex cores identified by the $\lambda_{2}$ method are more uniformly aligned, as seen in Figure 7.19. The alignment is normal to the geostrophic forcing. Note that the flow in the vicinity of the vortex cores is from the bottom to top in the figure. The geostrophic forcing is rotated about 30 degress clockwise from the lower level winds. In the presence of strong stability and light winds, the relative importance of the geostrophic forcing is greater. For the less stable case in Figure 7.18, the increased wind speed and deeper SBL (with weaker surface-based inversion) lead to less organized arrangement of vortex cores. 


\subsection{Structural Regimes of the SBL}

The SBL has distinctly different regimes within and above the surface-based temperature inversion. The lower regime has more chaotic, small scale structure in the velocity field, while the upper regime has larger scale structure that are more wave-like. This is illustrated for the strongly stable case at fine scale (Run G5wt-02F).

A x-z plane of fluctuating velocity vectors is shown in Figure 7.20. In the front-on view (upper right), the u' and w' components are evident, but the v' components are in and out of the plane. To facilitate seeing the 3-dimensional nature of the velocity field, the $\mathrm{x}-\mathrm{z}$ plane is rotated in the figure such that the left edge passes in front of the viewer to provide 3 more perspectives. There are clearly two different velocity regimes, with the dividing line about the 6th level up from the bottom. To reinforce this, the $\mathrm{x}-\mathrm{z}$ plane is plotted again with fluctuating velocity vectors, but this time with the plane colored by the v' value. The large-scale structures in the v' field that could be seen in Figure 7.20 are now quite evident in Figure 7.21.

Visually, the lower regime is characterized by small-scale motions while the upper domain is characterized by larger-scale motions. Spectral analyses were performed for Run G5wt-02F, and are shown in Figure 7.22. Spectra are shown for three levels that sample the lower regime (level 4), just above the dividing level (level 8), and well into the upper regime (level 15). What is immediately obvious is that energy at the small scales decreases markedly as one goes from the lower regime to the upper regime. What is a little less clear is that the energy in the upper regime is larger in the larger scales (lower wavenumber). This is most true for the energy in the v-component of velocity.

The two regimes are tied to the thermal structure. To illustrate this, The fluctuating velocity field is plotted on a vertical cross section of potential temperature in the $\mathrm{x}-\mathrm{z}$ plane, shown in Figure 7.23. The transition between regimes occurs in the upper portion of the surface-based inversion. It is the combination of surface-generated turbulence and buoyancy effects of the thermal stratification that modulate this structure. The lower regime is characterized by the presence of vorticity, while the upper regime is distinctly lacking in vorticity (see Figure 7.24). What vorticity there is in the upper regime is primarily in the y-component, as seen in Figure 7.25. This 
is related to the wave-like motions that dominate the upper regime and are aligned with the spanwise direction. Andren (1995) found a similar two-part structure in his results of LES of the SBL. 
Table 7.1: Summary of Mixing Efficiencies for Various Mixing Mechanisms (after Rehmann, 1995).

\begin{tabular}{|l|l|r|}
\hline Mechanism & Classification & Maximum ME \% \\
\hline \hline Breaking internal waves & Internal & 35 \\
\hline Shear instability & Internal & $20-35$ \\
\hline Vortex rings & External & 21 \\
\hline Wake & External & 16 \\
\hline Turbulent "blobs" & External & 16 \\
\hline Dropped grid & External & 12 \\
\hline Towed grid & External & 5 \\
\hline
\end{tabular}
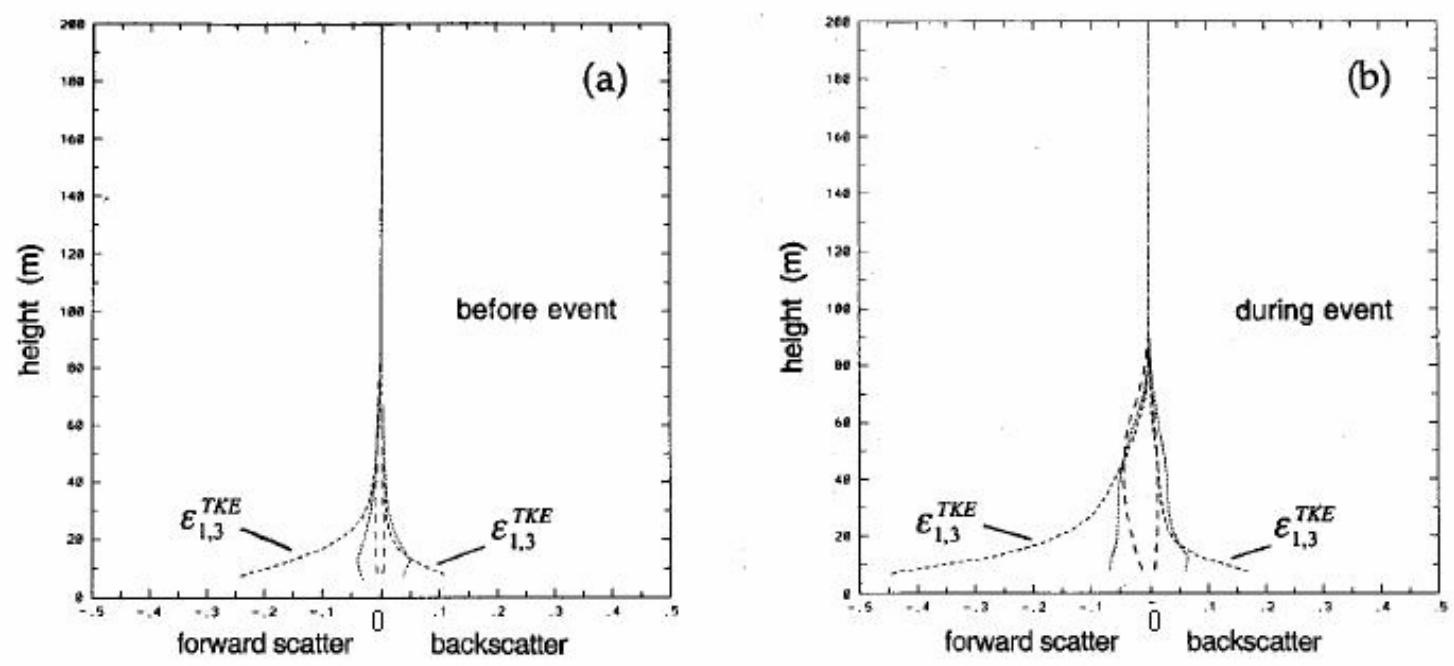

Figure 7.1: Vertical profiles of SGS TKE transfer by component (1,2: dotted line; 1,3: short dashed line; 2,3: long-dashed line) for periods (a) before and (b) during enhanced turbulence; vertical axis is 0 to $200 \mathrm{~m}$, and horizontal axis is -0.5 to $0.5 \times 10^{-2} \mathrm{~m}^{2} / \mathrm{s}^{3}$. 

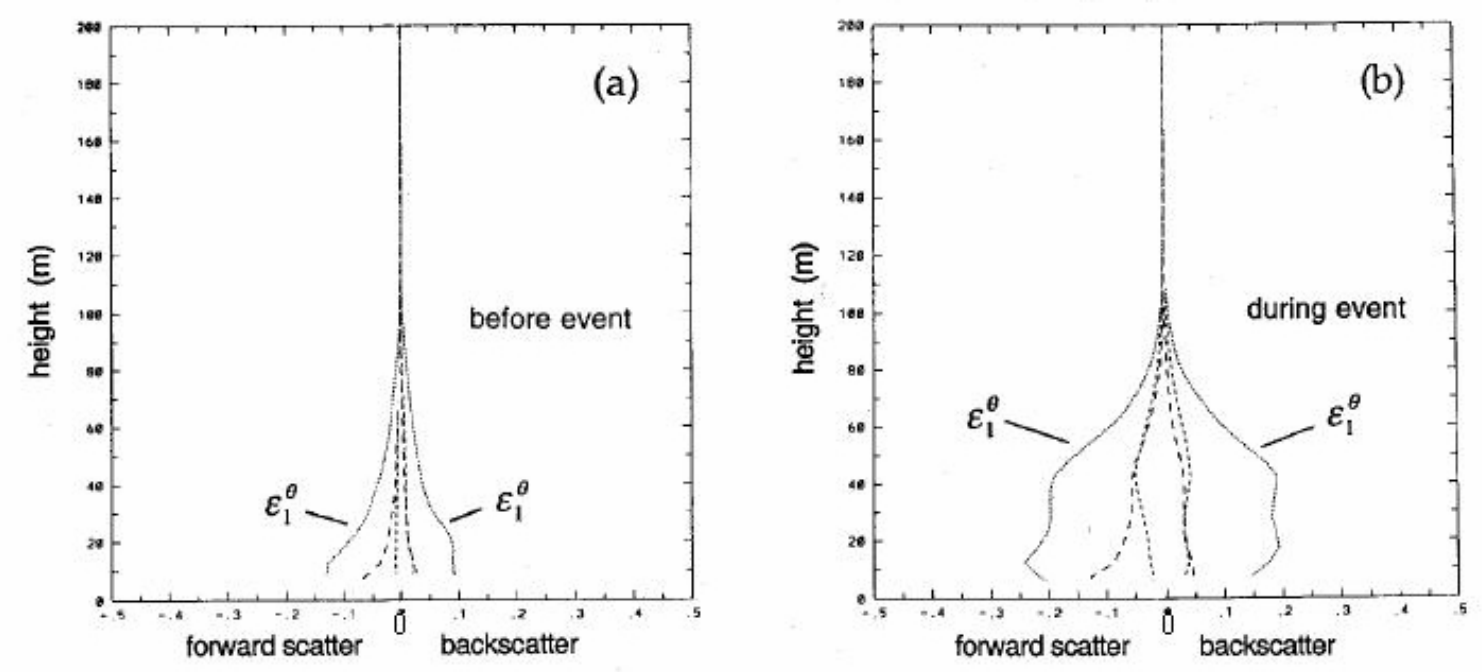

Figure 7.2: Vertical profiles of thermal energy transfer by component (1: dotted line; 2: short dashed line; 3: long-dashed line) for periods (a) before and (b) during enhanced turbulence; vertical axis is 0 to $200 \mathrm{~m}$, and horizontal axis is -0.5 to $0.5 \times 10^{-2} \mathrm{~K}^{2} / \mathrm{s}$.
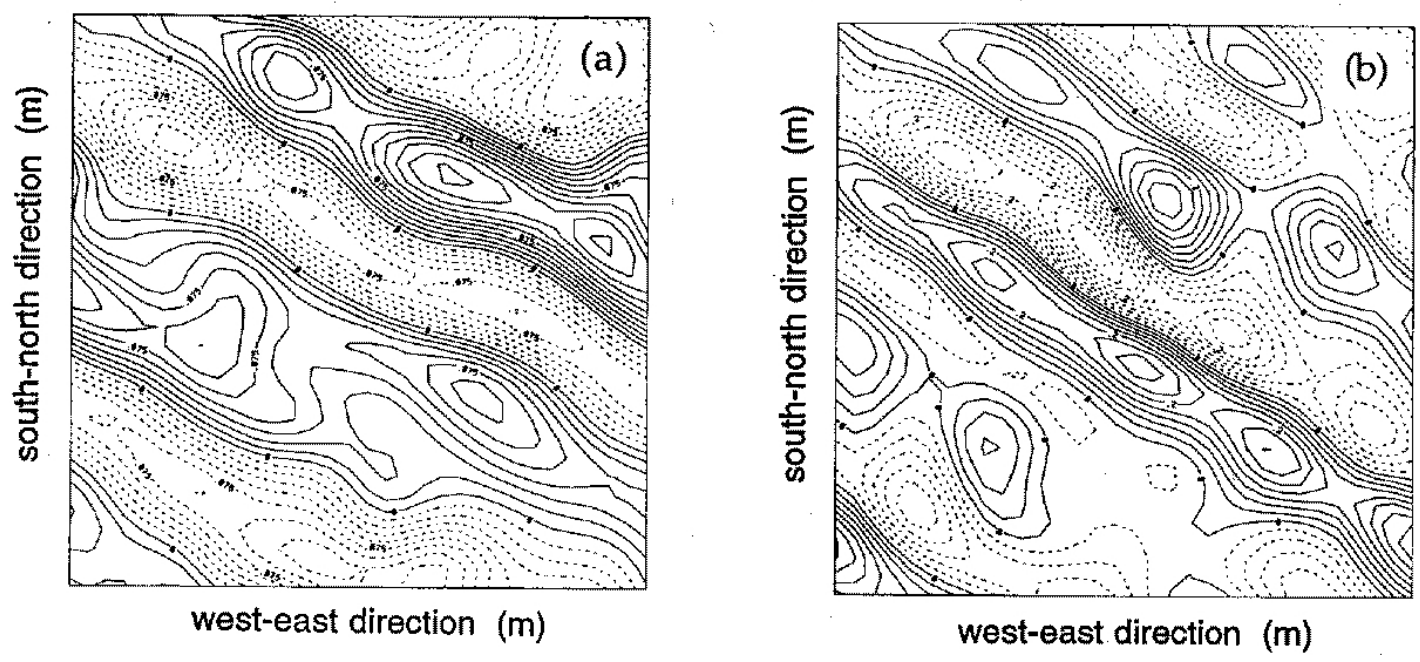

Figure 7.3: Patterns of (a) fluctuating streamwise velocity and (b) fluctuating potential temperature, with mean values of $1.9 \mathrm{~m} / \mathrm{s}$ and $280.8 \mathrm{~K}$, respectively; contour values: 0.015 $\mathrm{m} / \mathrm{s}$ and $0.04 \mathrm{~K}$; mean flow is left to right. 


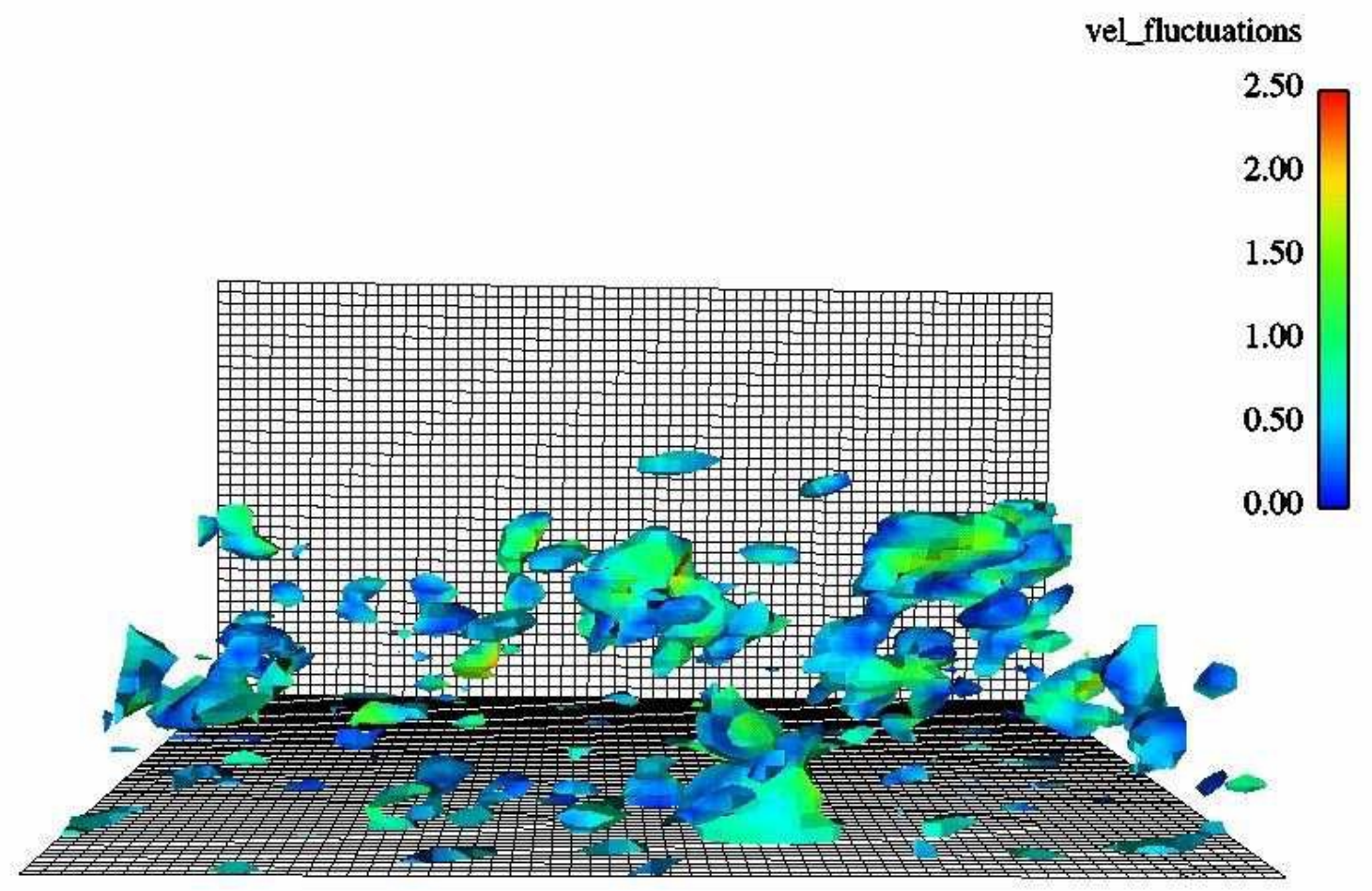

Figure 7.4: Isosurfaces of $\theta^{\prime}=-0.45 K$ colored by magnitude of fluctuating velocity for Run G7.5wt-02 at hour 8; vertical axis is height $(0-240 \mathrm{~m})$, and horizontal axis (left right) is west - east direction $(0-960 \mathrm{~m})$. 


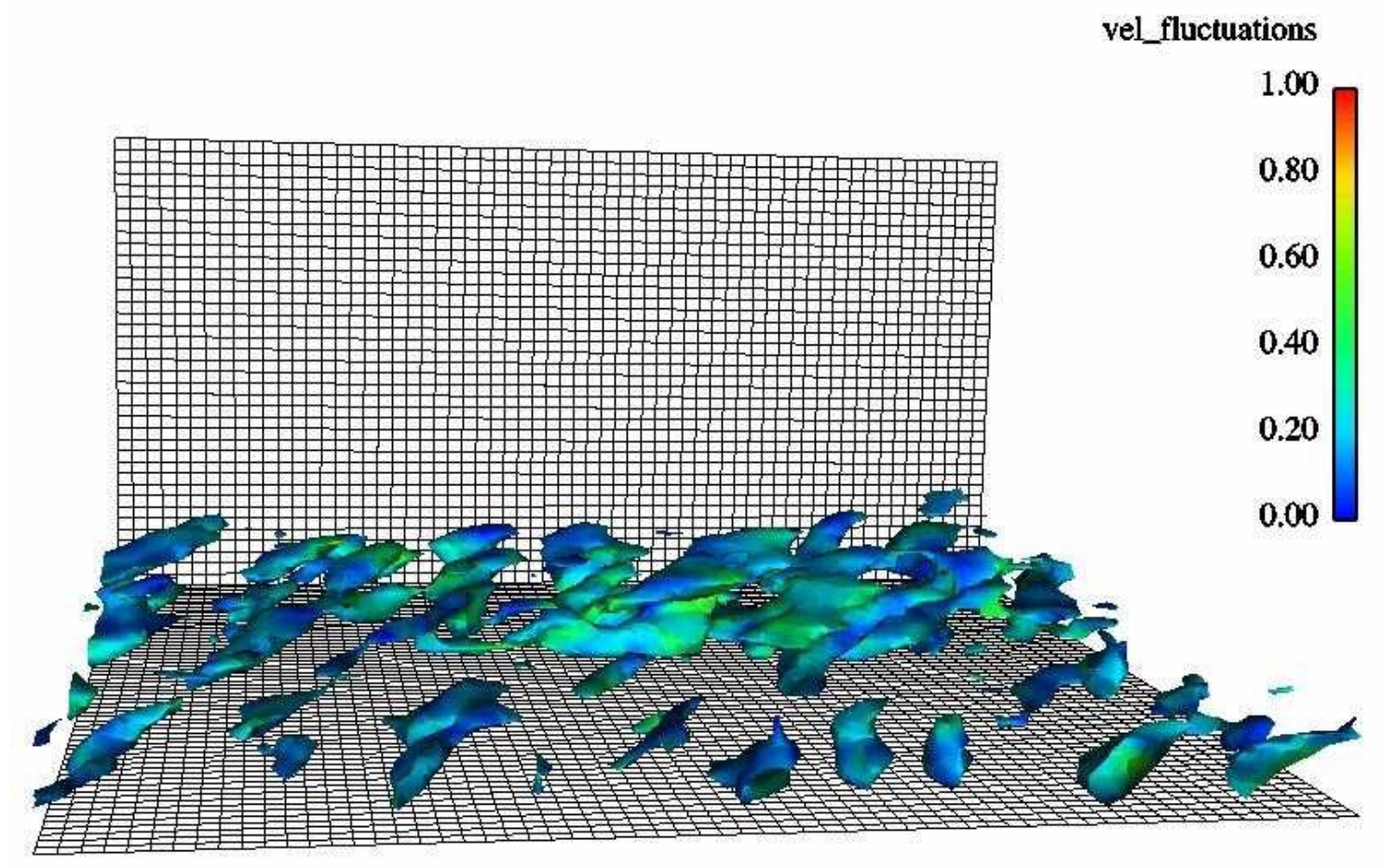

Figure 7.5: Isosurfaces of $\theta^{\prime}=-0.5 K$ colored by magnitude of fluctuating velocity for Run G5wt-02 at hour 8; vertical axis is height $(0-240 \mathrm{~m})$, and horizontal axis (left - right) is west - east direction $(0-960 \mathrm{~m})$. 


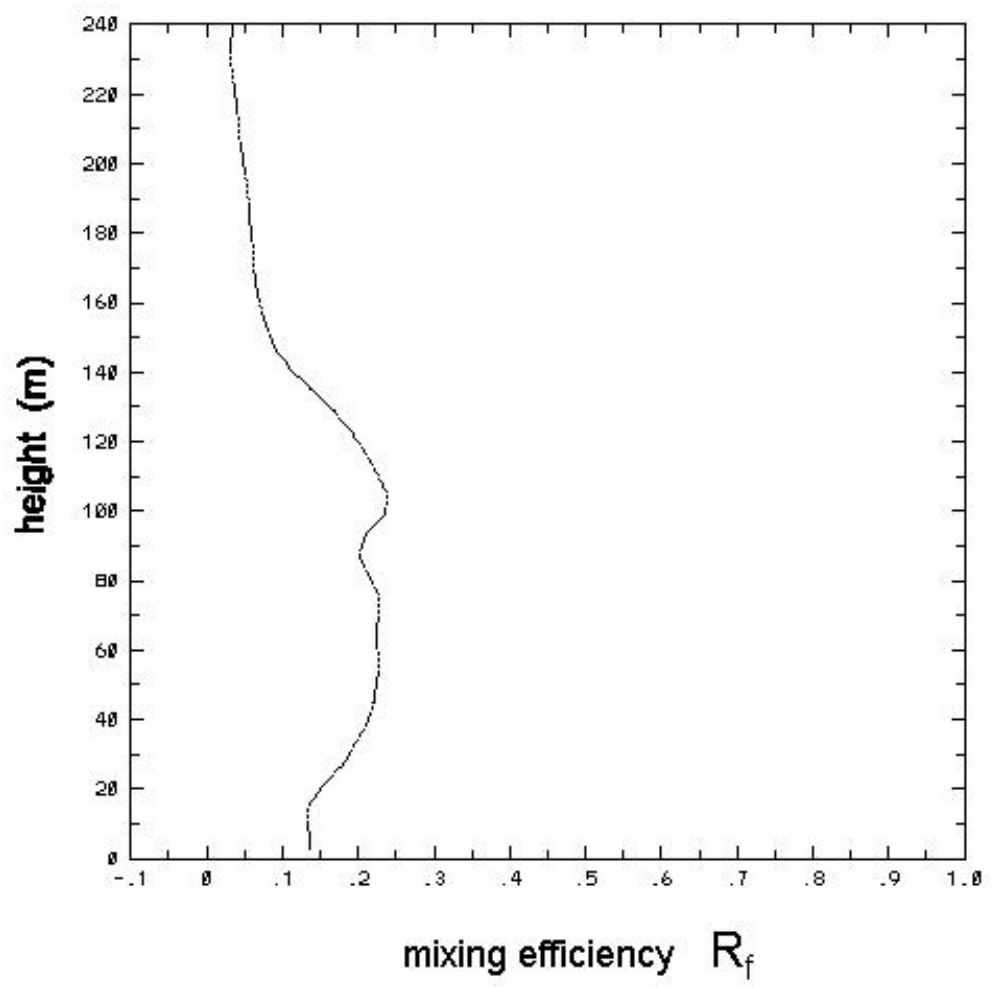

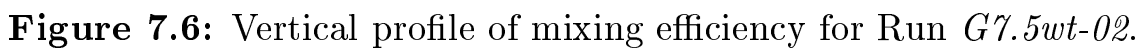




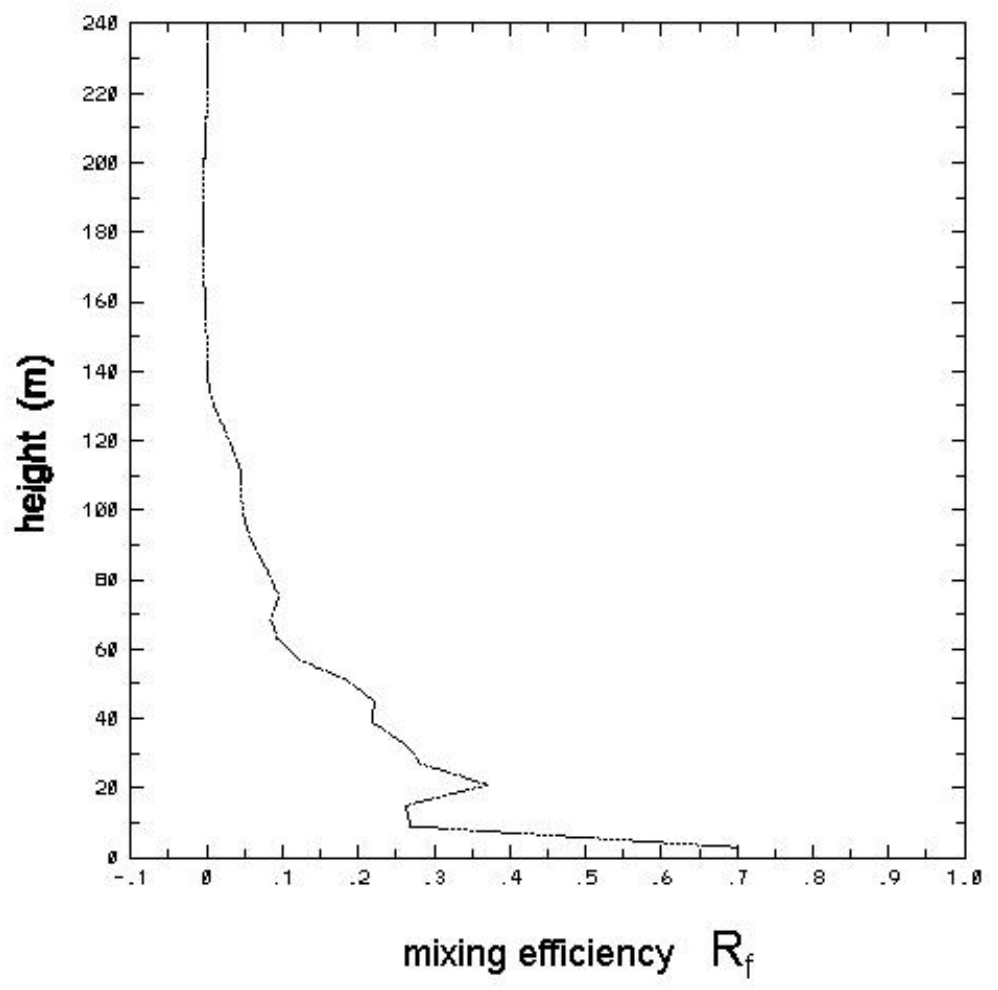

Figure 7.7: Vertical profile of mixing efficiency for Run G5wt-02. 


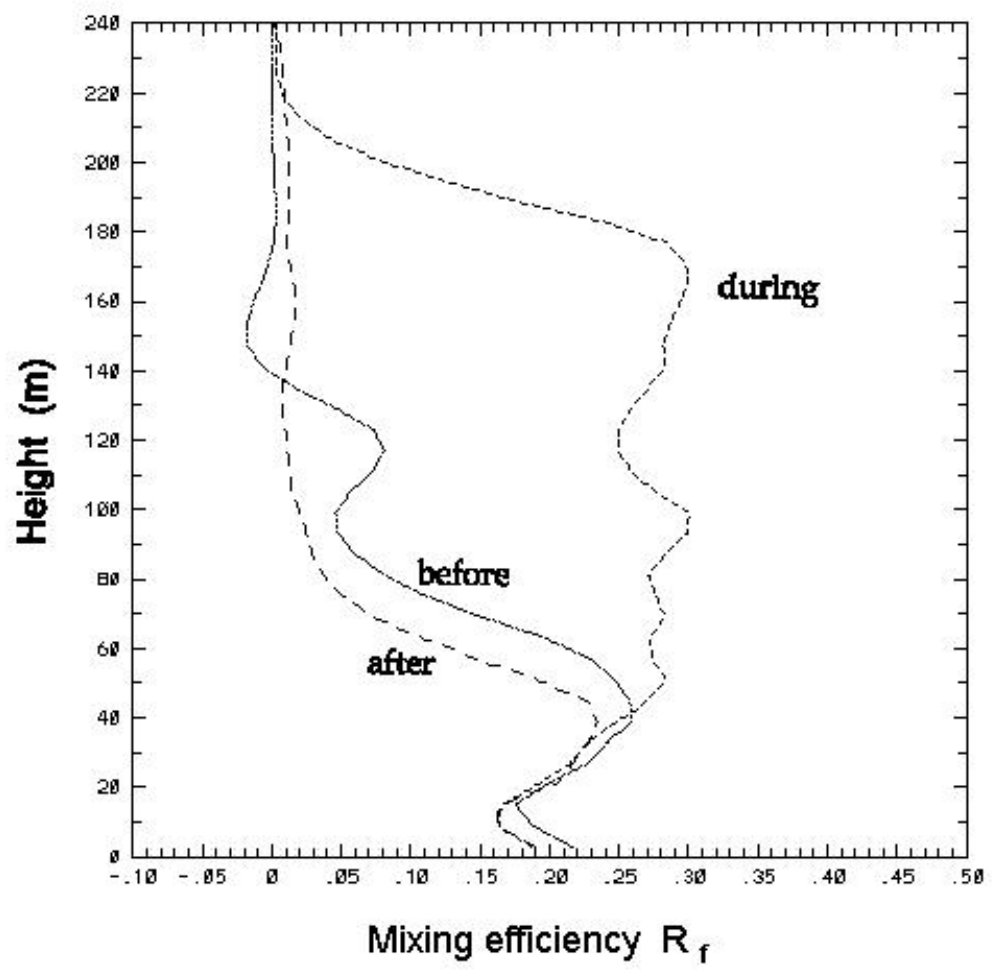

Figure 7.8: Vertical profiles of mixing efficiency before, during, and after episode described in Section 6.4. 


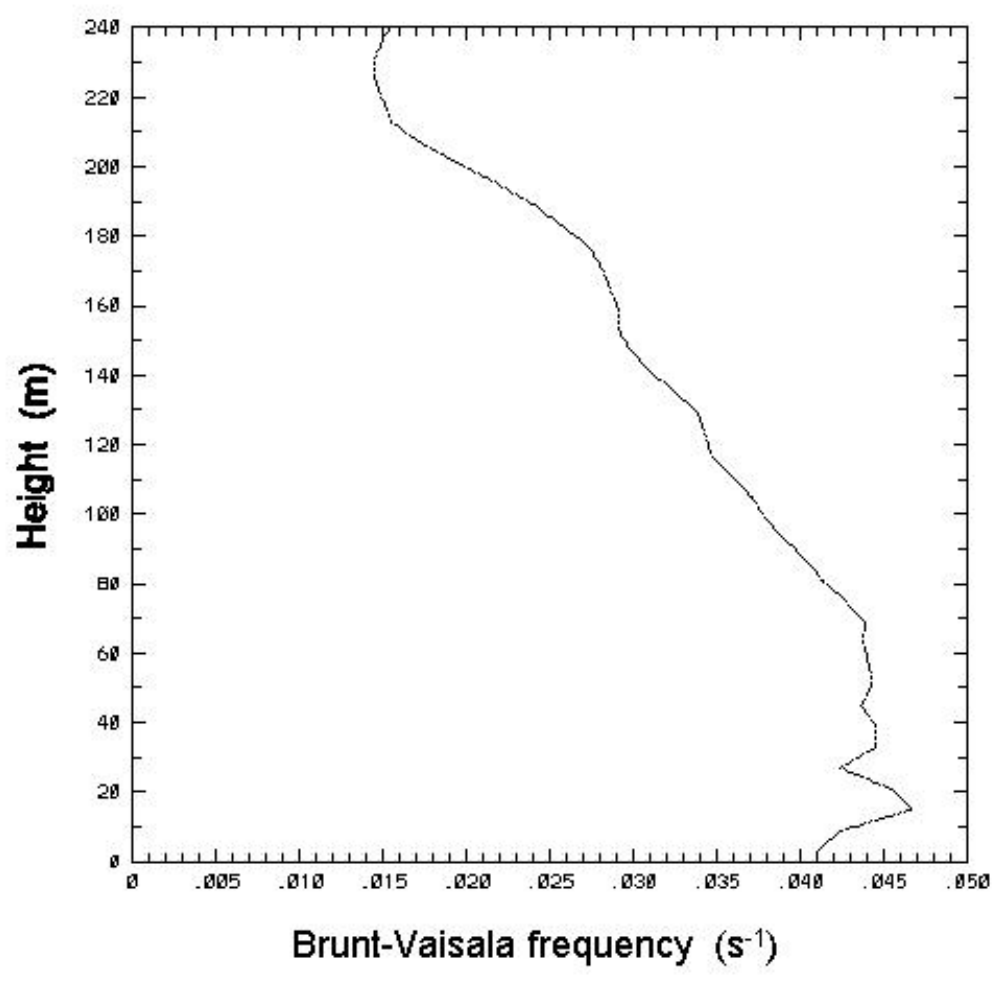

Figure 7.9: Brunt-Vaisala frequency during episode in Run G7.5wt-02. 


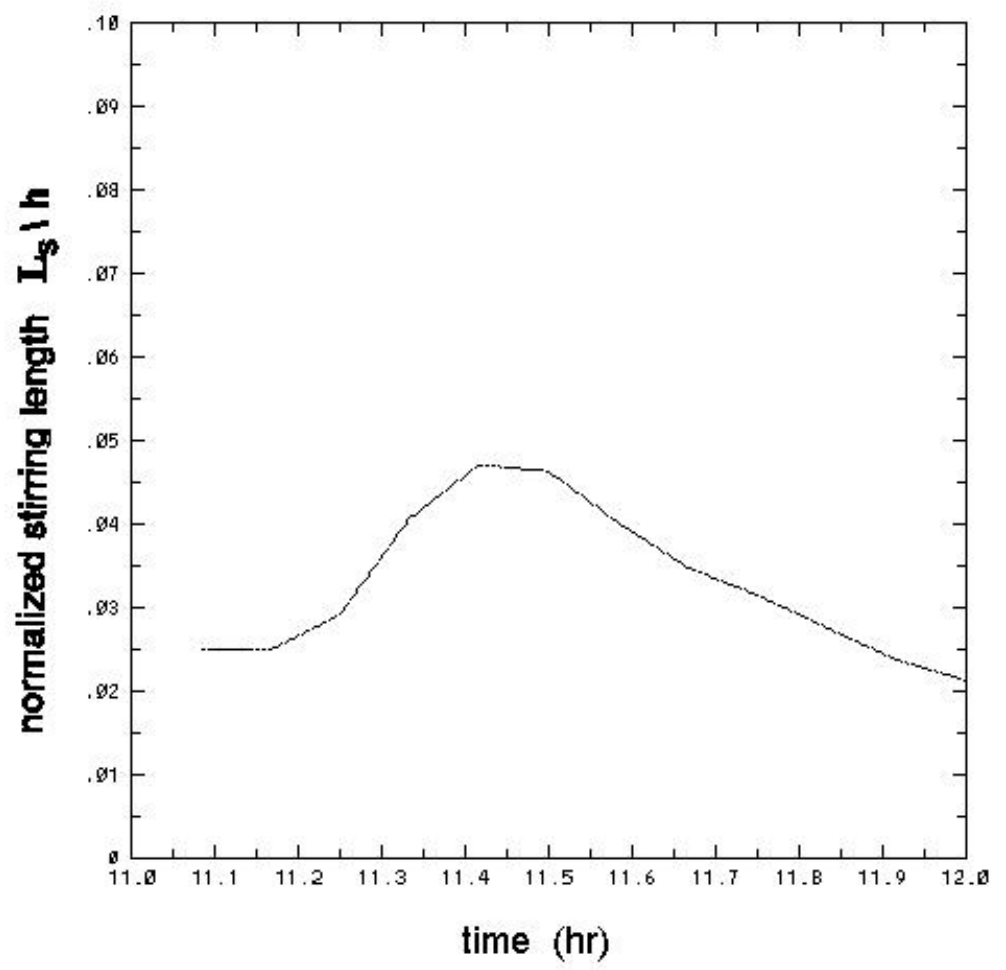

Figure 7.10: Stirring length normalized by SBL depth $(h)$ for period in Run G7.5wt-02 encompassing episode described in Section 6.4. 


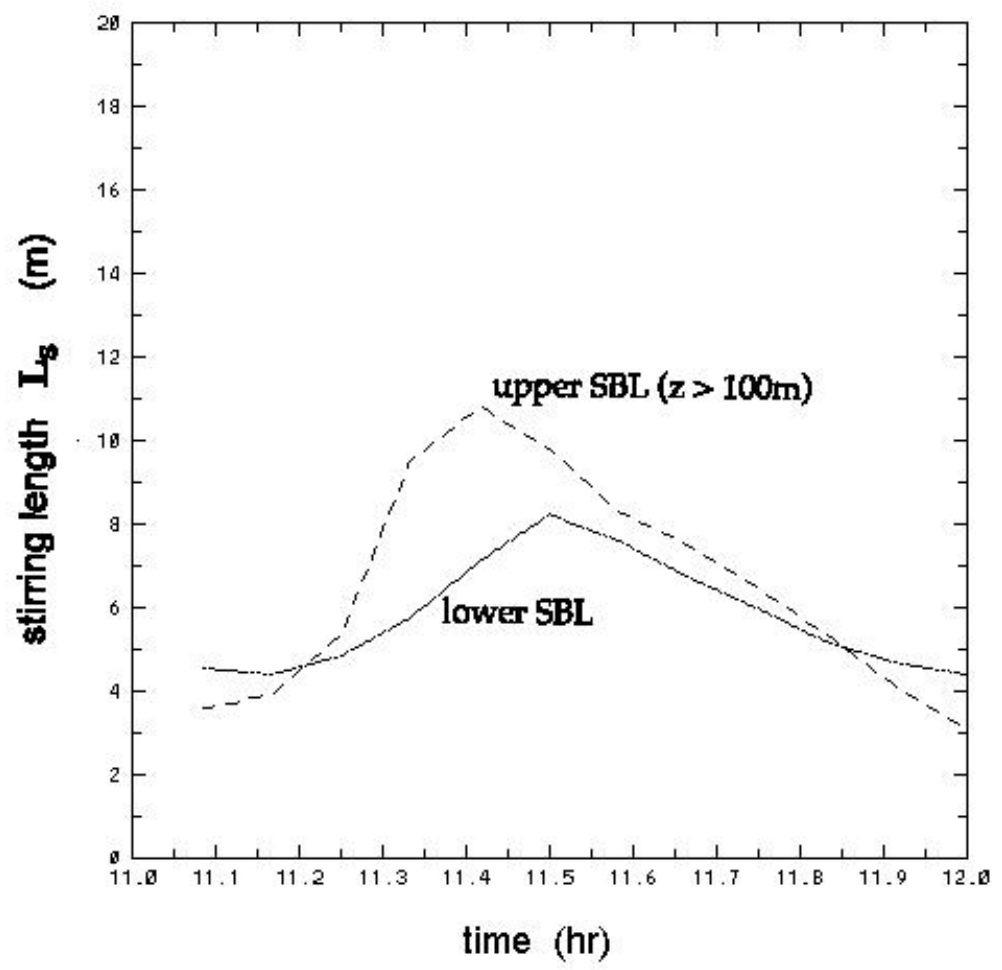

Figure 7.11: Stirring lengths in lower and upper (>100m) SBL for period in Run G7.5wt02 encompassing episode described in Section 6.4. 


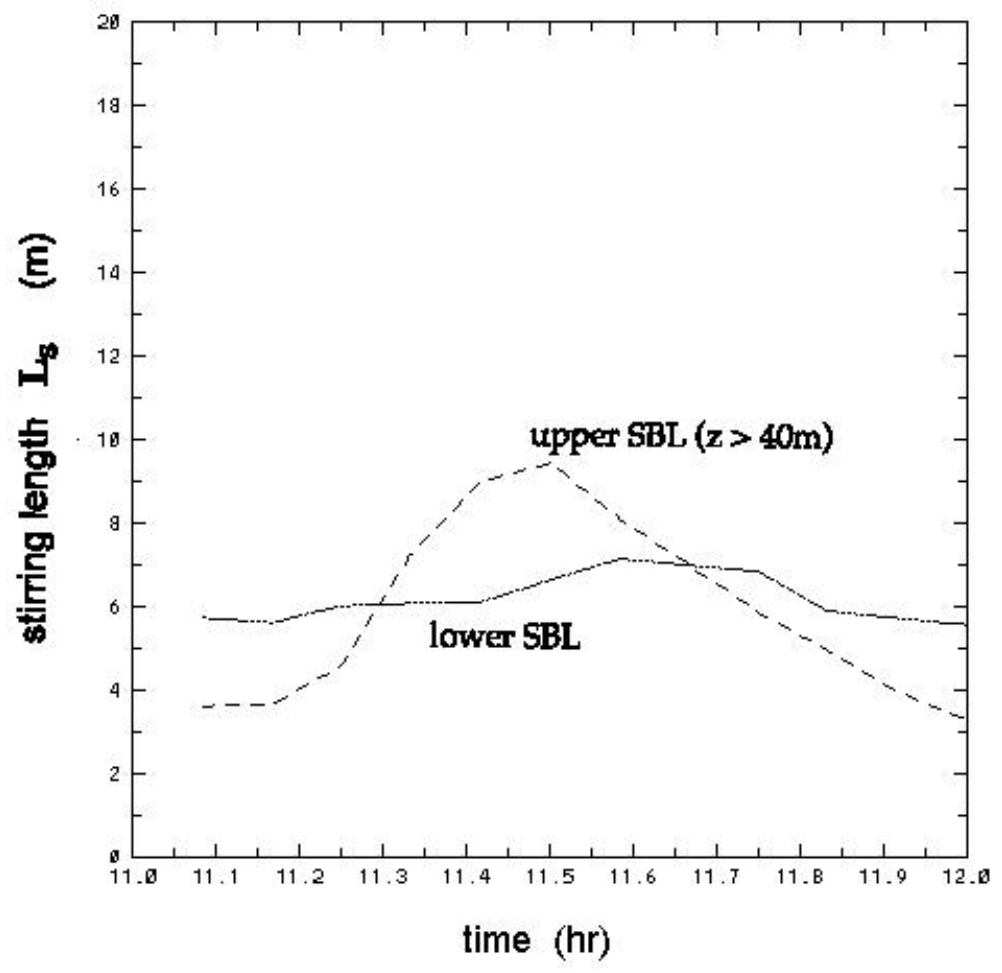

Figure 7.12: Stirring lengths in lower and upper (>40m) SBL for period in Run G7.5wt-02 encompassing episode described in Section 6.4. 


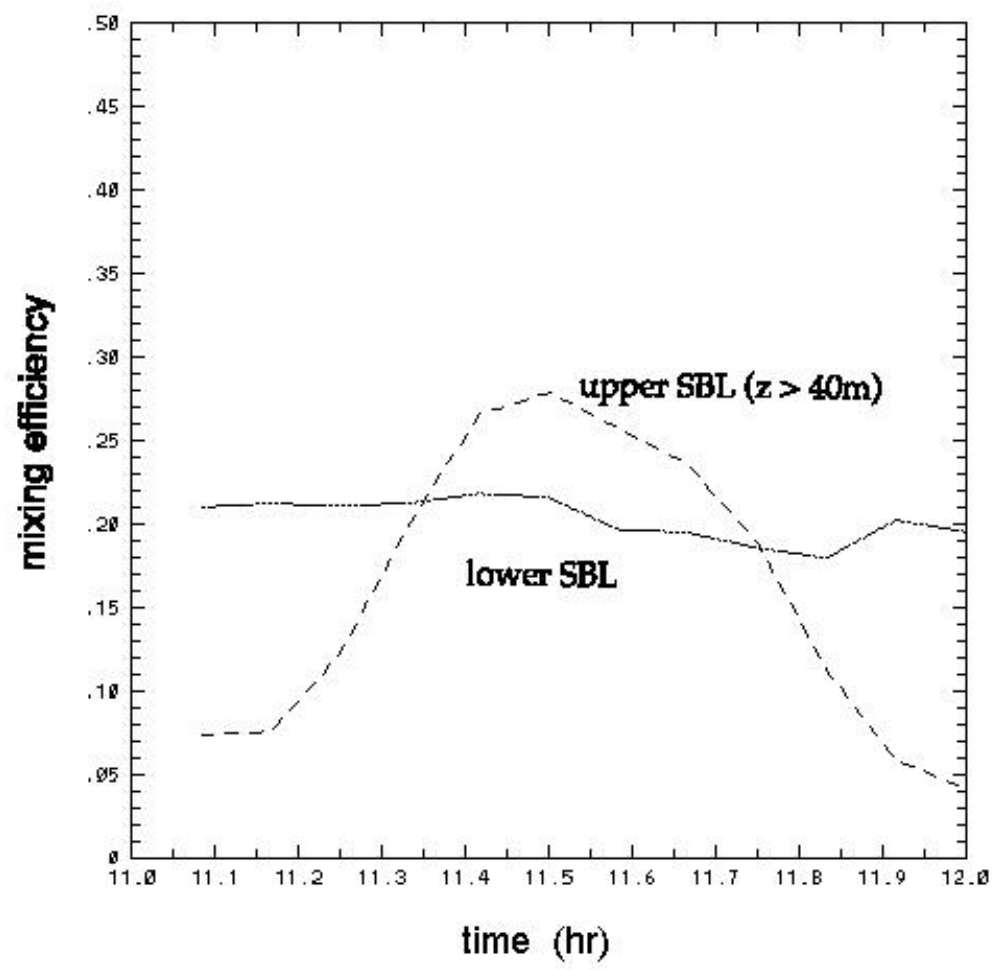

Figure 7.13: Mixing efficiency averaged over two layers in the lower and upper $(>40 \mathrm{~m})$ SBL for period in Run G7.5wt-02 encompassing episode described in Section 6.4. 


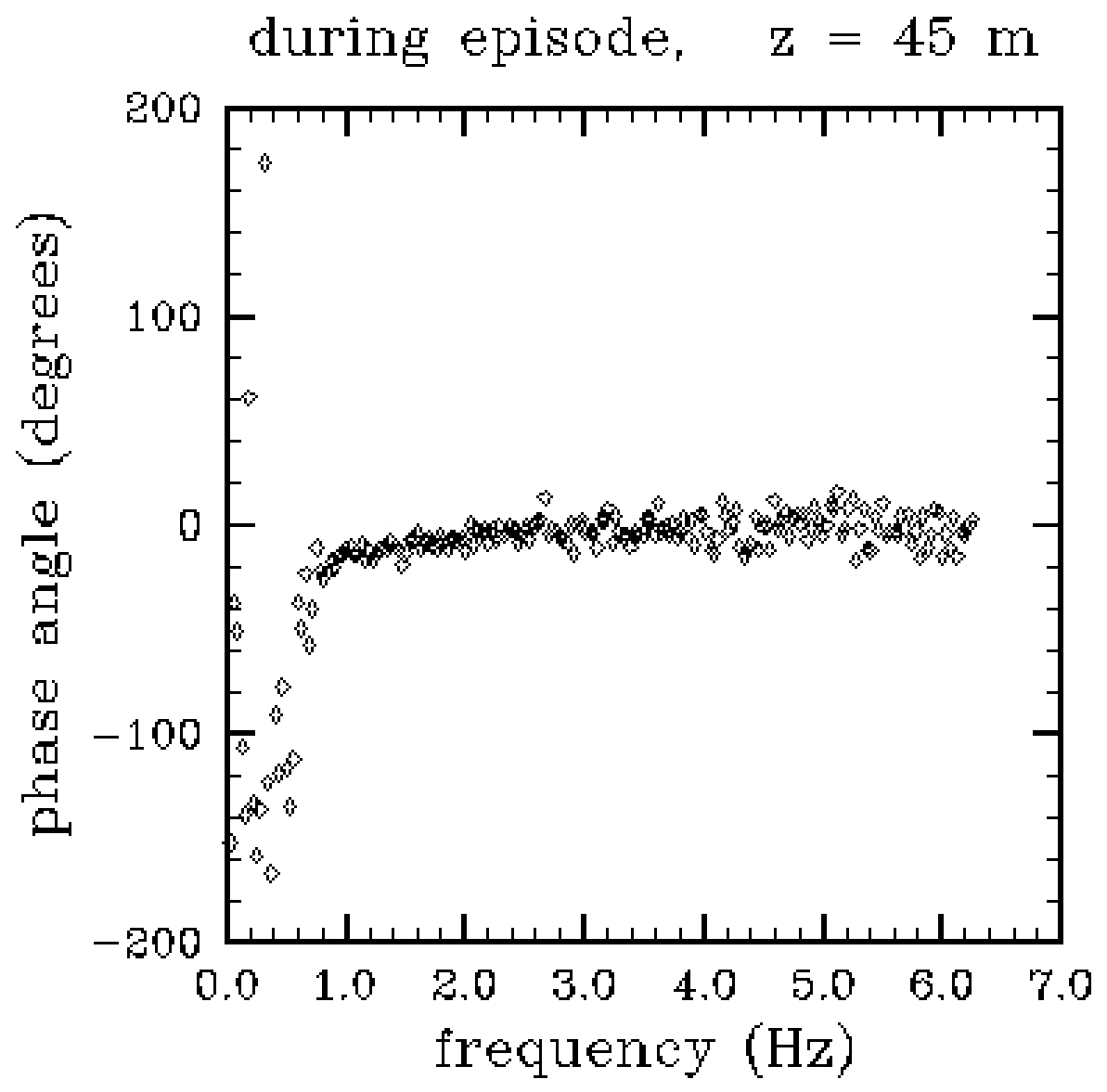

Figure 7.14: Phase angles obtained from $C o_{w \theta}$ and $Q u_{w \theta}$ at 45-m height for 10-minute period during episode described in Section 6.4. 


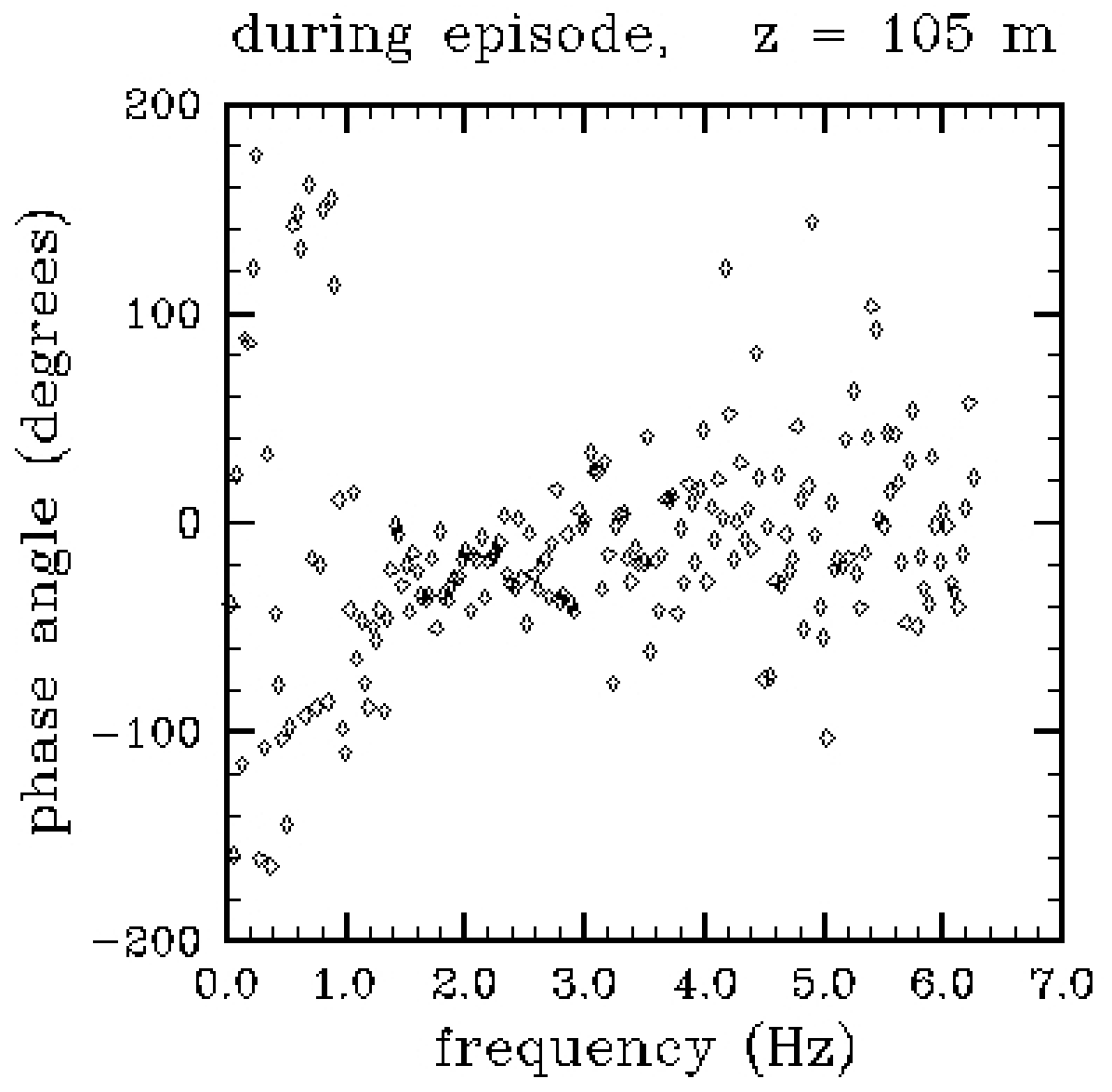

Figure 7.15: Phase angles obtained from $C o_{w \theta}$ and $Q u_{w \theta}$ at 105-m height for 10-minute period during episode described in Section 6.4. 


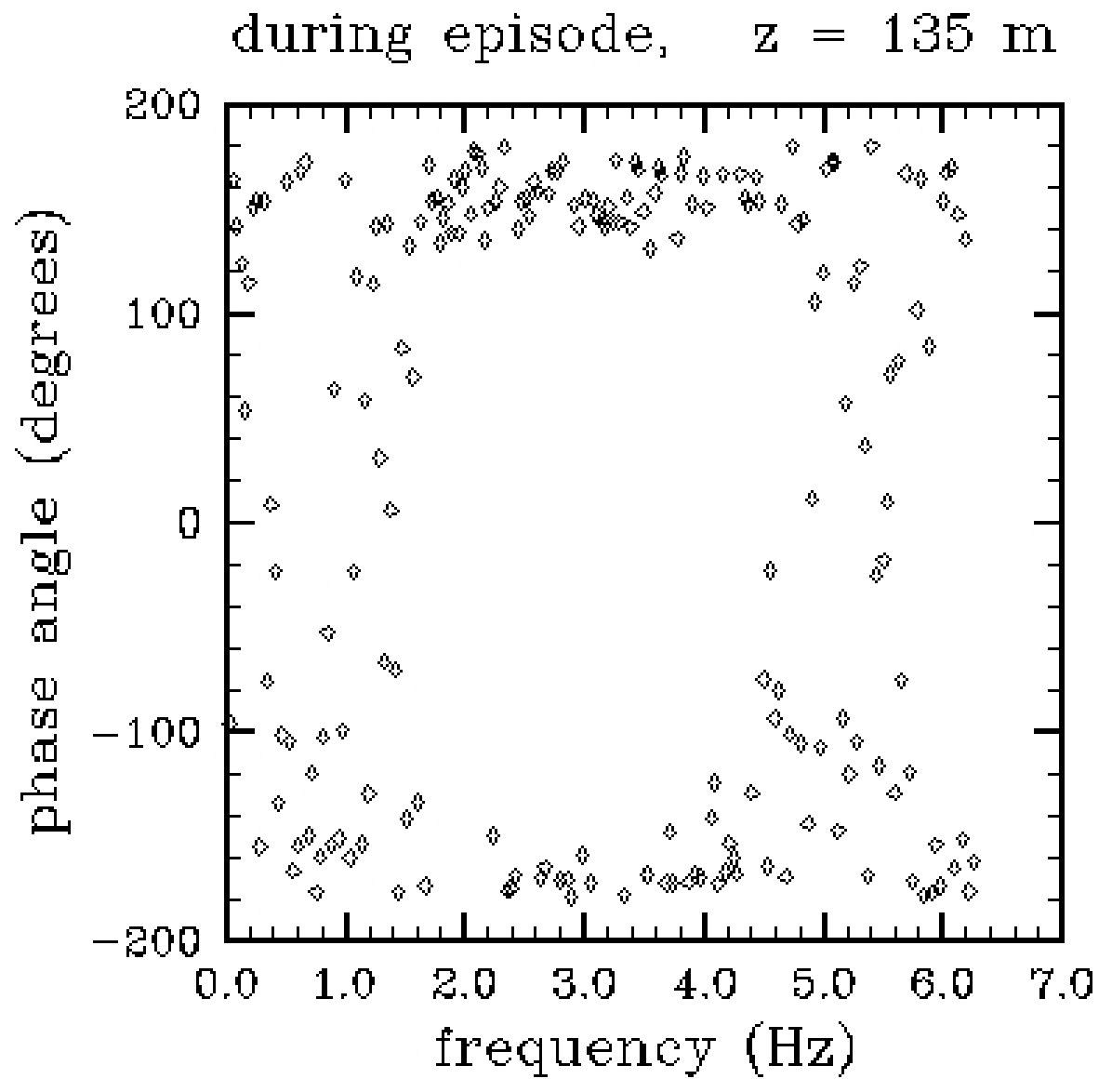

Figure 7.16: Phase angles obtained from $C o_{w \theta}$ and $Q u_{w \theta}$ at $135-\mathrm{m}$ height for 10-minute period during episode described in Section 6.4. 


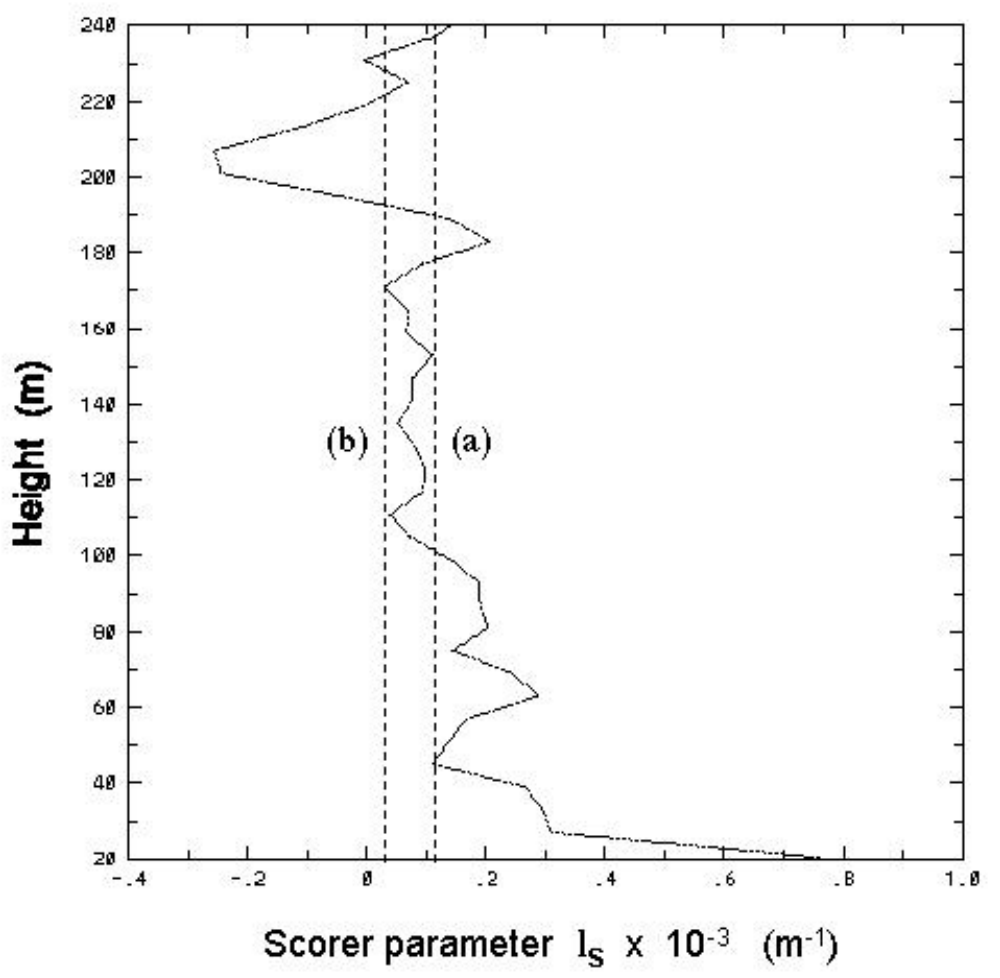

Figure 7.17: Scorer parameter for 10-minute period during episode described in Section 6.4; vertical dashed lines are for wavenumbers associated with (a) $\lambda=600 \mathrm{~m}$, and (b) $\lambda=1150 \mathrm{~m}$. 


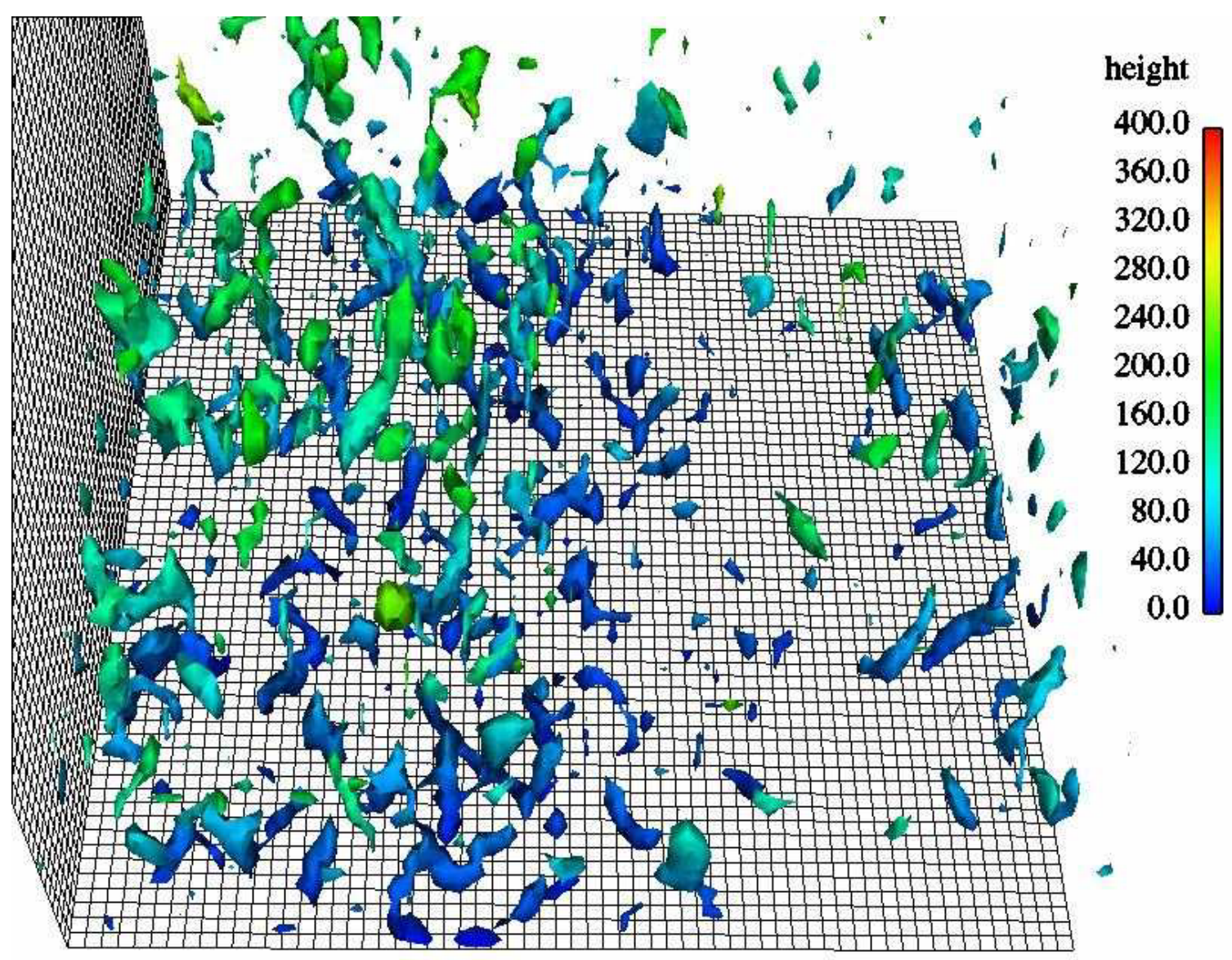

Figure 7.18: Isosurfaces of $\lambda_{2}=-0.001$ colored by height for Run $67.5 w t-02$ at hour 8; flow is from bottom to top; horizontal axes are south-north (right-left: $0-960 \mathrm{~m}$ ) and west-east (bottom-top: $0-960 \mathrm{~m}$ ). 

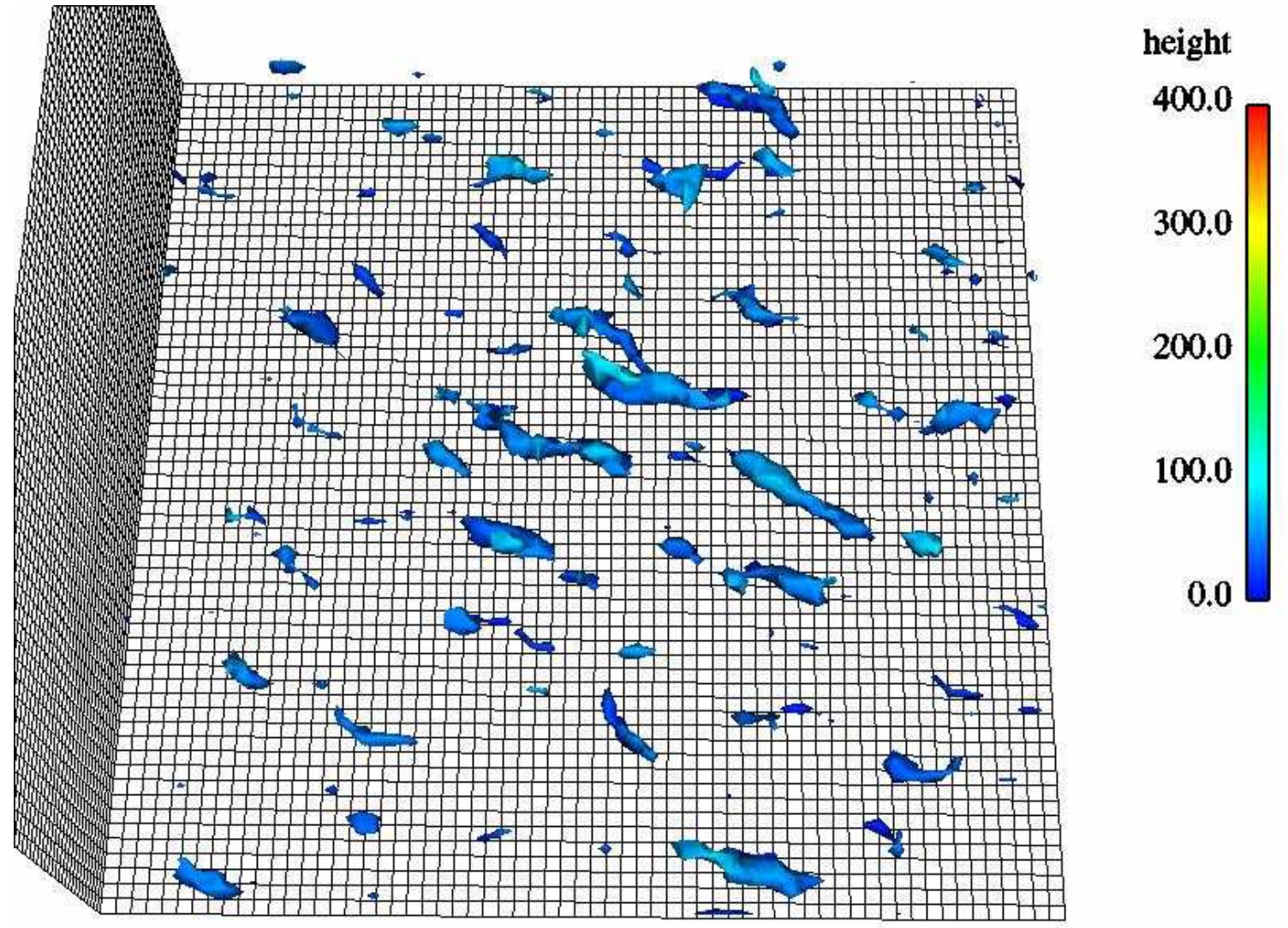

Figure 7.19: Isosurfaces of $\lambda_{2}=-0.0005$ colored by height for Run G5wt-02 at hour 8; flow is from bottom to top; horizontal axes are south-north (right-left: $0-960 \mathrm{~m}$ ) and west-east (bottom-top: $0-960 \mathrm{~m}$ ). 


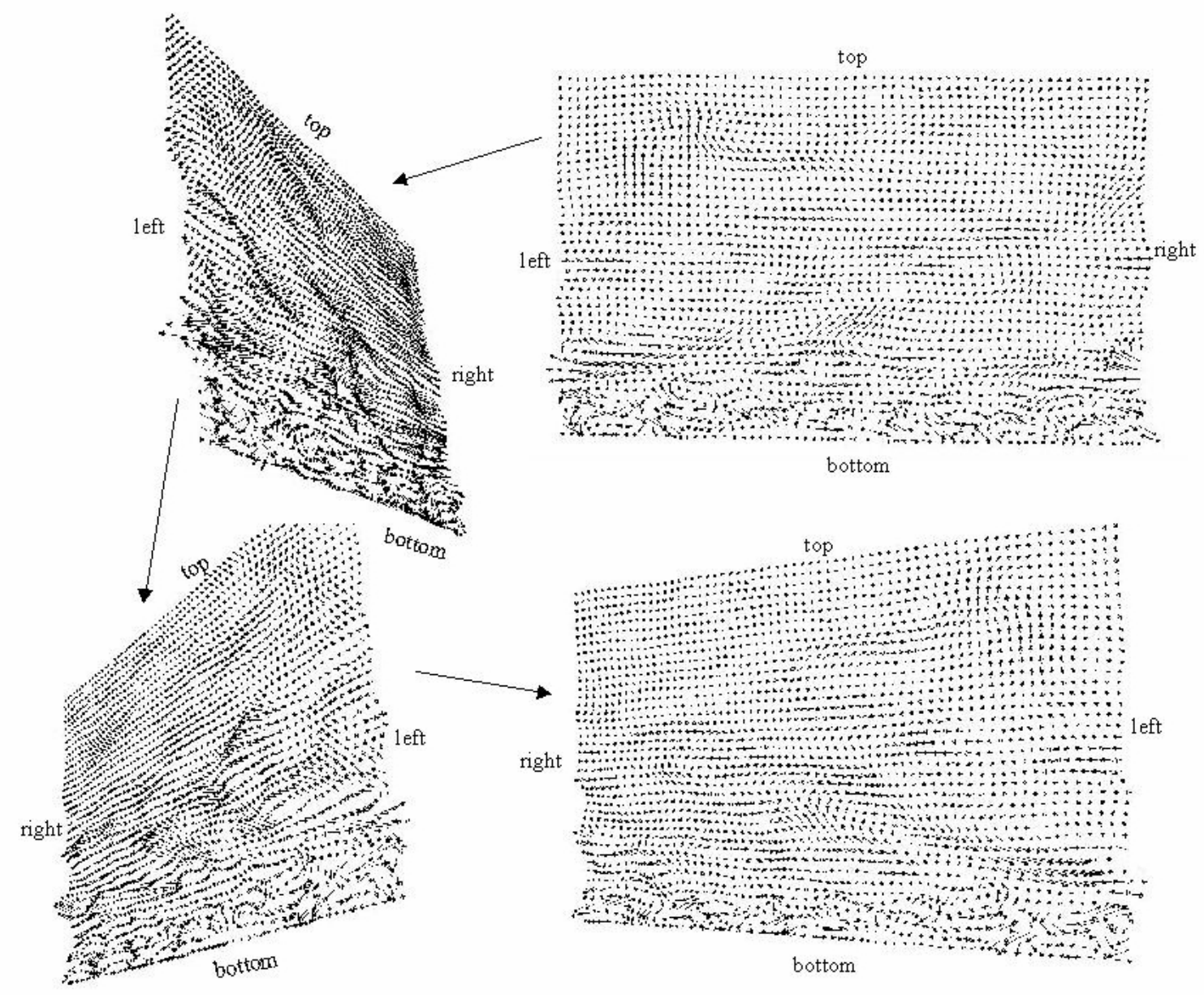

Figure 7.20: Four perspectives of the fluctuating velocity vectors at $y=300 \mathrm{~m}$ for Run G5wt-02F at hour 10; from upper right counterclockwise to bottom right, the plane is being rotated so the the left edge passes toward the viewer; plotted vectors are enhanced by a factor of 20 for visualization; horizontal axis is west-east direction $(0-320 \mathrm{~m})$; vertical axis is height $(0-200 \mathrm{~m})$. 


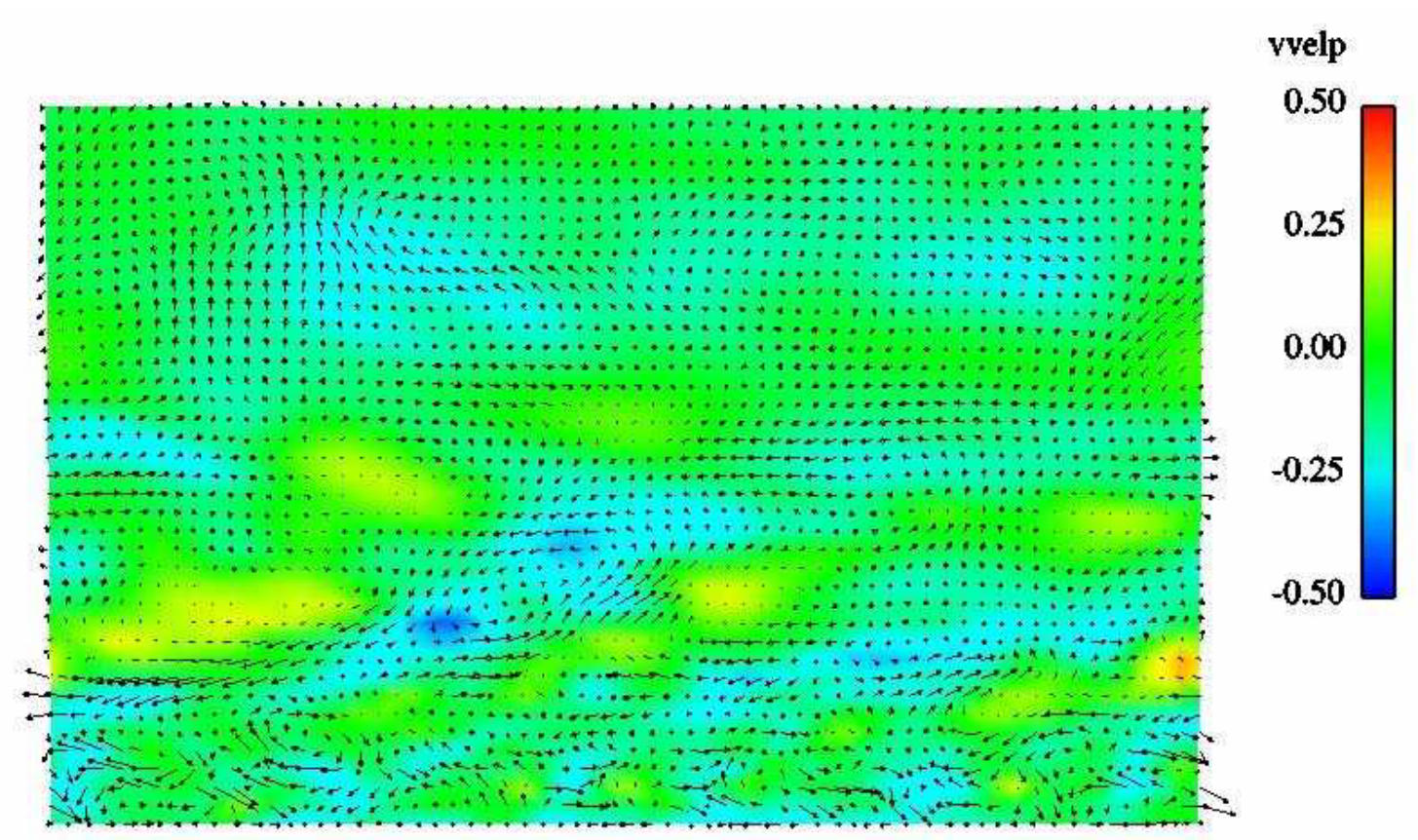

Figure 7.21: Fluctuating velocity vectors in $\mathrm{x}-\mathrm{z}$ plane at $\mathrm{y}=300 \mathrm{~m}$ for Run $G 5 w t-02 F$ at hour 10, with fluctuating velocity in the y direction $(\mathrm{m} / \mathrm{s})$ indicated by color; plotted vectors are enhanced by a factor of 20 for visualization; horizontal axis is west-east direction (0 $320 \mathrm{~m})$; vertical axis is height $(0-200 \mathrm{~m})$. 


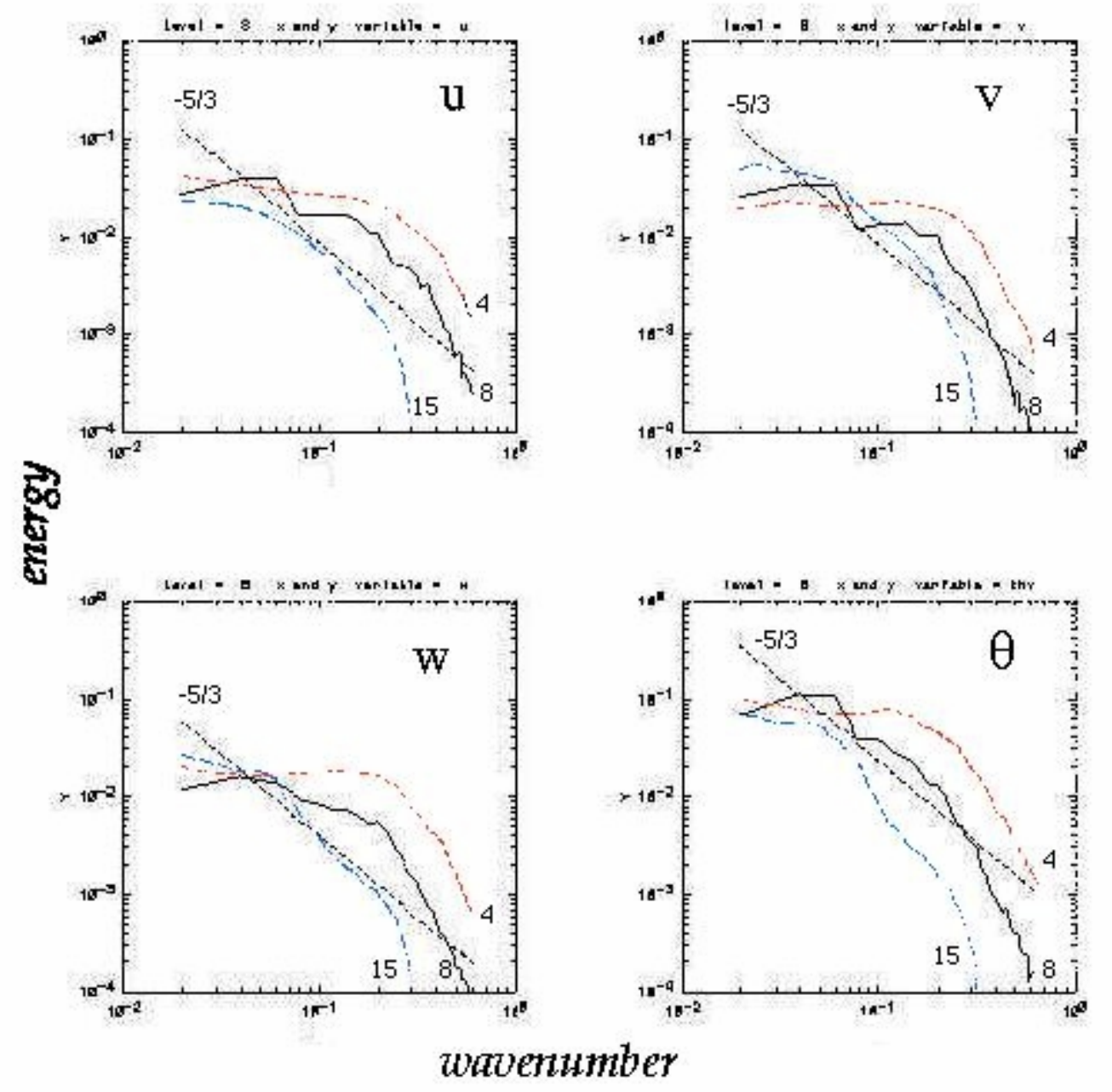

Figure 7.22: Spectra at 3 heights for Run G5wt-02F at hour 10 for variances of u, v, and $\mathrm{w}$ velocity variances and temperature variance; spectra are for three model levels: level 4 (red) $\mathrm{z}=12.5 \mathrm{~m}$, level 8 (black) $\mathrm{z}=32.5 \mathrm{~m}$, level 15 (blue) $\mathrm{z}=67.5 \mathrm{~m}$. 


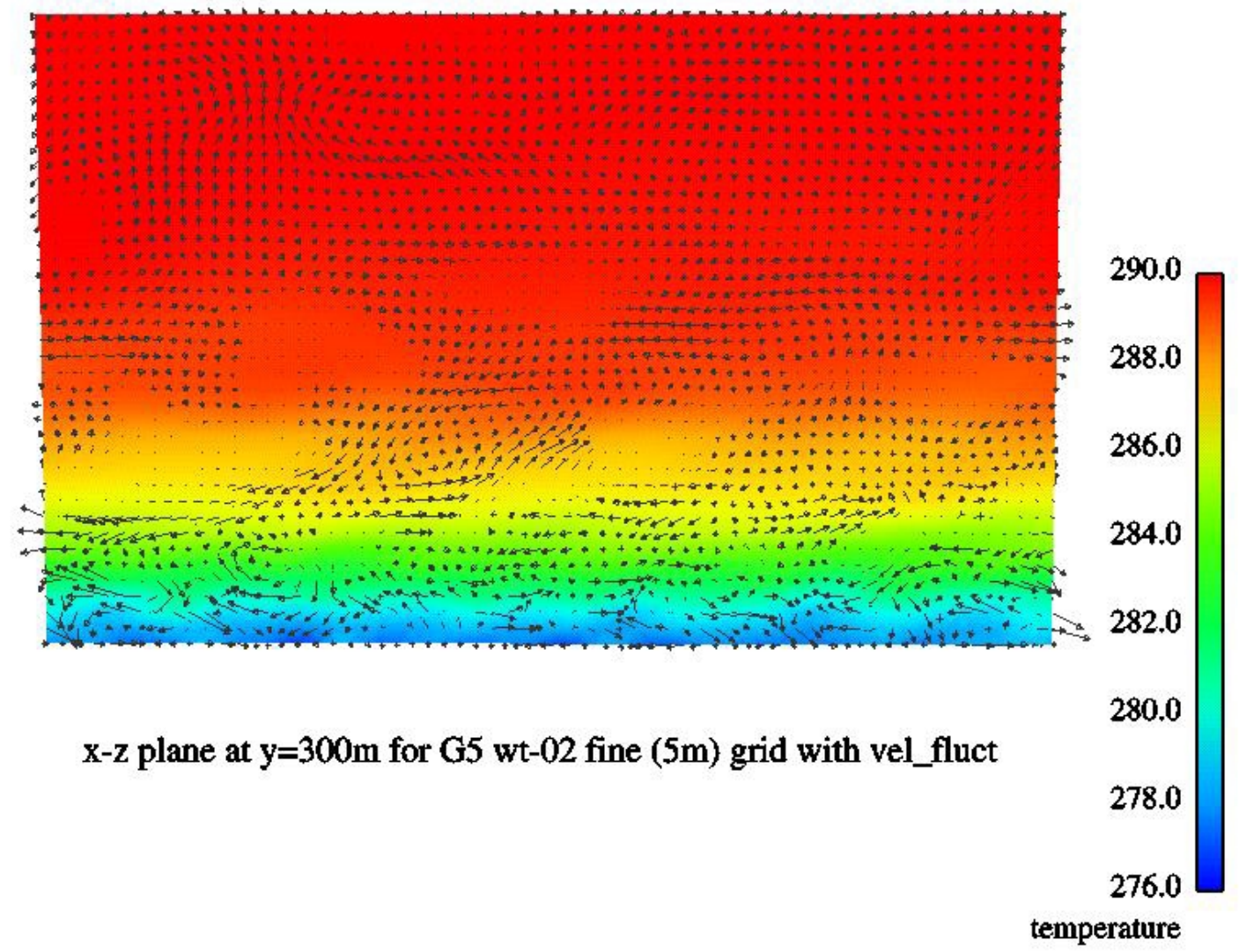

Figure 7.23: Cross section (x-z) of potential temperature with fluctuating velocity vectors at $\mathrm{y}=300 \mathrm{~m}$ for Run $G 7.5 \mathrm{wt}-02 \mathrm{~F}$ at hour 10 ; plotted vectors are enhanced by a factor of 20 for visualization; horizontal axis is west-east direction $(0-320 \mathrm{~m})$; vertical axis is height $(0$ - $200 \mathrm{~m})$. 


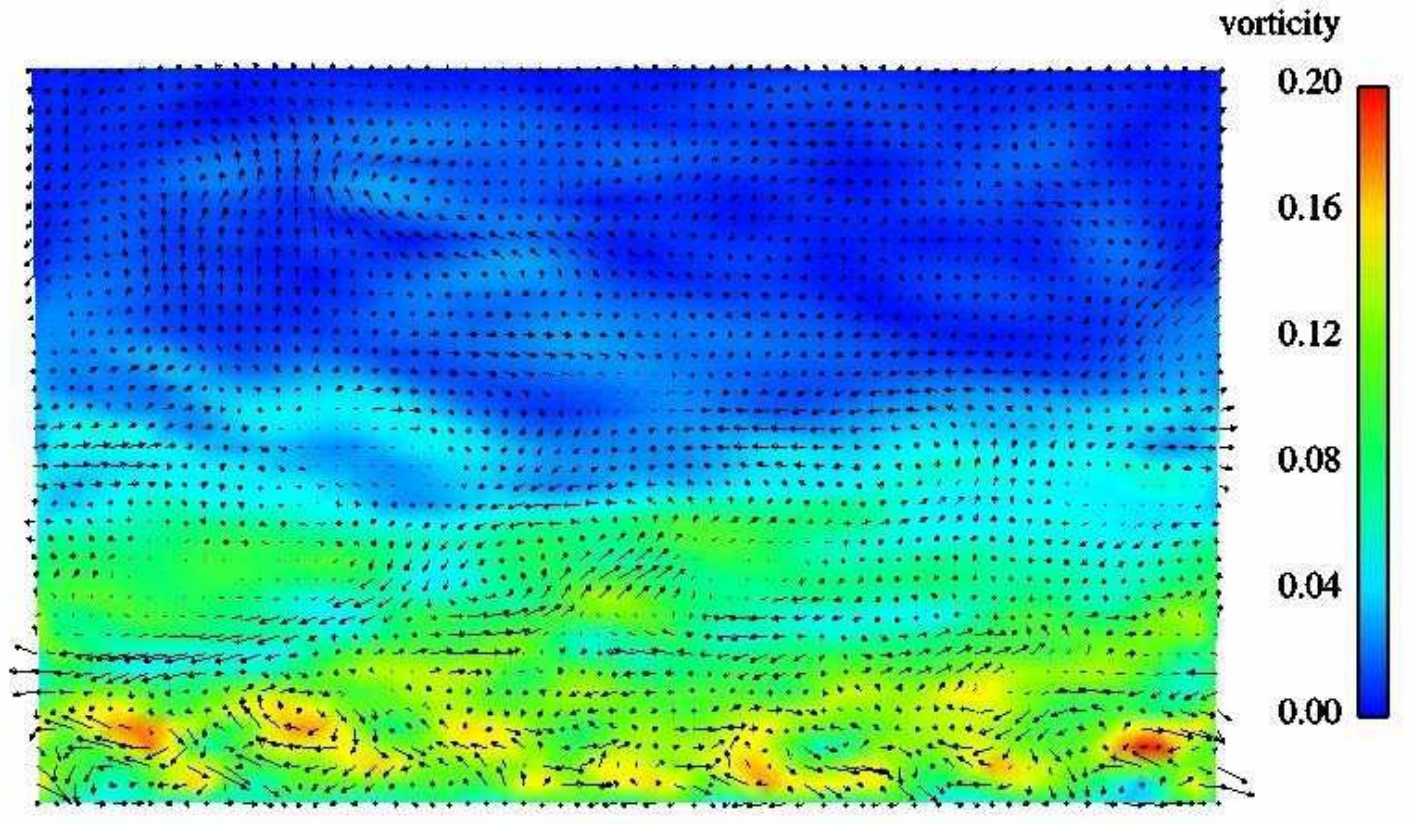

Figure 7.24: Total vorticity field at $\mathrm{y}=300 \mathrm{~m}$ for Run $G 5 w t-02 F$ at hour 10 , with fluctuating velocity in the $\mathrm{x}-\mathrm{z}$ plane superimposed; plotted vectors are enhanced by a factor of 20 for visualization; horizontal axis is west-east direction $(0-320 \mathrm{~m})$; vertical axis is height $(0-200 \mathrm{~m})$. 


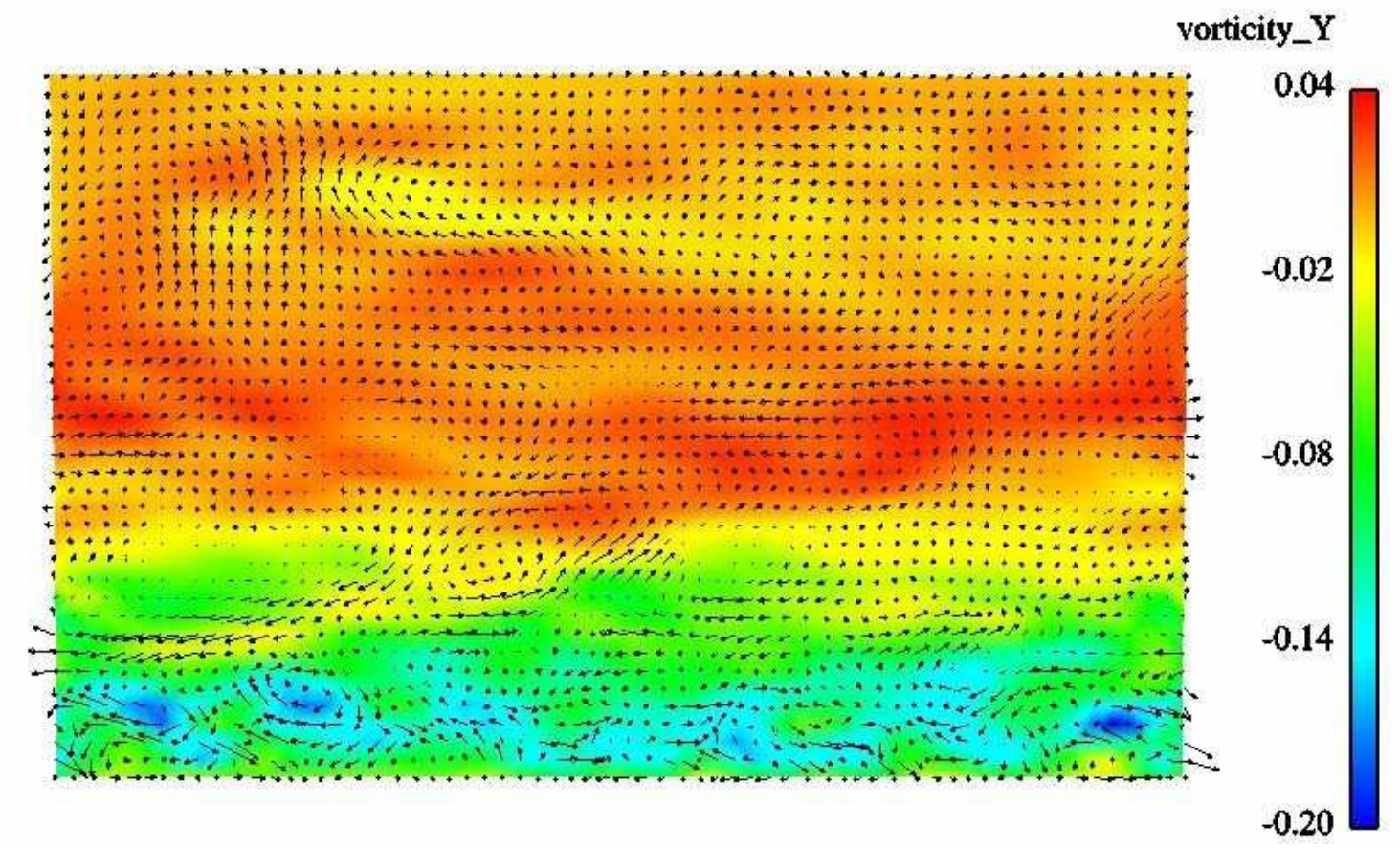

Figure 7.25: Y-component of vorticity at $\mathrm{y}=300 \mathrm{~m}$ for Run $G 5 w t-02 F$ at hour 10 , with fluctuating velocity in the $\mathrm{x}-\mathrm{z}$ plane superimposed; plotted vectors are enhanced by a factor of 20 for visualization; horizontal axis is west-east direction $(0-320 \mathrm{~m})$; vertical axis is height $(0-200 \mathrm{~m})$. 


\section{Chapter 8}

\section{Implications for Dispersion in the SBL}

One of the primary benefits of this research is an improved understanding of turbulent dispersion in the SBL. This is often the 'worst-case' scenario addressed in studies of health impacts associated with the release of hazardous materials to the atmosphere. LES of the evolving SBL provides unique data sets for assessing the effects of stable stratification on transport and dispersion. The simulations include the initial development of the CBL in the afternoon, followed by the development of an SBL after sunset with a strong, surface-based temperature inversion. The structure of the turbulence is modified significantly by negative buoyancy associated with the temperature inversion. The magnitude of velocity variances is reduced by an order of magnitude compared to that in the CBL, and the vertical velocity variance is damped further as the static stability preferentially damps vertical motions. The advanced SGS turbulence model allows simulation of intermittently enhanced periods of turbulence in the SBL that are often observed. During these turbulent episodes, mixing is enhanced throughout the SBL. Air pollution models that account only for the long-term mean structure of the SBL do not include the effects of these episodes.

LES results can be used as surrogate wind fields in which marker particles can be tracked to illustrate the transport and dispersion of material. The LES results from this research imply that material released near the surface and mixed to higher elevations would be transported by stronger winds and in different directions, because of the vertical shear of horizontal wind speed and direction. Material released at altitude in the SBL will tend to be mixed down toward the surface during these turbulent episodes in a fumigation-like scenario at night. 


\subsection{The Approach and LES Results Used}

\subsubsection{Source of simulated wind fields}

The LES-generated wind fields are from a sensitivity run $(G 10.4 w t-02 T)$ with a smaller domain: $640 \mathrm{~m}$ in each horizontal direction, and $400 \mathrm{~m}$ in the vertical direction. The numerical grid is oriented west-east for the x-direction (the nominal surface wind direction), and south-north for the y-direction. The grid resolution for the simulations is $20 \mathrm{~m}$ in the horizontal directions and $5 \mathrm{~m}$ in the vertical direction. Although the depth of the simulated CBL is smaller than typically observed, the simulated turbulence structure scales with observations, and provides the desired afternoon conditions prior to the evening transition. The resulting SBL is similar to observations.

Vertical profiles of temperature and winds for the simulated CBL agree well with observations with small vertical gradients below the capping inversion. This is due to the large amount of turbulence, especially in the vertical direction, within the mixed layer below the capping temperature inversion. The simulated turbulence structure is dominated by the vertical velocity variance within the mixed layer, while the horizontal velocity variances dominate near the ground. After 5.5 hours of cooling, the evolving SBL develops the expected strong, surface-based temperature inversion, and the associated strong wind shear as shown in Figure 8.1. In contrast to the $\mathrm{CBL}$, the vertical velocity variance is a minimum in the SBL, and the turbulence is primarily in the horizontal velocity fluctuations and is a maximum near the ground where generation by wind shear is the greatest.

The vertically-integrated velocity variances provide a clear picture of the evolution of turbulence after sunset. As can be seen in Figure 8.2, the collapse of the mixed layer and its associated large amount of turbulence occurs rather completely during the 1-hour transition period of surface heating to surface cooling. The turbulence is not quenched during the continued surface cooling. Instead, there are temporally varying levels of turbulence throughout the night. As noted in Figure 8.2, there is an event of enhanced turbulence after 6 hours of surface cooling. The vertical profiles of the resolved velocity variance before and during this event show the deepening of turbulence activity throughout the SBL, especially in the horizontal velocity components 
(see Figure 8.3). After the event, the turbulence is greatly reduced in all components, and is confined mainly to a shallow layer near the ground. Spectral analysis of the velocity components shows that the enhanced turbulence occurs at all scales, but has the effect of moving the spectral peak toward smaller scales. In contrast, the strong reduction of turbulence after the event takes place more in the smaller scales, and spectral peak occurs at scales larger than those before the event.

\subsubsection{Marker particles in simulated wind fields}

The effects of intermittently enhanced turbulence on transport and dispersion in the SBL are evaluated by releasing marker particles in the LES-generated wind fields, following a technique used by Kemp and Thomson (1996). In the present study, the SGS turbulence is not incorporated since it is a very small fraction of the total turbulence, being an important fraction only near the ground where simulated releases are not made. If the SGS turbulence were to be included in the particle transport velocities, it would be through random perturbations scaled by the SGS TKE. Wang and Squires (1996) conducted an investigation of the effect of the SGS velocity field on the particle transport by such an approach, and found that, at most, the difference as less than $1 \%$.

The release scenario is a collection of 75 marker particles released every second and tracked to illustrate the different transport and dispersion features of the simulated flows. The release positions are centered on a specified release point, with 5 positions in each of the horizontal directions equally spaced across $10 \mathrm{~m}$, and repeated 3 times in the vertical direction, equally spaced across $1 \mathrm{~m}$. The release duration is 5 minutes, giving a population of 22,500 particles for each release. After some time, particles are transported horizontally beyond the model grid. The use of periodic boundary conditions allows the effective wind field domain to be expanded by adding the computed domain to any adjacent side as needed (like tiles on a floor), as was done by Kemp and Thomson (1996).

The greatest variation of transport and dispersion factors in the SBL occurs in the vertical direction, due to the strong wind shear and the variation in strength of turbulence. For this reason, five release heights are specified $(10 \mathrm{~m}, 30 \mathrm{~m}, 50 \mathrm{~m}, 70 \mathrm{~m}$, 
and $90 \mathrm{~m}$ ) at the same horizontal location to study the differences in transport and dispersion. For purposes of comparison, marker particles are also released within the CBL, at just two heights (20m and $60 \mathrm{~m})$.

\subsection{Simulated Dispersing Plumes of Material}

The collection of marker particles at a given time after the continuous release of particles forms plume-like patterns. To illustrate this, trajectories of emitted particles for Run G5wt-02F at hour 10 are shown in Figure 8.4. Releases are made at 7 evenly spaced heights between $10 \mathrm{~m}$ and $130 \mathrm{~m}$, and represent 50 seconds of continuous release. Spheres of expanding size are used to mark the location of the particles to illustrate plume spread. The effect the strong directional shear is demonstrated by the shifting direction of plume transport as release points increase with height up to a point where the winds become fairly constant. Also, the low wind speed near the surface is evident in the reduced distance of transport. In the figures that follow, two views are given for each scenario to capture the plume transport: (1) in the x-z plane, i.e. looking north, and (2) in the $\mathrm{x}-\mathrm{y}$ plane, i.e. looking down.

The locations of marker particles after 5 minutes for Run G10.4wt-02T reveal plume-like patterns for each release height, as shown in Figure 8.5 for the simulated wind fields before the enhanced turbulence event. Note that the vertical scale is exaggerated to facilitate visualization. The height dependence of speed and direction of transport is clearly evident. There is not a strong variation in dispersion with height.

The locations of marker particles released during the enhanced turbulence event tell a different story. Within the middle portion of the SBL, there is much greater dispersion, as seen in Figure 8.6. This leads to significantly reduced concentration of released material. The increased velocity variance shown in Figure 8.3b accounts for the increased turbulent dispersion illustrated in the simulated plumes. This is an important phenomenon in the SBL that traditional air pollution models treating only long-term averages will miss. The periods of enhanced turbulence in the LES-generated atmosphere lead to larger plume volumes. Although this will result 
in reduced concentrations, it can also result in the transport of material at still hazardous levels over larger volumes. For example, a plume of hazardous material could be transported aloft for some distance with minimal dispersion and then encounter enhanced turbulence that would mix the plume down to the ground at hazardous concentrations.

For comparison, marker particles were released in a simulated CBL and produced a dramatically different result than for the SBL. The vertical transport by large convective eddies is seen in Figure 8.7a. The small vertical gradient of wind direction within the well-mixed CBL is evident in Figure 8.7b, when compared to that in the SBL (see Figures 8.5b and 8.6b). The CBL is a much different environment for transport and dispersion, and this is captured in the LES-generated wind fields. A release aloft can be transported down to the ground close to the source with still fairly high concentrations. However, once the plume begins to move downwind, the vigorous mixing quickly reduces the concentration, much more quickly than in the SBL.

\subsection{Estimates of Eddy Diffusivity}

LES results can be used profitably to estimate parameters that are of practical value in dispersion applications. Of great interest in dispersion models is the eddy diffusivity $K_{c}$ which allows one to calculate turbulent fluxes of contaminants using known spatial gradients of mean concentration. For example, the vertical flux of concentration $\mathrm{C}$ is modeled as

$$
\overline{w^{\prime} C^{\prime}}(z)=-K_{c}(z) \frac{\partial C}{\partial z}
$$

The eddy diffusivity is basically the ratio flux / gradient. The choice of how to express the flux and gradient results in numerous prescriptions for eddy diffusivity which use of a range of parameters that describe the turbulent state of the flow.

For the results that follow, potential temperature will be used as the scalar quantity (in place of concentration), since mean potential temperature fields and turbulent heat fluxes are available from the LES results. The eddy diffusivity that relates the 
vertical flux of potential temperature to the vertical distribution of potential temperature is:

$$
K_{\theta}(z)=-\frac{\overline{w^{\prime} \theta^{\prime}}(z)}{(\partial \theta / \partial z)}
$$

The quantities on the RHS of Equation 8.2 are readily available from the simulations conducted. For example, the eddy diffusivity profiles for the 10-minute periods before, during, and after the turbulent episode described in Section 6.4 are calculated using Equation 8.2, and plotted in Figure 8.8. The effect of the episode is evident in the increased values of eddy diffusivity up in the SBL where the enhanced turbulence occurs. This increase is consistent with the increase in heat flux during the episode, shown in Figure 6.28.

The eddy diffusivity can be modeled in terms of the flux Richardson number $R_{f}$ (see Equation 7.3) and the dissipation rate $\varepsilon$ (see Equation 7.1), following Osborn (1980)

$$
K_{\theta}(z)=\left(\frac{R_{f}}{1-R_{f}}\right) \frac{\varepsilon}{N^{2}}
$$

Using the values for the turbulent episode as done above, vertical profiles of $K_{\theta}$ can be estimated (see Figure 8.9). As with use of Equation 8.2, the enhanced turbulence during the episode is reflected in corresponding increase in eddy diffusivity, obtained from Equation 8.3. There are minor differences, however; the eddy diffusivity from Equation 8.3 has smaller values near the ground and asymptotes to the small values aloft at a lower height than values obtained from Equation 8.2. The different behavior in the eddy diffusivity profile from Equation 8.3 is caused by the vertial distribution of dissipation, which is small near the surface and decreases to minimum values aloft at a lower height (see Figure 6.30) than does the heat flux used in Equation 8.2.

The vertical distributions of parameter values describing turbulence in the flow are often not available in practical applications. However, values are usually available near the surface from single point measurements. Practical models of eddy diffusivity must then parameterize the vertical variation of the eddy diffusivity, based on a basic prescription for the eddy diffusivity and some height-dependent function. One such model is (Lange, 1989):

$$
K_{\theta}(z)=\frac{u_{*} k z}{\phi_{h}(z / L)} e^{-c z / h}
$$


where $\mathrm{h}$ is the depth of the boundary layer and $\mathrm{c}$ is a constant set to 4 in typical implementations. The value of $\phi_{h}$ for the SBL is $\phi_{h}=1+5 z / L$ (Dyer, 1974). L is the Monin-Obukhov length, defined in terms the surface stress and buoyancy flux:

$$
L=\frac{-u_{*}^{3}}{k(g / \theta) \overline{w^{\prime} \theta^{\prime}}}
$$

For the turbulence episode, the depth of the SBL is 190m at hour 11 (see Figure 6.24). For the periods before, during, and after the turbulent episode, the vertical profiles of eddy diffusivity obtained from Equation 8.4 are nearly the same, with some small differences near the ground. This is not unexpected, since Equation 8.4 is sensitive only to near-surface stress and heat flux which are affected very little by the turbulent episode.

If vertical distributions of turbulence parameters are available, then Equation 8.4 can be recast in terms of local scaling or 'z-less' scaling, as appropriate (see Subsection 2.1.4 and Figure 2.16). Also, since vertical information about the turbulence is now included, the height-dependence function $\left(e^{c z / h}\right)$ is eliminated. For local scaling, the local Monin-Obukhov length (now denoted by $\Lambda$ ) is defined by Equation 8.5, except that the surface stress and heat flux are replaced by local stress and heat flux. Local scaling is appropriate for weak to moderate stability $(z / \Lambda<1)$. The only difference in Equation 8.4 for local scaling is that $\phi_{h}$ is evaluated using $z / \Lambda$ instead of $z / L$ in Equation 8.5. When stability becomes stronger, vertical motions are reduced to the point where the effect of the ground vanishes and there is no longer a dependence on height (i.e. the similarity is 'z-less'). The only length scale is $\Lambda$. In 'z-less' scaling, one goes a step further in modifying Equation 8.4 by replacing the length scale $k z$ with $\Lambda$. The resulting eddy diffusivity profile for the period before the episode is very noisy, with $K_{\theta}$ values exceeding a value of 7 in the upper part of the SBL, as seen in Figure 8.10. An eddy diffusivity profile from Equation 8.4, using the usual surface scaling, is included as a reference in the figure. The shape of the profile during the episode looks reasonable and reflects the enhanced turbulence. However, the magnitude of the eddy diffusivity up in the SBL is about twice that from Equations 8.2 and 8.3; note that the horizontal axis values are doubled in the figure compared to those in the previous two figures. 
If the length scale used to replace $k z$ in Equation 8.4 is $u_{*}^{\text {local }} / N$ (Nieuwstadt, 1985) instead of $\Lambda$, and if that length scale is used instead of $\Lambda$ in the evaluation of $\phi_{h}$, then better profiles are obtained (see Figure 8.11). The eddy diffusivity profile during the episode agrees qualitatively with those obtained from Equations 8.2 and 8.3. This can be seen when the vertical profiles for all approaches are compared for the 10-minute period during the episode (see Figure 8.12). Clearly all but the standard surfacebased model (Equation 8.4) reflect the enhanced turbulence up in the SBL during the episode. If estimates from Equation 8.2 are taken to be the most accurate, since they are most directly related to the basic definition of eddy diffusivity, then the results from Equation 8.4 with local scaling compare best in the lower SBL below a height of $50 \mathrm{~m}$, while those from Equation 8.3 compare best above that. Interestingly, the lower SBL where estimates from Equations 8.2 and Equation 8.4 with local scaling compare well is the same region where mixing and stirring are rather unaffected by the episode (see Section 7.3) As mentioned before, the surface-based approach (Equation 8.4) does not include the effects of any episodic turbulence away from the surface, and thus compares poorly at heights between $20 \mathrm{~m}$ and the top of the SBL during the episode.

To complete the comparison of the various estimates of eddy diffusivity, profiles obtained for the 10-minute periods before and after the episode are shown in Figures 8.13 and 8.14, respectively. During these periods, all approaches have eddy diffusivities that decrease with height in the lower SBL. Again taking the eddy diffusivity estimates from Equation 8.2 as most accurate, the results from Equations 8.3 and 8.4 (with local scaling) bracket the desired values on the low and high side, respectively. Interestingly, the standard surface-based model (Equation 8.4) does a good job of representing the eddy diffusivity profile. Thus, for periods in the SBL when the turbulence is not enhanced episodically, the eddy diffusivity approach often used in practical applications performs surprisingly well.

The nondimensional temperature gradient $\phi_{h}$ is used in Equation 8.4. In the SBL, it is very similar to or the same as the nondimensional wind shear $\phi_{m}=1+z / L$. Values for $\phi_{m}$ are obtained using LES-generated wind profiles from Run G7.5wt-02 over a 1-hour period (hour 13 to 14) and the definition of $\phi_{m}$ (see Equation 5.33). The resulting vertical profile is shown Figure 8.15, along with the curve for $\phi_{m}=1+z / L$. In the lower part of the SBL, the LES-generated profile and the Dyer relation agree 
quite well. Above a height of $40 \mathrm{~m}$, the agreement worsens, with a complete departure between profiles above a height of $70 \mathrm{~m}$. If the value of $u_{*}$ in Equation 5.33 is evaluated with the local stress rather than the surface stress, then the agreement between $\phi_{m}$ profiles obtained from LES winds and the Dyer relation is much better (see Figure 8.16).

\subsection{Discussion}

Large-eddy simulation provides realizations of atmospheric flows that are representative of both stable and unstable conditions typically observed. The simulated wind fields can be used to study transport and dispersion under a variety of atmospheric conditions, without the need to make assumptions about the turbulence, which is explicitly resolved by the LES. The SBL is of particular interest due to its frequent use as a worst-case scenario in hazard assessment, and yet our understanding of turbulence in stably-stratified conditions is incomplete. LES has the capability of providing 'numerical' experiments to increase our understanding. In addition, LES results can allow us to study transport and dispersion in the SBL, and evaluate the performance of practical dispersion models in situations where field data are not available.

The study presented here shows the ability of LES, with advanced SGS turbulence models that include energy backscatter, to capture the unsteady and intermittent behavior of turbulence in the SBL. Estimates of eddy diffusivity made from the LES results reflect the unsteady character of the eddy diffusion in the SBL during periods of enhanced turbulence. Simple, practical algorithms for eddy diffusivity cannot capture this unsteady behavior, but they are shown to represent eddy diffusion in the undisturbed SBL.

The strong wind shear in the SBL is demonstrated to have a significant, heightdependent effect on the speed and direction of transport. The enhanced periods of turbulence have a further effect on the dispersion of material, as illustrated by the location of marker particles. The enhanced dispersion leads to differential transport, especially for dispersion in the vertical direction. The resulting differences in concentrations of released material has important implications for health assessments of potential releases of hazardous materials. 


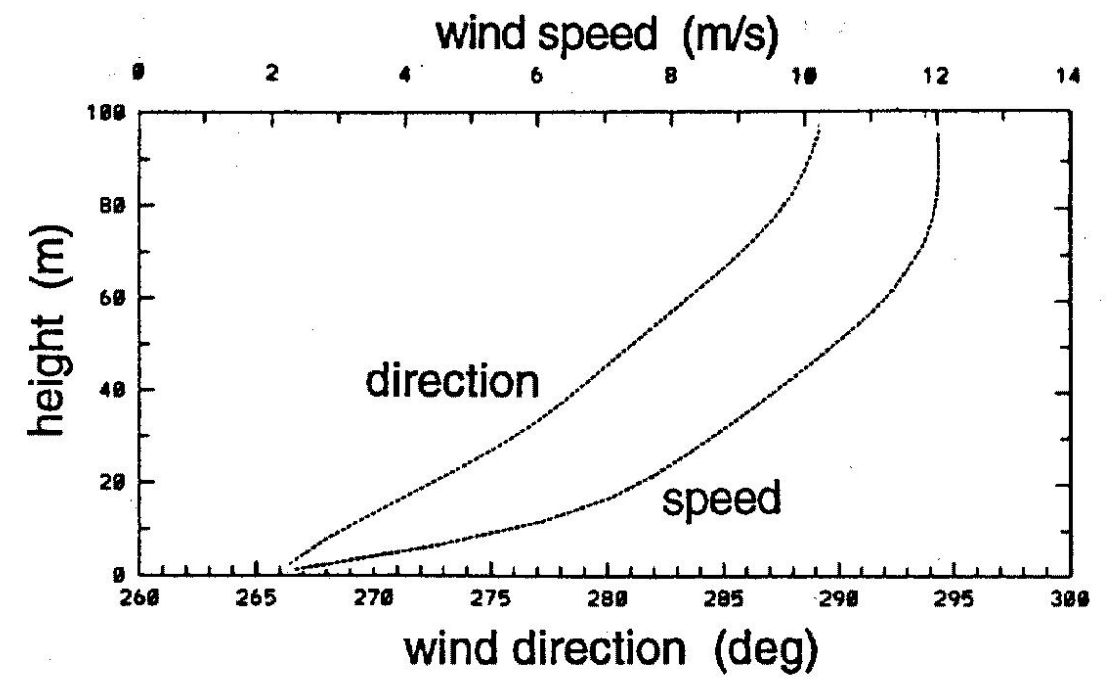

Figure 8.1: Vertical profile of mean wind speed and direction in SBL used for plume study. 


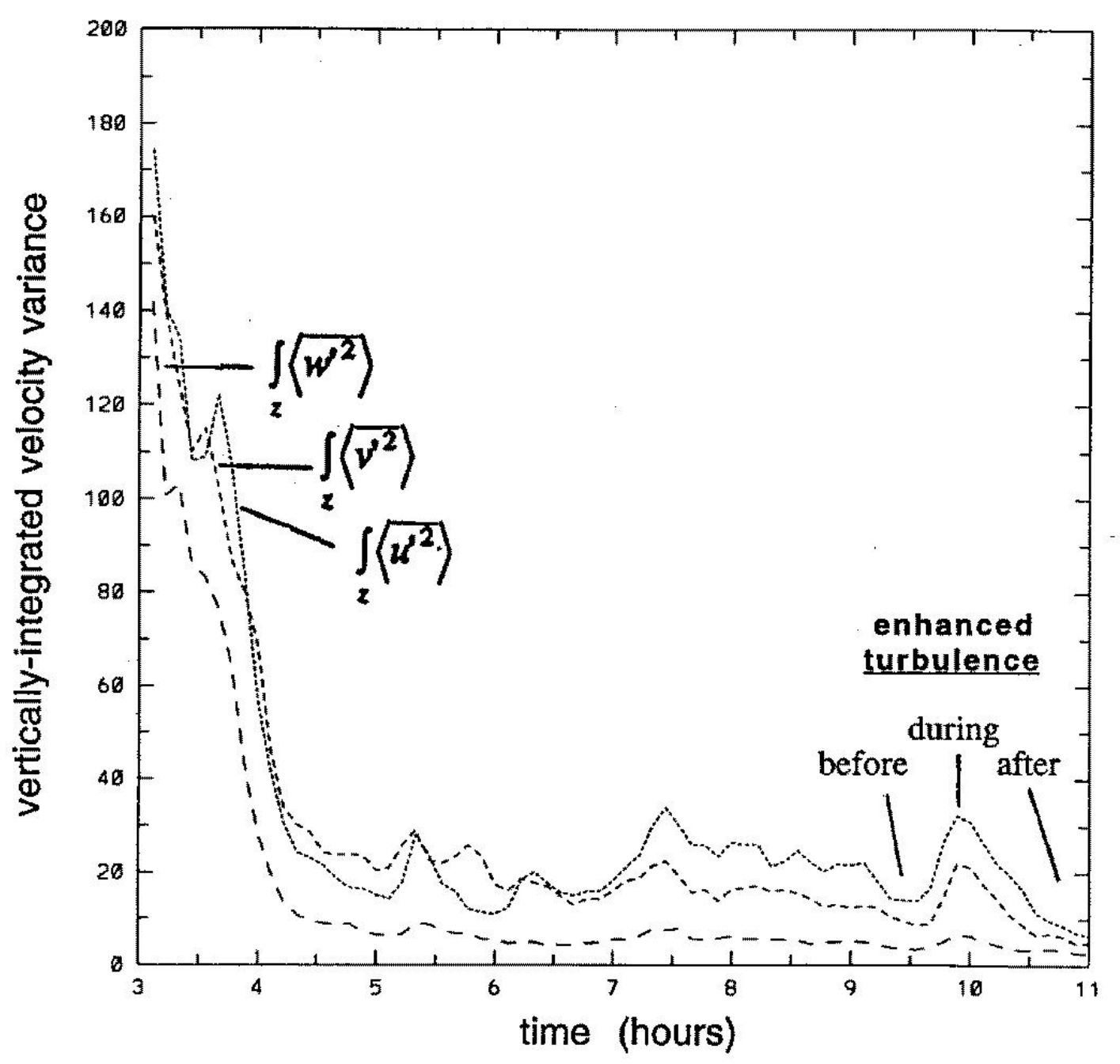

Figure 8.2: Time history of vertically-integrated velocity variances for LES generated wind fields. 

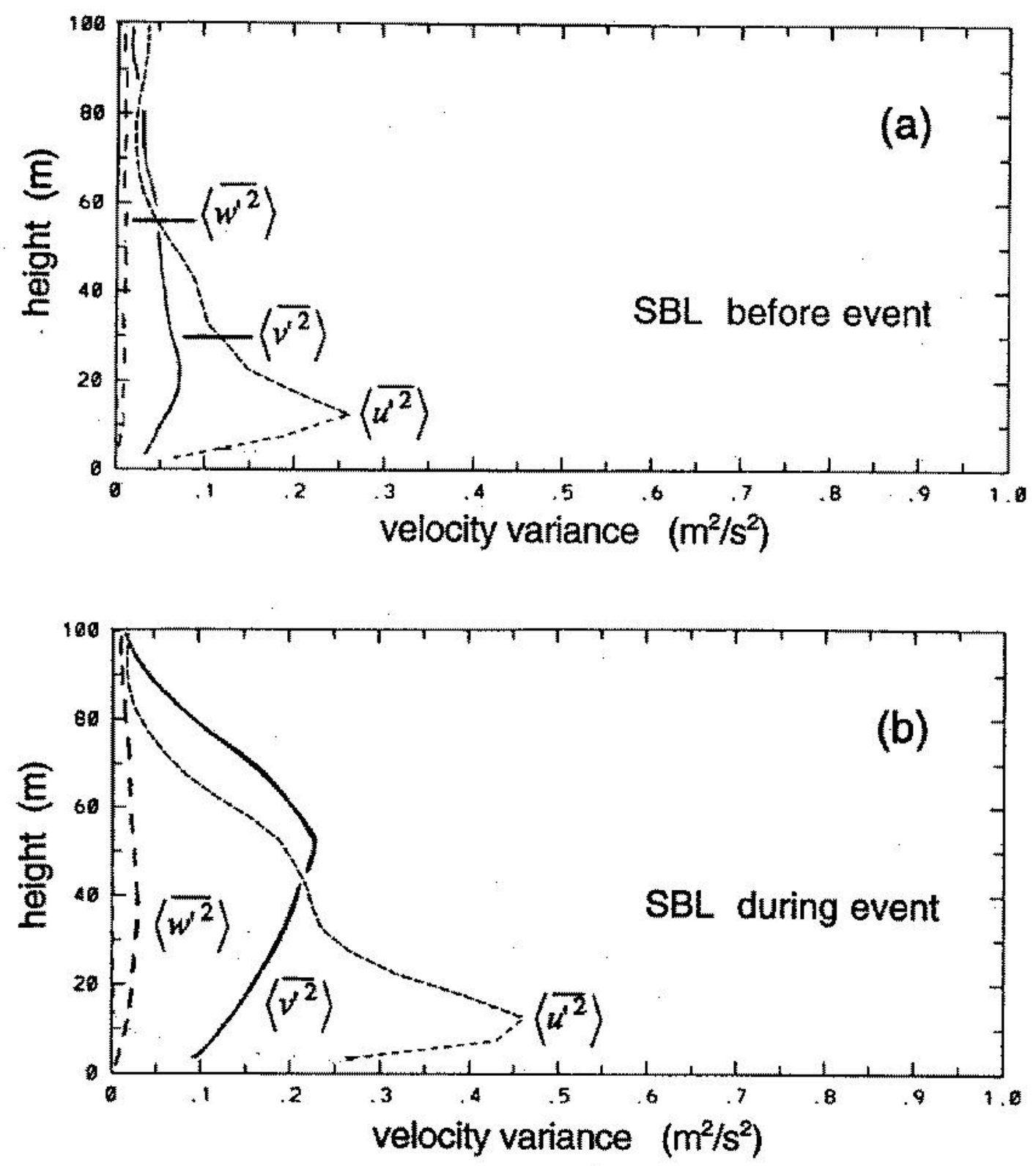

Figure 8.3: Vertical profiles of velocity variance (a) before and (b) during the enhanced turbulence event in SBL. 

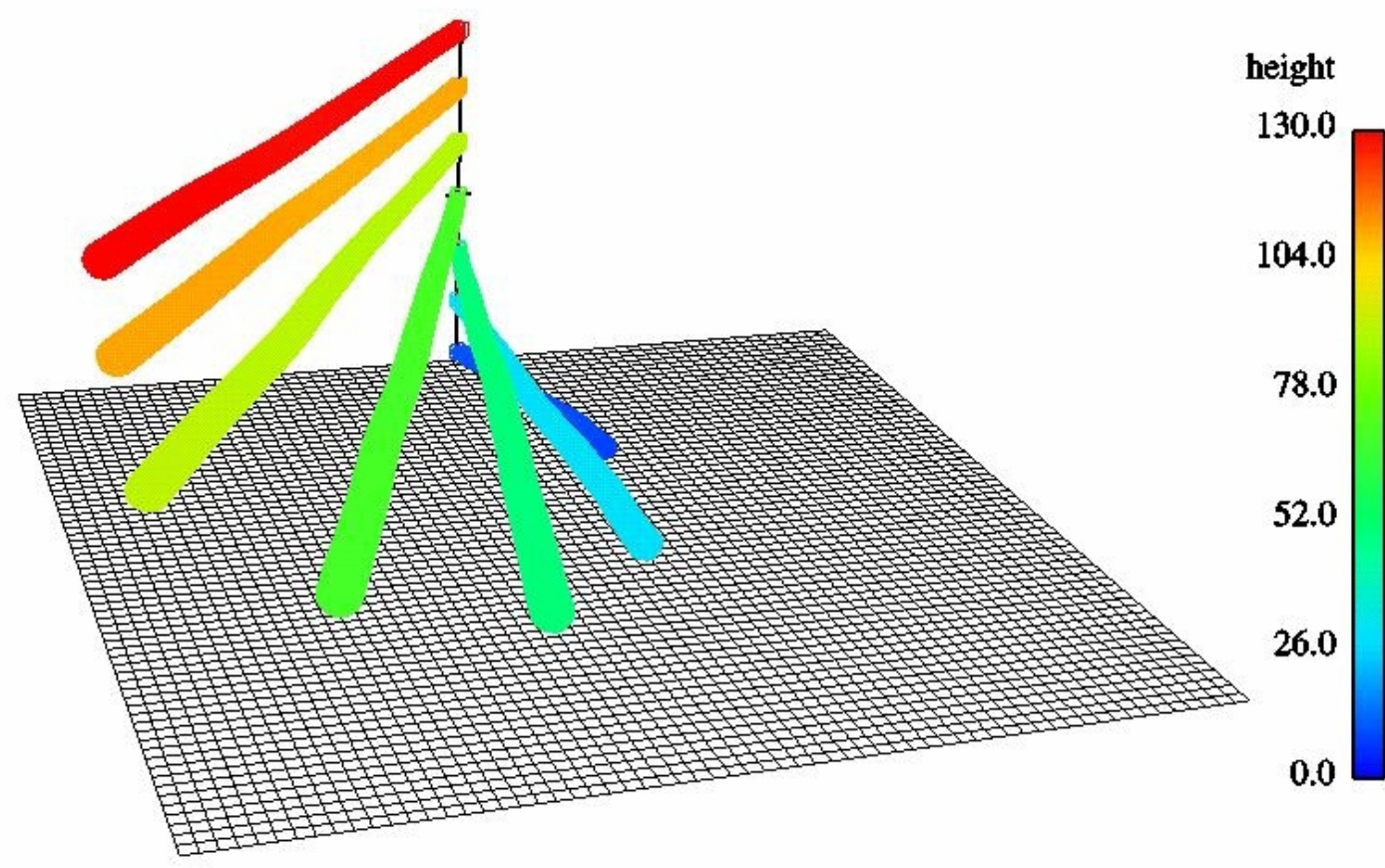

Figure 8.4: Example of idealized plumes in SBL for Run G5wt-02F; color of plume indicates height. 

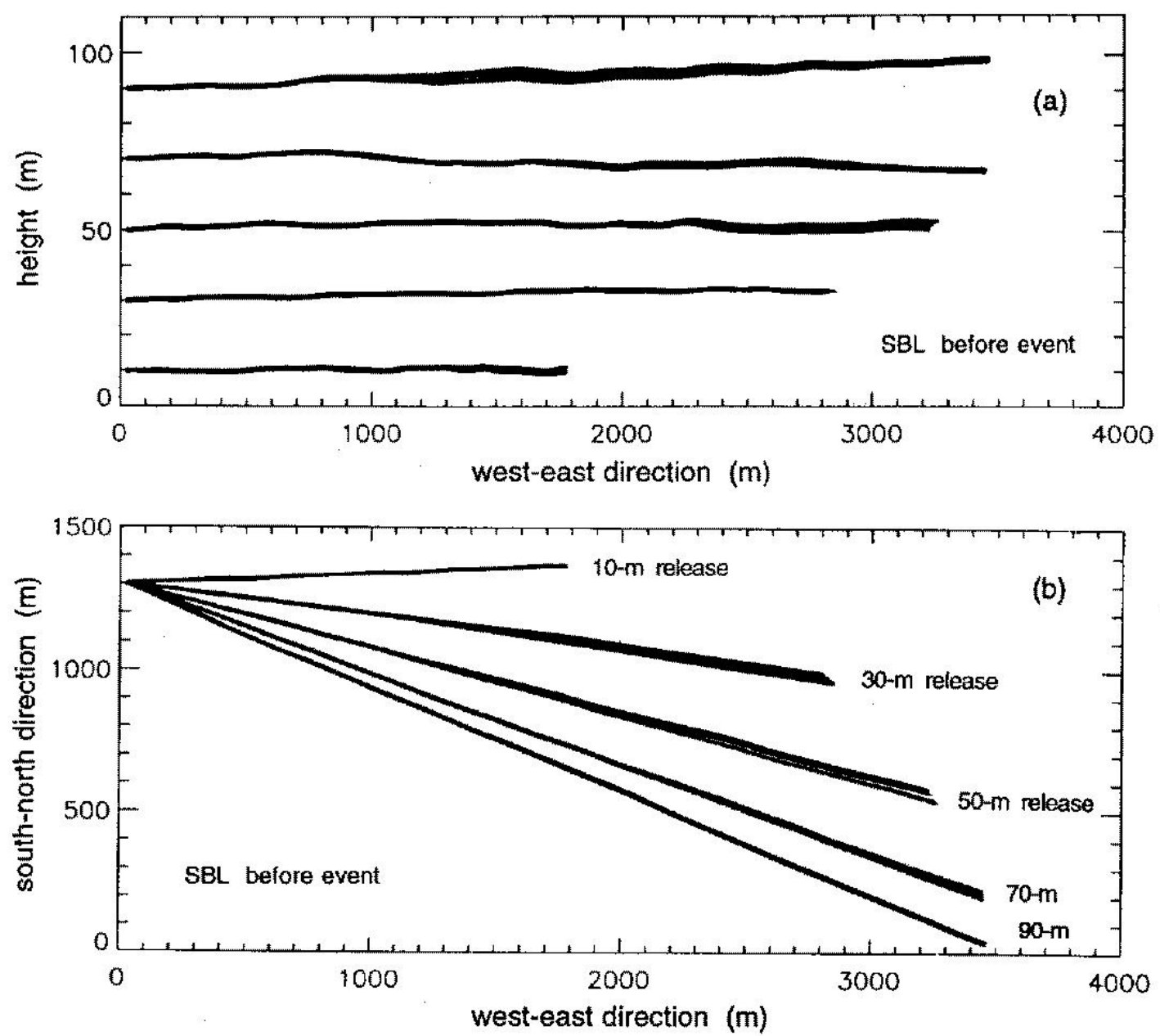

Figure 8.5: Locations of marker particles for 5-minute release into SBL for Run $610.4 w t$ 02 $T$ before turbulence event shown in (a) x-z plane, and (b) $x-y$ plane. 

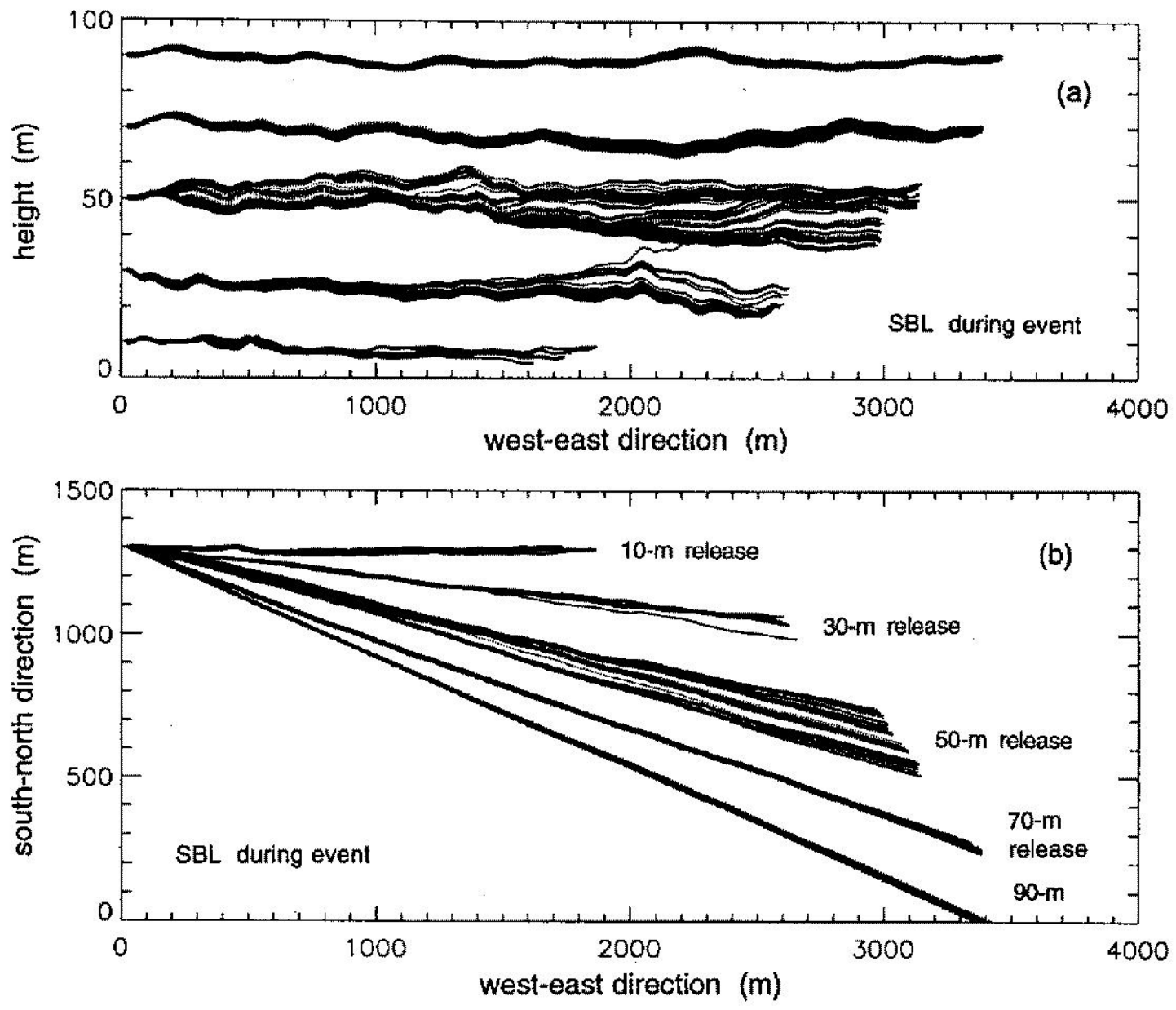

Figure 8.6: Locations of marker particles for 5-minute release into SBL for Run $610.4 w t$ 02T during turbulence event shown in (a) x-z plane, and (b) x-y plane. 

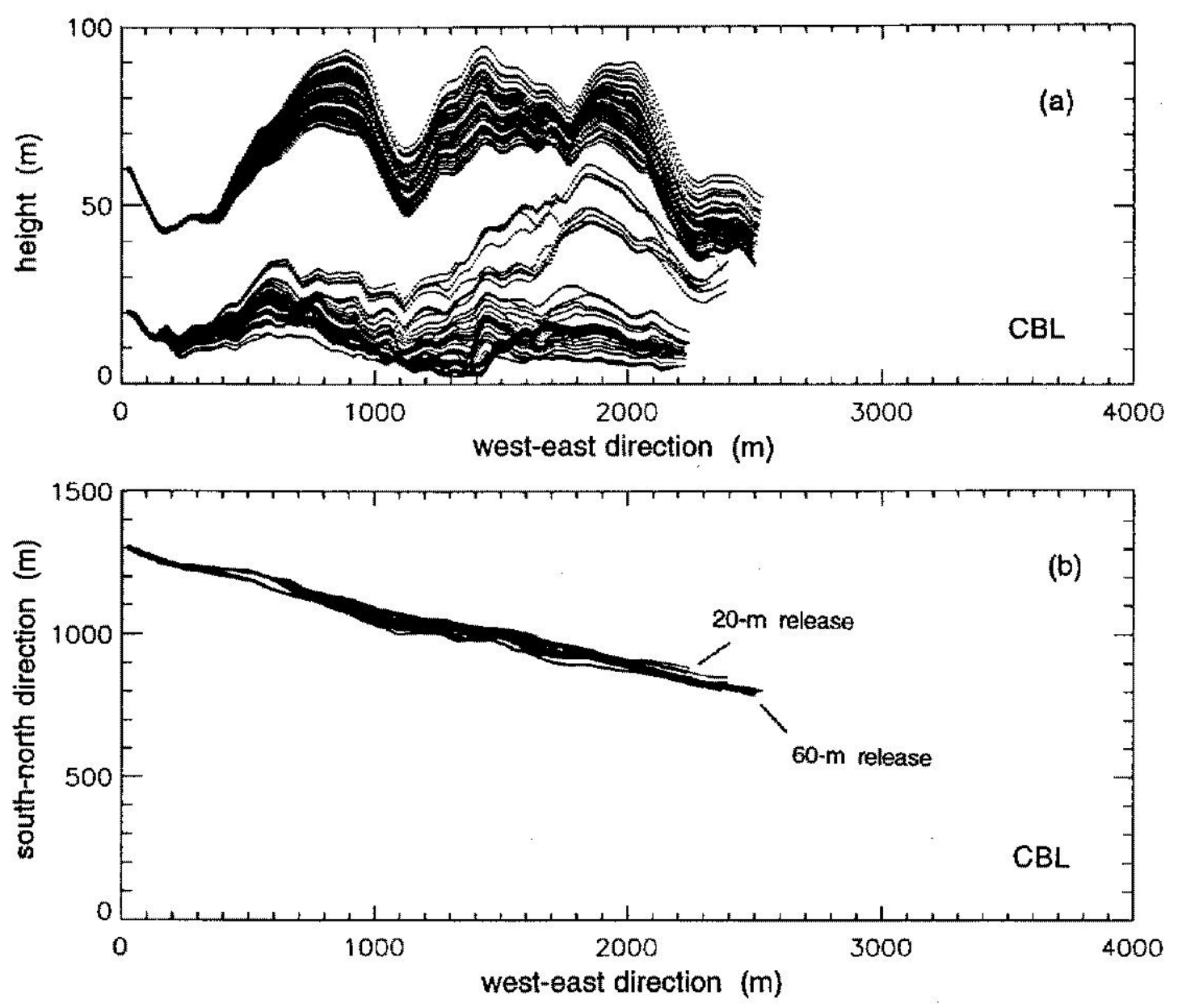

Figure 8.7: Locations of marker particles for 5-minute release into CBL for Run $610.4 w t$ $02 T$ shown in (a) x-z plane, and (b) x-y plane. 


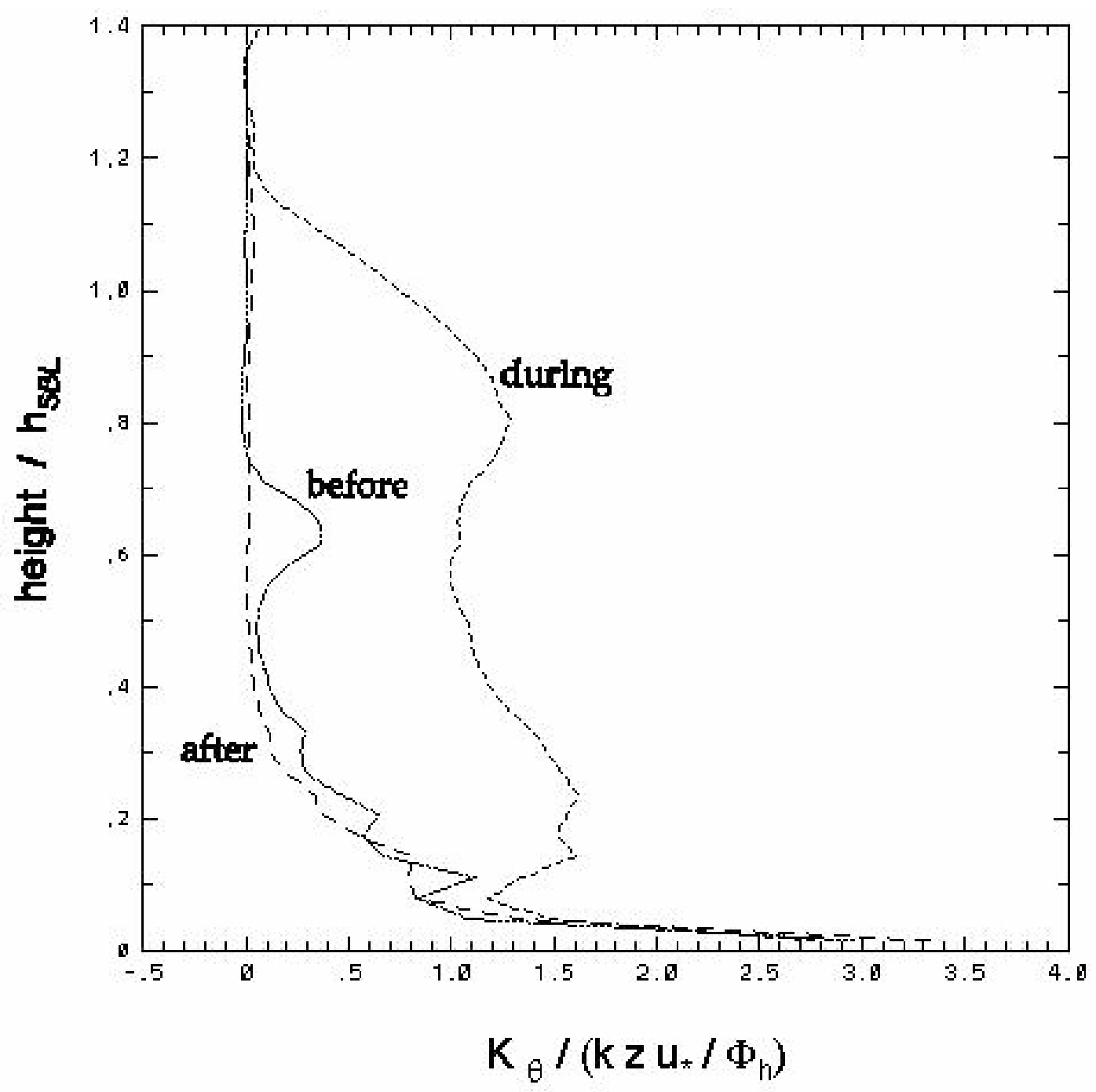

Figure 8.8: Normalized eddy diffusivity from Equation 8.2 for 10-minute periods before, during, and after episode described in Section 6.4 ; in the normalizing eddy diffusivity, $u_{*}=0.19 \mathrm{~m} / \mathrm{s}, \phi_{h}=1+5 z / L$, and $L=26.3 \mathrm{~m}$; height is normalized by SBL depth $h$. 


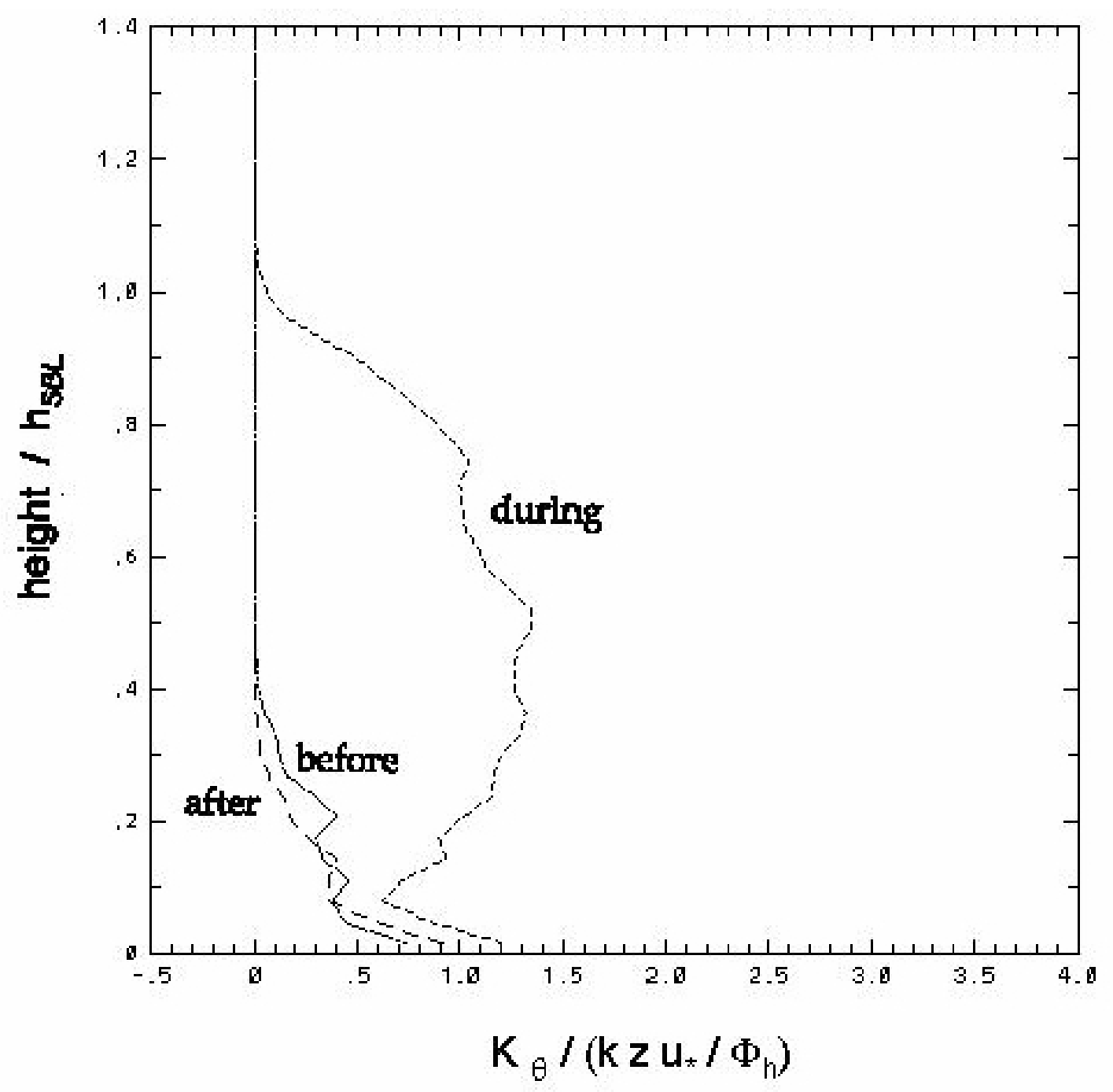

Figure 8.9: Normalized eddy diffusivity from Equation 8.3 for 10-minute periods before, during, and after episode described in Section 6.4 ; in the normalizing eddy diffusivity, $u_{*}=0.19 \mathrm{~m} / \mathrm{s}, \phi_{h}=1+5 z / L$, and $L=26.3 \mathrm{~m}$; height is normalized by SBL depth $h$. 


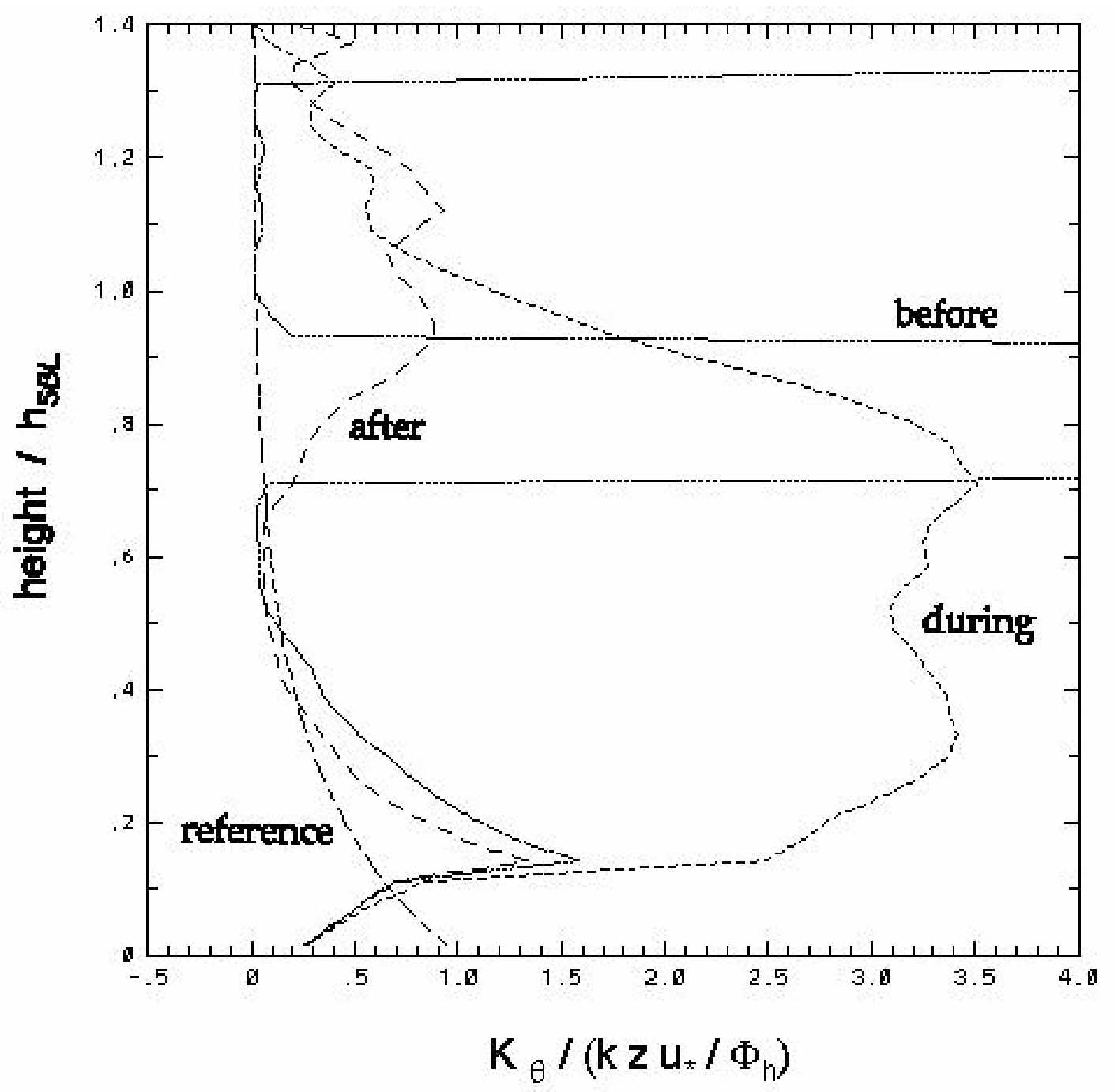

Figure 8.10: Normalized eddy diffusivity from Equation 8.4 modified for local scaling and using $\Lambda$ for z-less scaling, for 10-minute periods before, during, and after episode described in Section 6.4; the reference profile is from Equation 8.4 with the usual surface scaling; in the normalizing eddy diffusivity, $u_{*}=0.19 \mathrm{~m} / \mathrm{s}, \phi_{h}=1+5 z / L$, and $L=26.3 \mathrm{~m}$; height is normalized by SBL depth $h$. 


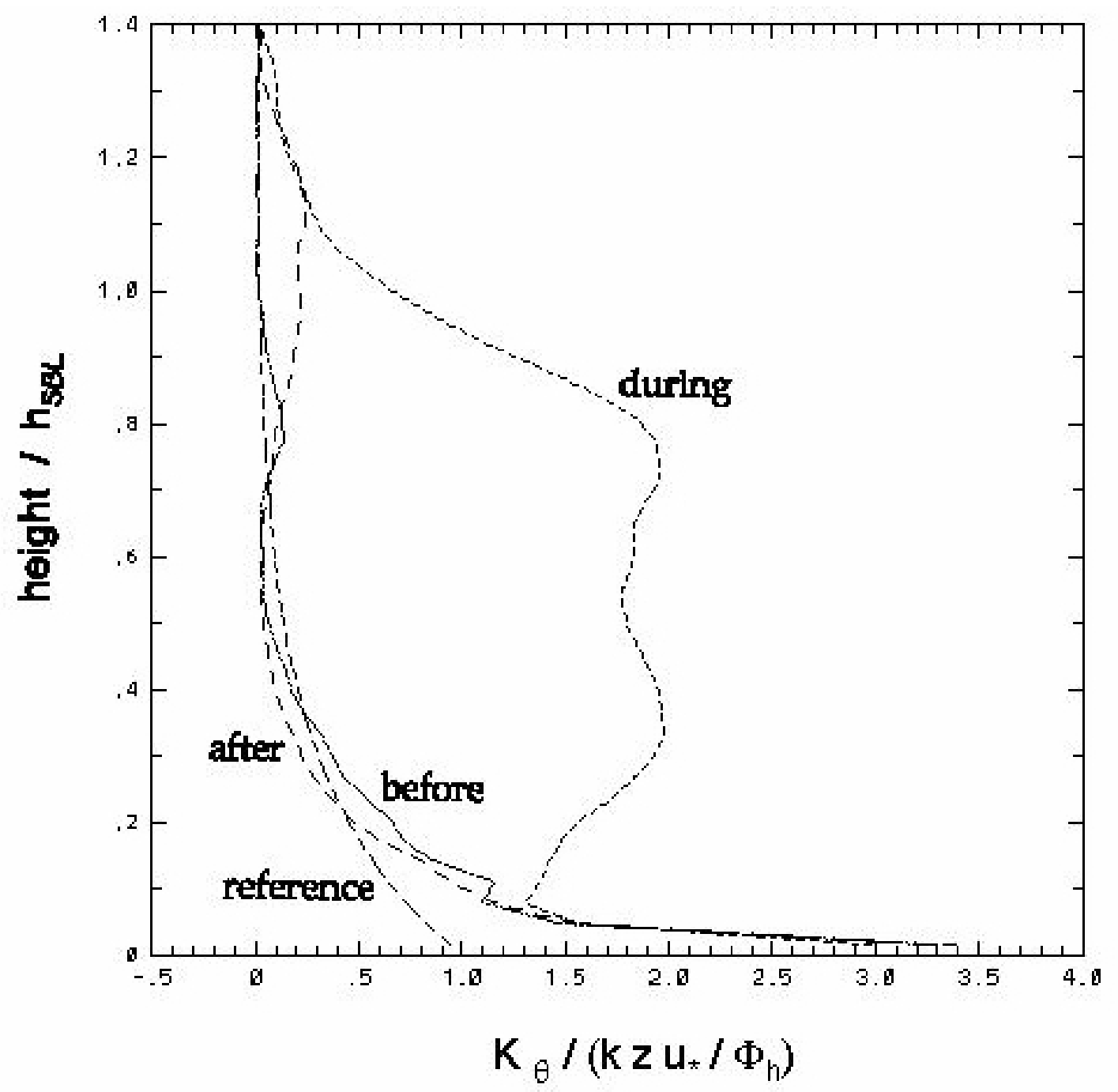

Figure 8.11: Normalized eddy diffusivity from Equation 8.4 modified for local scaling and using $u_{*}^{\text {local }} / N$ for z-less scaling, for 10-minute periods before, during, and after episode described in Section 6.4; the reference profile is from Equation 8.4 with the usual surface scaling; in the normalizing eddy diffusivity, $u_{*}=0.19 \mathrm{~m} / \mathrm{s}, \phi_{h}=1+5 z / L$, and $L=26.3$ $\mathrm{m}$; height is normalized by SBL depth $h$. 


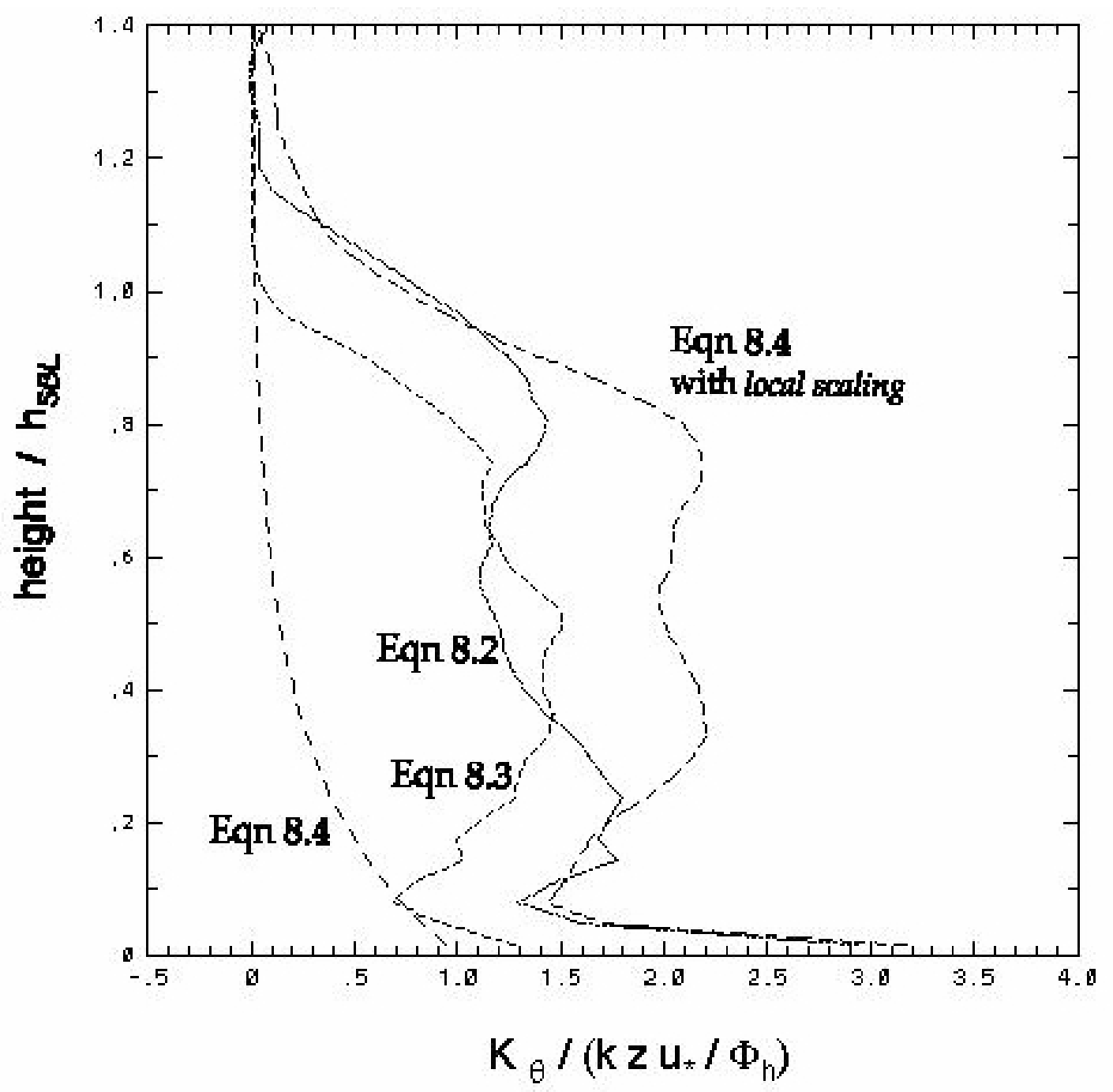

Figure 8.12: Normalized eddy diffusivity from Equations 8.2, 8.3, 8.4, and 8.4 with local scaling, for the 10-minute period during the episode described in Section 6.4; in the normalizing eddy diffusivity, $u_{*}=0.19 \mathrm{~m} / \mathrm{s}, \phi_{h}=1+5 z / L$, and $L=26.3 \mathrm{~m}$; height is normalized by SBL depth $h$. 


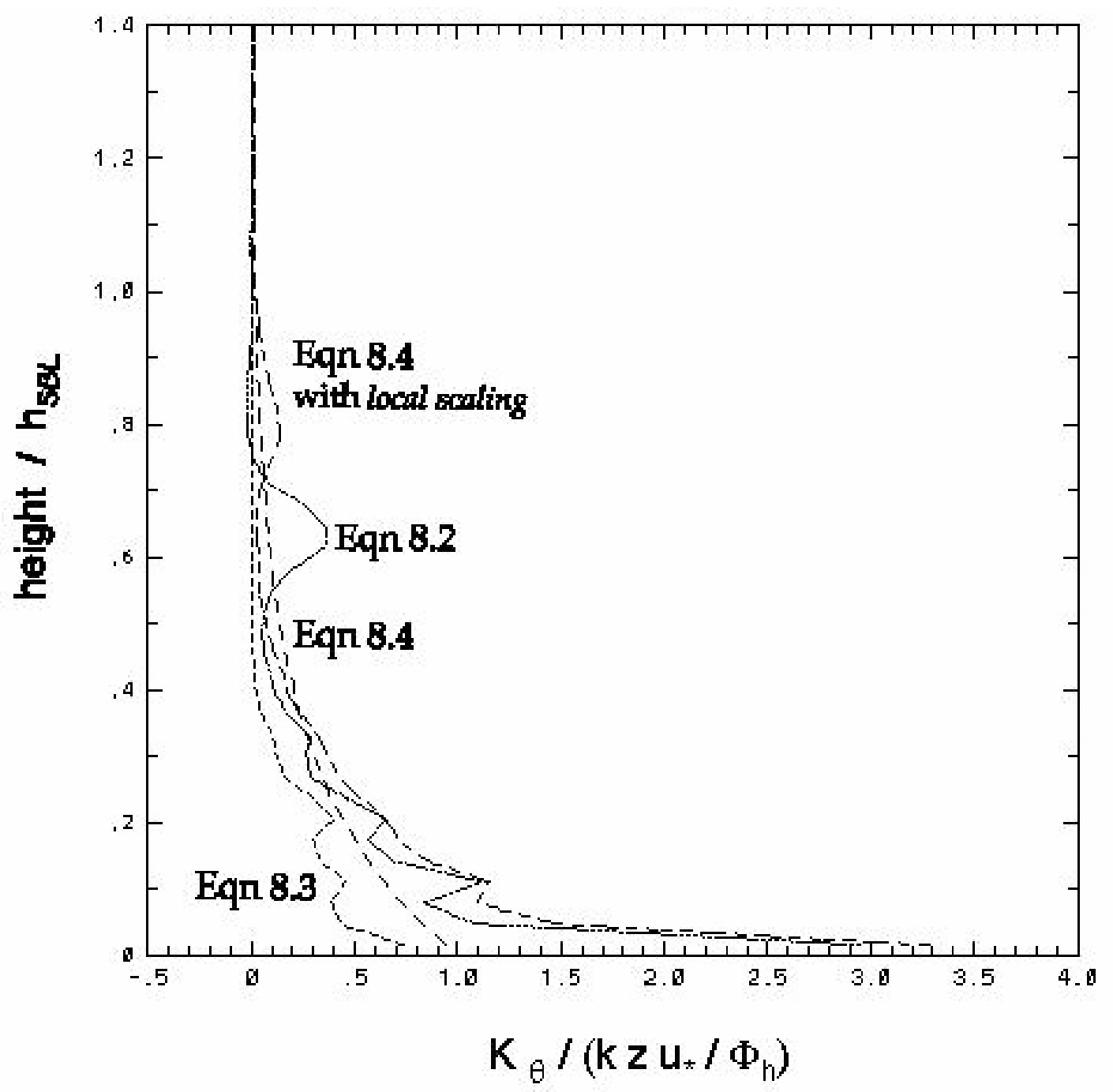

Figure 8.13: Normalized eddy diffusivity from Equations 8.2, 8.3, 8.4, and 8.4 with local scaling, for the 10-minute period before the episode described in Section 6.4 ; in the normalizing eddy diffusivity, $u_{*}=0.19 \mathrm{~m} / \mathrm{s}, \phi_{h}=1+5 z / L$, and $L=26.3 \mathrm{~m}$; height is normalized by SBL depth $h$. 


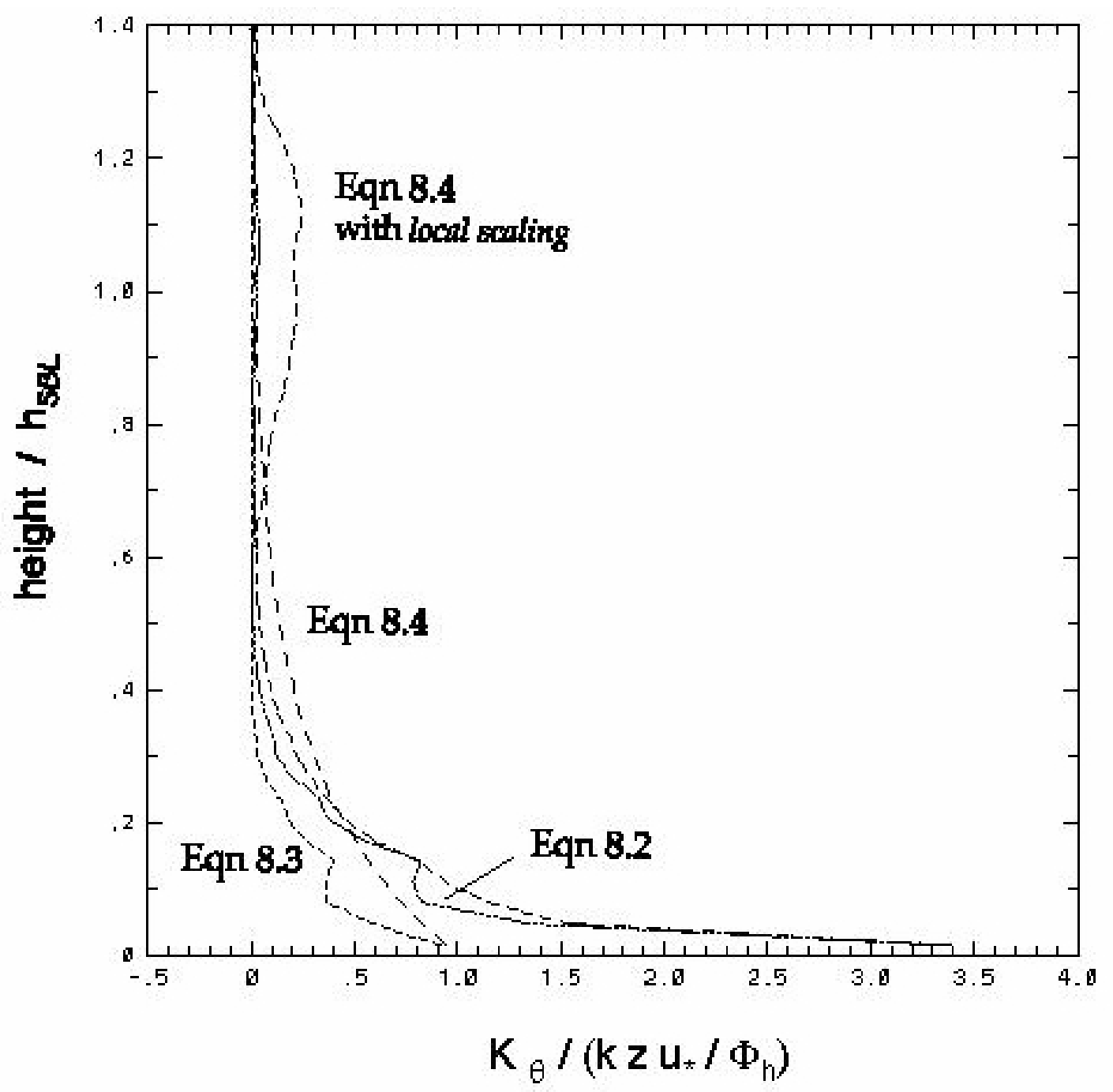

Figure 8.14: Normalized eddy diffusivity from Equations 8.2, 8.3, 8.4, and 8.4 with local scaling, for the 10-minute period after the episode described in Section 6.4; in the normalizing eddy diffusivity, $u_{*}=0.19 \mathrm{~m} / \mathrm{s}, \phi_{h}=1+5 z / L$, and $L=26.3 \mathrm{~m}$; height is normalized by SBL depth $h$. 


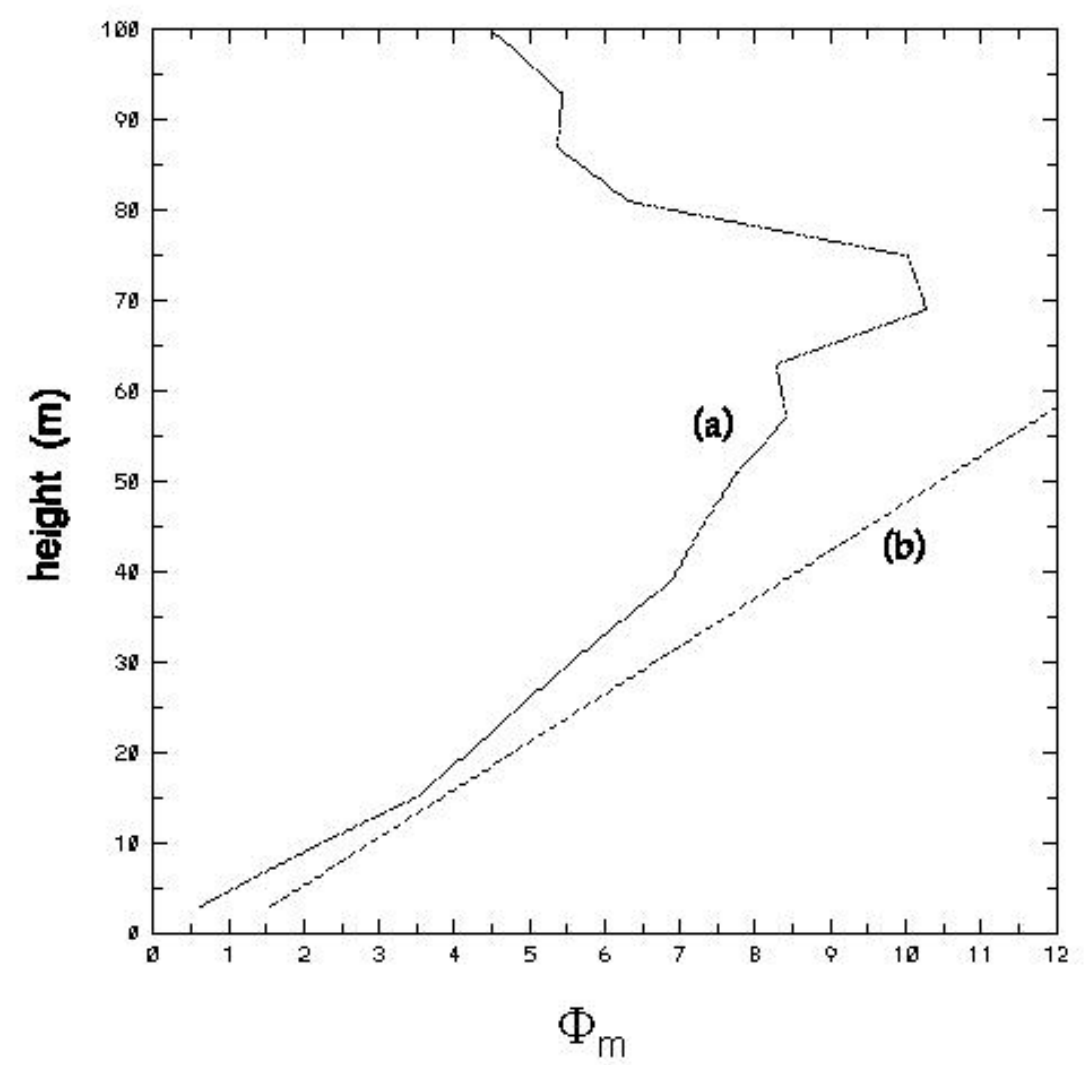

Figure 8.15: Estimate of nondimensional wind shear $\phi_{m}$ for SBL at hour 14 of Run $G 7.5 w t$ 02; solid line is from LES results, while dashed line is from Dyer relation $\phi_{m}=1+5 z / L$. 


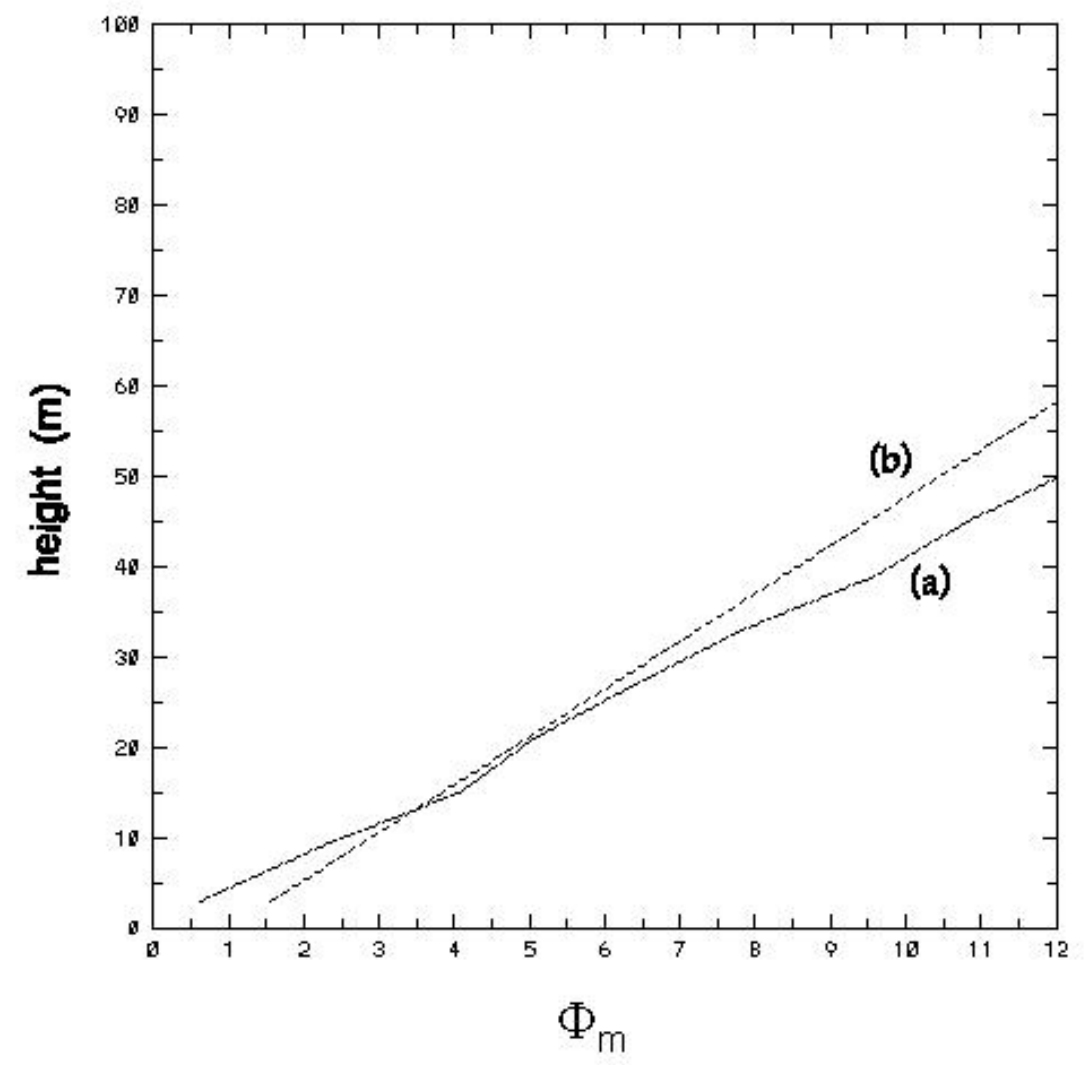

Figure 8.16: Estimate of nondimensional wind shear $\phi_{m}$ for SBL at hour 14 of Run G7.5wt-02; solid line is from LES results using local $u_{*}$, while dashed line is from Dyer relation $\phi_{m}=1+5 z / L$. 


\section{Chapter 9}

\section{Concluding Remarks}

This research has presented the results of using an LES approach that has integrated proven methods, rather than focusing on the development of a new SGS model. The application to the high Reynolds SBL has presented problems that have required special treatment in the numerics and details of the SGS model.

In the light of the results presented in the previous chapters, the following contributions have been made as a result of this research.

1. Use of a SGS turbulence model with backscatter provides an improved and more realistic simulation of the evolving SBL. In particular, periods of 'enhanced' turbulence are simulated that are often observed at night.

2. Results of the LES give a good characterization of the effects of stable stratification during SBL development. The mean velocity and temperature structure agrees with observations. A strong correlation between a 'stability forcing index' $\left(B_{0} / G^{2}|f|\right)$ and the bulk Richardson number $\left(R_{B}\right)$ is found, and is quantified as $R_{B}=8 / 3 F_{s}$.

3. Details of the turbulence structure (velocity variances, spectra) reveal particular effects of the damping on the vertical velocity fluctuations, which leads to increased anisotropy.

4. The advanced SGS turbulence model with backscatter supports investigations of the energetics in the evolving SBL that are unique. Kinetic energy transfer between scales can be evaluated. The important interaction of streamwise velocity and wall-normal shear stress underlying TKE backscatter that has been confirmed in DNS studies has been found in the present LES results as well.

5. Exchange between kinetic and potential energy in the SBL is not clearly evident in the mixing efficiency; although the mixing efficiency increases in the 
presence of increased wave-like activity during episodes of enhanced turbulence, this reflects a transient change that is more appropriately described as stirring.

6. Waves and turbulence are found to coexist in the simulated SBL. Although the exact mechanisms that lead to the onset of enhanced turbulence episodes has not been clearly quantified, our LES results suggest that the episodes are associated with the breakdown of large-scale wave-like activity in the upper part of the SBL. Phase angle analysis highlights the traditional downgradient turbulent flux near the surface during an episode, while in the middle of the SBL there is a mix of turbulence and internal waves. In the upper part of the SBL, countergradient turbulent flux is dominant and is thought to be caused by overturning events associated with wave-like activity.

7. For the first time, the upscale transfer of thermal energy has been quantified in an LES model. The 'backscatter' of thermal energy is found to be associated with coherent structures that cause ejections of cool, slow-moving air into the flow near the surface.

8. Coherent structures are abundant in the simulated SBL. Vortex cores identified in the SBL using the $\lambda_{2}$ method show a strong tendency to become aligned normal to the geostrophic forcing as the stratification increases near the surface.

9. LES-generated wind fields are used in simulations of dispersion in the SBL. The characteristics of the plumes of marker particles are markedly different in the CBL versus the SBL. Also, dispersion is increased during the periods of enhanced turbulence. The resulting differences in the volume of the simulated plume and the associated concentration patterns has implications for how best to conduct health assessment studies of hazardous materials released into the SBL. LES results are used to evaluate eddy diffusivities that are used in practical dispersion models. Practical estimates of eddy diffusivity represent eddy diffusion in the undisturbed SBL, but cannot capture effects of enhanced turbulence on eddy diffusion during episodes. 


\section{References}

Andre, J.C., 1983: On the variability of the nocturnal boundary-layer depth. Journal of the Atmospheric Sciences, 40, 2309-2311.

Andre, J.C., G. De Moor, P. Lacarrere, G. Therry, and R. du Vachat, 1978: Modeling the 24-hour evolution of the mean and turbulent structures of the planetary boundary layer. Journal of the Atmospheric Sciences, 35, 1861-1883.

Andren, A., 1995: The structure of stably stratified atmospheric boundary layers: A large-eddy simulation study. Quarterly Journal of the Royal Meteorological Society, 121, 961-985.

Andren, A., A. Brown, J. Graf, C.H. Moeng, P.J. Mason, F.T.M. Nieuwstadt, and U. Schumann, 1994: Large-eddy simulation of a neutrally stratified boundary layer: A comparison of four computer codes. Quarterly Journal of the Royal Meteorological Society, 120, 1457-1484.

Antonia, R.N., S. Rajagopalan, and AJ. Chambers, 1983: Conditional sampling of turbulence in the atmospheric surface layer. Journal of Climate and Applied Meteorology, 22, 69-78.

Appleby, J.F., and W.D. Ohmstede, 1964: Numerical Solution of the Distribution of Wind and Temperature in the Planetary Boundary Layer. USAERDAA-MET-5-64, U.S. Army Elect. Res. and Dev. Act., Ft. Huachuca, AZ.

Arakawa, A., 1966: Computational design for long-term numerical integration of the equations of fluid motion: Two dimensional incompressible Flow, Part I. Journal of Computational Physics, 1, 119-143.

Arya, S.P.S., 1975: Buoyancy effects in a horizontal flat-plate boundary layer. Journal of Fluid Mechanics, 68, 321-343.

Arya, S.P.S., 1981: Parameterizing the height of the stable atmospheric boundary layer, Journal of Applied Meteorology, 20, 1418-1428. 
Arya, S.P.S., and E.J. Plate, 1969: Modeling of the stably stratified atmospheric boundary layer. Journal of the Atmospheric Sciences, 26, 656665.

Asselin, R., 1972: Frequency filter for time integrations. Monthly Weather Review, 100, 487-490.

Bardina, J., J.H. Ferziger, and W.C. Reynolds, 1983: Improved Turbulence Models Based on Large-Eddy Simulation of Homogeneous, Incompressible Turbulent Flows. Report TF-19, Department of Mechanical Engineering, Stanford University, Stanford, CA, 174 pp.

Barnard, J.C., 2000: Intermittent Turbulence in the Very Stable Ekman Layer. PhD Dissertation, Department of Mechanical Engineering, University of Washington, Seattle, WA, 153pp.

Bernal, L.P., R.E. Breidenthal, G.L. Brown, J.H. Konrad, and A. Roshko, 1980: On the development of three-dimensional small scales in turbulent mixing layers. Turbulent Shear Flows 2, ed. LJ.S. Bradbury, F. Durst, B.E. Launder, F.W. Schmidt, J.H. Whitelaw, Springer-Verlag, Berlin, 305-313.

Blackadar, A.K, 1976: Modeling the nocturnal boundary layer. Third Symposium On Atmospheric Turbulence, Diffusion, and Air Quality, American Meteorological Society, Boston, 46-49.

Blackadar, A.K, 1979: High-resolution models of the planetary boundary layer. Advances in Environmental Science and Engineering, vol. 1, ed. J.R. Pfafflin and E.N. Zeigler, Gordon and Breach, New York, 50-85.

Bradshaw, P., 1980: Introduction to the Section on "Coherent Structures". Turbulent Shear Flows 2, ed. L.J.S. Bradbury, F. Durst, B.E. Launder, F.W. Schmidt, J.H. Whitelaw, Springer-Verlag, Berlin, 259-262.

Briggs, D.A., J.H. Ferziger, J.R. Koseff, and S.G. Monismith, 1998: Turbulent mixing in a shear-free stably stratified two-layer fluid. Journal of Fluid Mechanics, 354, 175-208.

Britter, R.E., J.C.R. Hunt, L. Marsh, and W.H. Snyder, 1983: The effects of stable stratification on turbulent diffusion and the decay of turbulence. Journal of Fluid Mechanics, 127, 27-44. 
Brost, R.A., and J.C. Wyngaard, 1978: A model study of the stably stratified planetary boundary layer. Journal of the Atmospheric Sciences, 35, 14271440 .

Brown, R.A., S.H. Derbyshire, and P.J. Mason: 1994: Large-eddy simulation of stable atmospheric boundary layers with a revised stochastic subgrid model. Quarterly Journal of the Royal Meteorological Society, 120, 14851512.

Brown, A.R., J.M Hobson, and N. Wood, 2001: Large-eddy simulation of neutral turbulent flow over rough sinusoidal ridges. Boundary-Layer Meteorology, 98, 411-441.

Businger, J.A., J.C. Wyngaard, Y. Izumi, and E.F. Bradley, 1971: Flux-profile relationships in the atmospheric surface layer. Journal of the Atmospheric Sciences, 28, 181-189.

Businger, J.A., and S.P.S. Arya, 1974: Height of the mixed layer in a stably stratified planetary boundary layer. Advances in Geophysics, 18A, 73-92.

Cantwell, B.J., 1981: Organized motion in turbulent flow. Annual Reviews in Fluid Mechanics, 13, 457-515.

Carati, D., S. Ghosal, and P. Moin, 1995: On the representation of backscatter in dynamic localization models. Physics of Fluids A, 7, 606616.

Caughey, S.J., J.C. Wyngaard, and J.C. Kaimal, 1979: Turbulence in the evolving stable boundary layer. Journal of the Atmospheric Sciences, 36, 1041-1052.

Caughey, S.J., 1982: Observed characteristics of the atmospheric boundary layer. Atmospheric Turbulence and Air Pollution Modeling, ed. F.T.M. Nieuwstadt and H. van Dop, D. Reidel Publ. Co., Dordrecht, 107-158.

Caughey, S.J., and S.G. Palmer, 1979: Some Aspects of Turbulence Structure through the Depth of the Convective Boundary Layer, Quarterly Journal of the Royal Meteorological Society, 105, 811-827. 
Cederwall, R.T., 1995: Large-eddy simulation of the development of stablystratified atmospheric boundary layers over cool flat surfaces. 11th Symposium on Boundary Layers and Turbulence, American Meteorological Society, Boston, 572-575.

Cederwall, R.T, and R.L. Street, 1997: Use of a dynamic subgrid-scale model for large-eddy simulation of the planetary boundary layer. 12th Symposium on Boundary Layers and Turbulence, American Meteorological Society, Boston, 215-216.

Cederwall, R.T, and R.L. Street, 1999: A study of turbulence in an evolving stable atmospheric boundary layer using large-eddy simulation. Turbulence and Shear Flow Phenomena - 1, ed. S. Banerjee, and J.K. Eaton, Begell House, Inc., New York, 583-588.

Cermak, J.E., and S.P.S. Arya, 1970: Problems of atmospheric shear flows and their laboratory simulation. Boundary-Layer Meteorology, 1, 40-60.

Chacin, J., and B. Cantwell, 1997: Study of Turbulence Structure Using the Invariants of the Velocity Gradient Tensor. Report No. TF-70, Stanford University, Stanford, CA, 130pp.

Chasnov, J.R., 1991: Simulation of the Kolmogorov inertial-subrange using an improved subgrid model. Physics of Fluids A, 3, 188-200.

Chollet, J.P., 1983: Two-point closure used for a sub-grid scale model in large-eddy simulation, Turbulent Shear Flows 4, ed. LJ.S. Bradbury, F. Durst, B. E. Launder, F.W. Schmidt, and J.H. Whitelaw, Springer-Verlag, Berlin, 62-72.

Clark R.A., J.H. Ferziger, and W.C. Reynolds, 1979: Evaluation of subgridscale models using an accurately simulated turbulent flow. Journal of Fluid Mechanics, 91, 1-16.

Clark, T.L, 1977: A small-scale dynamic model using a terrain-following coordinate transformation, Journal of Computational Physics, 24, 186-215.

Clark, T.L., and R.D. Farley, 1984: Severe downslope windstorm calculations in two and three spatial dimensions using anelastic interactive grid nesting: A possible mechanism for gustiness, Journal of the Atmospheric Sciences, 41, 329-350. 
Clarke, R.H., A.J. Dyer, R.R. Brook, D.G. Reid, and A.J. Troup, 1971: The Wangara Experiment: Boundary Layer Data, Technical Paper No. 19, CSIRO Div. of Meteorology and Physics, Melbourne, 340pp.

Coleman, G.N., J.H. Ferziger, and P.R. Spalart, 1992: Direct simulation of the stably stratified turbulent Ekman layer. Journal of Fluid Mechanics 244, 677-712.

Collis, S.S., 2001: Monitoring unresolved scales in multiscale turbulence modeling. Physics of Fluids, 13, 1800-1806.

Corrsin, S. 1974: Limitations of gradient transport models in random walks and in turbulence. Advances in Geophysics, 18A, 25-60.

Coulter, R.L., 1990: A case study of turbulence in the stable nocturnal boundary layer. Boundary-Layer Meteorology, 52, 75-91.

Deardorff, J.W., 1966: The counter-gradient heat flux in the lower atmosphere and in the laboratory. Journal of the Atmospheric Sciences, 23, 453-480.

Deardorff, J.W., 1970: A numerical study of three-dimensional turbulent channel flow at large Reynolds numbers. Journal of Fluid Mechanics 41, $677-712$

Deardorff, J.W., 1972a: Numerical investigation of neutral and unstable planetary boundary layers, Journal of the Atmospheric Sciences, 29, 91-115.

Deardorff, J.W., 1972b: Rate of growth of the nocturnal boundary layer. Proceedings of the Symposium on Air Pollution, Turbulence and Diffusion, ed. H.W. Church and R.E. Luna, Sandia, Albuquerque, 183-190.

Deardorff, J.W., 1973: Three-dimensional numerical modeling of the planetary boundary layer. Workshop on Micrometeorology, ed. D.N Haugen, American Meteorological Society, Boston, 271-311.

Deardorff, J.W., 1974a: Three-dimensional numerical study of the height and mean structure of a heated planetary boundary-layer. BoundaryLayer Meteorology, 7, 81-106.

Deardorff, J.W., 1974b: 'Three-dimensional numerical study of turbulence in an entraining mixed layer. Boundary-Layer Meteorology, 7, 199-226. 
Deardorff, J.W., 1976: Usefulness of liquid-water potential temperature in a shallow-cloud model. Journal of Applied Meteorology, 15, 98-102.

Deardorff, J.W., 1978: Efficient prediction of ground surface temperature and moisture, with inclusions of a layer of vegetation. Journal of Geophysical Research, 83, 1889-1903.

Deardorff, J.W., 1980: Stratocumulus-capped mixed layers derived from a three-dimensional model. Boundary-Layer Meteorology, 18, 495-527.

Deardorff, J.W., and G.E. Willis, 1985: Further results from a laboratory model of the convective planetary boundary layer. Boundary-Layer Meteorology, 32, 205-236.

De Bass, A F., and A G.M. Driedonks, 1985: Internal gravity waves in a stably stratified boundary layer. Boundary-Layer Meteorology, 31, 303-323.

Delage, Y., 1974: A numerical study of the nocturnal atmospheric boundary layer. Quarterly Journal of the Royal Meteorological Society, 100, 351-364.

Derbyshire, S.H., 1990: Nieuwstadt's stable boundary layer revisited. Quarterly Journal of the Royal Meteorological Society, 116, 127-158.

Dickey, T.D., and G.L. Mellor, 1980: Decaying turbulence in neutral and stratified fluids. Journal of Fluid Mechanics, 99, 13-31.

Ding, F., S.P. Arya, and Y.-L. Lin, 2001: Large-eddy simulations of the atmospheric boundary layer using a new subgrid-scale model. I. Slightly unstable and neutral cases. Environmental Fluid Mechanics, 1, 29-47.

Domaradski, J.A., and E.M. Saiki, 1997: A subgrid-scale model based on the estimation of unresolved scales of turbulence. Physics of Fluids, 9, 21482164 .

Domaradski, J.A., and K.-C. Loh, 1999: A subgrid-scale estimation model in the physical space representation. Physics of Fluids, 11, 2330-2342.

Dörnbrack, A., 1998: Turbulent mixing by breaking gravity waves. Journal of Fluid Mechanics, 375, 113-141. 
Dyer, A.J., 1974: A review of flux-profile relationships. Boundary-Layer Meteorology, 7, 363-372.

Ferziger, J.H., 1977: Large eddy numerical simulations of turbulent flows. AIAA Journal, 15, 1261-1267.

Ferziger, J.H., 1981: Higher-Level Simulations of Turbulent Flows, Report No. TF-16, Department of Mechanical Engineering, Stanford University, Stanford, CA.

Findikakis, A N. and R.L. Street, 1979: An algebraic model for subgrid-scale turbulence in stratified flows. Journal of the Atmospheric Sciences, 36, 1934-1949

Fletcher, C.A.J., 1991: Computational Techniques for Fluid Dynamics, Volume II. Springer-Verlag, Berlin, 493pp.

Fukui K, M. Nakajima, and H. Ueda, 1983: A Laboratory Experiment on Momentum and Heat Transfer in the Stratified Surface Layer, Quarterly Journal of the Royal Meteorological Society, 109, 661-676.

Garg, R.P., J.H. Ferziger, S.G. Monismith, and J.R. Koseff, 2000: Stably stratified turbulent channel flows. I. Stratification regimes and turbulence suppression mechanism. Physics of Fluids, 12, 2569-2594.

Germano, M., 1986: A proposal for a redefinition of turbulent stresses in the filtered Navier-Stokes equations. Physics of Fluids, 29, 2323-2324.

Germano, M., U. Piomelli, P. Moin, and W.H. Cabot, 1991: A dynamic subgrid-scale eddy viscosity model. Physics of Fluids A, 3, 1760-1765.

Geurts, B.J., 1997: Inverse modeling for large-eddy simulation. Physics of Fluids, 9, 3585-3587.

Gibson, C.H., 1981: Buoyancy effects in turbulent mixing: sampling turbulence in the stratified ocean. AIAA Journal, 19, 1394-1400.

Grotzbach, G., 1987: Direct numerical and large-eddy simulation of turbulent channel flows. Encyclopedia of Fluid Mechanics, 6, 1337-1391.

Hanna, S.R., G.A. Briggs, and R.P. Hosker, Jr., 1982: Handbook on Atmospheric Diffusion, DOE/TIC-11223, NTIS, Springfield, VA. 
Hansen, F.V., 1979: Engineering Estimates for the Calculation of Atmospheric Dispersion Coefficients, ASL Internal Rep., U.S. Army Atmos. Sci. Lab., White Sands Missile Range, NM, 19pp.

Hartel, C., and L. Kleiser, 1998: Analysis and modelling of subgrid-scale motions in near-wall turbulence. Journal of Fluid Mechanics, 356, 327-352.

Holt, S.E., J.R. Koseff, and J.H. Ferziger, 1992: A numerical study of the evolution and structure of homogeneous stably stratified sheared turbulence. Journal of Fluid Mechanics, 237, 499-539.

Holtslag, A.AM., and F.T.M. Nieuwstadt, 1986: Scaling the atmospheric boundary layer. Boundary-Layer Meteorology, 36, 201-209.

Hooke, W.H., F.F. Hall, Jr., and E.E. Gossard, 1973: Observed generation of an atmospheric gravity wave by shear instability in the mean flow of the planetary boundary layer. Boundary-Layer Meteorology, 5, 29-41.

Howell, J.F., and J. Sun, 1999: Surface-layer fluxes in stable conditions. Boundary-Layer Meteorology, 90, 495-520.

Hunt, J.C.R., 1985: Diffusion in the stably stratified atmospheric boundary layer. Journal of Climate and Applied Meteorology, 24, 1187-1195.

Hunt, J.C.R., J.C. Kaimal, and J.E. Gaynor, 1985: Some observations of turbulence structure in stable layers. Quarterly Journal of the Royal Meteorological Society, 111, 793-815.

Itsweire, E.C., K.N. Helland, and C.W. Van Atta, 1986: The evolution of grid-generated turbulence in a stable stratified fluid. Journal of Fluid Mechanics, 162, 299-338.

Itsweire, E.C., J.R. Koseff, D.A. Briggs, and J.H. Ferziger, 1993: Turbulence in stratified shear flows: implications for interpreting shear-induced mixing in the ocean. Journal of Physical Oceanography, 23, 1508-1522.

Jacobitz, F.G., S. Sarkar, and C.W. Van Atta, 1997: Direct numerical simulations of the turbulence evolution in a uniformly sheared and stably stratified flow. Journal of Fluid Mechanics, 342, 231-261. 
Jeong, J., and F. Hussain, 1995: On the identification of a vortex. Journal of Fluid Mechanics, 285, 69-94

Kaimal, J.C., J.C. Wyngaard, Y. Izumi, and O.R. Cote, 1972: Spectral characteristics of surface layer turbulence. Quarterly Journal of the Royal Meteorological Society 98, 563-589.

Kaimal, J.C., J.C. Wyngaard, D. Haugen, O.R. Cote, Y. Izumi, S.J. Caughey, and CJ. Readings, 1976: Turbulence in the convective boundary layer. Journal of the Atmospheric Sciences, 33, 2152-2169.

Kaltenbach, H-J, T. Gerz, and U. Schumann, 1994: Large-eddy simulation of homogeneous turbulence and diffusion in stably stratified shear flow. Journal of Fluid Mechanics, 280, 1-40.

Kau, C.J., and R.L. Peskin, 1972: Numerical Simulation of Turbulence and Diffusion in Three-Dimensional Flow. Technical Report No. 101, Geophysical Fluid Dynamics Program, Rutgers, New Brunswick, NJ.

Katapodes, F.V., R.L. Street, and J.H. Ferziger, 2000: Subfilter-scale scalar transport for large-eddy simulation. $14^{\text {th }}$ Symposium on Boundary Layers and Turbulence, American Meteorological Society, Boston, 474-475.

Kemp, J.R., and D.J. Thomson, 1996: Dispersion in stable boundary layers using large-eddy simulation. Atmospheric Environment, 30, 2911-2933.

Khalsa, S.J.S., 1980: Surface-layer intermittency investigated with conditional sampling, Boundary-Layer Meteorology, 19, 135-153.

Klemp, J.B., and D.K. Lilly, 1978: Numerical simulation of hydrostatic mountain waves. Journal of the Atmospheric Sciences, 35, 78-107.

Klemp, J.B., and R.B. Wilhelmson, 1978: The simulation of threedimensional convective storm dynamics. Journal of the Atmospheric Sciences, 35, 1070-1096.

Klemp, J.B., and D.R. Durran, 1983: An upper boundary condition permitting internal gravity wave radiation in numerical mesoscale models. Monthly Weather Review, 111, 430-444. 
Knight, D.D. and B.T. Murray, 1980: Theoretical investigation of interaction and coalescence of large structures in the turbulent mixing layer. Lecture Notes in Physics 136, ed. J. Jimenez, Springer-Verlag, Berlin, 62-92.

Komori, S., H. Ueda, F. Ogino, and T. Mizushina, 1983: Turbulence structure in stably stratified open-channel flow, Journal of Fluid Mechanics, 130, 1326.

Koop, C.G., and F.K. Browand, 1979: Instability and turbulence in a stratified fluid with shear. Journal of Fluid Mechanics, 93, 135-159.

Kosovic, B., 1997: Subgrid-scale modelling for the large-eddy simulation of high-Reynolds-number boundary layers. Journal of Fluid Mechanics, 336, 151-182.

Kosovic, B., and J.A. Curry, 2000: A large eddy simulation study of a quasisteady, stably stratified atmospheric boundary layer. Journal of the Atmospheric Sciences, 57, 1052-1068.

Kovasznay, LS.G., 1978: Large-Scale Structure in Turbulence: a Question or an Answer?, Structure and Mechanisms of Turbulence I, ed. H. Fiedler, Springer-Verlag, Berlin, 1-18.

Kovasznay, LS.G., V. Kibbons, and R. Blackwelder, 1970: Large-scale motion in the intermittent region of a turbulent boundary layer, Journal of Fluid Mechanics 41, 283-325.

Lamb, R.G., 1978: A numerical simulation of dispersion from an elevated point source in the convective boundary layer, Atmospheric Environment, 12, 1297-1304.

Lange, R., 1989: Tranferability of a three-dimensional air quality between two different sites in complex terrain. Journal of Applied Meteorology, 28, 665-679.

Laufer, J., 1975: New trends in experimental turbulence research. Annual Reviews in Fluid Mechanics, 7, 307-326.

Leith, C.E., 1990: Stochastic backscatter in a subgrid-scale model: plane shear mixing layer. Physics of Fluids A, 2, 297-299. 
Leonard, A., 1974: Energy cascade in large eddy simulations of turbulent fluid flows. Advances in Geophysics, 18, 237-248.

Lesieur, M., 1987: Turbulence in Fluids, Marinus Nijhoff Publishers, Dordrecht, The Netherlands, 286pp.

Lesieur, M., and O. Metais, 1996: New trends in large-eddy simulations of turbulence. Annual Reviews in Fluid Mechanics, 28, 45-82.

Leslie, D.C., and G.L. Quarini, 1979: The application of turbulence theory to the formulation of subgrid modelling procedures. Journal of Fluid Mechanics, 91, 65-91.

Lilly, D.K, 1965: On the computational stability of numerical solutions of time-dependent non-linear geophysical fluid dynamics problems. Monthly Weather Review, 93,11-26.

Lilly, D.K., 1967: The representation of small-scale turbulence in numerical simulation experiments. Proceedings of the IBM Scientific Computing Symposium on Environmental Sciences, IBM Form 32-1951, Yorktown Heights NY, 195-201.

Lilly, D.K, 1983: Stratified turbulence and the mesoscale variability of the atmosphere, Journal of the Atmospheric Sciences, 40, 749-761.

Lilly, D.K., 1992: A proposed modification of the Germano subgrid-scale closure method. Physics of Fluids A, 4, 633-635.

Loh, K.-C., and J.A. Domaradski, 199: The subgrid-scale estimation model on nonuniform grids. Physics of Fluids, 11, 3786-3792.

Lorenz, E.N., 1955: Available potential energy and the maintenance of the general circulation. Tellus, 7, 157-167.

Louis, J-F, 1979: A parameteric model of vertical eddy fluxes in the atmosphere. Boundary-Layer Meteorology, 17, 187-202.

Ludwig, F.L., and R.L. Street, 1995: Modification of multiresolution feature analysis for application to three-dimensional atmospheric wind fields. Journal of the Atmospheric Sciences, 52, 139-157. 
Lumley, J.L, 1979: Simulating turbulent transport in urban air-pollution models, Advances in Environmental Science and Engineering, vol. 1, ed. J.R. Pfafflin and E.N. Ziegler, Gordon and Breach, New York, 103-127.

Lumley, J.L., and H.A. Panofsky, 1964: The Structure of Atmospheric Turbulence. Interscience Publishers (Wiley), New York, 239pp.

Lund, T.S., 1991: On dynamic models for large eddy simulation. Annual Research Briefs - 1991, Center for Turbulence Research, NASA Ames Research Center / Stanford, 177-190.

Lund, T.S., and E.A. Novikov, 1993: Parameterization of subgrid-scale stress by the velocity gradient tensor. Annual Research Briefs - 1992, Center for Turbulence Research, NASA Ames Research Center / Stanford, 27-43.

MacCormack, R.W., and Baldwin, B.S., 1975: A numerical method for solving the Navier-Stokes equations with application to shock-boundary layer interactions. AIAA Paper 75-1.

Mahrt, L, and R.C. Heald, 1979: Comment on "Determining Height of the Nocturnal Boundary Layer, Journal of Applied Meteorology, 18, 383.

Manabe, S., 1969: Climate and the ocean circulation, I. The atmospheric circulation and the hydrology of the earth's surface, Monthly Weather Review, 97, 739-805.

Mason, P.J., and N.S. Callen, 1986: On the magnitude of the subgrid-scale eddy coefficient in large-eddy simulations of turbulent channel flow. Journal of Fluid Mechanics 162, 439-462.

Mason, P.J., and DJ. Thomson, 1987: Large-eddy simulations of the neutralstaticstability planetary boundary layer. Quarterly Journal of the Royal Meteorological Society, 113, 413-443.

Mason, P.J., 1994: Large-eddy simulation: a critical review of the technique. Quarterly Journal of the Royal Meteorological Society, 120, 1-26.

Mason, P.J. and S.H. Derbyshire, 1990: Large-eddy simulation of the stablystratified atmospheric boundary layer Boundary-Layer Meteorology, 53, 117-162. 
Mason, P.J., and D.J. Thomson, 1992: Stochastic backscatter in large-eddy simulations of boundary layers. J, Fluid Mech. 242, 51-78.

McEwan, A.D., 1983: The kinematics of stratified mixing through internal wavebreaking. Journal of Fluid Mechanics, 128, 59-80.

McMillan, O.J., and J.H. Ferziger, 1979: Direct testing of subgrid-scale models, AIAA Journal, 14, 1340-1346.

Melgarejo, J.W. and J.W. Deardorff, 1974: Stability functions for the boundary-layer resistance based on observed boundary-layer heights. Journal of the Atmospheric Sciences, 31, 1324-1333.

Mellor, G.L. and T. Yamada, 1974: A hierarchy of turbulence closure models for planetary boundary layers, Journal of the Atmospheric Sciences, 31, 1791-1806.

Metcalf, J.I., 1975: Gravity waves in a low-level inversion, Journal of the Atmospheric Sciences, 32, 351-361.

Metcalf, J.I., and D. Atlas, 1973: Microscale ordered motions and atmospheric structure associated with thin echo layers in stably stratified zones, Boundary-Layer Meteorology, 4, 7-35.

Moeng, C.-H., 1984: A large-eddy-simulation model for the study of planetary boundary-layer turbulence, Journal of the Atmospheric Sciences, 41, 2052-2062.

Montgomery, R.D., 1974: An Experimental Study of Grid Turbulence in a Thermally-Stratified Flow, PhD Dissertation, Univ. of Michigan.

Nappo, C.J., and G. Chimonas, 1992: Wave exchange between the ground surface and a boundary-layer critical level. Journal of the Atmospheric Sciences, 49, 1075-1091.

Nieuwstadt, F.T.M., 1985: A model for the stationary, stable boundary layer. Turbulence and Diffusion in Stable Environments, Ed. J.C.R Hunt, Clarendon Press, Oxford, 149-179.

Nieuwstadt, F.T.M., and R.A. Brost, 1986: The decay of convective turbulence. Journal of the Atmospheric Sciences, 43, 532-546. 
Nieuwstadt, F.T.M., P.J. Mason, C.-H. Moeng, and U. Schumann, 1991: Large-eddy simulation of the convective boundary layer: a comparison of four computer codes. Eighth Symposium. on Turbulent Shear Flows, Munich.

Nieuwstadt, F.T.M., 1981: The steady-state height and resistance laws of the nocturnal boundary layer: Theory compared with Cabauw observations. Boundary-Layer Meteorology, 20, 3-17.

Nieuwstadt, F.T.M.,1984a: Some aspects of the turbulent stable boundary layer. Boundary-Layer Meteorology, 30, 31-55.

Nieuwstadt, F.T.M.,1984b: The turbulent structure of the stable, nocturnal boundary layer, Journal of the Atmospheric Sciences, 41, 2202-2216.

Nieuwstadt, F.T.M., and A.G.M. Driedonks, 1979: The nocturnal boundary layer: A case study compared with model calculations. Journal of Applied Meteorology, 18, 1397-1405.

Nieuwstadt, F.T.M., and H. Tennekes, 1981: A rate equation for the nocturnal boundary-layer height, Journal of the Atmospheric Sciences, 38, 1418-1428.

Ogawa, Y., P.G. Diosey, K Uehara, and H. Ueda, 1985: Wind tunnel observations of flow and diffusion under stable stratification. Atmospheric Environment, 19, 65-74.

Ohmstede, W.D., 1985: Contributions to Large-Eddy Simulation: App. A, UCRL15755, Lawrence Livermore National Laboratory, Livermore, CA.

Orszag, S.A., 1971: Numerical simulation of incompressible flows within simple boundaries: Accuracy, Journal of Fluid Mechanics, 49, 75-112.

Osborn, T.R., 1980: Estimates of the local rate of vertical diffusion from dissipation measurements. Journal of Physical Oceanography, 10, 83-89.

Panofsky, H.A., and J.A. Dutton, 1984: Atmospheric Turbulence. WileyInterscience, New York, 397pp.

Pao, Y-H, 1973: Measurements of internal waves and turbulence in twodimensional stratified shear flows, Boundary-Layer Meteorology, 5, 177193. 
Piacsek, S.K., and G.P. Williams, 1970: Conservation properties of convection difference schemes. Journal of Computational Physics, 6, 392405.

Piccirillo, P.S., and C.W. Van Atta, 1997: The evolution of a uniformly sheared thermally stratified turbulent flow. Journal of Fluid Mechanics, 334, 61-86.

Pielke, R.A., 1984: Mesoscale Meteorological Modeling. Academic Press, Orlando, FL, 612pp.

Piomelli, U., 1999: Large-eddy simulation: achievements and challenges. Progress in Aerospace Science, 35, 335-362.

Piomelli, U., J.H. Ferziger, and P. Moin, 1987: Models for Large Eddy Simulation of Turbulent Channel Flows including Transpiration, Rpt. TF-32, Dept. Mech. Eng., Stanford Univ., Stanford, CA, 185pp.

Piomelli, U., J.H. Ferziger, P. Moin, and J. Kim, 1989: New approximate boundary conditions for large-eddy simulation of wall bounded flows. Physics of Fluids A, 1, 1061-1068.

Piomelli, U., T.A. Zang, C.G. Speziale, and M.Y. Hussaini, 1990: On the large-eddy simulation of transitional wall-bounded flows. Physics of Fluids A, 2, 257-265.

Piomelli, U., W.H. Cabot, P. Moin, and S. Lee, 1991: Subgrid-scale backscatter in turbulent and transitional flows. Physics of Fluids $A, 3$, 1766-1771.

Porte-Agel, , F., C. Meneveau, and M.B. Parlange, 1998: Some basic properties of the surrogate subgrid-scale heat flux in the atmospheric boundary layer. Boundary-Layer Meteorology, 88, 425-444.

Rees, J.M., and J.W. Rottman, 1994: Analysis of solitary disturbances over an Antarctic Ice Shelf. Boundary-Layer Meteorology, 69, 285-310.

Rees, J.M., W.J. Staszewski, W.J., and J.R. Winkler, 2001: Case study of a wave event in the stable atmospheric boundary layer overlying an Antarctic Ice Shelf using the orthogonal wavelet transform. Dynamics and Atmospheres and Oceans, 34, 245-261. 
Rehmann, C.R., 1995: Effects of Stratification and Molecular Diffusivity on the Mixing Efficiency of Decaying Grid Turbulence. Ph.D. thesis, Stanford University, Stanford, CA.

Rehmann, C.R., and J.R. Koseff, 2002: Mean potential energy change in stratified grid turbulence. Journal of Fluid Mechanics, submitted.

Reynolds, W.C., 1976: Computation of turbulent flows. Annual Reviews in Fluid Mechanics, 8, 183-208.

Riley, J.J., R.W. Metcalfe, and M.A. Weissman, 1981: Direct numerical simulations of homogeneous turbulence in density-stratified fluids. Nonlinear Properties of Internal Waves, ed. B.J. West, AIP Conference Proceedings No. 76, New York, 79-112.

Robert, A.J., 1966: The integration of a low order spectral form of the primitive meteorological equations. Journal of the Meteorological Society of Japan, 44, 237-244.

Rodi W., 1980: Turbulence Models and Their Application in Hydraulics, IAHR, Delft, The Netherlands, 5-46.

Rogallo, R.S., and P. Moin, 1984: Numerical simulation of turbulent flows. Annual Reviews in Fluid Mechanics, 16, 99-137.

Rohr, J.J., E.C. Itsweire, and C.W. Van Atta, 1984: Mixing efficiency in stably-stratified decaying turbulence. Geophysical and Astrophysical Fluid Dynamics, 29, 221-236.

Rohr, J.J., KN. Helland, E.C. Itsweire, and C.W. Van Atta, 1987: Turbulence in a stably stratified shear flow: a Progress Report. Turbulent Shear Flows 5, ed. F. Durst, B.E. Launder, F.W. Schmidt and J.H. Whitelaw, SpringerVerlag, Berlin, 67-76.

Rohr, J.J., E.C. Itsweire, KN. Helland, and C.W. Van Atta, 1988: Growth and decay of turbulence in a stably stratified shear flow. Journal of Fluid Mechanics, 195, 77-111.

Roshko, A, 1976: Structure of turbulent shear flows: a New Look. AIAA Journal, 14, 1349-1357. 
Saffman, P.G., 1978: Problems and progress in the theory of turbulence. Structure and Mechanisms of Turbulence II, ed. H. Fiedler, SpringerVerlag, Berlin, 273-306.

Sagaut, P., 2001: Large-Eddy Simulations for Incompressible Flows. Springer, Berlin, 319pp.

Saiki, E.M., C.-H. Moeng, and P.P. Sullivan, 2000: Large-eddy simulation of the stably stratified planetary boundary layer. Boundary-Layer Meteorology, 95, 1-30.

Salvetti, M.V., and S. Banerjee, 1995: A priori tests of a new dynamic subgridscale model for finite-difference large-eddy simulations. Physics of Fluids, 7, 2831-2847.

Sarghini, F., Piomelli, U., and Balars, E., 1999: Scale-similar models for large-eddy simulations. Physics of Fluids, 11, 1596-1607.

Schemm, C.E., and F.B. Lipps, 1976: Some results from a simplified threedimensional numerical model of atmospheric turbulence. Journal of the Atmospheric Sciences, 33, 1021-1041.

Schubert, J.F., 1977: Acoustic detection of momentum transfer during the abrupt transition from a laminar to a turbulent atmospheric boundary layer. Journal of Applied Meteorology, 16, 1292-1297.

Schumann, U., 1975: Subgrid-scale model for finite difference simulations of turbulent flows in plane channels and annuli. Journal of Computational Physics, 18, 376-404.

Schumann, U., 1994: Stochastic backscatter of turbulence energy and scalar variance from random subgrid-scale fluxes. Proceedings of the Royal Society of London, Series A (Mathematical and Physical Sciences), 451, 293318.

Schumann, U., G. Grotzbach, and L. Kleiser, 1980: Direct numerical simulation of turbulence. Prediction Methods for Turbulent Flows, ed. W. Kollmann, von Karman Inst. for Fluid Dynamics, Hemisphere Publishing Corporation, New York 124-258. 
Shah, K.B., and Ferziger, J.H., 1995: A new non-eddy viscosity subgridscale model and its application to channel flow. Annual Research Briefs: Center for Turbulence Research, NASA Ames Research Center and Stanford University.

Shih, L.H., J.R. Koseff, J.H. Ferziger, and C.R. Rehmann, 2000: Scaling and parameterization of stratified homogeneous turbulent shear flow. Journal of Fluid Mechanics, 412, 1-20.

Shir, C.C., and R.D. Bornstein, 1977: Eddy exchange coefficients in numerical models of the planetary boundary layer. Boundary-Layer Meteorology, 11, 171-185.

Smagorinsky, J., 1963: General circulation experiments with the primitive equations. I. The basic experiment. Monthly Weather Review, 91. 99-164.

Sommeria, G., 1976: Three-dimensional simulation of turbulent processes in an undisturbed trade wind boundary layer. Journal of the Atmospheric Sciences, 33, 216-241.

Spalart, P.R., 1989: Theoretical and numerical study of a three-dimensional turbulent boundary layer. Journal of Fluid Mechanics, 205, 319-340.

Speziale, C.G., 1985: Galilean invariance of subgrid scale stress models in the large eddy simulation of Turbulence. Journal of Fluid Mechanics, 156, 55-62.

Stillinger, D.C., KN. Helland, and C.W. Van Atta, 1983: Experiments on the transition of homogeneous turbulence to internal waves in a stratified fluid. Journal of Fluid Mechanics 131, 91-122.

Stolz, S., and N.A. Adams, 1999: An approximate deconvolution procedure for large-eddy simulation. Physics of Fluids, 11, 1699-1701.

Stull, R.B., 1983: A heat-flux history length scale for the nocturnal boundary layer. Tellus 35A, 219-230.

Stull, R.B., 1988: An Introduction to Boundary Layer Meteorology, Kluwer Academic Publishers, Dordecht, 11. 
Sullivan, P.P., McWilliams, J.C., and C-H Moeng, 1994: A subgrid-scale model for large-eddy simulation of planetary boundary-layer flows. Boundary-Layer Meteorology, 71, 247-276.

Taylor, J., 1992: The energetics of breaking waves in a resonantly forced internal wave field. Journal of Fluid Mechanics, 239, 309-340.

Taylor, G.I., and AK. Green, 1937: Mechanism of the production of small eddies from large ones. Proceedings of the Royal Society of London, A158, 499-521.

Tennekes, H. and J.L. Lumley, 1972: A First Course in Turbulence, MIT Press, Cambridge, MA, 300pp.

Thorpe, S.A., 1981: An experimental study of critical layers. Journal of Fluid Mechanics, 103, 321-344.

Tseng, Y.-H, and J.H. Ferziger, 2001: Mixing and available potential energy in stratified flows. Physics of Fluids, 13, 1281-1293.

Turner, J.,S., 1979: Buoyancy Effects in Fluids. Cambridge University Press, Cambridge, UK, 368pp.

Turner, J.S., 1981: Small-scale mixing processes. Evolution of Physical Oceanography. MIT Press, 236-262.

Van Atta, C.W., 1985: Stratified-turbulence experiments. Turbulence and Predictability in Geophysical Fluid Dynamics and Climate Dynamics, ed. M. Ghil, North-Holland Publ. Co., Amsterdam, 89-106.

Van Atta, C.W., KN. Helland, and E.C. Itsweire, 1984: The influence of stable stratification on spatially decaying vertically homogeneous turbulence. Turbulence and Chaotic Phenomena in Fluids, ed. T. Tatsumi, Elsevier, 519-528.

Venkatram, A., 1980: Estimating the Monin-Obukhov length in the stable boundary layer for dispersion calculations. Boundary-Layer Meteorology, 19, 481-485.

Wang, Q., and K.D. Squires, 1996: Large-eddy simulation of particle-laden turbulent channel flow. Physics of Fluids, 8, 1207-1223. 
Webster, C.A.G., 1964: An experimental study of turbulence in a densitystratified shear flow. Journal of Fluid Mechanics 19, 221-245.

Weinstock, J., 1984: Effect of gravity waves on turbulent decay in stratified fluids. Journal of Fluid Mechanics 140,11-26.

Willis, G.E., and J.W. Deardorff, 1974: A laboratory model of the unstable planetary boundary layer. Journal of the Atmospheric Sciences, 31, 12971307.

Wippermann, F., 1973: The Planetary Boundary Layer of the Atmosphere, Deutschen Wetterdienst, $346 \mathrm{pp}$.

Wong, V.C., 1992: A proposed statistical-dynamic closure method for the linear or nonlinear subgrid-scale stresses. Physics of Fluids A, 4, 10801082.

Wyngaard, J.C., 1975: Modeling the planetary boundary layer - extension to the stable case. Boundary-Layer Meteorology, 9, 441-460.

Wyngaard, J.C., ed., 1984: Large-Eddy Simulation: Guidelines for its Application to Planetary Boundary Layer Research, Contract No. 0804, U.S. Army Research Office, P.O. Box 12211, Research Triangle Park, NC 27709.

Wyngaard, J.C., and R.A. Brost, 1984: Top-down and bottom-up diffusion of a scalar in the convective boundary layer. Journal of the Atmospheric Sciences, 41, 102-112.

Yamada, T., and G. Mellor, 1975: A simulation of the Wangara atmospheric boundary layer data. Journal of the Atmospheric Sciences, 32, 2309-2329.

Yoshizawa, A., 1989: Subgrid-scale modeling with a variable length scale. Physics of Fluids A, 1, 1293-1295.

Zang, Y., R.L. Street, and J.R. Koseff, 1993: A dynamic mixed subgrid-scale model and its application to turbulent recirculating flows. Physics of Fluids A, 5, 3186-3196.

Zeman, O., 1979: Parameterization of the dynamics of stable boundary layers and nocturnal jets, Journal of the Atmospheric Sciences, 36, 792-804. 
Zeman, O., 1981: Progress in the modeling of planetary boundary layers. Annual Reviews of Fluid Mechanics, 13, 253-272.

Zeman, O., and J.L. Lumley, 1979: Buoyancy effects in entraining turbulent boundary layers: a second-order closure study. Turbulent Shear Flows 1, ed. F. Durst, B.E. Launder, F.W. Schmidt and J.H. Whitelaw, SpringerVerlag, Berlin, 295-306.

Zilitinkevich, S.S., 1972: On the determination of the height of the Ekman boundary layer. Boundary-Layer Meteorology, 3, 141-145. 




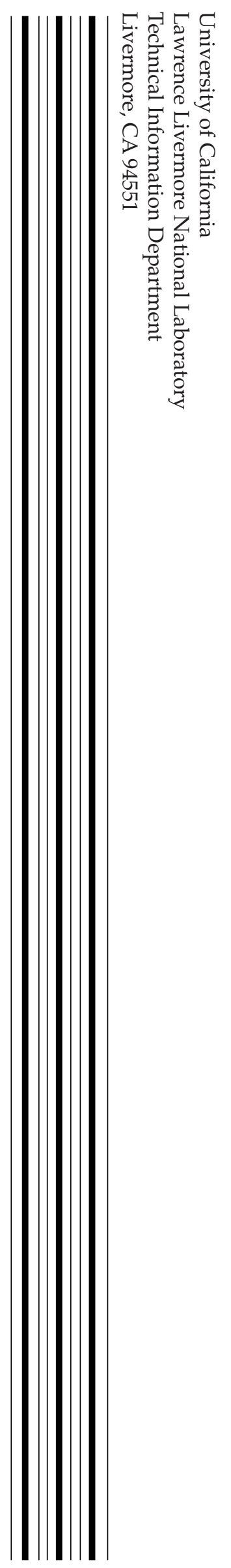

